\title{
Auswertung und Ergebnisse
}

Die Auswertung und Ergebnisse der vorliegenden Arbeit lassen sich in zehn Felder unterteilen. Im ersten Abschnitt 6.1 werden die Herkunft und die Soziodemographie der Professoren mit Migrationshintergrund in den Mittelpunkt gestellt. Im Anschluss werden die Bildungs- und Berufsverläufe (6.2) näher beschrieben. In Abschnitt 6.3 geht es um das Verhältnis von Forschung und Lehre sowie Funktionen und Drittmittel bei den Professoren. Daran anschließend werden das Berufsverständnis und die Beurteilung von Hochschulreformen (6.4) näher untersucht. In Abschnitt 6.5 werden die berufliche Zufriedenheit und die Arbeitsbedingungen analysiert, bevor es in Abschnitt 6.6 um die internationale Mobilität und internationale Aktivitäten von Professoren mit Migrationshintergrund geht. Zuwanderungsgeschichte, familiäre Situation und Zukunftspläne werden in Abschnitt 6.7 beschrieben und untersucht. Im Anschluss geht es in Abschnitt 6.8 um die Integration, die soziale Teilhabe und die Transnationalität der Professoren. Darauf aufbauend werden Fragen der Diskriminierung anhand von Vor- und Nachteilen sowie Erfahrungsbeispielen der Professoren (6.9) diskutiert. Im letzten Abschnitt 6.10 wird das Selbstverständnis der Professoren mit Migrationshintergrund in den Blick genommen.

\subsection{Herkunft und Demographie der Professoren mit Migrationshintergrund}

Im ersten Unterkapitel stehen Herkunft und Demographie der Professoren im Mittelpunkt. Die ersten sechs Teile beziehen sich auf migrationsspezifische Merkmale wie Geburtsland und Staatsangehörigkeit, Migrationstypen, Aufenthaltsdauer, die erweiterte Staatsangehörigkeit und die Muttersprache. Im fünften Teil wird untersucht, wie stark große Zuwanderungsgruppen und ihre Nachkommen wie 
Arbeitsmigranten, Flüchtlinge und (Spät-)Aussiedler repräsentiert sind und welche Bedeutung Zuwanderung aus dem europäischen Ausland zukommt. Die Teile sieben und acht beschreiben die Verteilung nach Geschlecht und sozialer Herkunft. In den letzten drei Teilen geht es um berufsspezifische Verteilungen nach Hochschulart, Besoldungsgruppe und Fächergruppe. Zugleich werden wichtige Zusammenhänge zwischen den verschiedenen Merkmalen herausgestellt.

Bildungsforschung über Menschen mit Migrationshintergrund differenziert häufig vor allem nach Herkunftsland bzw. Herkunftsregion. Wenngleich es intuitiv sicher interessant erscheint, zu untersuchen, inwieweit sich in Bildung und Beruf Unterschiede je nach Herkunftsland zeigen, birgt ein solcher Ansatz immer die Gefahr des methodologischen Nationalismus. Das heißt, dass jegliche Unterschiede exklusiv oder zumindest primär über das jeweilige Herkunftsland erklärt werden, ohne mögliche andere Einflussfaktoren systematisch zu berücksichtigen. Um diese Gefahr zu umgehen, werden in dieser Arbeit Unterschiede und Gemeinsamkeiten von Migranten anhand ausgewählter Merkmale untersucht, die sowohl die Migrationsbiographie als auch demographische und berufliche Aspekte umfassen. Hinsichtlich der Migrationsbiographie wird differenziert nach Zuwanderungsalter, Aufenthaltsdauer in Deutschland, Staatsangehörigkeit (deutsche, ausländische oder doppelte Staatsangehörigkeit), Herkunftsregion und Entwicklungsstand des Herkunftslandes. Bei den demographischen Merkmalen werden Geschlecht, Bildungsherkunft und Alter berücksichtigt. Hinsichtlich der beruflichen Merkmale werden schließlich Hochschulart, Besoldungs- und Fächergruppe näher in den Blick genommen.

\subsubsection{Geburtsland und Staatsangehörigkeit}

Die häufigsten Indikatoren zur Beschreibung der Herkunft stellen die Staatsangehörigkeit und das Geburtsland dar. Hinsichtlich der Aussagekraft beider Indikatoren zeigen sich allerdings gewisse Einschränkungen. Bei der Staatsangehörigkeit gestaltet sich einerseits eine eindeutige Zuordnung für Personen mit mehr als einer Staatsangehörigkeit schwierig. Andererseits können Staatsangehörigkeiten sowohl neu erworben als auch aufgegeben oder verloren werden. Der Wandel nationaler Gesetzgebungen hinsichtlich des Einbürgerungsrechts erschwert dabei sowohl historische Vergleiche einzelner nationaler Rechtsordnungen im Laufe der Zeit als auch internationale Vergleiche zwischen den Rechtssystemen verschiedener Staaten. Eine zweite Möglichkeit liegt darin, die Herkunft einer Person anhand ihres Geburtslandes zu beschreiben. Diese Form der Operationalisierung hat allerdings die Schwäche, dass beispielsweise Kinder von 
Eltern, die nur vorübergehend im Ausland gelebt haben, unter Umständen einem Herkunftsland zugeordnet werden, dem hinsichtlich der eigenen Sozialisation und Biographie nur ein geringer Stellenwert zukommt.

Bei der Befragung der Professoren mit Migrationshintergrund ergeben sich je nach Operationalisierung deutliche Unterschiede. Jeder fünfte Professor mit Migrationshintergrund ist in Deutschland geboren. Damit stellt Deutschland das häufigste Geburtsland dar vor Österreich (14\%), der Schweiz (9\%) und den USA (7 \%). Deutlich höher liegt der Anteil an Professoren mit deutscher Staatsangehörigkeit, wozu aktuell jeder zweite Professor mit Migrationshintergrund zählt. Dabei ist zu beachten, dass über ein Viertel der Professoren (26\%) zwei oder mehr Staatsangehörigkeiten hat. Ähnlich wie beim Geburtsland zeigen sich auch hier hohe Anteile bei den Professoren mit einer Staatsangehörigkeit aus Österreich (14\%), der Schweiz (12\%) und den USA (9\%). Ein Vergleich zwischen deutschen Professoren mit Migrationshintergrund und ausländischen Professoren nach Geburtsland zeigt, dass in etwa ein Drittel der Professoren mit deutscher Staatsangehörigkeit bereits in Deutschland geboren ist. Demgegenüber sind erwartungsgemäß fast alle ausländischen Professoren außerhalb von Deutschland geboren, wobei es hier eine kleine Gruppe gibt, die trotz Geburt in Deutschland keine deutsche Staatsangehörigkeit besitzt (vgl. Abbildung 6.1).

\subsubsection{Migrationstypen}

Gegenstand der Erhebungen im Rahmen der MOBIL-Studie war allerdings nicht nur das Geburtsland, sondern auch das Alter zum Zeitpunkt der Zuwanderung. Da es biographisch einen erheblichen Unterschied macht, ob Professoren bereits als Kleinkind oder erst anlässlich der Berufung zugewandert sind, wird im Folgenden eine Differenzierung nach dem Zuwanderungsalter vorgenommen. ${ }^{1}$

Um diese Heterogenität angemessen abbilden zu können, wurden vom Autor - basierend auf dem Zuwanderungsalter - drei Migrationstypen gebildet ${ }^{2}$ :

- Early Migrants: Zweite Generation ${ }^{3}$ sowie Professor/-innen, die als Kinder und Jugendliche (unter 18 Jahren) nach Deutschland zugewandert sind,

\footnotetext{
${ }^{1}$ Die nachfolgende Erläuterung war bereits Gegenstand der Veröffentlichung Engel (2017).

${ }^{2}$ Die Terminologien werden auch in der Studie»Changing Academic Profession « verwendet (vgl. Huang u. a. 2014). Im Abschlussbericht des MOBIL-Projektes (Neusel u. a. 2014) werden sechs Mobilitätstypen unterschieden, die auf der Grundlage des Zuwanderungsalters und des ersten Bildungsabschlusses in Deutschland entwickelt wurden.

${ }^{3}$ Zweite Generation bezieht sich auf Personen, die bereits in Deutschland geboren sind.
} 


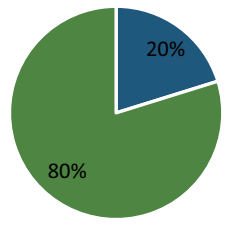

- Geburtsland Deutschland - Im Ausland geboren

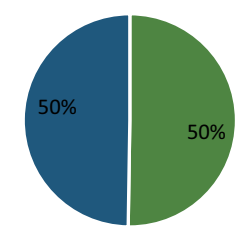

- Deutsche StA. - Ausländische StA.

Ausländische StA.

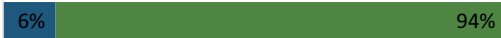

Deutsche StA

\section{$66 \%$}

- Geburtsland Deutschland Im Ausland geboren

Abbildung 6.1 Geburtsland und Staatsangehörigkeiten von Professoren mit Migrationshintergrund. (Quelle: MOBIL 2012/2013 ( $\mathrm{N}=203$ ) $\mathrm{p}<0,001$ p-value basiert auf $\chi^{2}-$ Test)

- Student Migrants: Professor/-innen, die zwischen ihrem 18. und 31. Lebensjahr nach Deutschland eingewandert sind,

- Professional Migrants: Professor/-innen, die bereits 32 Jahre oder älter waren, als sie nach Deutschland migriert sind.

Diese heuristische Einteilung wurde aus folgenden Gründen vorgenommen. Die Gruppe der Early Migrants weist das gemeinsame Merkmal auf, dass alle Personen dieser Gruppe einen Teil oder ihre gesamte „Schulsozialisation“ bereits in Deutschland durchlaufen haben. Zudem unterscheiden sich die zugewanderten Early Migrants von den Student Migrants dahingehend, dass sie zum Zeitpunkt der Zuwanderung noch nicht volljährig waren. Daher ist davon auszugehen, dass hier ein Großteil der Zuwanderungsprozesse weniger auf einer autonomen eigenen Entscheidung basierte, sondern stärker durch Entscheidungen der Eltern bedingt war. Die Bezeichnung Student Migrants bedeutet nicht zwangsläufig, dass die Professoren zum Studium nach Deutschland gekommen sind, sondern dass sie in einer Lebensphase nach Deutschland zugewandert sind, in die häufig das Hochschulstudium und die Promotion fallen. Beispielsweise war eine kleine Gruppe der Professoren mit Migrationshintergrund (4\%) jünger als 32 Jahre zum Zeitpunkt der Erstberufung. Die Entscheidung, Professoren als Student Migrants bis 
zu einem Zuwanderungsalter von 31 Jahren zu klassifizieren, begründet sich darin, dass die Professorenschaft in Deutschland Befragungen zufolge zum Zeitpunkt der Promotion durchschnittlich 31 Jahre alt ist (Schomburg et al. 2012, S. 27). Der Begriff Professional Migrants wurde gewählt, da diese Gruppe zum Zeitpunkt der Zuwanderung nach Deutschland in der Regel bereits alle berufsqualifizierenden Bildungsabschlüsse (bis hin zur Promotion) erworben hat.
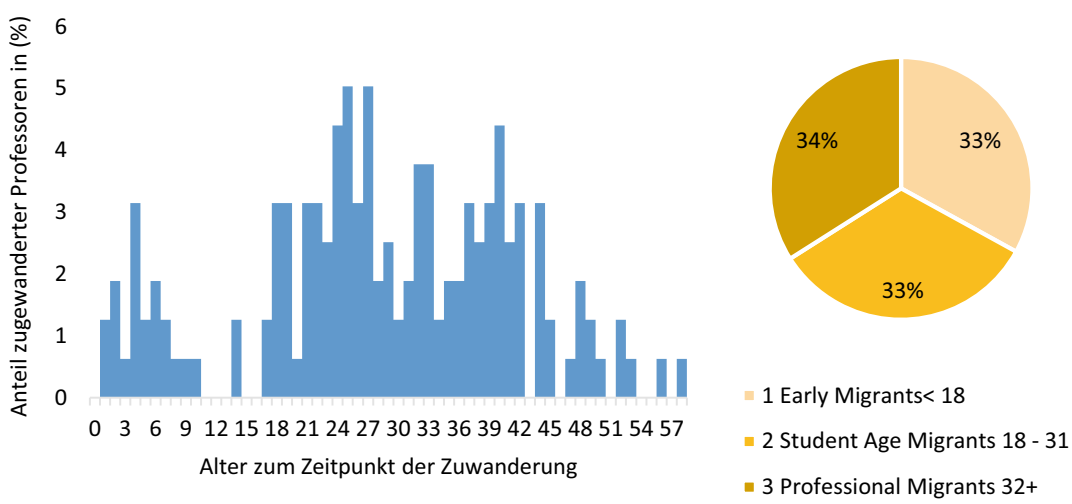

Abbildung 6.2 Alter zum Zeitpunkt der Zuwanderung und Migrationstypen. (Quelle: MOBIL 2012/2013 ( $\mathrm{N}=159)$ und $(\mathrm{N}=203)$ )

Nach dieser Einteilung lassen sich jeweils $33 \%$ als Early bzw. Student Migrants und $34 \%$ als Professional Migrants klassifizieren. Neben den $20 \%$ der Professoren, die bereits in Deutschland geboren wurden, sind weitere $13 \%$ als Kinder oder Jugendliche nach Deutschland gekommen, der Großteil von ihnen bereits in den ersten zehn Lebensjahren. Die größte Zuwanderung findet zwischen dem 20. und 30. Lebensjahr statt. Die Professional Migrants sind größtenteils vor dem 45. Lebensjahr nach Deutschland gekommen. Der deutlich geringere Anteil von Professoren, die zum Zeitpunkt der Zuwanderung bereits über 45 Jahre alt waren, lässt sich unter anderem damit erklären, dass ein Teil der Professoren das entsprechende Alter auch heute noch nicht erreicht hat. Zugleich deutet dieser Befund aber ebenfalls darauf hin, dass ab einem bestimmten Alter die Entscheidung, den Lebensmittelpunkt in ein anderes Land zu verlegen, deutlich seltener getroffen wird (vgl. Abbildung 6.2).

Das Durchschnittsalter liegt bei 48,8 Jahren. Early Migrants stellen 47,2 Jahren die jüngste Gruppe dar, vor den Professional Migrants mit 49,4 und den Student 
Migrants mit 49,7 Jahren. ${ }^{4}$ Insbesondere in der jüngeren Generation unter 45 Jahren stellen Early Migrants mit Abstand die größte Gruppe dar (41\%). ${ }^{5}$ Interessant für kommende Forschungsarbeiten wäre die Frage, inwieweit sich darin möglicherweise eine Entwicklung zeigt in Richtung einer Verbesserung der Teilhabechancen von Migranten, die als Kinder hier aufgewachsen sind und z. T. bereits in zweiter Generation hier leben.

\subsubsection{Alter und Aufenthaltsdauer in Deutschland}

Ein weiterer wichtiger Aspekt, um den Migrationsverlauf zu beschreiben, ist neben dem Zuwanderungsalter die Frage, wie lang Professoren bereits in Deutschland leben. So können beispielsweise Professional Migrants, die mit Anfang 30 nach Deutschland gekommen sind, erst seit wenigen Jahren hier leben, aber auch möglicherweise schon über 30 Jahre, wenn sie zum Zeitpunkt der Erhebung kurz vor der Pensionierung stehen. Um die Aufenthaltsdauer seit der Zuwanderung differenziert abzubilden, ist zunächst die generelle Altersstruktur der Professoren mit Migrationshintergrund darzulegen. Die Altersspanne der Professoren variiert dabei zwischen 31 und 67 Jahren. Das Durchschnittsalter liegt zum Zeitpunkt der Erhebung 2012/13 für Juniorprofessoren (W1) bei 37 Jahren, für W2-/C3-/C2-Professoren an Universitäten bei 49 Jahren, und sowohl bei Fachhochschulprofessoren als auch bei W3-/C4-Professoren an Universitäten bei 51 Jahren. Damit liegt das Durchschnittsalter der Professoren mit Migrationshintergrund unter dem Altersdurchschnitt in der gesamten Professorenschaft. Hier liegt das Durchschnittsalter laut der deutschlandweit durchgeführten CAP-Studie für Universitätsprofessoren bei 53 Jahren und für Fachhochschulprofessoren bei 52 Jahren (Teichler et al. 2017). Um ferner eine differenzierte Analyse nach Altersgruppen vornehmen zu können, wurde dieser Personenkreis in drei zahlenmäßig etwa gleich große Altersgruppen unterteilt. Während ein Drittel der Gruppe aus Professoren besteht, die jünger als 45 Jahre sind, bestehen die anderen beiden Drittel jeweils aus Professoren im Alter zwischen 45 und 53 Jahren bzw. von über 53 Jahren (vgl. Abbildung 6.3).

In etwa jeder vierte Professor mit Migrationshintergrund ist erst im Laufe der letzten zehn Jahre nach Deutschland gekommen. Zugleich leben aber über die

\footnotetext{
${ }^{4}$ Unterschiede sind statistisch nicht signifikant.

${ }^{5}$ Hier ist zu berücksichtigen, dass Professional Migrants möglicherweise erst später zuwandern.
} 


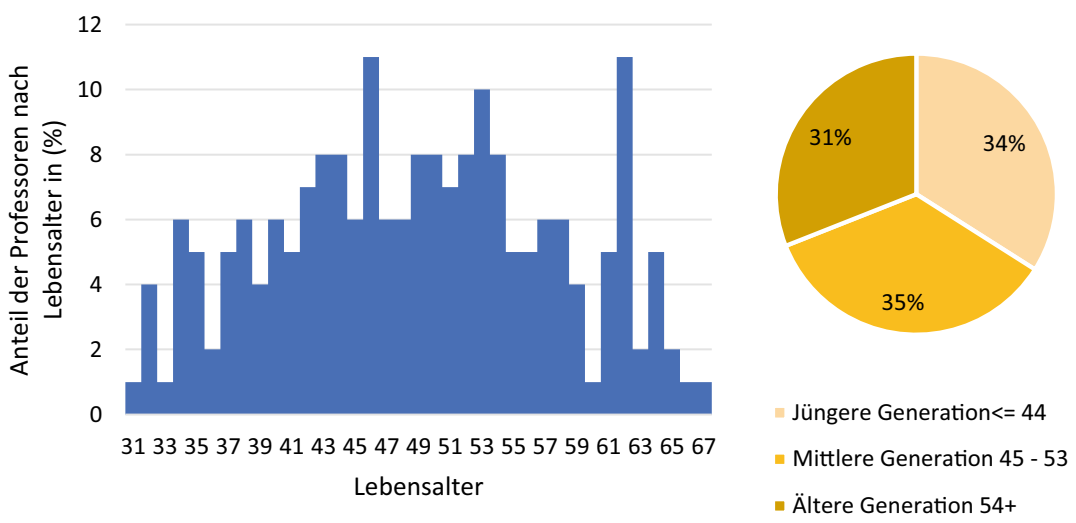

Abbildung 6.3 Alter der Professoren mit Migrationshintergrund und Altersgruppen. (Quelle: MOBIL 2012/2013 ( $\mathrm{N}=200)$ und $(\mathrm{N}=200)$ )

Hälfte bereits seit mehr als 20 Jahren oder gar in zweiter Generation in Deutschland. Hohe Zuwanderungszahlen zeigen sich speziell Mitte der 1990er-Jahre und nach 2002 (vgl. Abbildung 6.4).
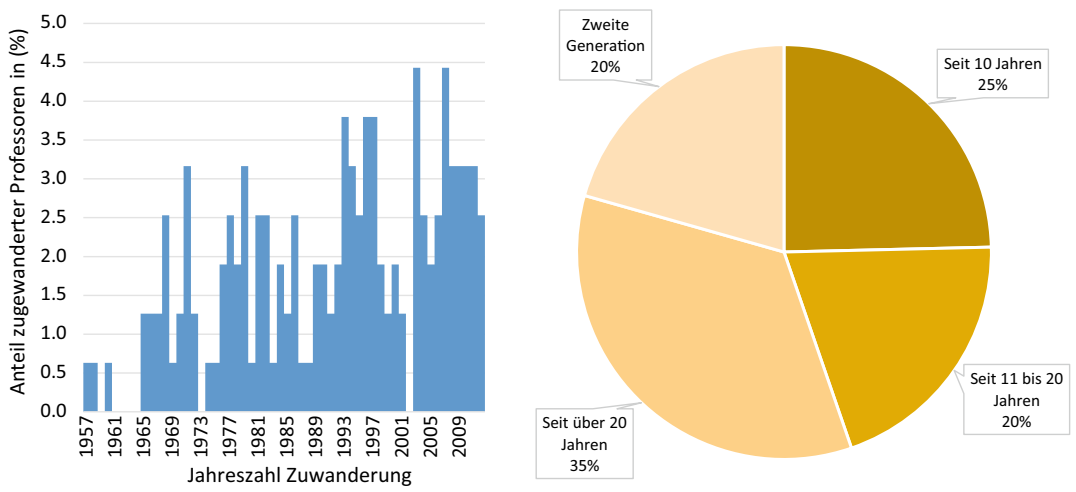

Abbildung 6.4 Jahreszahlen der Zuwanderung und Typologie zur Dauer in Deutschland. (Quelle: MOBIL 2012/2013 ( $\mathrm{N}=203$ ) und $(\mathrm{N}=158)$ )

Hinsichtlich der Altersstruktur zeigt sich, dass Professoren, die max. 10 Jahre in Deutschland leben, mit 43,8 mit Abstand am jüngsten sind. Professoren, die 
zwischen 11 und 20 Jahren in Deutschland leben liegen mit 48,7 Jahren fast exakt im Durchschnitt. Mit Abstand die älteste Gruppe bilden Professoren, die bereits seit über 20 Jahren in Deutschland leben mit 53,4 Jahren, während die zweite Generation mit 47,0 Jahren sogar etwas jünger ist als der Durchschnitt aller Professoren.

Zwischen der Klassifizierung nach Migrationstypen einerseits und nach Dauer in Deutschland andererseits bestehen große Überschneidungen. Dennoch führen beide Klassifizierungen nicht zu identischen Ergebnissen. Beispielsweise lebt fast jeder zehnte Professional Migrant bereits seit über 20 Jahren in Deutschland, während in etwa jeder neunte Student Migrant erst im Laufe der letzten 10 Jahren nach Deutschland gekommen ist (vgl. Tabelle 6.1).

\section{Tabelle 6.1}

Migrationstypen nach Aufenthaltsdauer in Deutschland

\begin{tabular}{l|l|l|l}
\hline $\begin{array}{l}\text { Jahre in } \\
\text { Deutchland }\end{array}$ & $\begin{array}{l}\text { Early } \\
\text { Migrants }\end{array}$ & $\begin{array}{l}\text { Student } \\
\text { Migrants }\end{array}$ & $\begin{array}{l}\text { Professional } \\
\text { Migrants }\end{array}$ \\
\hline Max. 10 Jahre & $0 \%$ & $11 \%$ & $62 \%$ \\
\hline 11-20 Jahre & $0 \%$ & $30 \%$ & $29 \%$ \\
\hline Über 20 Jahre & $37 \%$ & $59 \%$ & $9 \%$ \\
\hline $\begin{array}{l}\text { Zweite } \\
\text { Generation }\end{array}$ & $63 \%$ & $0 \%$ & $0 \%$ \\
\hline
\end{tabular}

Quelle: MOBIL 2012/2013 N = 199 p <0,000; p-value basiert auf $\chi^{2}$-Test

Im Wesentlichen sprechen zwei Argumente dafür, auf beide Klassifizierungen zurückzugreifen. Erstens gibt es bestimmte Fragestellungen, bei denen nur eine Klassifizierung aus theoretischer Perspektive plausibel erscheint. Zweitens hat sich empirisch gezeigt, dass sich bei einzelnen Fragestellungen signifikante Unterschiede exklusiv bei einer der beiden Klassifizierungen ergeben.

\subsubsection{Herkunftsländer nach der erweiterten Staatsangehörigkeit}

Nach dieser detaillierten Beschreibung der Migrationsverläufe sind im Folgenden die Herkunftsländer näher in den Blick zu nehmen. Um die Herkunftsländer der ausländischen Professoren und der deutschen Professoren mit Migrationshintergrund zu bestimmen, wird das erweiterte Staatsangehörigkeitskonzept verwendet. Das Konzept der erweiterten Staatsangehörigkeit wird durch das 
Statistische Bundesamt seit der Einführung des Migrationshintergrundes im Rahmen des Mikrozensus verwendet. Bei ausländischen Staatsangehörigen gilt die (erste) Staatsangehörigkeit, für Eingebürgerte die Staatsangehörigkeit vor der Einbürgerung oder ggf. die zweite Staatsangehörigkeit. Bei Personen mit Migrationshintergrund, die bereits als Deutsche geboren wurden, wird die derzeitige bzw. frühere Staatsangehörigkeit der Eltern ${ }^{6}$ herangezogen (Statistisches Bundesamt 2017a).

Insgesamt stammen die Professoren aus 40 unterschiedlichen Staaten. Die größten Herkunftsländer sind dabei Österreich, die Schweiz, die USA, die Niederlande und das Vereinigte Königreich, aus denen über die Hälfte (55\%) der Professoren kommen. Allein aus den beiden deutschsprachigen Nachbarländern Österreich und der Schweiz stammt fast ein Drittel (31 \%) der Professoren (vgl. Abbildung 6.5).

Nach einer kontinentalen Gruppierung kommen fast drei Viertel der Professoren aus europäischen Ländern (74\%) und weitere $15 \%$ aus Nordamerika und Australien. Die Anteile aus weiteren Weltregionen fallen indessen recht klein aus. Die größte Gruppe stellen hier noch Professoren aus Lateinamerika (6 \%), während Wissenschaftler aus Asien (3\%) und Afrika (1\%) kaum vertreten sind.

Interessant sind auch die großen Unterschiede hinsichtlich der Herkunftsregionen zwischen ausländischen Professoren und deutschen Professoren mit Migrationshintergrund. Die ausländischen Professoren kommen zu über $90 \%$ aus Österreich, der Schweiz, anderen westeuropäischen Ländern oder dem angelsächsischen Ausland. Demgegenüber zeigt sich bei den deutschen Professoren mit Migrationshintergrund eine größere regionale Diversität. Über $40 \%$ dieser Personen kommen aus Osteuropa, Lateinamerika, Asien und Afrika (vgl. Abbildung 6.6).

Eine weitere Differenzierung betrifft den Entwicklungsstand der Herkunftsländer. Die Hochschulforschung unterscheidet zwischen horizontaler Migration zwischen Ländern mit ähnlichem Entwicklungsstand und vertikaler Mobilität zwischen Ländern mit unterschiedlichem Entwicklungsstand (Teichler 2007). Als Grundlage wurde die Einteilung des Development Assistance Commitee (DAC) der Organisation für wirtschaftliche Zusammenarbeit und Entwicklung (OECD) genutzt, die zwischen Entwicklungs-/Schwellenländern und Industrieländern unterscheidet. Die in der DAC-Liste ${ }^{7}$ geführten Entwicklungs-/Schwellenländer

\footnotetext{
${ }^{6}$ Bei unterschiedlichen Staatsangehörigkeiten der Eltern wird die Staatsangehörigkeit der Mutter herangezogen.

${ }^{7}$ Die vollständige Liste ist abrufbar unter: https://www.bmz.de/de/zentrales_downloadarchiv/ Ministerium/ODA/DAC_Laenderliste_Berichtsjahre_2014_2016.pdf
} 


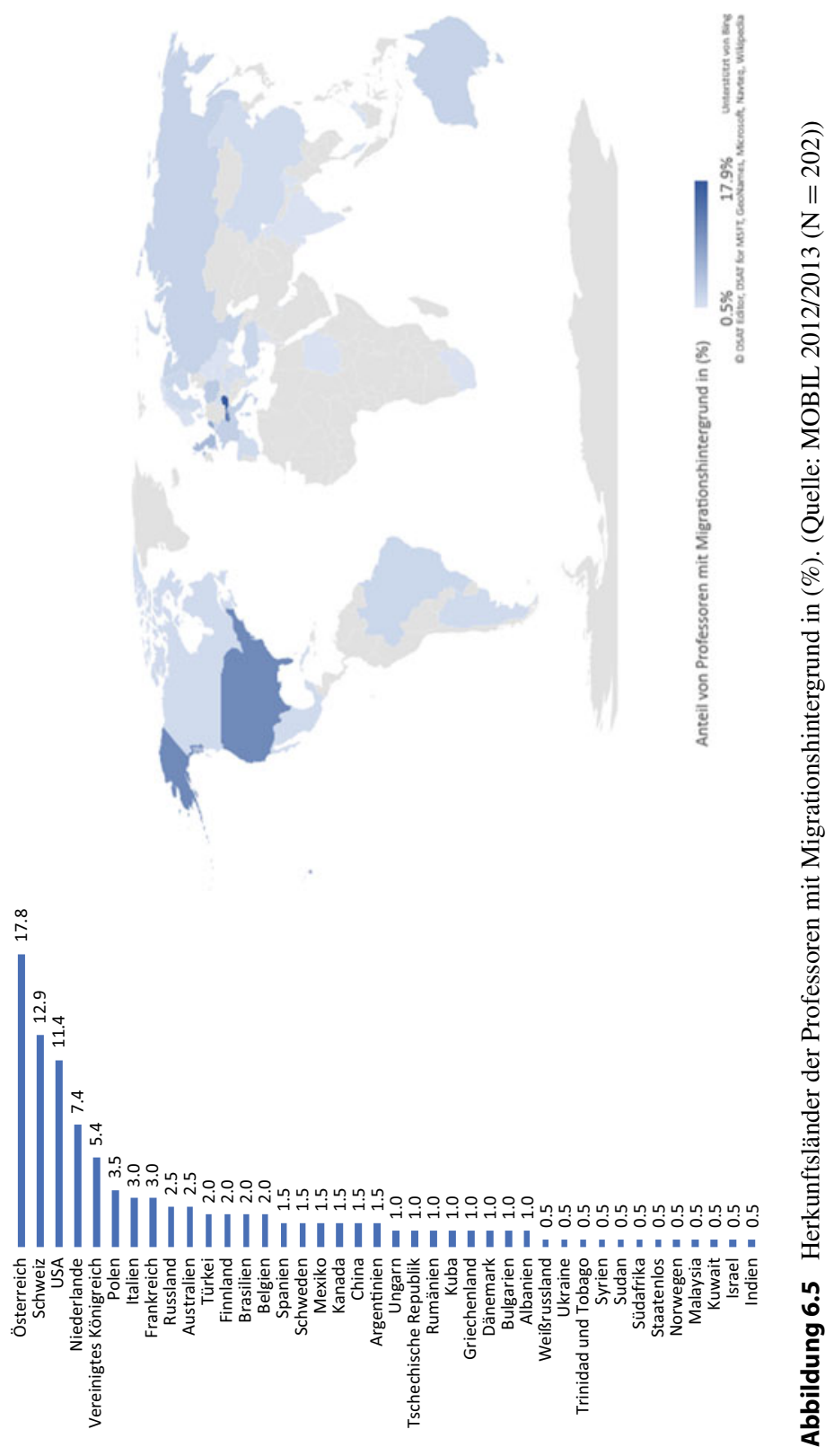




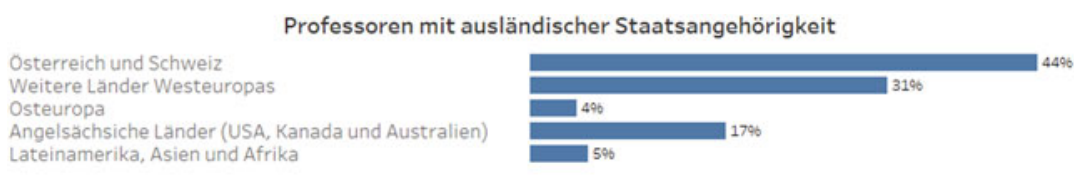

Deutsche mit Migrationshintergrund
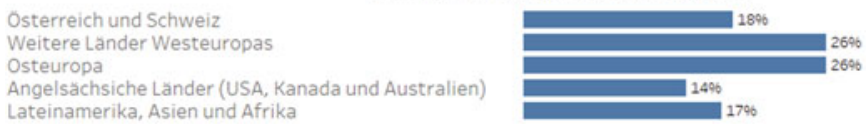

Gesamt

Osterreich und Schweiz

Weitere Lander Westeuropas

Osteuropa

Angelsachsiche Lander (USA, Kanada und Australien)

Lateinamerika, Asien und Afrika

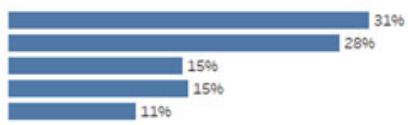

Abbildung 6.6 Unterschiede bei den Herkunftsregionen zwischen ausländischen Professoren und deutschen Professoren mit Migrationshintergrund. (Quelle: MOBIL 2012/2013; Ausländische Professoren: $\mathrm{N}=101$; Deutsche mit Migrationshintergrund $\mathrm{N}=101$, Gesamt $\mathrm{N}=202 \mathrm{p}<0,000$; $\mathrm{p}$-value basiert auf $\chi^{2}$-Test)

gehören allesamt zum Förderrahmen der Entwicklungszusammenarbeit. Die Einteilung des DAC wird auch vom Deutschen Akademischen Auslandsdienst und von der Publikationsreihe „Wissenschaft weltoffen“ verwendet.

Auf dieser Grundlage kommen 86 \% der Professoren aus Industrieländern und lediglich $14 \%$ aus Entwicklungs- und Schwellenländern. Diese verteilen sich im Einzelnen auf 14 Schwellen- und Entwicklungsländer, wobei allein die Hälfte der Gruppe aus der Türkei, Brasilien, Mexiko und China stammt. Der Großteil (79 \%) besitzt die deutsche Staatsangehörigkeit und viele von ihnen (43\%) sind als Student Migrants nach Deutschland gekommen.

\subsubsection{Arbeitsmigranten, (Spät-)Aussiedlern, Flüchtlinge und europäische Binnenmigration}

Im Folgenden sollen die gewonnenen Erkenntnisse über Zuwanderung und Herkunftsländer mit der Migrationsgeschichte Deutschlands seit den 1950er-Jahren (vgl. Abschnitt 2.1) in Bezug gesetzt werden. Konkret wird dabei der Frage nachgegangen, inwieweit die drei großen Zuwanderungsgruppen der Nachkriegszeit in Deutschland (Arbeitsmigranten, (Spät-) Aussiedler und Flüchtlinge) unmittelbar oder als Nachkommen unter den Professoren mit Migrationshintergrund zu 
finden sind. Zudem soll aufgrund der großen Zahl von Zuwanderern mit akademischem Abschluss aus dem europäischen Ausland seit Anfang der 2000er Jahre (vgl. Abschnitt 2.1.5) auch diese Gruppe näher betrachtet werden.

Erstens sollen die ca. 2,6 Millionen Arbeitsmigranten und ihre Nachkommen im Mittelpunkt stehen. Arbeitsmigranten sind im Kontext der Anwerbeabkommen zwischen 1955 und 1973 in die Bundesrepublik Deutschland zugewandert. Zudem gibt es in diesem Kontext eine große Zahl von Menschen, die speziell in den 1980er-Jahren im Zuge des Familiennachzugs nach Deutschland migrierten. Auch Zuwanderungsprozesse in die DDR auf der Grundlage von Regierungsabkommen Ende der 1970er-Jahre sollen dabei berücksichtigt werden. Über die Hälfte der knapp 200.000 ausländischen Beschäftigten wanderten aus Vietnam, Mosambik und Kuba in die DDR zu.

Zunächst lässt sich festhalten, dass unter den 203 befragten Professoren keine Person aus Vietnam, Mosambik und Kuba oder mit entsprechenden Wurzeln zu finden ist. Hinsichtlich der Anwerbeverträge der Bundesrepublik Deutschland lässt sich ebenfalls festhalten, dass aus vier der acht Staaten, mit denen bilaterale Abkommen geschlossen wurden, keine Professoren stammen. Weder aus Portugal noch aus Marokko oder Tunesien stammen Professoren im Sample. Auch aus Jugoslawien bzw. den nach dem Zerfall im Jahr 1991 entstandenen Nachfolgestaaten Serbien, Bosnien-Herzegowina, Slowenien, Kroatien, Mazedonien und Montenegro stammt keiner der Professoren. Die Anteile aus den vier weiteren Anwerbeländern fallen ebenfalls eher gering aus: Italien (3,0 \%), Türkei (2,0 \%), Spanien $(1,5 \%)$ und Griechenland $(1,0 \%)$. Von den insgesamt 15 Professoren aus Anwerbestaaten sind $40 \%$ bzw. sechs Personen erst nach 1990 zugewandert und haben somit keinen direkten Bezug mehr zur Zuwanderung im Kontext der Anwerbeabkommen. Eine Analyse der Bildungsabschlüsse und der Berufe der Eltern deuten darauf hin, dass es unter Vorbehalt maximal zwei Personen als Nachkommen der zunächst als Gastarbeiter titulierten Zuwanderer gelungen ist, eine erfolgreiche Laufbahn in Deutschland bis hin zur Professur zu durchlaufen.

Zweitens ist die Gruppe der Aussiedler/Spätaussiedler ${ }^{8}$ in den Blick zu nehmen. Seit 1950 sind über vier Millionen Aussiedler aus der ehemaligen UdSSR, Polen, Rumänien, der Tschechoslowakei und Nachfolgestaaten, aus Jugoslawien und Nachfolgestaaten, Ungarn sowie weiteren Gebieten nach Deutschland migriert. Mit Abstand die größte Zahl der Zuwanderer ist zwischen Ende der 1980er-Jahre und Mitte der 1990er-Jahre nach Deutschland gekommen.

Insgesamt stammen $15 \%$ der befragten Professoren aus Osteuropa. Aus Ländern, aus denen „Vertriebene“ als Aussiedler in Deutschland anerkannt wurden,

\footnotetext{
${ }^{8}$ Spätaussiedler umfasst alle Personen dieser Gruppe die erst ab 1993 zugewandert sind.
} 
stammen über $80 \%$ dieser Gruppe. Die insgesamt 24 Professoren stammen aus den folgenden Ländern: Polen (7), UdSSR-Nachfolgestaaten (7) [davon Russland (5), Weißrussland (1), Ukraine (1)], Tschechien (2), Ungarn (2), Rumänien (2), Bulgarien (2) und Albanien (2). Etwa ein Drittel von ihnen ist zwischen Ende der 1980er- und Mitte der 1990er-Jahre nach Deutschland zugewandert und über $80 \%$ haben die deutsche Staatsangehörigkeit. Die dargestellten Zahlen können indessen nur als „,notwendige Bedingung“ gesehen werden. Wie hoch der Anteil der Befragten, die tatsächlich als Aussiedler nach Deutschland gekommen sind, ist, lässt sich letztendlich auf dieser Datengrundlage nicht abschließend klären. Diese Erkenntnis kann zugleich als Hinweis verstanden werden, diesen Aspekt in zukünftigen Befragungen gesondert zu erheben.

Die dritte große Gruppe stellen Flüchtlinge dar. Seit dem sogenannten Sommer der Migration im Jahr 2015 ist das Flüchtlingsthema in Deutschland allgegenwärtig. Da die Befragung der Professoren aber bereits im Winter 2012/2013 stattgefunden hat, werden die jüngsten Migrationsbewegungen nicht in die Analyse mit einbezogen. Die erste große Debatte zur Flüchtlingszuwanderung fand zwischen Mitte der 1980er- und Mitte der 1990er-Jahre in Deutschland statt. Auslöser waren Flüchtlinge, die im Kontext des Zerfalls der UdSSR und des Jugoslawienkrieges nach Deutschland kamen. Zwischen 2003 und 2012 wurden Asylanträge vor allem von Menschen aus folgenden Ländern gestellt: Afghanistan, Aserbaidschan, Bosnien und Herzegowina, China, Indien, Irak, Iran, Kosovo, Libanon, Mazedonien, Nigeria, Pakistan, Russische Föderation, Serbien, Montenegro, Somalia, Syrien, Türkei und Vietnam (Bundesamt für Migration und Flüchtlinge 2013, S. 19).

Weder aus Jugoslawien bzw. den Nachfolgestaaten noch aus den meisten der genannten Länder finden sich Professoren in dem Sample. Lediglich eine kleine Zahl von Professoren aus China, Indien und der Türkei ist unter den befragten Professoren. Auch hier lässt sich aber nicht abschließend klären, inwieweit Flüchtlinge oder Nachkommen von Flüchtlingen überhaupt im Sample vertreten sind. Die Analysen verweisen aber wiederum darauf, dass, wenn überhaupt, nur eine sehr kleine Zahl unter den Professoren mit Migrationshintergrund zu finden ist.

Zusammenfassend lässt sich konstatieren, dass die Analysen darauf hindeuten, dass die drei großen Zuwanderungsgruppen in der Migrationsgeschichte Deutschlands seit dem Zweiten Weltkrieg kaum unter Professoren mit Migrationshintergrund vertreten sind. Insbesondere die Nachkommen von Arbeitsmigranten sowie Flüchtlinge und ihre Nachkommen sind, wenn überhaupt, nur marginal unter Professoren mit Migrationshintergrund vertreten. Insbesondere im Kontext der großen Flüchtlingszuwanderung der letzten Jahre sollte in kommenden 
Befragungen systematisch erhoben werden, wie stark Migranten der drei großen Zuwanderungsgruppen repräsentiert sind.

Deutlich stärker spiegelt sich in den Ergebnissen hingegen die verstärkte Zuwanderung von Hochqualifizierten aus dem europäischen Ausland zwischen 2000 und dem Befragungszeitpunkt 2012 nach Deutschland wider (vgl. Abschnitt 2.1.5). Allein ein Viertel der Professoren mit Migrationshintergrund ist in den letzten 10 Jahren zugewandert, davon $78 \%$ aus dem europäischen Ausland. Es ließe sich hier vermuten, da zwischen 2000 und 2012 eine große Zahl von Zuwanderern mit akademischen Abschlüssen aus Ost- und Südeuropa nach Deutschland gekommen ist (vgl. Abschnitt 2.1.5), dass sich dieser Trend auch in den Herkunftsländern der in dem Zeitraum zugewanderten Professoren widerspiegelt. Allerdings findet auch in diesem Zeitraum in erster Linie eine Zuwanderung aus Österreich (33\%) und der Schweiz (23\%) statt, es folgen Professoren aus den Niederlanden mit $10 \%$. Zudem für die Gruppe der aus dem europäischen Ausland zugewanderten Professoren, dass sie seltener die deutsche Staatsangehörigkeit besitzen. Möglicherweise liegt dies darin begründet, dass die Relevanz der deutschen Staatsangehörigkeit für die Arbeits- und Aufenthaltsgenehmigung aufgrund der häufig vorliegenden EU-Staatsangehörigkeit deutlich kleiner ausfällt. Professoren aus dem europäischen Ausland stellen fast Dreiviertel der zugewanderten Professoren. Dementsprechend sollte in Erwägung gezogen werden, in zukünftigen Studien Kontextbedingungen, Prozesse und Hintergründe innerhalb Europas wie bspw. den Einfluss der Europäischen Union, des europäischen Hochschulraums oder auch europäischer Austauschprogramme (wie Erasmus) im Kontext der Migration von Professoren näher zu erforschen.

\subsubsection{Muttersprache}

Neben Zuwanderungsalter und Herkunftsland war auch die Muttersprache der Professoren Teil der Erhebung. Insbesondere im Feld der interkulturellen Bildungsforschung wird der Zusammenhang von Sprachentwicklung und Bildung seit mehreren Jahren diskutiert. Studien verweisen dabei auf ein großes Potential eines stärker bilingualen Unterrichts (Gogolin 2010) (Abbildung 6.7).

Interessant ist in diesem Zusammenhang, dass von über einem Viertel der Professoren mit Migrationshintergrund zwei oder drei Muttersprachen genannt werden. Überraschend erscheint zunächst, dass über die Hälfte der Professoren mit Migrationshintergrund Deutsch als eine Muttersprache angibt. Der auf den ersten Blick sehr hohe Anteil an Deutsch-Muttersprachlern lässt sich in erster 


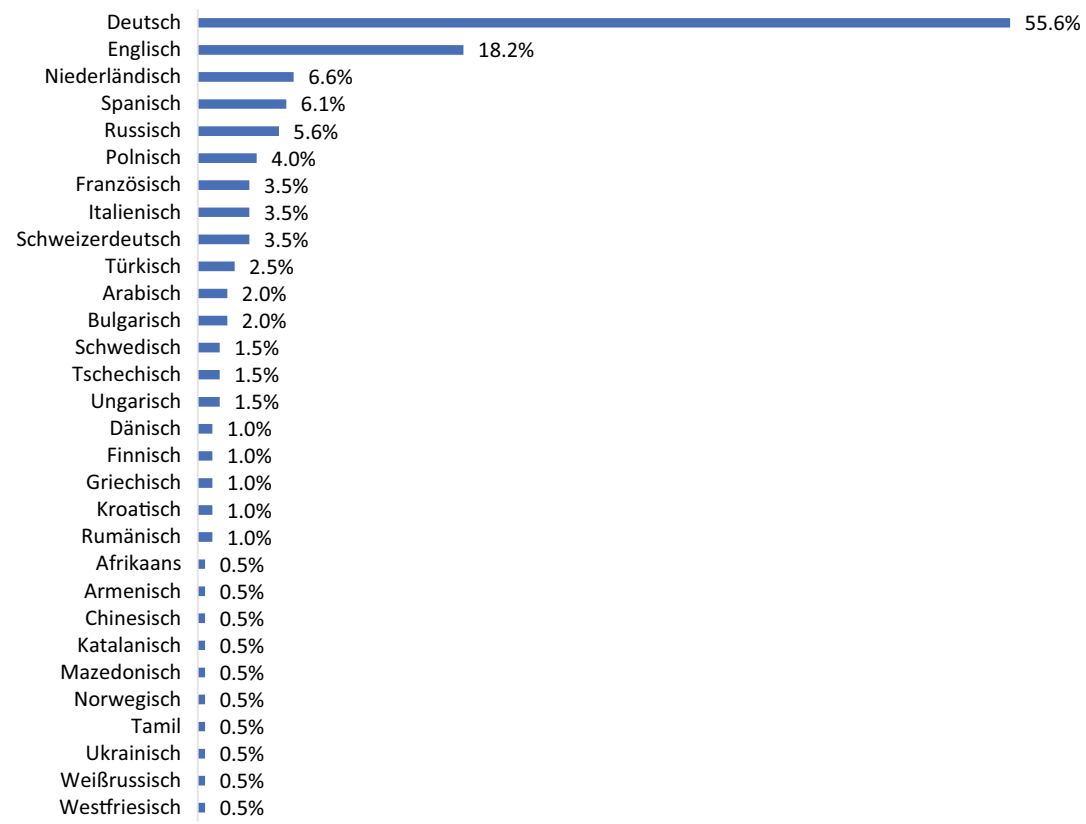

Abbildung 6.7 Muttersprachen der Professoren mit Migrationshintergrund. (Quelle: MOBIL 2012/2013 ( $\mathrm{N}=198$ ) [Mehrfachantworten])

Linie auf das Zuwanderungsalter und die Sprache des Herkunftslandes zurückführen. Erstens verdient dabei die Gruppe der Early Migrants, die bereits in Deutschland geboren sind oder als Kinder und Jugendliche nach Deutschland gekommen sind, Erwähnung. Der Anteil der Deutsch-Muttersprachler liegt in dieser Gruppe bei $91 \%$. Zweitens ist die große Zuwanderung aus den beiden deutschsprachigen Nachbarländern zu berücksichtigen. Deutsch-Muttersprachler unter den Student Migrants und Professional Migrants stammen zu $80 \%$ aus Österreich oder der Schweiz. Weitere häufig genannte Muttersprachen sind Englisch, Niederländisch, Spanisch, Russisch, Polnisch, Italienisch, Französisch und Türkisch (vgl. Abbildung 6.6). Die Darstellungen bieten einen interessanten Einblick in die unterschiedlichen sprachlichen Hintergründe der Professoren. Dennoch bleibt der Begriff der Muttersprache definitorisch schwer zu fassen und für zukünftige Befragungen sollte stärker der Akzent darauf gelegt werden, welche 
Sprachen in der Bildungs- und Berufsbiographie eine Rolle gespielt haben und welche Sprachkompetenzen erworben wurden.

\subsubsection{Geschlecht}

Neben den Migrationsmerkmalen spielen demographische Merkmale in diesem Zusammenhang eine wichtige Rolle. Insbesondere Fragen der Chancengerechtigkeit werden in der Hochschulforschung bisher vor allem hinsichtlich des Geschlechts und der sozialen Herkunft untersucht.

Ein zentraler Indikator für die Teilhabechancen von Frauen in der Wissenschaft ist die Entwicklung des Frauenanteils in der Professorenschaft. Hier zeigt sich, dass Frauen zu Beginn der 1990er-Jahre mit einem Anteil von 6,5\% in Spitzenpositionen der Wissenschaft kaum existent waren. Seitdem ist es gelungen, den Anteil auf 23,4 \% mehr als zu verdreifachen. Allerdings findet sich auch heute in noch nicht einmal jeder vierten Professur eine Frau. Dabei ist notwendigerweise nach Fachbereichen und Fächergruppen zu differenzieren, da sich hier erhebliche Unterschiede ergeben. Beispielsweise liegen die Frauenanteile unter Professoren in der Sonderpädagogik, in den Sozialwissenschaften oder in den Gesundheitswissenschaften bei fast $50 \%$ (vgl. auch Hüther und Krücken 2016). Demgegenüber verbleiben die Frauenanteile in den Ingenieurund Naturwissenschaften bei deutlich unter $20 \%$ (vgl. Statistisches Bundesamt 2018b).

Der Frauenanteil im Rahmen der MOBIL-Studie liegt bei $34 \%$ und damit deutlich über Frauenanteil innerhalb der gesamten Professorenschaft in Deutschland sowie der Professorenschaft in Berlin und Hessen (vgl. Statistisches Bundesamt 2018b). Ein besonders hoher Frauenanteil zeigt sich in der jüngeren Generation unter 45 Jahren (44\%). Keine signifikanten Geschlechterunterschiede zeigen sich hingegen zwischen den verschiedenen Migrationstypen. Die Frage, inwieweit Unterschiede zwischen Professorinnen und männlichen Professoren mit Migrationshintergrund sichtbar werden, wird im Rahmen der Arbeit wiederholt aufgegriffen.

\subsubsection{Soziale Herkunft}

Bei der Diskussion über Chancengleichheit im Hochschulwesen ist neben dem Geschlecht auch die soziale Herkunft von zentraler Bedeutung. Die Frage, ob und in welchem Maße es Kindern aus bildungsfernen Schichten gelingt, einen 
Hochschulabschluss zu erwerben und darüber hinaus möglicherweise auch eine wissenschaftliche Karriere bis hin zur Professur zu verfolgen, kann exemplarisch über die Chancen sozialer Mobilität innerhalb einer Gesellschaft Aufschluss geben. Um die soziale Herkunft zu beschreiben, wird zunächst die ökonomische Situation in der Kindheit dargestellt und dann im zweiten Schritt die Bildungsabschlüsse der Eltern.

Grundsätzlich gibt es eine Vielzahl unterschiedlicher Ansätze, um soziale Herkunft $\mathrm{zu}$ messen. Als ein zentraler Indikator hat sich der Bildungsabschluss etabliert. Dazu werden insbesondere mögliche akademische Abschlüsse der Eltern in den Blick genommen. Zudem wurden die Teilnehmer hinsichtlich der ökonomischen Verhältnisse ihres Elternhauses in der Kindheit befragt.

Hinsichtlich der ökonomischen Verhältnisse treffen $28 \%$ der Befragten die Aussage, dass das Geld in ihrer Familie eher knapp war, während fast zwei Drittel der Befragten ihre Familie der damaligen Mittelschicht zuordnen. Lediglich eine kleine Gruppe von $8 \%$ gibt an, in wohlhabenden Verhältnissen aufgewachsen zu sein. Aus einer Vielzahl empirischer Studien geht indessen hervor, dass Personen, die vom Einkommen bzw. Vermögen her eigentlich eindeutig zu einer oberen gesellschaftlichen Gruppe gehören, sich in Befragungen nur selten auch selbst dort verorten, sondern sich eher der Mittelschicht zuordnen. Vor diesem Hintergrund ist möglicherweise auch der geringe Anteil von Professoren aus wohlhabenden Verhältnissen zu erklären.

Hinsichtlich der Bildungsherkunft zeigt sich, dass $55 \%$ der Väter und $40 \%$ der Mütter einen Hochschulabschluss besitzen. Einen Promotionsabschluss haben $22 \%$ der Väter und $6 \%$ der Mütter erreicht. Fast zwei Drittel der Professoren haben einen Elternteil mit akademischem Abschluss, während bei über einem Viertel der Teilnehmer beide Eltern einen Hochschulabschluss vorweisen können. Dementsprechend liegt der Anteil an Professoren, die innerhalb ihrer Familie als Erste einen akademischen Werdegang eingeschlagen haben und damit zur sogenannten First Generation gehören, bei lediglich 37 \%. Aus anderen Studien ist bekannt, dass es sich bei Professoren generell um eine sozial hochselektive Gruppe handelt, wobei auch in der Studie von Möller (2017b) zugewanderte Professoren noch deutlich seltener (46\%) aus nichtakademischen Elternhäusern kommen als Professoren, die in Deutschland geboren wurden (62\%).

Auffällig ist der geringe Anteil der First Generation unter Professoren aus Entwicklungs- und Schwellenländern ${ }^{9}$. Nur ca. ein Viertel der Professoren aus diesen Ländern (26\%) kommt aus einem nichtakademischen Elternhaus. Auch unter Berücksichtigung des generell sehr geringen Professorenanteils aus

\footnotetext{
${ }^{9}$ Aufgrund der geringen Fallzahl $(\mathrm{n}=23)$ ist dieser Befund statistisch nicht signifikant.
} 
Entwicklungs- und Schwellenländern muss festgehalten werden, dass für Menschen aus „einfachen“ Verhältnissen in Entwicklungs- und Schwellenländern Karriereverläufe, die zu einer Professur an einer deutschen Hochschule führen, den absoluten Ausnahmefall darstellen. Unter Professorinnen liegt der Anteil der First Generation höher $(43 \%)$ als unter männlichen Professoren $(33 \%)^{10}$. Dieser Befund überrascht zunächst, da die Vermutung naheliegt, dass insbesondere Frauen aus nichtakademischen Elternhäusern, in denen die beiden Ungleichheitsdimensionen Geschlecht und soziale Herkunft zusammenkommen, besondere Schwierigkeiten haben dürften, eine Professur zu erlangen. Der sozial selektive Zugang für Professoren mit Migrationshintergrund wird zudem auch darin sichtbar, dass in mehr als einem Fünftel der Fälle (22\%) bereits die Eltern an der Hochschule tätig waren. Davon waren $70 \%$ bereits als Professoren beschäftigt.

\subsubsection{Hochschulart}

Hinsichtlich der Hochschularten ist zwischen Universitäten und Fachhochschulen zu differenzieren (vgl. Abschnitt 2.2). In etwa jeder fünfte Professor mit Migrationshintergrund arbeitet an einer Fachhochschule (21\%), während der Anteil an Universitäten dementsprechend bei $79 \%$ liegt. Insgesamt $8 \%$ der Professoren mit Migrationshintergrund arbeiten an Kunst- und Musikhochschulen. Professoren an Kunst- und Musikhochschulen werden - wie im Übrigen auch in der amtlichen Statistik und in anderen Studien - aufgrund der geringen Fallzahl mit zur Gruppe der Professoren an Universitäten gezählt.

Deutliche Unterschiede zeigen sich zwischen den Migrationstypen. Während $30 \%$ der Early Migrants und $28 \%$ der Student Migrants an Fachhochschulen arbeiten, liegt der Anteil an Fachhochschulprofessoren unter Professional Migrant bei lediglich $6 \%$. Aus anderen Studien ist bekannt, dass Professoren an Fachhochschulen häufiger aus nichtakademischen Elternhäusern stammen (Schomburg et al. 2012, S. 27). Die Ergebnisse zeigen, dass der Anteil an Professoren der First Generation an Fachschulen mit $38 \%$ sich kaum vom Anteil an Universitäten $(36 \%)$ unterscheidet. Es lässt sich festhalten, dass Fachhochschulen insbesondere für die wissenschaftliche Karriere von Early Migrants und der zweiten Zuwanderergeneration eine wichtige Option darstellt, zugleich aber nicht wie bei Studierenden in besonderer Weise sozial durchlässig ist. Im Zusammenhang mit dem hohen Anteil an Early Migrants an Fachhochschulen erklärt sich auch die Tatsache, dass deutsche Professoren mit Migrationshintergrund (33\%)

${ }^{10}$ Dieser Unterschied ist statistisch nicht signifikant. 
deutlich häufiger an Fachhochschulen zu finden sind als Professoren mit ausländischer Staatsangehörigkeit (9\%). Auffällig ist zudem, dass Professoren aus Entwicklungs- und Schwellenländen an Fachhochschulen (14\%) einen deutlich kleineren Anteil ausmachen als an Universitäten $(23 \%)^{11}$.

\subsubsection{Besoldungsgruppe}

Bei den Besoldungsgruppen der Professoren lässt sich zwischen der alten CBesoldung und der neuen W-Besoldung, die Anfang der 2000er Jahre in Kraft getreten ist, differenzieren (vgl. Abschnitt 2.3). An dieser Stelle ist zwischen folgenden drei Gruppen zu unterscheiden:

- W1-Professur (entspricht der Juniorprofessur) (14\%)

- W2-/C3-/C2-Professur (43\%)

- W3-/C4-Professur (43\%)

Die Besoldungsgruppen unterscheiden sich je nach Hochschulart grundlegend. An Fachhochschulen gibt es keine Juniorprofessuren und W3-/C4-Professuren gibt es nur in seltenen Ausnahmefällen. Unter den Befragten gab lediglich ein Fachhochschulprofessor an, eine W3-/C4-Besoldung zu erhalten. Ansonsten sind alle Professoren an Fachhochschulen einer der Besoldungsgruppen W2/C3/C2 zugeordnet. Auf dieser Grundlage wurden die folgenden vier Gruppen gebildet $^{12}$ :

- Juniorprofessuren (Universität) (14\%)

- Fachhochschulprofessuren (22\%)

- W2-/C3-/C2-Professuren (Universität) $(22 \%)^{13}$

- W3-/C4-Professuren (Universität) (43\%)

Das Durchschnittsalter liegt für die Juniorprofessoren bei 37 Jahren, für die W2-/C3-/C2-Professoren an Universitäten bei 49 Jahren und sowohl für die Fachhochschulprofessoren als auch für die W3-/C4-Professoren an Universitäten bei 51 Jahren. Die befragten Professoren im Rahmen der CAP-Erhebung waren an

\footnotetext{
${ }^{11}$ Dieses Ergebnis ist statistisch nicht signifikant.

${ }^{12}$ Die Abweichung von $100 \%$ ergibt sich aufgrund der vorgenommenen Rundungen.

${ }^{13} \mathrm{C} 2$-Besoldung finden sich ausschließlich an Kunst- und Fachhochschulen. Da Professoren der Kunsthochschulen aufgrund der kleinen Fallzahl auch mit zur Hochschulart Universität gerechnet werden, ist die Differenzierung W2-/C3-/C2-Professur an Universitäten dennoch sinnvoll.
} 
Universitäten 53 Jahre und an Fachhochschulen 52 Jahre; Juniorprofessoren waren durchschnittlich ebenfalls 37 Jahre (Teichler et al. 2017). Der Vergleich deutet darauf hin, dass Professoren mit Migrationshintergrund etwas jünger sind als ihre Kollegen ohne Migrationshintergrund.

Auffällige Unterschiede hinsichtlich der Besoldungsgruppen zeigen sich zwischen Professorinnen und männlichen Professoren. Jede vierte Professorin mit Migrationshintergrund arbeitet als Juniorprofessorin, während lediglich $8 \%$ der befragten männlichen Professoren eine Juniorprofessur innehaben. Demgegenüber bekleidet fast jeder zweite Professor eine Universitätsprofessur der höchsten Besoldungsstufe, während weniger als ein Drittel der Professorinnen auf einer solchen Professur zu finden ist. Nur geringfügige Unterschiede zeigen sich hingegen bei dem Anteil an Professoren der First Generation hinsichtlich der verschiedenen Besoldungsgruppen.

Ein positiver Aspekt dieser Ergebnisse kann darin zu sehen sein, dass ein Frauenanteil von $59 \%$ unter den Juniorprofessuren eine Perspektive aufzeigt, dass Geschlechterdisparitäten in Zukunft weiter abnehmen dürften. Andererseits weist der geringe Frauenanteil von lediglich $23 \%$ in der höchsten Besoldungsgruppe darauf hin, dass trotz des generell höheren Frauenanteils unter Professoren mit Migrationshintergrund weiterhin deutliche Ungleichheiten bestehen. Ähnliche Unterschiede zeigen sich zwischen Professoren aus Entwicklungs/Schwellenländern einerseits und Industrieländern andererseits. Während in etwa jeder vierte Juniorprofessor aus einem Entwicklungs- oder Schwellenland stammt, liegt der Anteil unter Universitätsprofessoren der höchsten Besoldungsstufe bei unter $8 \%$.

Zusammenfassend lässt sich festhalten, dass die strukturelle Zusammensetzung der Juniorprofessoren durchaus Hoffnung macht, dass in Zukunft eine größere Diversität in Bezug auf Geschlecht und Herkunftsregion unter Professoren mit Migrationshintergrund zu finden sein wird. Kritisch berücksichtigt werden sollte hingegen, dass der Anteil von Professoren der First Generation hier mit $30 \%$ noch etwas geringer ausfällt als in der gesamten Professorenschaft. Zugleich muss auch konstatiert werden, dass beim Zugang zur höchsten Besoldungsgruppe für Frauen einerseits und für Menschen aus wirtschaftlich benachteiligten Staaten andererseits besondere Hürden sichtbar werden (vgl. ausführlich Engel 2017). Bei den Migrationstypen zeigt sich, dass Professional Migrants mit Abstand die größte Gruppe unter W3-/C4-Professoren an Universitäten stellen. Dieser Anteil der Professional Migrants von fast $50 \%$ lässt sich möglicherweise auch damit erklären, dass in dieser Besoldungsgruppe am häufigsten eine internationale Rekrutierung von Professoren stattfindet. 


\subsubsection{Fächergruppe}

Die Kategorisierung unterschiedlicher Fachbereiche wurde zum einen nach der DFG-Systematik der Fachgebiete und Wissenschaftsbereiche und zum anderen in Anlehnung an die Lehr- und Forschungsbereiche und Fachgebiete des Statistischen Bundesamtes vorgenommen ${ }^{14}$. Dabei wird für den mit Abstand größten Wissenschaftsbereich der Geistes- und Sozialwissenschaften zwischen den Fachgebieten Geisteswissenschaften und Sozial-/Verhaltenswissenschaften differenziert. Kunst und Kunstwissenschaften, die einen Teil der Geisteswissenschaften darstellen, werden aufgrund der Gruppengröße und Spezifik separat betrachtet. Des Weiteren werden die nach der DFG-Systematik gängigen Wissenschaftsbereiche der Lebenswissenschaften, Naturwissenschaften und Ingenieurwissenschaften näher beleuchtet. Über die Hälfte der Professoren ist im Bereich der Geistes- und Sozialwissenschaften beschäftigt.

Die größte Gruppe Professoren ist in den Sozial- und Verhaltenswissenschaften zu finden, wovon fast die Hälfte aus Professoren der Wirtschaftswissenschaften besteht. In den Geisteswissenschaften stellt die Anglistik/Amerikanistik die größte Zahl an Professoren mit Migrationshintergrund. Im Bereich Kunst/Kunstwissenschaften arbeiten allein $16 \%$ aller befragten Professoren. Die größte Zahl an Professoren mit Migrationshintergrund arbeitet hier im Lehr- und Forschungsbereich Musik. Der Wissenschaftsbereich Lebenswissenschaften setzt sich primär aus Biologen und Humanmedizinern zusammen. In den Naturwissenschaften bilden die größten Lehr- und Forschungsbereiche die Physik/Astronomie und die Informatik. Die Elektrotechnik stellt hingegen den größten Lehr- und Forschungsbereich innerhalb der Ingenieurwissenschaften (vgl. Tabelle 6.2).

Nach Möglichkeit wird im Folgenden versucht, bei fächerspezifischen Fragestellungen eine Differenzierung anhand der sechs Gruppen vorzunehmen. Wenn aufgrund der Fallzahl eine derart differenzierte Form der Analyse nicht möglich sein sollte, werden die Geistes- und Sozialwissenschaften unter dem Label „Humanities “ und die Natur-, Lebens-, und Ingenieurwissenschaften unter dem Label „Science“ zusammengefasst.

\footnotetext{
${ }^{14}$ Die Fächersystematik beschreibt die Konkordanz zwischen der Systematik der Fächergruppen, Lehr- und Forschungsbereiche und Fachgebiete des Statistischen Bundesamtes und der DFG-Systematik der Fachgebiete und Wissenschaftsbereiche. Nähere Informationen: https://www.dfg.de/download/pdf/dfg_im_profil/gremien/fachkollegien/amt speriode_2016_2019/fachsystematik_2016-2019_de_grafik.pdf.
} 


\begin{tabular}{|c|c|c|c|c|c|c|c|}
\hline & $\frac{\circ}{m}$ & $\frac{\stackrel{\circ}{i}}{i}$ & $\begin{array}{l}\stackrel{0}{0} \\
\dot{0} \\
-\end{array}$ & 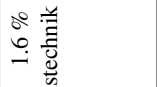 & $\begin{array}{l}\stackrel{\circ}{0} \\
\stackrel{-}{-}\end{array}$ & $\begin{array}{l}\stackrel{\circ}{0} \\
\stackrel{-}{-}\end{array}$ & $\begin{array}{l}20 \\
n ? \\
0\end{array}$ \\
\hline \multirow[t]{2}{*}{ 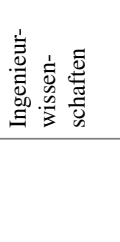 } & $\begin{array}{l}\frac{1}{0} \\
\frac{0}{0} \\
\frac{0}{0} \\
\frac{0}{\square} \\
I\end{array}$ & 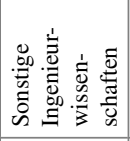 & 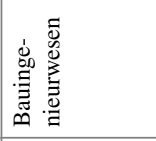 & 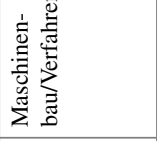 & 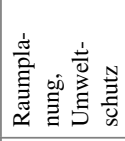 & 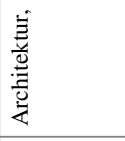 & 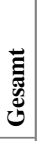 \\
\hline & $\begin{array}{l}\stackrel{2}{2} \\
\stackrel{9}{r}\end{array}$ & $\begin{array}{l}\stackrel{\circ}{0} \\
\text { m) }\end{array}$ & $\begin{array}{l}\stackrel{0}{1} \\
\stackrel{+}{f}\end{array}$ & $\begin{array}{l}\stackrel{0}{0} \\
0 \\
i\end{array}$ & $\begin{array}{l}\frac{\partial}{i} \\
\vec{i}\end{array}$ & $\begin{array}{l}\stackrel{0}{0} \\
\stackrel{0}{-}\end{array}$ & $\begin{array}{l}\stackrel{0}{0} \\
\stackrel{\dot{d}}{d}\end{array}$ \\
\hline 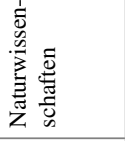 & 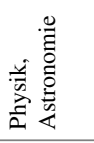 & 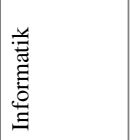 & $\begin{array}{l}\text { 弟 } \\
\text { 离 } \\
\text { 壳 }\end{array}$ & 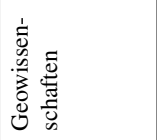 & 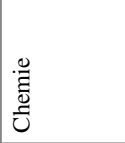 & 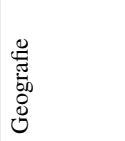 & 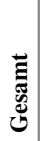 \\
\hline & $\stackrel{\stackrel{\circ}{\circ}}{\stackrel{f}{f}}$ & $\begin{array}{l}\stackrel{\circ}{r} \\
\stackrel{m}{r}\end{array}$ & $\begin{array}{l}\stackrel{0}{0} \\
\stackrel{-}{-}\end{array}$ & $\begin{array}{l}80 \\
n ? \\
0\end{array}$ & $\begin{array}{l}\stackrel{0}{2} \\
\stackrel{a}{ }\end{array}$ & $\curvearrowright$ & \\
\hline 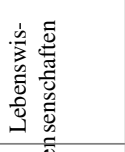 & $\begin{array}{l}\frac{0}{50} \\
\frac{0}{0} \\
\frac{0}{0}\end{array}$ & 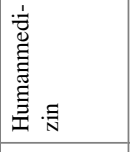 & 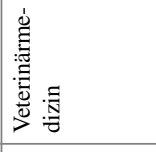 & 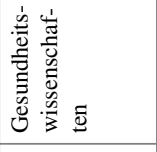 & 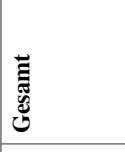 & $\mathbf{z}$ & \\
\hline 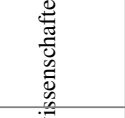 & $\begin{array}{l}\stackrel{\circ}{\circ} \\
\stackrel{+}{f}\end{array}$ & $\begin{array}{l}\stackrel{\circ}{r} \\
\dot{m}\end{array}$ & $\frac{\circ}{m}$ & $\frac{\circ}{i}$ & $\begin{array}{l}\stackrel{\circ}{0} \\
\stackrel{-}{\circ}\end{array}$ & $\begin{array}{l}\stackrel{0}{0} \\
\stackrel{-}{-}\end{array}$ & 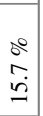 \\
\hline 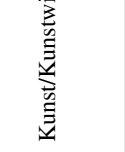 & $\frac{\frac{4}{5}}{\sum^{2}}$ & 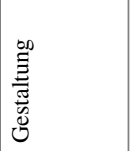 & 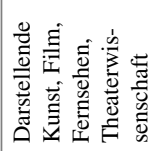 & 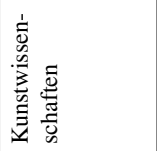 & 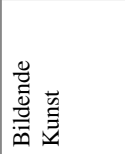 & 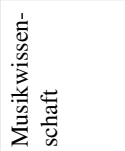 & 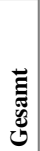 \\
\hline & $\begin{array}{l}\stackrel{0}{0} \\
\stackrel{-}{=}\end{array}$ & $\begin{array}{l}\stackrel{\circ}{ } \\
\stackrel{+}{f}\end{array}$ & $\begin{array}{l}\stackrel{0}{0} \\
\dot{b} \\
i\end{array}$ & $\frac{\delta^{\circ}}{i}$ & $\begin{array}{l}\stackrel{\circ}{0} \\
\stackrel{-}{-}\end{array}$ & $\begin{array}{l}\stackrel{0}{0} \\
\stackrel{0}{-}\end{array}$ & $\begin{array}{l}\text { so } \\
n \\
0\end{array}$ \\
\hline 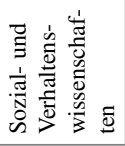 & 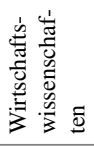 & 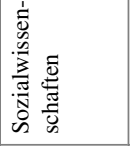 & $\begin{array}{l}\frac{0}{50} \\
\frac{0}{0} \\
\frac{0}{0} \\
0 \\
0\end{array}$ & 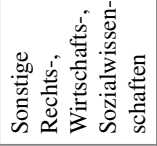 & 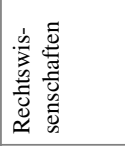 & 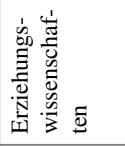 & 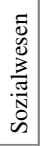 \\
\hline & $\begin{array}{l}\stackrel{\circ}{0} \\
\text { bo } \\
i\end{array}$ & $\begin{array}{l}\delta^{\circ} \\
\vec{i}\end{array}$ & $\frac{\partial^{\circ}}{i}$ & $\begin{array}{l}\stackrel{\circ}{0} \\
\stackrel{-}{0}\end{array}$ & $\begin{array}{l}\stackrel{0}{0} \\
\stackrel{0}{-}\end{array}$ & $\begin{array}{l}\stackrel{0}{0} \\
\stackrel{0}{0}\end{array}$ & $\begin{array}{l}\stackrel{0}{0} \\
0 \\
0 \\
-1\end{array}$ \\
\hline 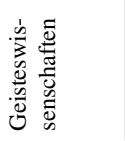 & 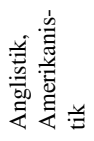 & 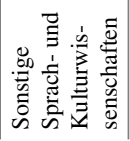 & 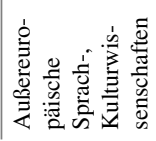 & 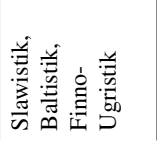 & 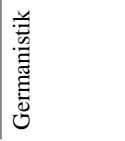 & 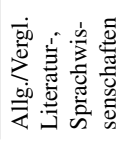 & 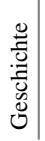 \\
\hline
\end{tabular}




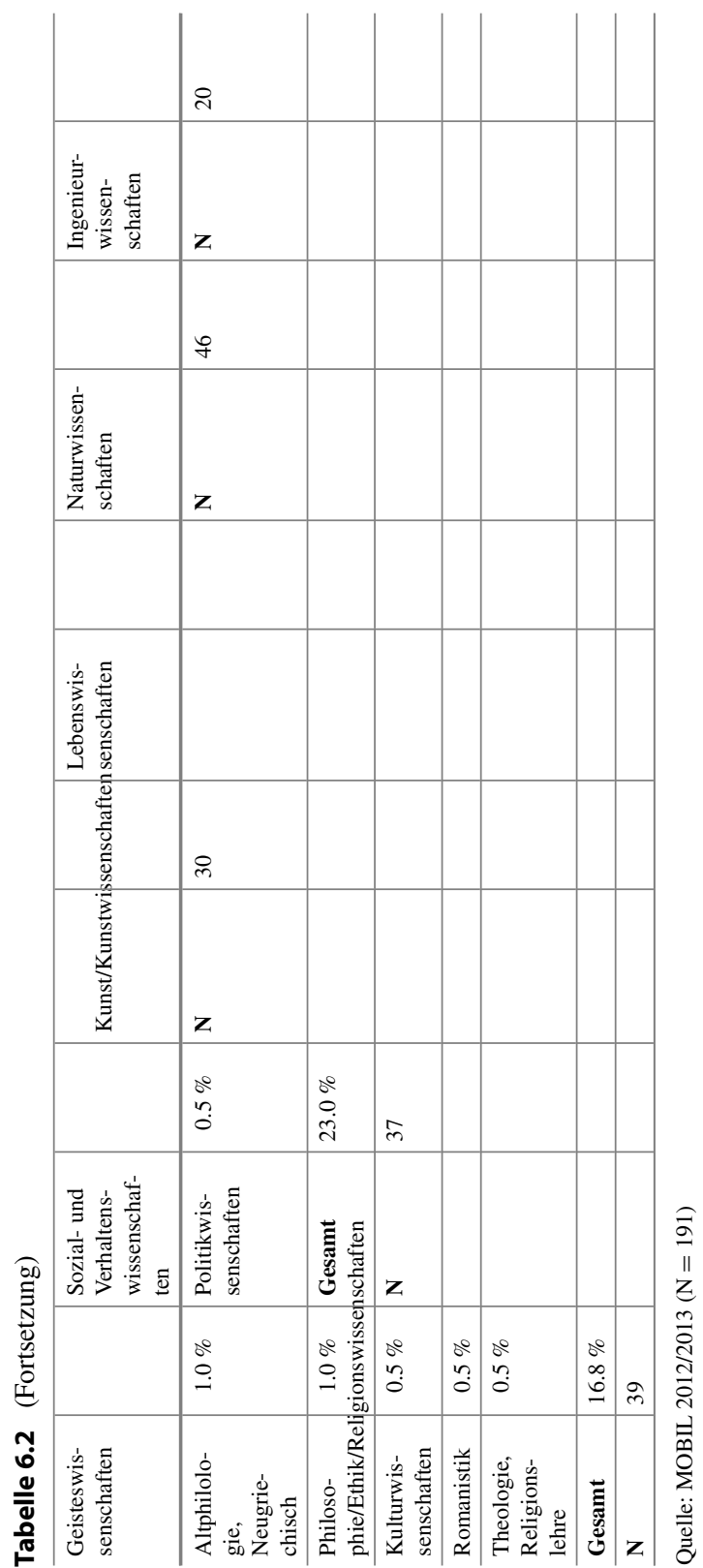


Aufgrund der Unterschiede hinsichtlich des angebotenen Fächerspektrums an Universitäten und Fachhochschulen erklären sich die deutlichen Divergenzen zwischen den Fächergruppen im Bereich der Fachhochschulprofessuren. Während die Geisteswissenschaften und die Lebenswissenschaften kaum an Fachhochschulen vertreten sind, liegt der Anteil in den Sozial-/Verhaltenswissenschaften (37 \%) und in den Ingenieurwissenschaften (45\%) hier in etwa doppelt so hoch wie unter allen Professoren mit Migrationshintergrund. Juniorprofessoren mit Migrationshintergrund arbeiten primär in den Geisteswissenschaften $(26 \%)$ und in den Sozial- und Verhaltenswissenschaften (23\%) sowie zum Teil in den Naturwissenschaften (15\%). Über die unterschiedlichen Anteile an Juniorprofessoren lassen sich auch Unterschiede in der Altersstruktur erklären. Das Durchschnittsalter variiert dabei zwischen 45 Jahren in den Sozial-/Verhaltenswissenschaften und 54 Jahren in den Ingenieurwissenschaften (vgl. Tabelle 6.3).

Die größten Unterschiede hinsichtlich der Fächergruppen zeigen sich erwartungsgemäß zwischen den Geschlechtern. Wie in der gesamten Professorenschaft arbeiten Professorinnen deutlich häufiger in den „Humanities“ als im Bereich „Science“. Allerdings zeigen sich auch innerhalb der Wissenschaftsbereiche durchaus erhebliche Unterschiede. Während Frauen über die Hälfte der Professoren in den Sozial- und Verhaltenswissenschaften ausmachen, liegt der Frauenanteil im Bereich Kunst/Kunstwissenschaften bei unter einem Drittel (vgl. Tabelle 6.3).

Tabelle 6.3 Fächergruppen nach Hochschulart und Geschlecht

\begin{tabular}{l|l|l|l|l|l}
\hline & $\mathrm{N}$ & Universität & Fachhochschule & Gesamt & Frauenanteile \\
\hline Naturwissenschaften & 46 & $25 \%$ & $21 \%$ & $24 \%$ & $24 \%$ \\
\hline Sozial-/Verhaltenswissenschaften & 44 & $18 \%$ & $40 \%$ & $23 \%$ & $52 \%$ \\
\hline Geisteswissenschaften & 32 & $21 \%$ & $2 \%$ & $17 \%$ & $41 \%$ \\
\hline Kunst/Kunstwissenschaften & 30 & $16 \%$ & $14 \%$ & $16 \%$ & $27 \%$ \\
\hline Ingenieurwissenschaften & 20 & $7 \%$ & $21 \%$ & $10 \%$ & $20 \%$ \\
\hline Lebenswissenschaften & 19 & $13 \%$ & $0 \%$ & $10 \%$ & $21 \%$ \\
\hline
\end{tabular}

Quelle: MOBIL 2012/2013 ( $N=191)$ Hochschulart x Fächergruppe: $p=0,021$ Hochschulart $x$ Geschlecht: $\mathrm{p}<0,000$; $\mathrm{p}$-value basiert auf $\chi^{2}$-Test

Hinsichtlich der Öffnung für Bewerber unterschiedlicher sozialer Herkunft zeigen sich die größten Unterschiede zwischen dem Bereich Kunst/Kunstwissenschaften und den Naturwissenschaften. Während in den 
Naturwissenschaften lediglich jeder fünfte Professor aus einem nichtakademischen Elternhaus stammt, beläuft sich der Anteil der Professoren der First Generation in der Kunst bzw. in den Kunstwissenschaften auf fast zwei Drittel (63\%). Zugleich liegen die Lebenswissenschaften (41\%) und die Ingenieurwissenschaften (39\%) leicht über und die Sozial-/Verhaltenswissenschaften (34\%) sowie die Geisteswissenschaften $(32 \%)$ in dieser Hinsicht leicht unter dem Durchschnitt.

Im Hinblick auf die verschiedenen Migrationstypen zeigt sich, dass die Hälfte der Professoren mit Migrationshintergrund in den Sozial/Verhaltenswissenschaften bereits in Deutschland geboren oder als Kind zugewandert ist. Demgegenüber stellen Professional Migrants über die Hälfte der Professoren der Geisteswissenschaften und Student Migrants über die Hälfte der Professoren der Kunst-/Kunstwissenschaften. Diese Zahlen können einen Hinweis auf unterschiedliche Rekrutierungspraktiken in den einzelnen Fächergruppen darstellen. Während die Sozial- und Verhaltenswissenschaften vergleichsweise offen erscheinen, insbesondere auch für Migranten der zweiten Generation, zeigt sich in den Geisteswissenschaften, dass hier in deutlich höherem Maße eine internationale Rekrutierung von erfahrenen Wissenschaftlern stattfindet. Im Bereich Kunst/Kunstwissenschaften durchlaufen Migranten besonders häufig eine akademische Karriere, wenn sie als internationale Studierende nach Deutschland gekommen sind. In den drei „Science“-Wissenschaftszweigen zeigen sich hingegen kaum nennenswerte Unterschiede bei der Verteilung der Migrationstypen.

Interessant sind zudem die unterschiedlichen Herkunftsregionen der Professoren in den jeweiligen Fächergruppen. Aufgrund der zum Teil geringen Fallzahlen sollten die Ergebnisse mit Vorsicht interpretiert werden. Dennoch ist es wichtig, folgende Beobachtungen festzuhalten. Die größte Diversität der Herkunftsregionen zeigt sich in den Sozial-/Verhaltenswissenschaften, den Naturwissenschaften und den Ingenieurwissenschaften. In allen drei Fächergruppen finden sich deutlich überdurchschnittlich hohe Anteile an Professoren aus Lateinamerika, Asien und Afrika. Eine Besonderheit der Ingenieurwissenschaften liegt zudem in einem enorm hohen Anteil osteuropäischer Professoren (40 \%). Demgegenüber dominieren in den Geisteswissenschaften, im Bereich Kunst/Kunstwissenschaften sowie in den Lebenswissenschaften Professoren aus Österreich, der Schweiz und weiteren westeuropäischen Ländern, die dort jeweils etwa $80 \%$ der Professoren mit Migrationshintergrund stellen. 


\subsection{Bildungs- und Berufsverläufe}

Das zweite Kapitel des Ergebnisteils stellt die Bildungs- und Berufsverläufe in den Mittelpunkt. Bei den Bildungsverläufen stehen die formalen Bildungsabschlüsse in unterschiedlichen Staaten im Mittelpunkt. Bei den Berufsverläufen werden die beruflichen Tätigkeiten und die unterschiedlichen Staaten der Berufstätigkeit vor der Berufung in den Fokus gerückt.

\subsubsection{Bildungsverläufe}

Die Bildungsverläufe werden auf zwei Ebenen dargestellt. Erstens wird eine differenzierte Betrachtung der Bildungsabschlüsse nach Zuwanderungszeitpunkt und beruflichen Merkmalen vorgenommen. Im zweiten Teil werden anhand der Staaten der Bildungsabschlüsse die Migrationswege der Professoren nachgezeichnet.

Eine abgeschlossene Promotion können $89 \%$ der befragten Professoren vorweisen. Der Anteil von $11 \%$ ohne Doktortitel lässt sich primär auf besondere Zugangsvoraussetzungen der Kunst/Kunstwissenschaften zurückführen, in denen zum Teil herausragende künstlerische Leistungen eine wichtigere Rolle spielen als wissenschaftliche Qualifikationen. So arbeiten von den Professoren ohne Promotionsabschluss allein $80 \%$ im Bereich Kunst/Kunstwissenschaften. Innerhalb dieser Disziplin liegt der Anteil an promovierten Professoren bei lediglich $36 \%$. Ebenfalls seltener promoviert sind Professoren in den Ingenieurwissenschaften $(18 \%)$ und an Fachhochschulen (20\%). Demgegenüber kann die jüngere Generation fast ausnahmslos eine abgeschlossene Promotion vorweisen $(98 \%)$, was darauf hindeutet, dass sowohl für Professoren in Deutschland als auch im Rahmen internationaler Rekrutierungsprozesse eine Berufung ohne abgeschlossene Dissertation - mit wenigen fachspezifischen Ausnahmen - de facto nicht mehr möglich ist.

Eine abgeschlossene Habilitation hat weniger als die Hälfte (47\%) der Professoren mit Migrationshintergrund. Nach der CAP-Befragung liegt der Habilitationsanteil in Deutschland bei 77 \% (Ates und Brechelmacher 2013). Ein wichtiger Erklärungsansatz für den geringen Anteil im Vergleich zu Professoren ohne Migrationshintergrund liegt darin, dass das Habilitationsmodell außerhalb von Deutschland kaum verbreitet ist. Folglich ist insbesondere bei der internationalen Rekrutierung von Professional Migrants davon auszugehen, dass Personen dieser Gruppe nur selten eine abgeschlossene Habilitation vorweisen können. Daher überrascht es zunächst, dass Professional Migrants mit $52 \%$ den höchsten Anteil an habilitierten Professoren stellen. Dieser hohe Anteil lässt sich zum 
einen damit erklären, dass ein Großteil der Professional Migrants aus Österreich und der Schweiz kommt, wo ebenfalls das Habilitationsmodell praktiziert wird. Ein weiterer wichtiger Erklärungsfaktor ist die Tatsache, dass Professional Migrants mit Abstand den geringsten Anteil an Fachhochschulprofessoren stellen. Universitätsprofessoren mit Migrationshintergrund haben zu $57 \%$ einen Habilitationsabschluss, während der Anteil an Fachhochschulen bei lediglich $13 \%$ liegt. Ein exklusiver Vergleich der Universitätsprofessoren zeigt, dass der Anteil hier unter Professional Migrants mit $55 \%$ etwas kleiner ausfällt als unter Early Migrants $56 \%$ und Student Migrants $62 \%$. Dennoch zeigt sich auch unter Kontrolle der Besoldungsgruppe und der Fächergruppen, dass Professional Migrants einen ähnlich hohen Anteil an habilitierten Professoren stellen.

Demgegenüber wurde die Juniorprofessur auch mit dem Argument eingeführt, dass sie möglicherweise perspektivisch die Habilitation als Qualifikationsphase ersetzen könne. Der Anteil habilitierter Juniorprofessoren mit Migrationshintergrund liegt bei $13 \%$. Auch hier zeigt sich, wie ebenfalls bereits in anderen Studien, dass ein Teil der Juniorprofessoren zusätzlich eine Habilitation anstrebt, um die Berufungschancen weiter $\mathrm{zu}$ verbessern (vgl. Abschnitt 2.2). Demgegenüber spielt die Habilitation für eine Berufung an Fachhochschulen nur eine untergeordnete Rolle (13\%). Im Rahmen eines Fächergruppenvergleichs zeigen sich die geringsten Anteile habilitierter Professoren im Bereich Kunst/Kunstwissenschaften (8\%), in den Sozial/Verhaltenswissenschaften (31\%) und in den Ingenieurwissenschaften (35\%), während Professoren in den Geisteswissenschaften (67 \%) sowie in den Lebenswissenschaften $(89 \%)$ deutlich häufiger eine Habilitation vorweisen können. Wie eingangs angedeutet lassen sich die Unterschiede zu einem großen Teil über die unterschiedliche Verteilung der Fächergruppen an Universitäten und Fachhochschulen erklären.

Im Folgenden werden die Migrationswege im Verlauf des Erwerbs der Bildungsabschlüsse zwischen den Bildungsabschlüssen näher in den Blick genommen. Professoren mit Migrationshintergrund sind zu $80 \%$ in einem anderen Staat als Deutschland geboren. Ein kleiner Teil dieser Gruppe (15\%) wandert bereits während der Kindheit nach Deutschland zu, wohingegen einige Professoren im Laufe der Kindheit in ein anderes Land als Deutschland migrieren. Die meisten Professoren allerdings (60\%) schließen zunächst in dem Land die Schule ab, in dem sie auch geboren sind. Nahezu alle Professoren, die in Deutschland geboren sind, schließen auch hier die Schule ab. Insgesamt wechselt jeder fünfte Professor zwischen der Geburt und dem Schulabschluss das Land (vgl. Abbildung 6.8).

Ein ähnlicher Migrationsverlauf zeigt sich zwischen dem Schulabschluss und dem ersten Studienabschluss. Hier erweist sich die Gruppe, die aus Deutschland 
ins Ausland migriert, als etwas größer. Von den $35 \%$, die in Deutschland die Schule abgeschlossen haben, erwerben $6 \%$ ihren ersten Studienabschluss im Ausland. Jeder zehnte Professor migriert zwischen Schulabschluss und erstem Studienabschluss nach Deutschland, während lediglich $3 \%$ zwischen zwei ausländischen Staaten migrieren. Über die Hälfte der Professoren erwirbt im selben ausländischen Staat den Studienabschluss, in dem auch die Schule abgeschlossen wurde (vgl. Abbildung 6.8).

Die anteilig größte Migration findet zwischen dem ersten und dem zweiten Studienabschluss statt. Dabei muss allerdings berücksichtigt werden, dass lediglich ca. ein Drittel der Professoren überhaupt einen zweiten Studienabschluss erwirbt. In dieser Gruppe findet bei über einem Drittel der Professoren (37 \%) während dieser Phase eine Migration zwischen zwei Nationalstaaten statt. Die größte Gruppe machen dabei Zuwanderer nach Deutschland (15\%) aus, wobei auch Migration zwischen ausländischen Staaten (14\%) hier eine wichtige Rolle spielt, während $8 \%$ aus Deutschland ins Ausland wechseln (vgl. Abbildung 6.8).

Deutlich geringer fällt hingegen die Migration zwischen dem Land des letzten Studienabschlusses und dem Land der Promotion aus. Lediglich jeweils $3 \%$ wechseln von Deutschland ins Ausland oder zwischen ausländischen Staaten. In etwa jeder elfte Professor wandert im Zeitraum zwischen diesen Abschlüssen nach Deutschland zu (vgl. Abbildung 6.8).

Wie bereits erwähnt muss hinsichtlich der Migration zwischen Promotion und Habilitation berücksichtigt werden, dass erstens die Habilitation außerhalb von Deutschland nur in wenigen weiteren Staaten erworben werden kann, und zweitens weniger als die Hälfte der Professoren überhaupt eine Habilitation abgeschlossen hat. Vor diesem Hintergrund erschließt sich, warum fast ein Viertel der Professoren aus dem Ausland für die Habilitation nach Deutschland wechselt. Über ein Drittel erwirbt die Habilitation allerdings auch im Ausland (vgl. Abbildung 6.9).

Generell stellt sich die Frage, in welchem Maße Professoren mit Migrationshintergrund in ihrem Bildungsverlauf international mobil waren. Grundlegend ist dabei auch zu ergründen, ob es viele Professoren gibt, die während ihrer Bildungslaufbahn gleich mehrfach international mobil waren, und wie hoch der Anteil an Professoren ausfällt, deren Bildungslaufbahn sich ausschließlich auf ein Land beschränkt. Zudem hat eine weitere Form von internationaler Mobilität, nämlich mögliche Auslandsaufenthalte während bestimmter Bildungsphasen wie beispielsweise im Rahmen eines studienbedingten Auslandssemesters, bisher keinerlei Berücksichtigung gefunden. Diese Fragen werden in Abschnitt 6.6.1 genauer in den Blick genommen. 
Der Anteil der in Deutschland erworbenen Bildungsabschlüsse unter Professoren mit Migrationshintergrund erhöht sich im Laufe des akademischen Werdegangs stetig von $35 \%$ (Schulabschluss) auf bis zu $63 \%$ bei der Habilitation. Die Staaten, in denen ausländische Bildungsabschlüsse erworben werden, ähneln erwartungsgemäß strukturell sehr stark den Herkunftsländern der Professoren. Am häufigsten wurden dabei Bildungsabschlüsse in Österreich, der Schweiz, den USA, den Niederlanden und dem Vereinigten Königreich erworben (vgl. Abbildung 6.9).

Auffällig ist generell, dass im Ausland erworbene Bildungsabschlüsse sich weitgehend auf höher entwickelte westliche Länder beschränken. Der Anteil an Bildungsabschlüssen aus Entwicklungs- und Schwellenländern variiert bei Schulund Studienabschlüssen zwischen $7 \%$ und $9 \%$, bei Promotionen liegt er sogar deutlich unter $5 \%$ (vgl. ausführlich Engel 2017).

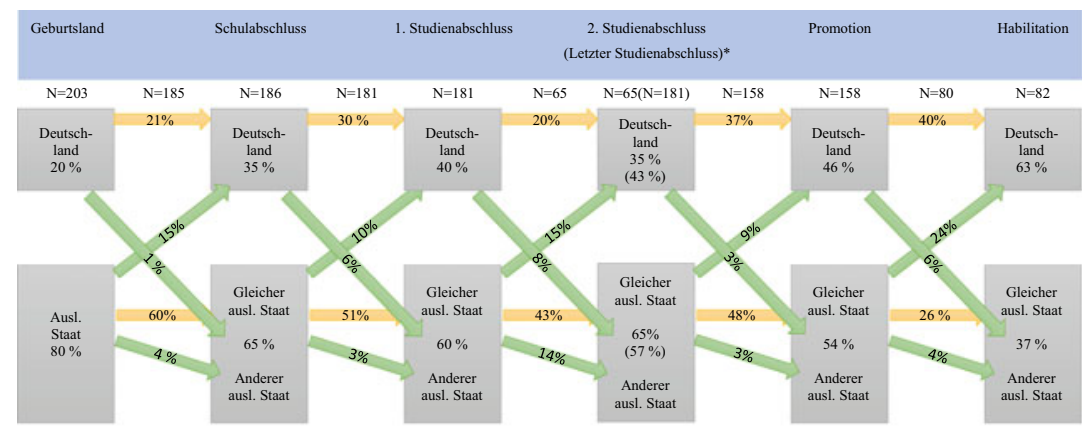

Kein Staatenwechsel

Staatenwechsel

Abbildung 6.8 Migrationswege zwischen den Bildungsabschlüssen (*Der letzte Studienabschluss bezieht sich den zweiten Studienabschluss bzw. bei Personen ohne zweiten Abschluss für den ersten Studienabschluss. In der folgenden Spalte werden Staatenwechsel zwischen dem letzten Studienabschluss und der Promotion betrachtet). (Quelle: MOBIL 2012/2013) 


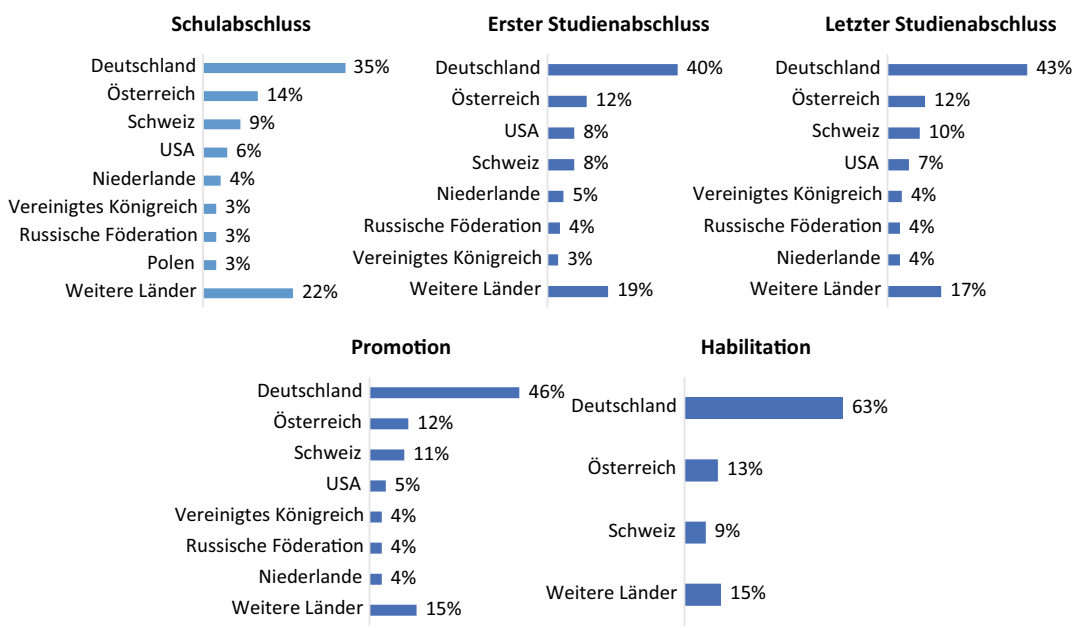

Abbildung 6.9 Staaten der Bildungsabschlüsse von Professoren mit Migrationshintergrund. (Quelle: MOBIL 2012/2013)

\subsubsection{Berufsverläufe}

Um die Berufsverläufe der Professoren mit Migrationshintergrund nachzuvollziehen, werden im Folgenden zunächst berufliche Tätigkeiten vor der Berufung auf die aktuelle Professur in den Blick genommen, bevor die Staaten der beruflichen Tätigkeiten näher betrachtet werden.

Knapp zwei Drittel sind bereits vor der Berufung einer wissenschaftlichen Tätigkeit an einer Hochschule nachgegangen. Etwas über die Hälfte konnte sich dabei über Stipendien finanzieren (vgl. Abbildung 6.10). Für deutlich über die Hälfte der Professoren (59 \%) stellt die aktuelle Professur an einer Hochschule in Berlin oder Hessen die Erstberufung dar. In etwa jeder vierte Professor gab hingegen an, zuvor bereits als Juniorprofessor, Vollprofessor oder Gastprofessor tätig gewesen zu sein. Andere berufliche Tätigkeiten außerhalb der Hochschule hat nach eigener Aussage knapp ein Drittel der Befragten ausgeübt (vgl. Abbildung 6.10). Erwartungsgemäß zeigen sich auch hier je nach Hochschulart deutliche Unterschiede. Während über $70 \%$ der Juniorprofessoren ihre wissenschaftliche Karriere zeitweise über ein Stipendium finanzieren konnten, liegt der entsprechende Anteil an Fachhochschulen lediglich bei ca. einem Drittel (35\%). Demgegenüber sind drei Viertel der Fachhochschulprofessoren anderen 
beruflichen Tätigkeiten außerhalb der Hochschule nachgegangen, während der Anteil an Universitäten in allen Besoldungsgruppen hier deutlich unter $20 \%$ liegt. Ähnliche Unterschiede zeigen sich bei wissenschaftlichen Tätigkeiten außerhalb der Hochschule zwischen Fachhochschulprofessoren (34 \%) und Universitätsprofessoren $(9 \%)$. Die beruflichen Tätigkeiten sowie die Unterschiede zwischen den Hochschularten zeigen sich in ähnlicher Form auch bei der CAP-Befragung der gesamten Professorenschaft in Deutschland (Höhle und Teichler 2016).

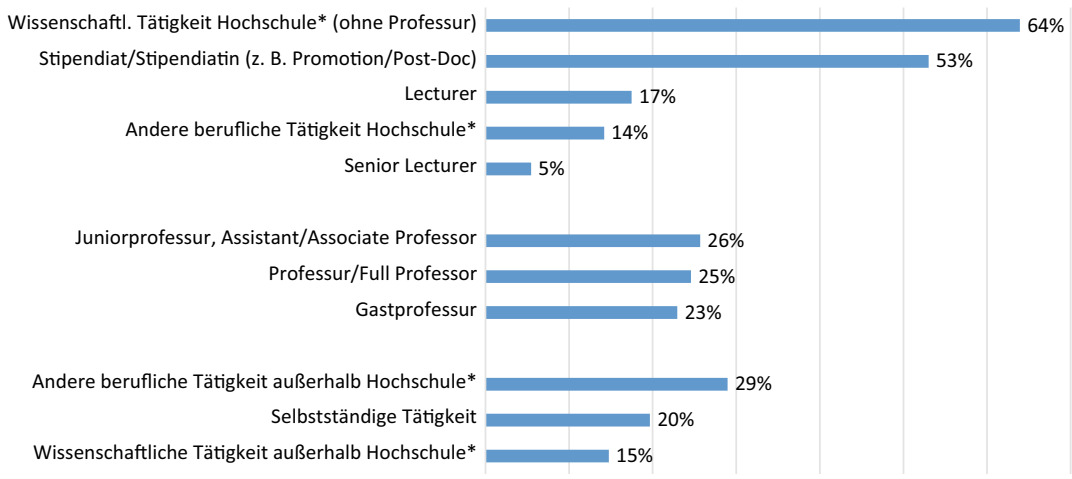

Abbildung 6.10 Berufliche Tätigkeiten vor der Berufung. (*Hochschule oder Forschungseinrichtung; Quelle: MOBIL 2012/2013 ( $\mathrm{N}=184$ ) [Mehrfachantworten])

Hinsichtlich des Ortes der beruflichen Tätigkeit zeigt sich zunächst, dass ca. vier Fünftel der Professoren bereits vor der Berufung einer Berufstätigkeit in Deutschland nachgegangen sind. Alle 203 Professoren zusammen haben in insgesamt 61 unterschiedlichen Staaten gearbeitet. Bei beruflichen Tätigkeiten im Ausland spielen insbesondere Tätigkeiten in den USA eine wichtige Rolle, wo bereits ein Drittel beruflich aktiv war. Dieser hohe Anteil legt den Schluss nahe, dass Berufserfahrungen in den USA sich für eine erfolgreiche Berufung als vorteilhaft erweisen. Dabei ist allerdings zu beachten, dass die Wahrscheinlichkeit, dass Professoren vor der Berufung in ihrem Herkunftsland beruflich aktiv waren, generell deutlich höher ist. Dementsprechend ist es wichtig, die Berufserfahrungen in anderen Ländern zum Anteil der Herkunftsländer in Relation zu setzen. Neben den USA weisen diesbezüglich insbesondere die Schweiz, das Vereinigte Königreich und Frankreich deutlich überdurchschnittliche Anteile auf (vgl. Abbildung 6.11 und Abbildung 6.5). 


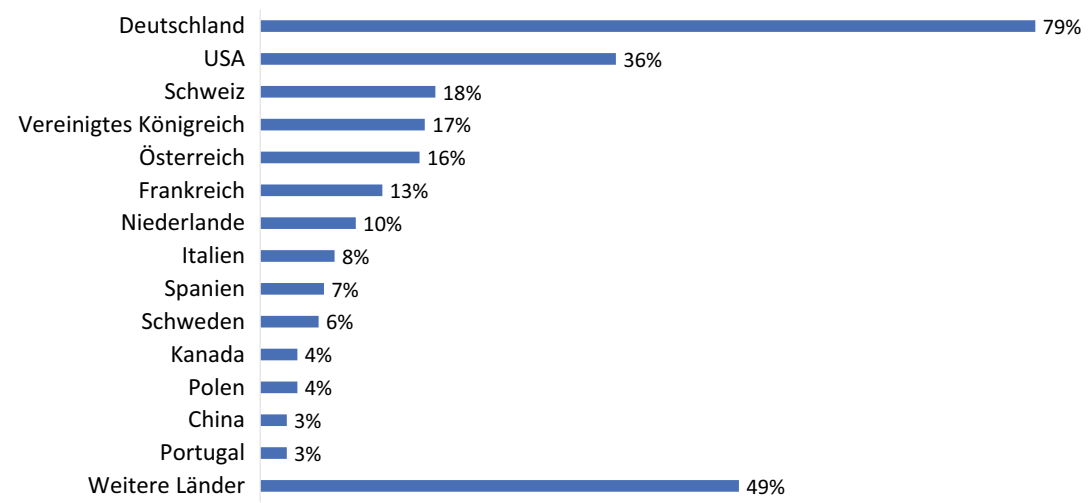

Abbildung 6.11 Staaten der beruflichen Tätigkeiten vor der Berufung. (Quelle: MOBIL 2012/2013 (N = 183) [Mehrfachantworten])

Die Frage, in welchem Maße Professoren mit Migrationshintergrund international mobil und dabei ggf. sogar in mehreren Staaten beruflich aktiv waren, wird detailliert in Abschnitt 6.6.1 in den Blick genommen. Abschließend ist der Berufungszeitpunkt detaillierter zu betrachten. Die Professoren wurden auf ihre aktuelle Professur allesamt zwischen 1989 und 2012, dem Jahr der Erhebung, berufen. Insgesamt $61 \%$ der Professoren wurden in den letzten sechs Jahren berufen und allein $26 \%$ im Laufe der letzten zwei Jahre vor der Erhebung. Dabei lassen sich drei Gruppen unterscheiden. Erstens ist die Gruppe der Juniorprofessoren, deren Vertragslaufzeit auf sechs Jahre festgeschrieben ist, zu nennen. Etwas über die Hälfte der Juniorprofessoren (54\%) wurde innerhalb der letzten drei Jahre berufen. Das durchschnittliche Berufungsalter liegt hier bei 34,2 Jahren. Zweitens sind die Erstberufungen auf Professuren „oberhalb“ der Juniorprofessur zu berücksichtigen. Für über die Hälfte dieser Gruppe stellt die Berufung auf die aktuelle Professur die Erstberufung dar. Hier liegt das Durchschnittsalter zum Zeitpunkt der Berufung bei 42,3 Jahren. Die dritte Gruppe besteht aus „Vollprofessoren“, die zuvor bereits eine andere Professur innehatten. In dieser Gruppe liegt das Berufungsalter im Durchschnitt bei 43,4 Jahren. Hinsichtlich des Alters zum Berufungszeitpunkt unterscheiden sich Professoren mit Migrationshintergrund nur geringfügig von der gesamten Professorenschaft. Nach dem Bundesbericht Wissenschaftlicher Nachwuchs liegt das Durchschnittsalter bei Juniorprofessoren bei 35,2, bei W2-Professoren bei 41,4 und bei W3-Professoren bei 42,4 Jahren (Autorengruppe BuWin 2017). 


\subsection{Beschreibung der Tätigkeitsbereiche, Drittmittel und Mitarbeiterzahl}

Das dritte Kapitel des Ergebnisteils gliedert sich in drei Teile. Zunächst geht es um die Verteilung der Arbeitszeit auf Forschung, Lehre und akademische Selbstverwaltung. Im Anschluss steht die Beurteilung der Professoren mit Migrationshintergrund über Ziele von Forschung und Lehre im Mittelpunkt, bevor die Mitgliedschaften und Funktionen an Hochschulen näher betrachtet werden. Der dritte Teil stellt die Mitarbeiterzahl und die damit eng verbundene Einwerbung von Drittmitteln in den Fokus der Analyse.

\subsubsection{Verhältnis von Forschung und Lehre}

Im Rahmen von Hochschullehrerbefragungen hat sich als ein zentraler Indikator zur Beschreibung der Arbeitssituation die Verteilung der Arbeitszeit auf Forschung, Lehre und akademische Selbstverwaltung etabliert. Da diese Verteilung zwischen den Semestern und der vorlesungsfreien Zeit stark variieren kann, wurde im Rahmen der MOBIL-Erhebung ausschließlich nach der Verteilung der Arbeitszeit während des Semesters gefragt. Demnach widmen Professoren mit Migrationshintergrund fast die Hälfte ihrer Arbeitszeit der Lehre inklusive Vorund Nachbereitung sowie Beratung und Betreuung der Studierenden. In etwa ein Drittel der Zeit wird für Forschung und Entwicklung sowie Projektmanagement verwendet und ca. ein Viertel der Zeit entfällt auf akademische Selbstverwaltung und sonstige Tätigkeiten. Diese Verteilung entspricht weitgehend der typischen Verteilung, die aus anderen Hochschullehrerbefragungen bekannt sind (Höhle und Teichler 2013b; Teichler 2017a; Teichler et al. 2017).

Ein weiterer Befund, der sich mit anderen Studien deckt, liegt darin, dass Fachhochschulprofessoren auch in Anbetracht des deutlich höheren Lehrdeputats von 18 Semesterwochenstunden im Vergleich zu 8-10 Semesterwochenstunden bei W2- und W3-Professoren einen größeren Teil ihrer Zeit für die Lehre verwenden. Juniorprofessoren haben mit vier Semesterwochenstunden - bzw. sechs Semesterwochenstunden nach der Evaluation - das vergleichsweise geringste Lehrdeputat, wodurch sich auch der relativ geringe Zeitanteil der Lehre erklären lässt. Zugleich handelt es sich dabei um die Professorengruppe, in der im Vergleich die meiste Zeit der Forschung gewidmet wird. W3-/C4-Professoren verweisen ebenfalls auf einen vergleichsweisen geringen Anteil der Lehre an der Gesamtarbeitszeit. Allerdings nimmt für diese Gruppe die akademische Selbstverwaltung deutlich mehr Zeit in Anspruch. Die Zeit für Forschung fällt bei Professoren aus den 
Lebenswissenschaften und den Naturwissenschaften gegenüber Professoren anderer Fachbereiche mit Abstand am höchsten aus, wobei hier wiederum der geringe Anteil dieser Fächergruppen an Fachhochschulen berücksichtigt werden muss (vgl. Abbildung 6.12). Hinsichtlich der verschiedenen Altersgruppen verwendet die jüngere Generation den größten Anteil der Zeit für die Forschung, was sich primär über das geringere Lehrdeputat der Juniorprofessoren erklären lässt. Zwischen den Geschlechtern zeigen sich indessen nur geringfügige Unterschiede.

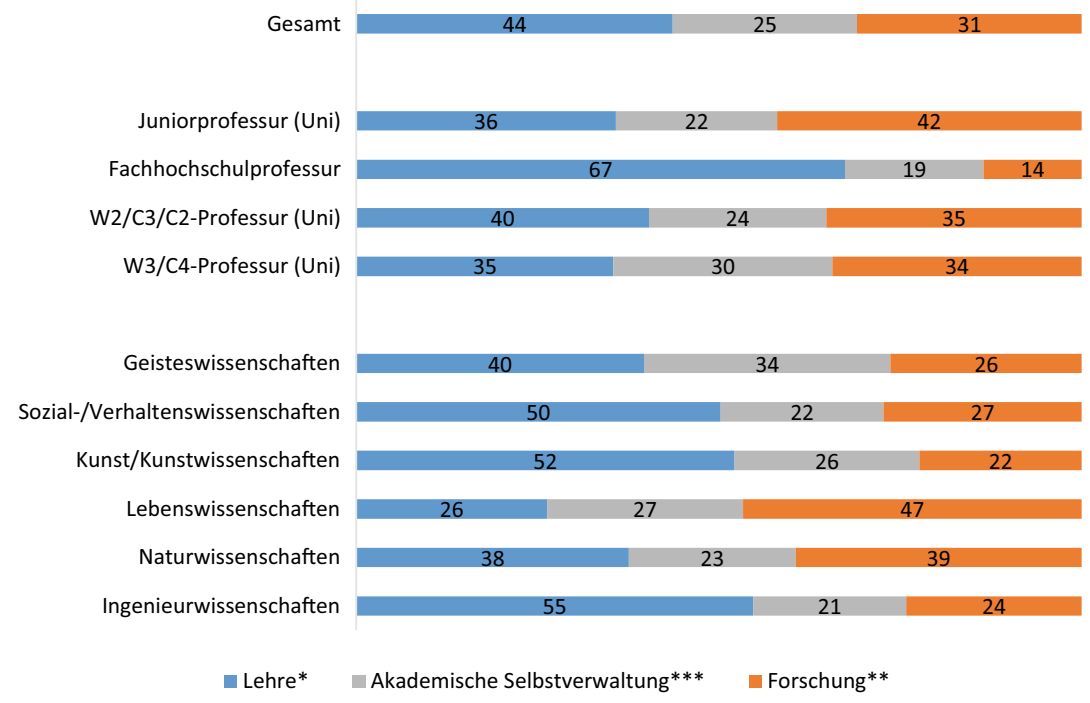

Abbildung 6.12 Verhältnis der Arbeitszeit für Lehre, Forschung und Selbstverwaltung (*(inkl. Vor- und Nachbereitung, Beratung und Betreuung) ** Forschung und Entwicklung (inkl. Projektmanagement) ***Akademische Selbstverwaltung und sonstige Tätigkeiten). (Quelle: MOBIL 2012/2013 (Gesamt: N = 194, Besoldungsgruppen N = 194, Lehre, Akademische Selbstverwaltung, Forschung $\mathrm{p}<0,000$, Fächergruppen $\mathrm{N}=191$, Lehre, Akademische Selbstverwaltung, Forschung $\mathrm{p}<0,000)$; $\mathrm{p}$-value basiert auf einfaktorieller ANOVA)

Fast die Hälfte der Professoren mit Migrationshintergrund betont, dass ihre Präferenz im Bereich der Forschung liege, während lediglich ca. jeder siebte Befragte nach eigener Aussage den Tätigkeitsbereich Lehre präferiert. Im Altersvergleich zeigt sich, dass über die Hälfte der jüngeren Generation ihre Präferenz sowohl in der Forschung als auch in der Lehre sieht, also in dieser Frage keine eindeutige Präferenz äußern kann. In den mittleren und älteren Generationen nimmt 
der Anteil derer, die eine Präferenz für beide Bereiche äußern, zunehmend ab. Gleichzeitig lässt sich hier eine Polarisierung erkennen, im Rahmen derer von einer zunehmend großen Gruppe jeweils eine eindeutige Präferenz für den Bereich Lehre oder für den Bereich Forschung geäußert wird. Inwieweit dieser Befund auf unterschiedliche generationsspezifische Einstellungen zurückzuführen ist oder ob möglicherweise mit zunehmender Berufserfahrung tendenziell eine stärkere Positionierung hinsichtlich der eigenen Tätigkeitsbereiche stattfindet, lässt sich auf dieser Grundlage nicht abschließend beurteilen (vgl. Abbildung 6.13).

Auffällig ist zudem, dass der Anteil derer, die eine Präferenz für den Tätigkeitsbereich Lehre äußern, unter Juniorprofessoren besonders gering und an Fachhochschulen hingegen mit Abstand am höchsten ausfällt. Der hohe Anteil unter Fachhochschulprofessoren ist aufgrund des allgemein bekannten hohen Lehrdeputats wenig überraschend, da sich vermuten lässt, dass insbesondere Personen mit hoher Affinität für die Lehre diese Positionen anstreben. Dennoch fällt auf, dass selbst in der Gruppe der Fachhochschulprofessoren der Anteil der Personen höher ist, die den Bereich Forschung bevorzugen. Zwischen „Science“ und „Humanities“ zeigen sich ähnliche Unterschiede wie zwischen den Generationen. Während in etwa die Hälfte der ,Science “-Professoren keinen der beiden Bereiche eindeutig präferiert, positionieren sich Professoren in den „Humanities “ deutlich stärker entweder in der Forschung oder in der Lehre (vgl. Abbildung 6.13).

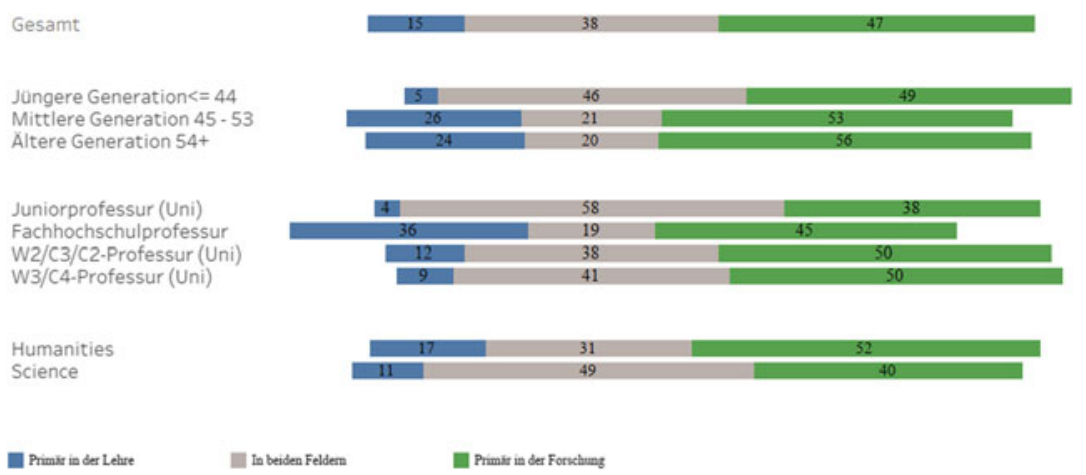

Abbildung 6.13 Unterschiede bei den Präferenzen hinsichtlich Lehre und Forschung. (Quelle: MOBIL 2012/2013 (Gesamt: $\mathrm{N}=197$, Alter: $\mathrm{N}=196$ ( $\mathrm{p}=0,01)$, Besoldungsgruppe: $\mathrm{N}=192(\mathrm{p}<0,01)$, Fächergruppen: $\mathrm{N}=189(\mathrm{p}=0,03)$. $\mathrm{p}$-value basiert auf $\chi^{2}$-Test $)$ 
Zusammenfassend lässt sich festhalten, dass, wenngleich Professoren mit Migrationshintergrund im Durchschnitt mehr Zeit für Lehre aufwenden, die generelle Präferenz doch deutlich stärker im Bereich Forschung liegt. Goastellec und Pekari (2013a) zeigen anhand der CAP Befragung, dass Professorinnen in Deutschland Forschung noch deutlich stärker gegenüber der Lehre präferieren als ihre männlichen Kollegen, de facto aber einen deutlich höheren Anteil der Zeit der Lehre widmen. Bei den Professoren mit Migrationshintergrund zeigen sich weder bei der Zeitgestaltung noch bei den Präferenzen nennenswerte Unterschiede zwischen den Geschlechtern.

\subsubsection{Mitgliedschaften und Funktionen innerhalb und außerhalb der Hochschulen}

Neben Forschung und Lehre kommt der akademischen Selbstverwaltung eine wichtige Bedeutung zu. Vor diesem Hintergrund ist es wenig überraschend, dass über $80 \%$ der Professoren bereits in Hochschulgremien aktiv waren. Dabei übernehmen sie häufig auch Führungs- bzw. Leitungsfunktionen. Fast die Hälfte der Befragten hatte bereits die Leitung eines Instituts inne und über ein Viertel war bereits als (Studien-)Dekan oder Prodekan tätig. Zudem war in etwa jeder sechste Professor mit Migrationshintergrund bereits Beauftragter für Internationalisierung. Etwas über die Hälfte verwies zudem auf weitere Aktivitäten. Die Leitung oder Koordination von Studiengängen oder Graduiertenschulen wurde dabei mit Abstand am häufigsten genannt (vgl. Abbildung 6.14). Weitere häufig wahrgenommene Funktionen sind die Mitgliedschaft in Berufungskommissionen, der Vorsitz des Prüfungsausschusses sowie die Tätigkeit als Frauenbeauftragte.

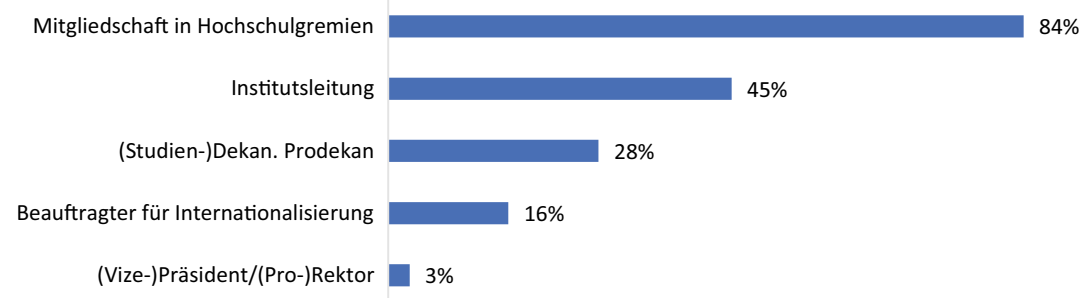

Abbildung 6.14 Wahrnehmung von Funktionen innerhalb der Hochschule. (Quelle: MOBIL 2012/2013 ( $=176$ ) [Mehrfachantworten]) 
Neben den Tätigkeiten innerhalb der Hochschule war Gegenstand der Erhebung auch die Frage, in welchen Feldern Professoren mit Migrationshintergrund außerhalb der Hochschule aktiv sind. Über drei Viertel der Befragten sind nach eigener Aussage Mitglied in Fach- oder Wissensgesellschaften. Auch Tätigkeiten für Institutionen der Forschungsförderung üben fast zwei Drittel der Professoren mit Migrationshintergrund aus. Demgegenüber sind sie deutlich seltener in Gewerkschaften/Berufsverbänden, in der wissenschaftlichen Politikberatung oder in Hochschulräten/-kuratorien aktiv (vgl. Abbildung 6.15).

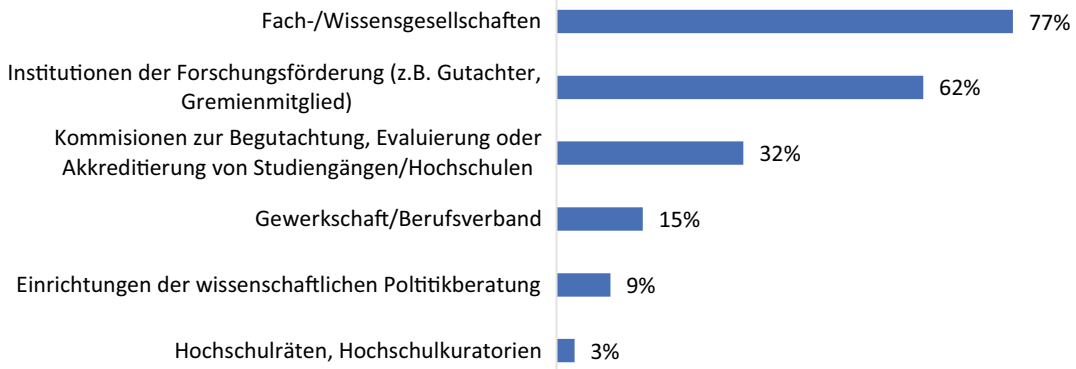

Abbildung 6.15 Berufliche Aktivitäten und Mitgliedschaften außerhalb der Hochschule. (Quelle: MOBIL 2012/2013 (N = 164) [Mehrfachantworten])

Wie auch in anderen Hochschullehrerbefragungen (vgl. Jacob und Teichler 2011: S. 163-165) zeigt sich, dass Universitätsprofessoren in diesem Feld aktiver sind als ihre Kollegen an Fachhochschulen. Während Universitätsprofessoren mit Migrationshintergrund zu $83 \%$ Mitglieder in Fach- und Wissensgesellschaften und zu $69 \%$ in Institutionen der Forschungsförderung aktiv sind, liegen die entsprechenden Anteile an Fachhochschulen lediglich bei $57 \%$ bzw. $37 \%$.

\subsubsection{Mitarbeiterzahl und eingeworbene Drittmittel}

Im Folgenden wird zum einen untersucht, welche Zahl an Wissenschaftlichen, Studentischen und Verwaltungsmitarbeitern Professoren mit Migrationshintergrund zur Verfügung steht. Zum anderen wird beleuchtet, inwieweit und in welcher Höhe durch Professoren mit Migrationshintergrund erfolgreich Drittmittel eingeworben wurden. Der Zusammenhang der beiden Fragen liegt darin, 
dass der Schluss naheliegt, dass insbesondere die Zahl der Wissenschaftlichen und Studentischen Mitarbeiter positiv mit der Zahl eingeworbener Drittmittelprojekte korreliert. Neben den eingeworbenen Drittmitteln sind auch Besoldungsund Fächergruppe für die Zahl der Mitarbeiter von zentraler Relevanz. Zu beachten ist hier, dass nicht zwischen Voll- und Teilzeitstellen differenziert werden konnte. Ein Vergleich über sogenannte Vollzeitäquivalente wäre indessen noch aussagekräftiger. Generell lassen sich eingeworbene Drittmittel und die damit zusammenhängende Mitarbeiterzahl als ein zentraler Indikator der Leistungsmessung im deutschen Hochschulwesen beschreiben.

Professoren mit Migrationshintergrund sind durchschnittlich in etwa fünf Wissenschaftliche Mitarbeiter zugeordnet. Hier ist darauf hinzuweisen, dass es eine Reihe von Professoren mit Migrationshintergrund gibt, deren Mitarbeiterzahl um ein Vielfaches höher liegt. Aufgrund dieser Ausreißer nach oben erscheint es sinnvoll, anstelle des arithmetischen Mittels den Median als Maß der zentralen Tendenz heranzuziehen. Der Median liegt bei drei Wissenschaftlichen Mitarbeitern (vgl. Abbildung 6.16). Eine Differenzierung nach Besoldungsgruppen gibt Aufschluss über die genauere Verteilung. An Fachhochschulen gibt es traditionell

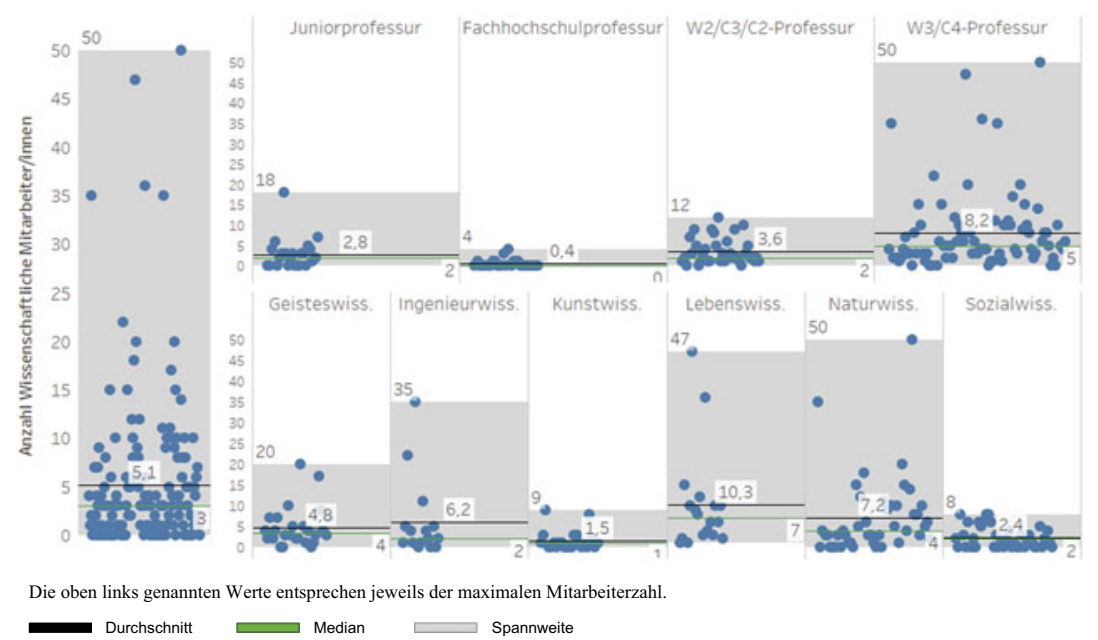

Abbildung 6.16 Wissenschaftliche Mitarbeiter nach Besoldungsgruppe und Fächergruppe. (Quelle: MOBIL 2012/2013: Gesamt $N=175$, Besoldungsgruppe $N=170$ ( $p<0,001)$, Fächergruppe $\mathrm{N}=169(\mathrm{p}=0,001)$; $\mathrm{p}$-value basiert auf einfaktorieller ANOVA) 
nur einen kleinen wissenschaftlichen Mittelbau und auch der Anteil eingeworbener Drittmittel fällt hier im Vergleich zu den Universitäten deutlich geringer aus, was die Tatsache erklärt, dass Fachhochschulprofessoren mit Migrationshintergrund nur in Ausnahmefällen über einzelne Wissenschaftliche Mitarbeiter verfügen. Etwas höher fällt der Anteil bei Juniorprofessoren und W2-/C3-/C2Professoren aus, für die der Median bei einer Zahl von zwei Wissenschaftlichen Mitarbeitern liegt. Mit Abstand am höchsten liegt der Median bei W3-/C4 Professoren (fünf Wissenschaftliche Mitarbeiter), denen auf der Basis des arithmetischen Mittels sogar ca. acht Wissenschaftliche Mitarbeiter zugeordnet sind. Auffällige Unterschiede sind ebenso zwischen den Fächergruppen zu beobachten. Insbesondere Professoren aus den Lebenswissenschaften und den Ingenieurwissenschaften verfügen über eine große Zahl Wissenschaftlicher Mitarbeiter, während im Bereich Kunst/Kunstwissenschaften und in den Geisteswissenschaften die Zahlen eher gering ausfallen (vgl. Abbildung 6.16).

Die Zahl der Studentischen Mitarbeiter von Professoren mit Migrationshintergrund liegt durchschnittlich bei 3,6 bzw. bei einem Median von 2. Die Gruppenunterschiede entsprechen in weiten Teilen der Verteilung bei den Wissenschaftlichen Mitarbeitern. Generell fällt die Zahl der Studentischen Mitarbeiter im Vergleich zu den Wissenschaftlichen Mitarbeitern an Universitäten etwas geringer aus. Eine Ausnahme bilden Fachhochschulprofessoren, die im Durchschnitt zumindest eine Studentische Mitarbeiterkraft beschäftigen können (vgl. Abbildung 6.17).

Hinsichtlich der Mitarbeiter im Bereich Technik, Service und Verwaltung (MTSV) liegt der Median bei einem Mitarbeiter. Dabei ist zu beachten, dass Professoren an Fachhochschulen und Juniorprofessoren nur in Ausnahmefällen eine MTSV-Stelle zugeordnet ist. W2-/C3-/C2-Professoren können durchschnittlich über eine Person im Bereich MTSV verfügen. Der Median bei W3-/C4Professuren liegt ebenfalls nur bei einem Mitarbeiter. Hier gibt es allerdings eine beträchtliche Zahl von Ausreißern, insbesondere in den Lebenswissenschaften und den Naturwissenschaften, in denen allgemein eine große Zahl von Mitarbeitern aus dem Bereich Technik, Service und Verwaltung zu finden ist (vgl. Abbildung 6.18).

Beachtliche Unterschiede zeigen sich zwischen Professoren mit und ohne Drittmittelprojekte/n. Ein Vergleich der beiden „Extremgruppen“, Professoren ohne Drittmittelprojekte einerseits und Professoren mit einer durchschnittlichen Jahressumme von über $500.000 €$ anderseits, bringt für alle drei Mitarbeitergruppen große Unterschiede zum Vorschein. Die durchschnittliche Zahl Wissenschaftlicher Mitarbeiter variiert zwischen 1,1 und 14,5. Bei den Studentischen 


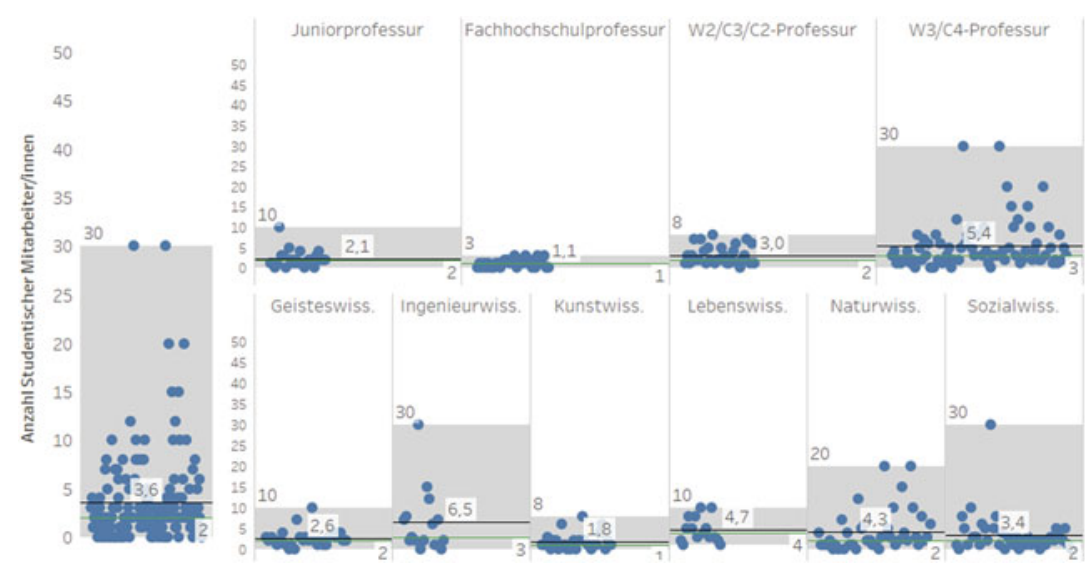

Die oben links genannten Werte entsprechen jeweils der maximalen Mitarbeiterzahl.

Durchschnitt

Median

$\square$ Spannweite

Abbildung 6.17 Studentische Mitarbeiter nach Besoldungsgruppe und Fächergruppe. (Quelle: MOBIL 2012/2013: Gesamt N = 173 Besoldungsgruppe $N=168(p<0,001)$, Fächergruppe $\mathrm{N}=168(\mathrm{p}=0,023)$-p-value basiert auf einfaktorieller ANOVA)

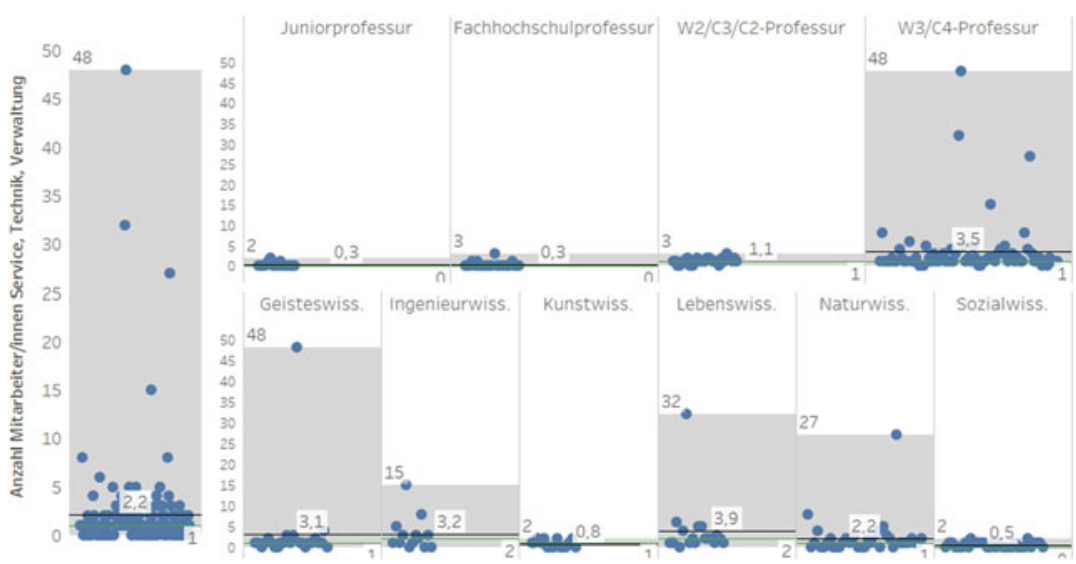

Die oben links genannten Werte entsprechen jeweils der maximalen Mitarbeiterzahl.

Durchschnitt $\square$ Median Spannweite

Abbildung 6.18 MTSV nach Besoldungsgruppe und Fächergruppe. (Quelle: MOBIL 2012/2013: Gesamt $N=147$ Besoldungsgruppe $N=142(p=0,02)$ Fächergruppe $N=$ 137 ( $\mathrm{p}=0,199)$; - $\mathrm{p}$-va lue basiert auf einfaktorieller ANOVA) 
Mitarbeitern liegen die entsprechenden Werte bei 1,3 und 8,0 und im Bereich der MTSV bei 0,5 und 7,0.

Hinsichtlich der Einwerbung von Drittmitteln sollte im Rahmen der Erhebung ermittelt werden, inwieweit und in welcher Höhe Professoren mit Migrationshintergrund in den letzten Jahren in Deutschland Drittmittel eingeworben haben. Dabei stellt sich zunächst die Frage, inwieweit Professoren, die erst in den letzten ein bis zwei Jahren nach Deutschland gekommen sind, bei der Analyse berücksichtigt werden sollten, da sich hier argumentieren ließe, dass ein Professor nur ein vergleichsweise kleines Zeitfenster zur Einwerbung von Drittmitteln zur Verfügung hatte. Im Rahmen eines Vergleichs wird allerdings sichtbar, dass diese Gruppe von Professoren nicht seltener oder in geringerem Maße Drittmittel eingeworben hat als Professoren, die bereits länger in Deutschland arbeiten. Aufgrund dessen kann die folgende Analyse sich auf das gesamte Sample beziehen.

Knapp die Hälfte der Professoren mit Migrationshintergrund wirbt keine oder ausschließlich kleine Drittmittelprojekte mit einer durchschnittlichen Jahressumme von weniger als $50.000 €$ ein. Größere Drittmittelprojekte mit einem Jahresbudget von über $150.000 €$ wirbt mehr als ein Drittel der Professoren ein. Die mit Abstand größten Unterschiede zeigen sich je nach Besoldungsgruppe. Fast $90 \%$ der Fachhochschulprofessoren werben keine oder nur in geringem Maße Drittmittel ein. Die Gründe hierfür liegen, wie eingangs erläutert, in erster Linie in der strategischen Ausrichtung dieser Hochschulart, die traditionell stärker Lehre und Praxisbezug in den Mittelpunkt stellt. Mehr als ein Viertel der Juniorprofessoren und ca. ein Drittel der W2-/C3-/C2-Professoren an Universitäten wirbt hingegen größere Drittmittelprojekte ein. Mit Abstand am erfolgreichsten im Bereich Drittmittelakquise sind W3-/C4-Professoren, was sicher unter anderem auf die deutlich bessere Personal- und Sachmittelausstattung zurückzuführen ist. Aus dieser Besoldungsgruppe wirbt ca. die Hälfte der Professoren mit Migrationshintergrund größere Drittmittelprojekte ein (vgl. Abbildung 6.19).

Deutliche Unterschiede werden auch zwischen den Fächergruppen sichtbar. Während im Bereich Kunst/Kunstwissenschaften über $70 \%$ der Professoren und in den Sozial-/Verhaltenswissenschaften ca. $50 \%$ keine oder lediglich kleine Drittmittelprojekt einwerben, haben fast $80 \%$ der Professoren in den Naturwissenschaften Drittmittel mit einer durchschnittlichen Jahressumme von mindestens $50.000 €$ eingeworben. Auch in den Ingenieurwissenschaften fällt der Anteil höher aus als bei den Kollegen. Zwischen den Geschlechtern zeigen sich kaum Unterschiede. Die Gruppenunterschiede stellen sich in ähnlicher Form dar als bei der gesamten Professorenschaft in Deutschland. Der von Böhmer et al. (2011) beobachtete Unterschied nach Alterskohorten, dass Professoren zwischen 40 und 


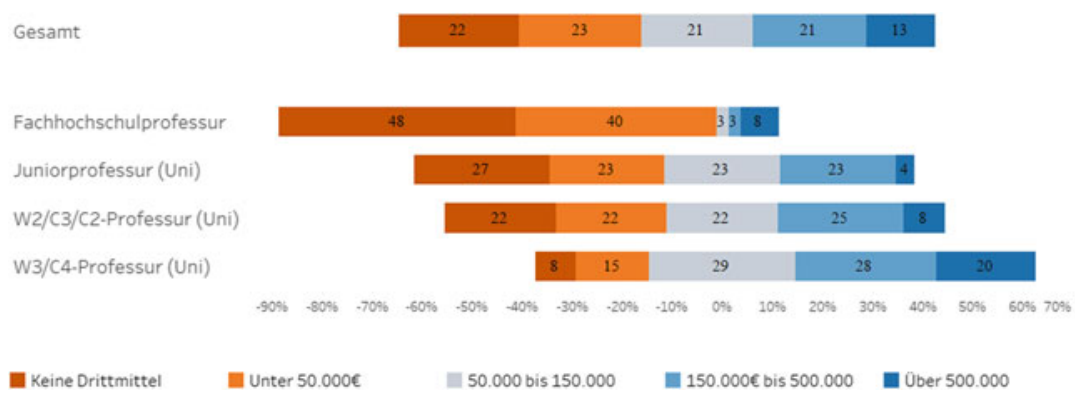

Abbildung 6.19 Drittmittel nach Besoldungsgruppe. (Quelle: MOBIL 2012/2013 (N = $179(\mathrm{p}<0,001))$; $\mathrm{p}$-value basiert auf Kruskal-Wallis-Test)

55 Jahren deutlich häufiger und höhere Drittmittel als ihre Kollegen, zeigt sich bei Professoren mit Migrationshintergrund nicht.

Im Übrigen zeigen sich signifikante Gruppenunterschiede auch zwischen den verschiedenen Migrationstypen. Professional Migrants liegen in Bezug auf Drittmittelakquise nicht nur vor den Early Migrants, sondern auch mit deutlichem Abstand vor den Student Migrants. Die Unterschiede lassen sich primär über die Verteilung nach Hochschularten und Fächergruppen erklären. Professional Migrants sind fast ausschließlich an Universitäten tätig. Demgegenüber machen Student Migrants erstens einen überdurchschnittlich hohen Anteil an Fachhochschulprofessoren aus. Zweitens ist jeder vierte Student Migrant im Bereich Kunst/Kunstwissenschaften beschäftigt und damit in der Fächergruppe, die mit Abstand den geringsten Drittmittelanteil stellt.

\subsection{Berufsverständnis und Beurteilung von Hochschulreformen/-diskursen}

Im folgenden Kapitel geht es darum, wie Professoren mit Migrationshintergrund sich zu bestimmten Zielen in Forschung und Lehre sowie zu hochschulpolitischen Diskursen und Reformen positionieren. Ein Großteil der Professoren mit Migrationshintergrund konnte im Bildungs-/Berufsverlauf umfangreiche Erfahrungen in Hochschulsystemen anderer Länder machen. Dementsprechend können 
sie in gewisser Weise ${ }^{15}$ eine Außenperspektive oder zumindest eine ,andere“ Perspektive auf das Hochschulwesen einnehmen. Die folgenden Analysen können vor diesem Hintergrund interessante Einblicke liefern, einerseits wie sich über die Positionierung zu den genannten Aspekten unterschiedliche Berufsverständnisse zeigen und wie die Professoren aus ihrer Perspektive bestimmte Diskurse und Reformen im Hochschulwesen in Deutschland beurteilen. Ausgehend von der Beurteilung unterschiedlicher Ziele von Forschung und Lehre (Abschnitt 6.4.1), werden anschließend Standpunkte zu Bologna-Reformen (Abschnitt 6.4.2) sowie zum Diskurs und den Reformen des New Public Managements (Abschnitt 6.4.3) näher beschrieben. Abschließend werden die Beurteilungen zur „Öffnung der Hochschule“ (Abschnitt 6.4.4) und zur vertikalen Differenzierung des Hochschulwesens (Abschnitt 6.4.5) herausgearbeitet.

\subsubsection{Beurteilung der Ziele von Forschung und Lehre}

Zunächst stellt sich die Frage, wie Professoren mit Migrationshintergrund Forschungs- und Lehrbedingungen an der Hochschule beurteilen und welche Bedeutung die beiden Bereiche für die eigene Motivation und das Selbstverständnis haben.

Die Erzeugung neuen Wissens durch Forschung sehen fast zwei Drittel der Professoren als die zentrale Aufgabe der Hochschulen (vgl. Abbildung 6.20). In dieser Hinsicht zeigen sich deutliche Unterschiede insbesondere zwischen Professoren an Fachhochschulen, von denen weniger als die Hälfte dieser Aussage zustimmt, und W3-/C4-Professoren sowie Juniorprofessoren, von denen jeweils fast drei Viertel diese Ansicht teilen (vgl. Abbildung 6.20).

Die wissenschaftliche Anerkennung in der Fachgemeinschaft stellt für fast die Hälfte der Professoren die wichtigste Motivation dar. Dieser Befund deutet ebenfalls auf einen stärkeren Fokus der Professorenschaft im Bereich Forschung hin, da bisher die Anerkennung in der Fachgemeinschaft zu großen Teilen auf erbrachten Forschungsleistungen basiert. Zugleich wird sichtbar, dass diese Anerkennung insbesondere für die jüngere Generation von großer Relevanz ist, von der über die Hälfte der Aussage zustimmt (vgl. Abbildung 6.20). Auch hier zeigt sich, dass diese Ansicht an Fachhochschulen in deutlich geringerem Maße geteilt wird

\footnotetext{
${ }^{15}$ Wie stark die Befragten selbst davon sprechen würden eine Außenperspektive einzunehmen, worin diese für sie besteht und welchen Einfluss u. a. Herkunftsland und Zuwanderungszeitpunkt darauf haben, stellen wichtige Fragestellungen für zukünftige Studien dar. Erste interessante Erkenntnisse liefern hierzu auch die Analysen zum Selbstverständnis der Professoren (vgl. Abschnitt 6.10).
} 
als an Universitäten. Darüber hinaus zeigen sich große Unterschiede zwischen den Fächergruppen. Während in den Naturwissenschaften die Anerkennung in der Fachgemeinschaft für über $60 \%$ der Professoren die wichtigste Motivation darstellt, stimmt aus dem Bereich Kunst/Kunstwissenschaft lediglich ein Viertel dieser Aussage zu. Zudem teilen über die Hälfte der Professional Migrants diese Ansicht, wobei hier der hohe Anteil an Universitätsprofessoren als zentraler Erklärungsansatz zu berücksichtigen ist.

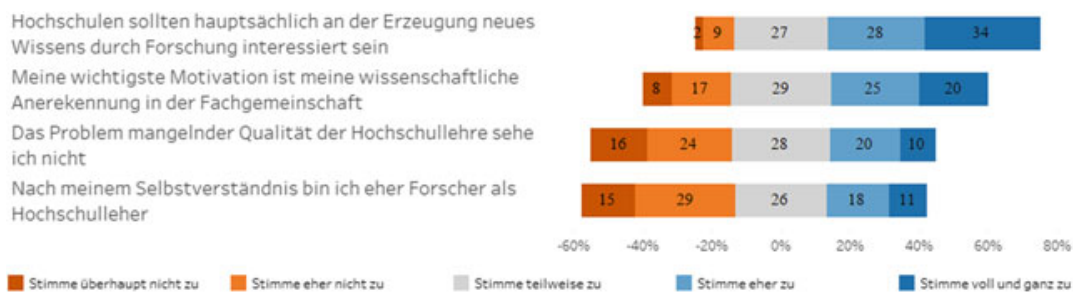

Abbildung 6.20 Einstellung zu Forschung und Lehre. (Quelle: MOBIL 2012/2013 (Item 1: $\mathrm{N}=178$ Item 2: $\mathrm{N}=178$ Item 3: $\mathrm{N}=176$ Item 4: $\mathrm{N}=178$ ))

Eine häufig geäußerte Kritik am Hochschulwesen liegt darin, dass durch die starke Fokussierung auf Forschung aufgrund deren Relevanz für eine erfolgreiche Laufbahn und Reputation die Qualität der Hochschullehre leide. Eine derart kritische Perspektive auf die Hochschullehre nimmt auch die Mehrheit der Professoren mit Migrationshintergrund ein. Während knapp ein Drittel kein Problem bei der Qualität der Hochschullehre sieht, äußern über $40 \%$ Kritik an der allgemeinen Lehrqualität an Hochschulen (vgl. Abbildung 6.20). Dabei ist interessant, dass insbesondere Juniorprofessoren die Qualität der Lehre in Frage stellen. Über zwei Drittel dieser Gruppe äußern entsprechende Kritik.

Hinsichtlich des Selbstverständnisses nehmen sich ca. $30 \%$ der Professoren selbst weniger als Hochschullehrer, sondern primär als Forscher wahr (vgl. Abbildung 6.20). Erwartungsgemäß trifft dies in deutlich geringerem Maße auf Fachhochschulprofessoren zu. Auffällige Unterschiede zeigen sich auch zwischen den Fächergruppen. Während jeweils über ein Drittel in den Lebenswissenschaften, den Naturwissenschaften und den Sozial-/Verhaltenswissenschaften der Aussage zustimmt, liegen die Zustimmungswerte im Bereich Kunst/Kunstwissenschaften und in den Geisteswissenschaften bei unter einem Viertel. In den Ingenieurwissenschaften stimmen sogar lediglich $6 \%$ dieser Aussage zu. 


\subsubsection{Beurteilung der Bologna-Reformen}

Hinsichtlich der Beurteilung der Hochschulentwicklung und der Hochschulreformen werden im Folgenden Veränderungen im Zuge der Bologna-Reformen näher betrachtet. Die Hochschulentwicklung in Deutschland in den letzten zwei Jahrzehnten war in besonderem Maße durch Reformen im Zuge des Bologna-Prozesses geprägt. Die Bologna-Reformen umfassen dabei eine Vielzahl unterschiedlicher Zielsetzungen. Im Mittelpunkt steht dabei insbesondere die Vereinheitlichung von Studiengängen und -abschlüssen und die Stärkung der internationalen Mobilität mit dem Ziel, einen einheitlichen europäischen Hochschulraum zu schaffen. Zugleich spielen Qualitätssicherung und die Etablierung des Konzepts des Lebenslangen Lernens an Hochschulen eine wichtige Rolle (Wolter 2012). Insbesondere in Deutschland war die Einführung der Bachelorund Masterstudiengänge zudem mit der Hoffnung verbunden, hierdurch eine Verkürzung der Studiendauer zu erreichen (Döbert 2013).

Drei zentrale Zielsetzungen im Zuge der Bologna-Reformen sind im Folgenden näher zu betrachten: die Förderung internationaler Mobilität, die Einführung gestufter Studiengänge und die Verkürzung der Studiendauer. Die einheitliche Umstellung auf das Bachelor-Master-Modell für den Großteil der Studiengänge im europäischen Hochschulraum wurde primär damit begründet, dass sich auf diese Weise internationale Mobilität und internationale Mobilitätsprogramme leichter realisieren lassen. Diese Zielsetzung bewerten $60 \%$ der Professoren mit Migrationshintergrund positiv (vgl. 6 21). Die Einführung neuer Studienmodelle führte in Deutschland auch dazu, dass eine Vielzahl von Diplom- und Magisterstudiengängen durch ein zweistufiges System von Bachelor- und Masterstudiengängen ersetzt wurden. Die Vor- und Nachteile dieser Entwicklung werden unter Professoren deutlich kontroverser diskutiert. Während 34 \% der Professoren die Entwicklung positiv bewerten, ist die Zahl derer, die der Umstellung kritisch gegenüberstehen, sogar geringfügig größer (vgl. Abbildung 6.21).

Ein weiterer Aspekt der Umstellung bestand darin, die Regelstudienzeit von sechs Jahren bei Magister- und Diplomstudiengängen auf fünf Jahre im Bachelor-Master-System zu verkürzen. Ein häufig geäußerter Kritikpunkt in diesem Zusammenhang lautete, dass die Umstellung weniger eine Neukonzipierung als vielmehr eine Komprimierung der Studieninhalte zur Folge habe. Auch darin könnte ein Grund dafür liegen, dass diese Zielsetzung von Professoren mit Migrationshintergrund am kritischsten beurteilt wird. Insgesamt bewerten über $40 \%$ der Professoren diese Zielsetzung negativ (vgl. Abbildung 6.21).

Während die positive Bewertung internationaler Mobilitätsprogramme in allen Gruppen relativ breite Zustimmung erfährt, zeigen sich bei der Einführung 


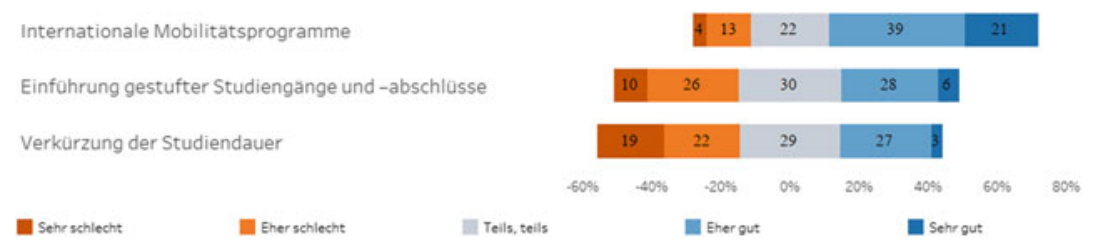

Abbildung 6.21 Beurteilung der Zielsetzungen der Bologna-Reformen. (Quelle: MOBIL 2012/2013 (Item 1: $N=183$ Item 2: $N=178$ Item 3: $N=181$ ))

gestufter Studiengänge bzw. -abschlüsse und der Verkürzung der Studiendauer deutliche Unterschiede je nach Herkunftsregion und Zuwanderungsalter. So beurteilen Early Migrants beide Zielsetzungen deutlich kritischer als Professoren, die erst während des Studiums oder der Berufstätigkeit nach Deutschland gekommen sind. Zudem werden die beiden Zielsetzungen insbesondere von Professoren aus Osteuropa sowie aus Österreich und der Schweiz negativ bewertet, während Professoren aus angelsächsischen Ländern diese mit Abstand am positivsten beurteilen. Ein Grund hierfür könnte darin liegen, dass in den angelsächsischen Ländern Bachelor-Master-Strukturen schon seit langer Zeit etabliert sind und die Umstellungen Austausch und Kooperationen mit Deutschland erleichtert haben.

\subsubsection{Standpunkte zum NPM-Diskurs und zu konkreten NPM-Reformen}

Wenngleich die Bologna-Reformen eine Vielzahl von Zielsetzungen verfolgten, spielen Hochschulsteuerung und Hochschulgovernance auf den ersten Blick keine zentrale Rolle. Dennoch zeigen sich durchaus Berührungspunkte insbesondere in den Feldern Qualitätssicherung, Evaluationen und Akkreditierungen. Die Umsetzung der Bologna-Reformen war zu einem hohen Grad auf Managementstrukturen und Hochschulsteuerung angewiesen (Wolter 2012). Infolgedessen lassen sich Wolter zufolge trotz aller nationalen Besonderheiten folgende fünf Trends der Hochschulentwicklung in Europa beobachten (vgl. Wolter 2012: S. 131-133):

1. Die Reduzierung detaillierter staatlicher Kontrolle und die Erweiterung der institutionellen Autonomie

2. Stärkung des institutionellen Managements und Professionalisierung des Hochschulmanagements

3. Qualitätssicherung, Akkreditierung und öffentliche Rechenschaftspflicht 
4. Zunehmende Betonung des Wettbewerbs und marktähnlicher Mechanismen

5. Neue Finanzierungs- und Zuteilungsverfahren

Der Diskurs über New Public Management (NPM) in Deutschland kreist speziell um die Frage, wie Entscheidungsstrukturen, Personalpolitik, Leistungsmessung und -steuerung effizienter gestaltet werden können, indem Konzepte speziell aus dem Dienstleistungssektor privatwirtschaftlicher Unternehmen aufgegriffen werden. Insoweit lassen sich unterschiedliche Kernbereiche ausmachen, in denen das Governance-Modell des NPM sich vom traditionellen Modell deutscher Universitäten unterscheidet. Das traditionelle Modell basiert insbesondere auf den beiden Pfeilern der staatlichen Regulierung und Detailsteuerung sowie der akademischen Selbstverwaltung. Das NPM-Modell zielt hingegen darauf ab, den Einfluss dieser beiden Institutionen deutlich $\mathrm{zu}$ verringern und im Gegenzug eine umfassendere Außensteuerung und interne Hierarchie sowie einen stärkeren Wettbewerb zu etablieren (Hüther und Krücken 2016).

Zusammenfassend lässt sich das deutsche Hochschulwesen nach gegenwärtigem Stand als ein hybrides Modell charakterisieren, in dem eine Verflechtung von Bestandteilen des traditionellen Modells mit neuen NPM-Elementen stattfindet. Für die Professoren ist speziell der Aspekt der akademischen Selbstverwaltung von Bedeutung. Hier zeigt sich, dass, auch wenn sich im Zuge der NPMReformen ein nicht unbedeutender Wandel verzeichnen lässt, die grundlegenden Entscheidungsbefugnisse weiterhin in der Hand der akademischen Profession und nicht bei Administratoren oder Managern liegen (Krücken et al. 2013; Schimank und Lange 2009).

Im Rahmen der vorliegenden Arbeit wird die Einstellung der Professoren mit Migrationshintergrund zum New Public Management auf zwei Ebenen untersucht. Erstens soll untersucht werden, wie sie sich generell zum New Public Management Diskurs positionieren. Diesbezüglich werden Fragen zur Anwendungsorientierung von Forschung, zur Informationspflicht gegenüber der Öffentlichkeit und zur Dienstleistungsausrichtung an Hochschulen aufgeworfen. Im zweiten Schritt geht es dann um die Beurteilung konkreter Reformmaßnahmen im Rahmen des NPM Ansatzes wie Lehrevaluationen, Globalhaushalt, der Stärkung des Hochschulmanagements, Zielvereinbarungen und der Akkreditierung von Studiengängen.

Die stärkere Anwendungsorientierung der Forschung ist ein Thema, das unter Wissenschaftlern die Gemüter spaltet und den NPM-Diskurs begleitet. Die Forderung nach höherer Effizienz des NPM-Ansatzes impliziert zugleich die Forderung nach Verwertbarkeit und Praxisbezug. Zugleich betrifft es insbesondere die Frage nach dem Verhältnis zwischen Grundlagenforschung und 
anwendungsorientierter Forschung. Fast die Hälfte der Professoren mit Migrationshintergrund betont die Relevanz der Anwendungsorientierung. Demgegenüber spielt für ca. ein Viertel dieses Thema keine wichtige Rolle (vgl. Abbildung 6.22). Die Relevanz des Anwendungsbezuges wird von Professorinnen deutlich stärker hervorgehoben. Erwartungsgemäß zeigen sich auch hier Unterschiede zwischen Universitäten und Fachhochschulen. Fachhochschulen sind traditionell stärker auf anwendungsorientierte Forschung ausgerichtet, worin ein wichtiger Grund dafür liegen könnte, dass über zwei Drittel der Fachhochschulprofessoren mit Migrationshintergrund der Anwendungsorientierung einen hohen Stellenwert beimessen. Ebenfalls von zentraler Bedeutung ist die Differenzierung nach Fächergruppen. Während über der Hälfte der Professoren aus den Ingenieurwissenschaften, den Sozial-/Verhaltenswissenschaften und den Naturwissenschaften die Anwendungsorientierung als wichtig bezeichnen, liegt der entsprechende Anteil in den Geisteswissenschaften bei lediglich $14 \%$ (vgl. Abbildung 6.23).

Neben der Anwendungsorientierung wird häufig auch die geringe Öffentlichkeitsarbeit von Professoren kritisch hinterfragt. Eine entsprechende Informationspflicht lässt sich insbesondere daraus herleiten, dass das staatliche Hochschulwesen eine steuerfinanzierte Institution darstellt. In Verbindung mit dem NPM-Ansatz steht hier die Frage des unmittelbaren gesellschaftlichen Nutzens im Mittelpunkt. Über $40 \%$ der Professoren mit Migrationshintergrund sind der Meinung, dass Hochschulen ihrer Informationspflicht gegenüber der Öffentlichkeit in ausreichendem Maße nachkommen, während fast ein Viertel der Professoren diese Auffassung nicht teilt (vgl. Abbildung 6.22). Interessant ist dabei, dass insbesondere die ältere Generation ab 54 Jahren der Öffentlichkeitsarbeit kritisch gegenübersteht. Während lediglich ca. ein Fünftel der jüngeren und mittleren Generation von einer zu geringen Öffentlichkeitsarbeit ausgeht, beläuft sich der entsprechende Anteil in der älteren Generation auf fast ein Drittel. Auch Professoren, die bereits sehr lange (über 20 Jahre) oder bereits in zweiter Generation in Deutschland leben, haben einen deutlich kritischeren Blick auf die Umsetzung der Informationspflicht.

Die Reformen und der Diskurs über eine umfassende New-PublicManagement-Agenda an Hochschulen lassen sich vereinfacht in der Frage zuspitzen, inwieweit sich Hochschulen zu modernen Dienstleistungunternehmen entwickeln sollten. Es geht dabei um die Frage, inwieweit eine stärker wettbewerbsorientierte Steuerung und Ressourcenverteilung an Hochschulen stattfinden soll, die häufig mit einer höheren Effizienz und Qualitätssicherung begründet werden (Wolter 2012).Die deutliche Mehrheit (60\%) steht einer solchen Entwicklung ablehnend gegenüber, während mehr als ein Drittel sich sogar entschieden gegen 

Die Anwendungsorientierung der Forschung ist mir
wichtig

Hochschulen nehmen ihre Informationspflicht gegenuber der Offentlichkeit in ausrreichendem Maße wahr

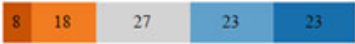

\begin{tabular}{|l|l|l|l|}
23 & 35 & 36 & 5 \\
\hline
\end{tabular}

Hochschulen sollten sich zu modernen Dienstleistungsunternhmen entwicklen

a stimme uberhaupt nicht zu

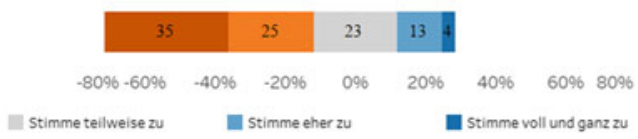

Abbildung 6.22 Einstellung zu ausgewählten Aspekten des NPM-Diskurses. (Quelle: MOBIL 2012/2013 (Item 1: $\mathrm{N}=175$, Item 2: $\mathrm{N}=168$, Item 3: $\mathrm{N}=179$ ))

eine solche Entwicklung ausspricht. Dennoch würde auch ca. jeder sechste Professor eine solche Entwicklung eher positiv bewerten (vgl. Abbildung 6.22). Eine besonders kritische Haltung zeigt sich im Bereich Kunst/Kunstwissenschaften sowie in den Natur- und Geisteswissenschaften (vgl. Abbildung 6.23).

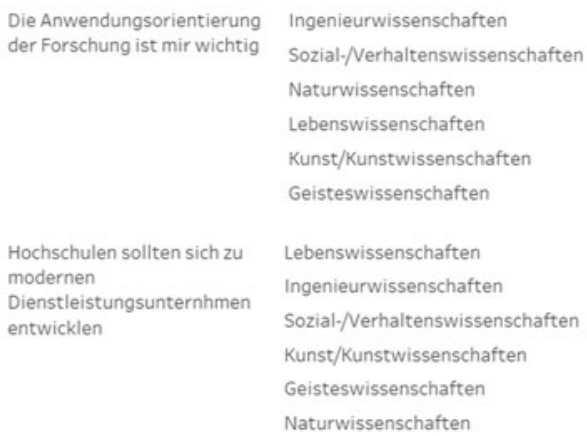

Ingenieurwissenschaften

Sozial-/Verhaltenswissenschaften

Naturwissenschaften

Lebenswissenschaften

Kunst/Kunstwissenschaften

Geisteswissenschaften

Lebenswissenschaften

Ingenieurwissenschaften

Sozial-/Nerhaltenswissenschaften

Kunst/Kunstwissenschaften

Geisteswissenschaften

Naturwissenschaften

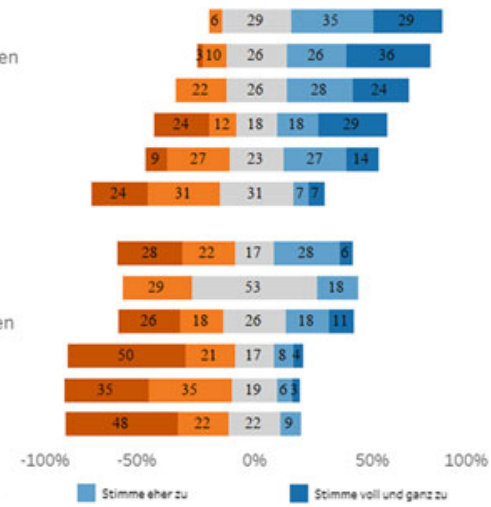

Abbildung 6.23 Einstellung zu ausgewählten Aspekten des NPM-Diskurses nach Fächergruppen. (Quelle: MOBIL 2012/2013 (Item 1: $N=170(p=0,009)$, Item 2: $N=174(p=$ 0,083)); p-value basiert auf Kruskal-Wallis-Test)

Die dargestellten Analysen liefern erste Anhaltspunkte zur Beantwortung der Frage, wie sich Professoren mit Migrationshintergrund zum New-PublicManagement-Diskurs an Hochschulen positionieren. In einem zweiten Schritt 
wird detailliert betrachtet, wie sie konkrete Maßnahmen bewerten, die bereits in den letzten Jahren in unterschiedlichem Maße an Hochschulen etabliert wurden. Konkret handelt es sich um die nachfolgenden fünf Reformen: Stärkung des Hochschulmanagements (Präsidium/Dekanate), Globalhaushalt, Zielvereinbarungen in der Hochschule, Lehrevaluation und Akkreditierung von Studiengängen/Qualitätssicherung.

Darüber, dass Lehrevaluation ein wichtiges Mittel darstellen kann, um die Qualität von Lehrveranstaltungen zu untersuchen und zu verbessern, besteht weitgehend Konsens unter Wissenschaftlern an Hochschulen. Deutlich kontroverser wird hingegen die Frage diskutiert, inwieweit die zurzeit gängige Praxis, Lehrveranstaltungen von Studierenden über standardisierte Fragebögen evaluieren zu lassen, in dieser Hinsicht zielführend ist. Kritisch angemerkt wird dabei, dass es eine Vielzahl von Faktoren gebe, die - unabhängig von der Qualität der Lehrveranstaltung - einen wichtigen Einfluss auf die Evaluation haben können. Dazu zählen die Zusammensetzung der Studierenden, Unterschiede zwischen Wahlund Pflichtveranstaltungen sowie das damit verbundene Vorinteresse am Thema, der Einfluss der eigenen Benotung auf die Beurteilung und schließlich die Tatsache, dass Studierende, die die Lehrveranstaltung vorzeitig abbrechen, an der am Veranstaltungsende stattfindenden Evaluation in der Regel nicht mehr teilnehmen. Ein weiterer Kritikpunkt besteht darin, dass der Prozess der Evaluation sowohl für die Evaluierten als auch für die Gutachter viel Zeit in Anspruch nimmt, die dadurch für Forschung und Lehre nicht mehr zur Verfügung steht (Schimank und Lange 2009, Wollbring 2013). Insgesamt wird der Indikator Lehrevaluation von den fünf Indikatoren jedoch mit Abstand am positivsten bewertet. Deutlich über die Hälfte der Professoren spricht sich hierfür aus (vgl. Abbildung 6.24). Mit Abstand am kritischsten stehen Professoren aus dem Bereich Kunst/Kunstwissenschaft Lehrevaluationen gegenüber. Diese werden dort mehrheitlich negativ bewertet. Demgegenüber beurteilen drei Viertel der Professoren aus den Sozial-/Verhaltenswissenschaften Lehrevaluationen positiv.

Der Wandel von einer zentralistisch ausgerichteten Hochschulfinanzierung hin zu einem Globalhaushalt geht damit einher, dass Hochschulleitungen deutlich mehr Spielraum bei der Ausgestaltung ihres Haushalts haben. Die Entscheidungsmacht darüber, wie öffentliche Mittel in der Hochschule verteilt werden, liegt deutlich stärker als zuvor in der Hand der Hochschulen. Zugleich behält sich allerdings auch die Politik über die Kopplung von Zielvorgaben an die Höhe des Globalbudgets weiterhin Einflussmöglichkeiten vor (Schimank und Lange 2009, Wollbring 2013). Dementsprechend lässt sich der Wandel hin zum Globalhaushalt nicht ausschließlich als Stärkung der Autonomie, sondern eher als eine veränderte Form von Hochschulgovernance beschreiben. Der Großteil der Professoren mit 
Migrationshintergrund (40\%) bewertet diesen Wandel positiv, während ca. ein Viertel diese Entwicklung negativ beurteilt (vgl. Abbildung 6.24).

Ein weiteres Ziel der NPM-Maßnahmen liegt in der Stärkung des Hochschulmanagements. Nach Wolter (2012) geht es dabei insbesondere um die Entscheidungsbefugnisse der Leitung sowohl auf der Hochschul- als auch auf Fakultätsebene, die Verlagerung von Einflussmöglichkeiten auf der Ebene der akademischen Selbstverwaltung hin zur Managementebene, neue Steuerungselemente wie Zielvereinbarungen und leistungsorientierte Mittelvergabe und darum, Auswahlprozesse in der Wissenschaft stärker durch Institutionen wie externe Gremien oder recruitment agencies zu gestalten. Bei dem Wandel ist $\mathrm{zu}$ berücksichtigen, dass auch die Managementebene an Hochschulen primär durch Personen mit wissenschaftlichen Biographien geprägt ist. Professoren mit Migrationshintergrund sind in dieser Frage gespalten. Die Gruppe der Befürworter ist mit einem Anteil von $36 \%$ etwas größer als die Gruppe der Kritiker (vgl. Abbildung 6.24). Bei den Befürwortern handelt es sich insbesondere um Professoren aus Osteuropa und den angelsächsischen Ländern. Professoren anderer Herkunftsregionen stehen einer solchen Stärkung des Hochschulmanagements in der Mehrheit ablehnend gegenüber.

Zielvereinbarungen zwischen Hochschulleitungen und Professoren stellen ebenfalls ein neues Mittel im Zuge der NPM-Reformen dar. Durch die Koppelung der Zielvereinbarung an die Gewährung von Leistungsbezügen wird ein verstärkter personeller Kontrollmechanismus geschaffen (Hüther und Krücken 2016, S. 185). In etwa ein Drittel der Professoren bewertet derartige Zielvereinbarungen negativ. Etwas größer ist die Gruppe der Befürworter. Insgesamt sprechen sich $37 \%$ dafür aus, dass zusätzliche Leistungsbezüge für die Einhaltung von Zielvereinbarungen gewährt werden können (vgl. Abbildung 6.24). Interessant ist dabei insbesondere, dass ein nicht unerheblicher Teil der jüngeren Generation (50\%) dieses stärker indikatoren- und leistungsbasierte Verfahren positiv bewertet.

Ein zentrales Element der NPM-Reformen ist es, Prozesse der Qualitätssicherung stärker im Hochschulwesen zu verankern. Auch im Kontext der Umstellung auf das Bachelor-Master-Modell wurde ein Akkreditierungssystem im deutschen Hochschulwesen etabliert. Im Bereich der Lehre überprüfen unabhängige, externe Agenturen in bestimmten Abständen die ,Qualität“ von Studiengängen. Ein Mittel im Wege dieses „Qualitätssicherungsverfahrens“ stellen beispielsweise Absolventenbefragungen der jeweiligen Studiengänge dar, um zu prüfen, in welchem Maße das Ziel der Employability dort erreicht wurde. Durch das neu geschaffene Akkreditierungssystem wird dem alten System der akademischen Selbstverwaltung, das ausschließlich auf internen Entscheidungsprozessen basierte, ein externes Kontrollgremium gegenübergestellt (Wolter 2012, Hüther und Krücken 2016, S. 219). 
Professoren mit Migrationshintergrund beurteilen diesen Aspekt des NPM mit Abstand am negativsten (41 \%). Dennoch beurteilt auch ca. ein Drittel die Einführung positiv (vgl. Abbildung 6.24). Zudem fällt auf, dass in besonderem Maße männliche Professoren sowie W3-/C4-Professoren die Akkreditierung negativ beurteilen.

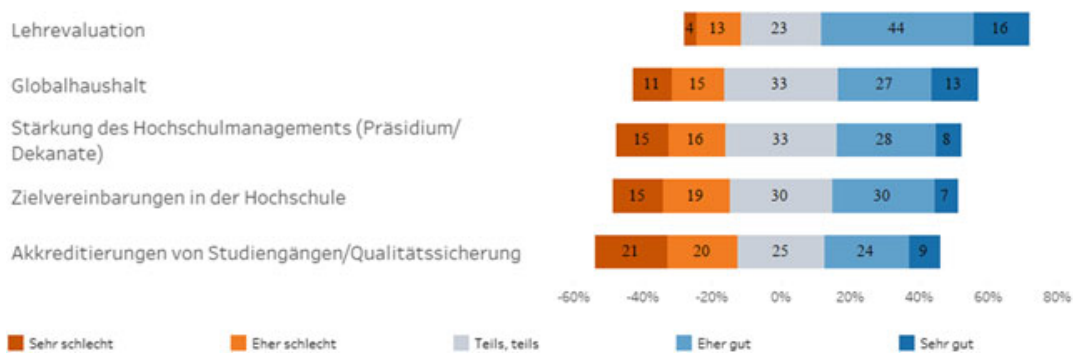

Abbildung 6.24 Einstellung $\mathrm{zu}$ ausgewählten NPM-Reformen. (Quelle: MOBIL 2012/2013 (Item 1: $N=186$ Item 2: $N=150$ Item 3: $N=172$ Item 4: $N=165$ Item $5: \mathrm{N}=177)$ )

Abschließend soll mithilfe der Skalenbildung untersucht werden, wie Professoren mit Migrationshintergrund NPM-Reformen insgesamt beurteilen. Dazu wird zunächst eine Faktorenanalyse vorgeschaltet. Bei der Faktorenanalyse werden über eine Korrelationsmatrix sogenannte Faktoren extrahiert. Ziel ist es, die Eindimensionalität der ausgewählten Items zu prüfen (Kuckartz et al. 2013, S. 246).

Für die fünf Items hinsichtlich der NPM-Reformen wurde eine Hauptkomponentenanalyse mithilfe von SPSS Statistics 25 durchgeführt. Zunächst werden die Voraussetzungen zur Durchführung einer Faktorenanalyse geprüft. Die Korrelationsmatrix basiert fast durchgehend auf Koeffizienten, die über 0,3 liegen. Der Kaiser-Meyer-Olkin-Wert als Maß der Strichprobeneignung liegt bei 0,75 und damit über dem geforderten Mindestwert von 0,6. Der Bartlett-Test ist ebenfalls statistisch signifikant. Die Hauptkomponentenanalyse bestätigt die Eindimensionalität der fünf Items. Es gibt lediglich eine Komponente mit einem Eigenwert größer 1. Auch die grafische Analyse des Scree-Tests zeigt eine eindeutige Knickstelle (Ellenbogenkriterium) nach der ersten Komponente. Die einfaktorielle Lösung kann 50,8 \% der Gesamtvarianz aufklären (vgl. Pallant 2013).

In einem zweiten Schritt ist die Reliabilität zu prüfen, das heißt die Frage, wie reliabel die Skala für die gewählten Items ist. Die Grundlage bildet der 
Alpha-Koeffizient nach Cronbach. Der berechnete Wert liegt bei 0,77, was in der Fachliteratur als zufriedenstellend bewertet wird (Kopp und Lois 2014; Kuckartz et al. 2013; Pallant 2013). Dabei wurde ebenfalls geprüft, inwieweit der Ausschluss eines Items möglicherweise den Cronbach-Alpha-Wert erhöht. Bei allen Items würde der Wert sinken, was gegen den Ausschluss eines Items spricht. Der zweite wichtige Kennwert, die sogenannte Trennschärfe, gibt die Korrelation zwischen den einzelnen Items und der Gesamtskala an. Alle Werte liegen über 0,45 und damit deutlich über dem Minimalwert von 0,3, was ebenfalls gegen den Ausschluss eines Items spricht (Kopp und Lois 2014, S. 98-99).

In einem dritten Schritt erfolgt die eigentliche Skalenbildung. Dabei wird der Skalenwert als arithmetischer Mittelwert der fünf Items gebildet, und zwar aufgrund der leichteren Interpretierbarkeit im Vergleich zum Summenwert. Als Voraussetzung wird festgelegt, dass mindestens drei der fünf Items beantwortet wurden. Die Ergebnisse variieren zwischen 1 (maximale Ablehnung) und 5 (maximale Zustimmung) hinsichtlich ausgewählter NPM-Reformen. Zwecks besserer Interpretierbarkeit werden die individuellen Durchschnittswerte zwischen 1 und 5 wiederum in eine Fünferskala unterteilt (vgl. Tabelle 6.4).

Tabelle 6.4 Skala zur Beurteilung der NPM-Reformen

\begin{tabular}{l|l|l}
\hline & Spanne der Mittelwerte & Verteilung (\%) \\
\hline Sehr negativ (1) & 1 bis 1.8 & 9 \\
\hline Eher negativ (2) & $<1.8$ bis 2.6 & 16 \\
\hline Neutral (3) & $<2.6$ bis 3.4 & 41 \\
\hline Eher positiv (4) & $<3.4$ bis 4.2 & 27 \\
\hline Sehr Positiv (5) & $<4.2$ bis 5 & 6 \\
\hline
\end{tabular}

Quelle: MOBIL 2012/2013 N = 179

Die Ergebnisse zeigen, dass die mit Abstand größte Gruppe weder besonders positiv noch besonders negativ gegenüber NPM-Reformen eingestellt ist. Über ein Viertel bewertet die Reformen eher positiv, während lediglich eine kleine Gruppe von $6 \%$ vollständig von den NPM-Reformen überzeugt ist. Zugleich lehnt etwa jeder elfte Professor mit Migrationshintergrund die NPM-Reformen kategorisch ab. Professorinnen sind den NPM-Reformen gegenüber deutlich aufgeschlossener eingestellt, was sich darin äußert, dass fast die Hälfte (46\%) eine positive Beurteilung vornimmt. Große Unterschiede zeigen sich auch zwischen den Fächergruppen. Über die Hälfte der Ingenieurwissenschaftler und fast die Hälfte (44\%) der Professoren aus den Sozial-/Verhaltenswissenschaften befürwortet die NPM-Reformen. Demgegenüber ist die Mehrheit aus dem Bereich 
Kunst/Kunstwissenschaften und aus den Geisteswissenschaften gegen die genannten Reformmaßnahmen.

\subsubsection{Standpunkte zur Öffnung der Hochschule}

Die Öffnung der Hochschule stellt spätestens seit dem Beschluss der Kultusministerkonferenz von 2009 ein wichtiges Themenfeld im Rahmen der Hochschulentwicklung in Deutschland dar. Bei der Öffnung der Hochschule geht es zum einen um die Frage der Öffnung für neue bzw. bisher unterrepräsentierte Gruppen wie Studierende aus nichtakademischen Elternhäusern (Firstgeneration Studierende), Studierende mit Migrationshintergrund oder Studierende mit Beeinträchtigung. Zum anderen ist auch die Durchlässigkeit zwischen beruflicher und akademischer Bildung in diesem Kontext von zentraler Bedeutung, wozu beispielsweise die Frage zählt, inwieweit sich die Hochschule für beruflich qualifizierte Studieninteressierte ohne allgemeine Hochschulreife öffnet. Zugleich wird vor dem Hintergrund des Konzepts des „lebenslangen Lernens“ auch diskutiert, inwiefern die Hochschule sich auch für ältere Studierende öffnen bzw. auch das Angebot wissenschaftlicher Weiterbildung stärker ausbauen sollte (Schütze und Slowey 2012; Wolter et al. 2014). Dementsprechend führt die Debatte über die weitere Öffnung der Hochschule auch zu der Frage, inwieweit eine stärkere horizontale Differenzierung im Sinne einer stärkeren Profilbildung des Hochschulwesens stattfinden sollte. Ebenso lässt sich im Kontext steigender Studierendenzahlen in den letzten Jahren zunehmend eine Kontroverse unter dem Stichwort „Akademisierungswahn" beobachten. Der Kern der Debatte besteht in der Frage, inwieweit durch den steigenden Studierendenanteil eine „Entwertung“ des akademischen Abschlusses stattfindet, der in bestimmten Fächergruppen auf dem Arbeitsmarkt kaum mehr nachgefragt ist, oder ob akademischer Bildung nicht eigentlich eine zentrale Relevanz zukommt, um den zunehmend komplexeren Anforderungen auf dem Arbeitsmarkt gerecht zu werden (Wolter 2014a).

Die These, dass die Hochschulen durch die steigenden Studierendenzahlen einen Bedeutungsverlust erfahren, trifft nach Ansicht von $43 \%$ der Befragten nicht zu. Zugleich stimmt aber auch ca. ein Drittel der Professoren dieser These zu (vgl. Abbildung 6.25). Auffällig ist, dass Professoren aus Entwicklungsländern $(67 \%)$ und Professoren aus nichtakademischen Elternhäusern $(55 \%)$ der These am entschiedensten widersprechen, also zwei Gruppen, die vergleichsweise schwach unter Professoren repräsentiert sind.

Die Mehrheit der Professoren mit Migrationshintergrund plädiert für eine stärkere Öffnung der Hochschule (43\%), während knapp ein Viertel (23\%) sich 
Durch die Zunahme der Studierendenzahlen erfahren die
Hochschulen einen Bedeutungsverlust

Offnung der Hochschulen for neue Gruppen*

Etimme uberhoupt nicht zu

Stimme eher nicht zu

Entimme teilweise zu

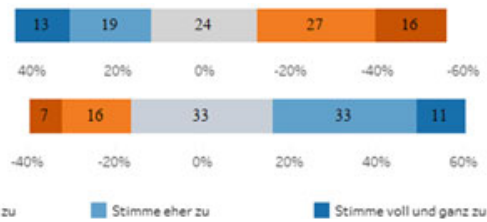

Abbildung 6.25 Einstellung zur Öffnung der Hochschule. *Legende: Sehr schlecht, eher schlecht, teils/teils, eher gut, sehr gut. (Quelle: MOBIL 2012/2013 (Item 1: N = 164 Item 2: $\mathrm{N}=163)$ )

dagegen ausspricht (vgl. Abbildung 6.25). Dabei sprechen sich Professoren an Fachhochschulen und Juniorprofessoren deutlich offensiver (jeweils über $60 \%$ ) für eine stärkere Öffnung aus als ihre Kollegen.

\subsubsection{Ansichten zur vertikalen Differenzierung der Hochschulen}

Das Hochschulwesen in Deutschland gilt traditionell und im internationalen Vergleich als vergleichsweise horizontal strukturiert. Neben der Unterteilung in Fachhochschulen und Universitäten gibt es etwa hinsichtlich der Reputation zwar informelle Unterschiede zwischen den Universitäten, dennoch fallen diese traditionell eher gering aus. Seit einigen Jahren wird zunehmend die Forderung laut, den Wettbewerb zwischen den Hochschulen und die vertikale Differenzierung zu verstärken. Die Exzellenzinitiative für deutsche Universitäten, die im Jahr 2005 von Bund und Ländern initiiert wurde, spielt diesbezüglich eine wichtige Rolle. Während hierdurch auch Prozesse der horizontalen Differenzierung angestoßen wurden, wie beispielsweise eine stärkere Profilbildung durch die Schaffung von Exzellenzclustern, lag ein Ziel der Initiative auch darin, eine stärkere vertikale Differenzierung des Universitätswesens zu etablieren, insbesondere um die internationale Wettbewerbsfähigkeit deutscher Universitäten zu stärken (Banscherus, Engel, Spexard, Wolter 2015b). Diese Zielsetzung erschließt sich vor dem Hintergrund der enormen Öffentlichkeitswirkung internationaler Forschungsrankings, in denen Deutschland zwar eine Vielzahl von überdurchschnittlich „guten“ Universitäten stellt, unter den Topplatzierungen allerdings kaum erscheint und insbesondere weit hinter Universitäten aus Großbritannien und den USA zurückbleibt. Zugleich gibt es eine große Zahl kritischer Stimmen, die die starke 
Fokussierung entsprechender Rankings auf Publikationsindexe und Drittmittel in Frage stellen.

Eine weitere zentrale Frage in diesem Kontext lautet, inwieweit private Hochschulen in Deutschland eine stärkere Rolle spielen sollten. Trotz eines enormen Anstiegs liegt der Anteil der Studierenden an privaten Fachhochschulen bei lediglich $9 \%$. Der private Hochschulsektor in Deutschland konzentriert sich primär auf die Fachrichtungen der Wirtschafts-, Ingenieur- und Gesundheitswissenschaften und ist stark auf berufsbegleitende Studienangebote ausgerichtet. Private Hochschulen betonen in ihrer Außendarstellung, neue Impulse für Innovation zu setzen und eine Bildung in den Vordergrund $\mathrm{zu}$ stellen, die den Anforderungen des Arbeitsmarktes besser gerecht werde. Kritiker verweisen hingegen auf die Gefahr einer zunehmenden sozialen Spaltung aufgrund der Studiengebühren an privaten Hochschulen (Autorengruppe Bildungsberichterstattung 2018).

Mit Abstand am positivsten wird die Exzellenzinitiative von den Professoren mit Migrationshintergrund beurteilt. Die Mehrheit (40\%) bewertet die Einführung positiv, während knapp ein Drittel (29 \%) sich hierzu eher kritisch positioniert (vgl. Abbildung 6.26). Am stärksten fällt die Zustimmung unter Professoren aus nichtakademischen Elternhäusern aus, von denen knapp die Hälfte die Initiative positiv bewertet.

Demgegenüber beurteilt eine deutliche Mehrheit Hochschulrankings negativ (44\%). Lediglich etwas über ein Viertel (28\%) spricht sich für Hochschulrankings aus (vgl. Abbildung 6.26). Deutliche Unterschiede zeigen sich zwischen den Fächergruppen. Lediglich jeder zehnte Professor aus den Geisteswissenschaften und niemand aus dem Bereich Kunst/Kunstwissenschaft steht Hochschulrankings positiv gegenüber.

Exzellenzinitiative
Hochschulrankings
Zunahme der Anzahl privater Hochschulen

Sehr schlecht

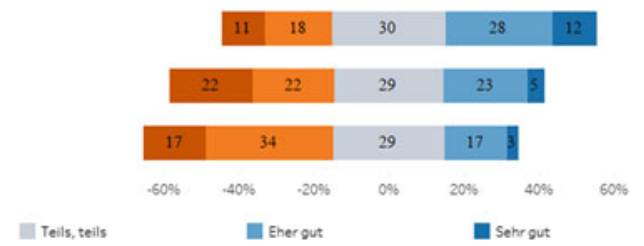

Abbildung 6.26 Beurteilung von Entwicklungen im Kontext der vertikalen Differenzierung der Hochschulen. (Quelle: MOBIL 2012/2013 (Item 1: N = 183 Item 2: N = 177 Item 3: N $=156)$ )

Am negativsten wird der Ausbau des privaten Hochschulsektors beurteilt. Über die Hälfte spricht sich dagegen und lediglich jeder fünfte Professor mit 
Migrationshintergrund dafür aus (vgl. Abbildung 6.26). Dabei muss allerdings berücksichtigt werden, dass ausschließlich Professoren an staatlichen Hochschulen befragt wurden, wodurch in der negativen Beurteilung möglicherweise auch eine gewisse Abgrenzung zum privaten Sektor zum Ausdruck kommt. Deutlich offener zeigt sich die Gruppe der Juniorprofessoren, von denen über $40 \%$ einen Ausbau privater Hochschulen positiv bewerten.

\subsection{Berufliche Zufriedenheit und Arbeitsbedingungen}

Die Frage nach der Arbeitszufriedenheit hat sich als fester Bestandteil von Befragungen in unterschiedlichen Berufsfeldern etabliert. Die Relevanz dieser Frage lässt sich insbesondere damit erklären, dass eine hohe Berufszufriedenheit nicht nur eine wichtige Bedeutung für die Attraktivität der Stelle nach außen hat, sondern auch häufig stark verbunden ist mit dem Berufserfolg und dem persönlichen Wohlbefinden. Als grundlegendes Verständnis wird dabei zumeist zugrunde gelegt, dass Zufriedenheit sich in erster Linie danach bemesse, inwieweit eine bestimmte Erwartungshaltung in der Realität erfüllt werden kann. Zudem wird häufig zwischen intrinsischen Gründen, z. B. der Möglichkeit, im Rahmen der Berufstätigkeit eigene Fähigkeiten einzubringen, und extrinsischen Gründen, z. B. der Dauer des Arbeitsvertrags oder der Höhe des Gehalts, unterschieden (Höhle und Teichler 2013a).

Um die berufliche Zufriedenheit der Professoren mit Migrationshintergrund näher zu untersuchen, wird zunächst kurz betrachtet, wie die Kernbereiche akademischer Tätigkeit, das heißt Forschung und Lehre, von ihrer Seite im internationalen Vergleich beurteilt werden (Abschnitt 6.5.1). Im zweiten Schritt soll näher beleuchtet werden, wie zufrieden Professoren mit Migrationshintergrund insgesamt mit ihrer beruflichen Situation sind. Dabei werden zunächst bivariat und im Anschluss multivariat über eine logistische Regressionsanalyse untersucht, welche migrationsspezifischen, soziodemographischen und hochschulspezifischen Gruppenunterschiede sich bei Professoren mit Migrationshintergrund zeigen (Abschnitt 6.5.2). Im Anschluss werden unterschiedliche positive und negative Items zur Beurteilung der Arbeitsbedingungen betrachtet und über Korrelationsanalysen und eine logistische Regressionsanalyse analysiert, in welcher Form die Arbeitsbedingungen die berufliche Zufriedenheit erklären können (Abschnitt 6.5.3). Abschließend werden soziodemographische Unterschiede für die fünf Items, die für die berufliche Zufriedenheit die größte Erklärungskraft aufweisen, herausgestellt (Abschnitt 6.5.4). 
Eine ausführliche Analyse der beruflichen Zufriedenheit ist auch vor dem Hintergrund relevant, dass der Grad der beruflichen Zufriedenheit Aufschluss darüber geben kann, inwieweit es für renommierte Wissenschaftler aus dem Ausland attraktiv erscheint, sich auf Professorenstellen in Deutschland zu bewerben. Die Analysen in dieser Arbeit zeigen, wie ausgeprägt die Verbindungen und Netzwerke der Professoren mit Migrationshintergrund in die internationale Scientific Community (Wissenschaftsgemeinde) sind (vgl. Abschnitt 6.6). Eine hohe berufliche Zufriedenheit der Professoren mit Migrationshintergrund erhöht somit möglicherweise auch die Chancen, dass sie weiteren Wissenschaftlern aus dem Ausland eine berufliche Tätigkeit in Deutschland nahelegen.

\subsubsection{Beurteilung der Forschungs- und Lehrbedingungen im internationalen Vergleich}

Die Frage nach der Beurteilung der Forschungs- und Lehrbedingungen im internationalen Vergleich ist insbesondere aufgrund der Tatsache von Interesse, dass eine Vielzahl der Professoren mit Migrationshintergrund sowohl im Studium als auch beruflich Erfahrungen und Einblicke in Hochschulwesen aus anderen Ländern zugrunde legen kann.

Die Forschungs- sowie die Lehrbedingungen im internationalen Vergleich werden jeweils von ca. $40 \%$ der Professoren kritisch beurteilt, während knapp $30 \%$ der Befragten die Bedingungen positiv bewerten (vgl. Abbildung 6.27).

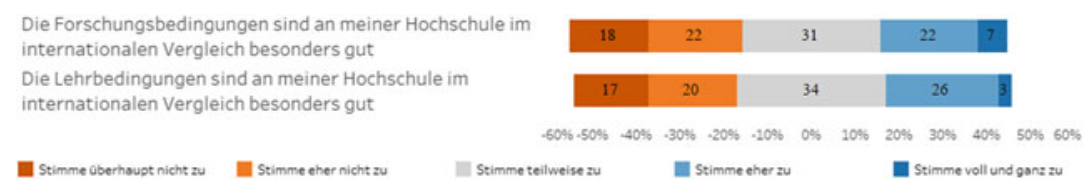

Abbildung 6.27 Beurteilung der Forschungs- und Lehrbedingungen. (Quelle: MOBIL 2012/2013 (Item 1: N = 162 Item 2: $N=164$ ))

Dabei zeigen sich deutliche Unterschiede zwischen den Fächergruppen. Die Forschungsbedingungen werden mit großem Abstand am häufigsten von Professoren aus den Sozial- und Verhaltenswissenschaften (66 \%) kritisiert. Demgegenüber beschreiben über $40 \%$ der Professoren aus dem Bereich Kunst/Kunstwissenschaften, den Naturwissenschaften und den Ingenieurwissenschaften die Forschungsbedingungen im internationalen Vergleich als besonders 
gut. Kritische Positionen zu den Forschungsbedingungen finden sich zudem besonders häufig bei Professorinnen (45\%) und Fachhochschulprofessoren $(72 \%)$. Gute Lehrbedingungen werden ebenfalls in besonderem Maße von Professoren aus dem Bereich Kunst/Kunstwissenschaft (70 \%) hervorgehoben. Demgegenüber liegt der Anteil positiver Beurteilungen der Lehrbedingungen in den Lebenswissenschaften, den Geisteswissenschaften und den Sozial/Verhaltenswissenschaften jeweils bei unter $20 \%$. Zwischen Herkunftsländern zeigen sich hingegen in beiden Feldern kaum Unterschiede. Auffällig sind allerdings die Unterschiede zwischen den Migrationstypen im Feld der Lehre. Die Lehrbedingungen werden von Early Migrants deutlich negativer beurteilt als von Student und Professional Migrants. Es ist interessant, dass über ein Viertel der Early Migrants, also aus der Gruppe mit den in Relation geringsten Erfahrungen in Hochschulsystemen außerhalb Deutschlands (vgl. Abschnitt 6.2 und 6.6.1), die Lehrbedingungen an der Hochschule im internationalen Vergleich in hohem Maße kritisiert, während die Anteile bei den später zugewanderten Professoren bei unter $15 \%$ liegen.

\subsubsection{Zusammenhang zwischen soziodemographischen Merkmalen und beruflicher Zufriedenheit}

Im nächsten Schritt steht die generelle berufliche Zufriedenheit im Mittelpunkt der Analyse, die fast durchgehend in Hochschullehrerbefragungen erhoben wird. Die berufliche Zufriedenheit stellt nicht nur einen Indikator für die Attraktivität der Profession dar, sondern steht auch in enger Verbindung zum beruflichen Erfolg und persönlichem Wohlbefinden (vgl. Höhle und Teichler 2013a).

Insgesamt sind ca. drei Viertel $(74 \%)$ der Professoren mit ihrer beruflichen Situation eher $(51 \%)$ oder voll und ganz zufrieden $(23 \%)$. Von den übrigen $26 \%$ sind lediglich $2 \%$ überhaupt nicht zufrieden und $12 \%$ eher nicht zufrieden. Das arithmetische Mittel auf der Skala zwischen 1 (sehr zufrieden) und 5 (überhaupt nicht zufrieden) liegt bei 2,19. Die Zufriedenheit fällt damit geringfügig höher aus als bei den in der CAP-Studie befragten Senior Academics in Deutschland $(2,24)$ (Höhle und Teichler 2013a). Im Folgenden werden lediglich zwei Gruppen unterschieden, nämlich erstens Professoren, die mit ihrer beruflichen Situation zufrieden sind, und zweitens Professoren, die sich nicht oder nur "teils/teils“ zufrieden zeigen. Diese Zusammenfassung wurde aus statistischen Gründen vorgenommen, da für die folgende multivariate logistische Regressionsanalyse die Größe der fünf Teilgruppen nicht ausreichend war. 
Kaum Unterschiede hinsichtlich der Zufriedenheit zeigen sich beim Zeitpunkt der Zuwanderung, der Nationalität oder dem Herkunftsland. Dies gilt ebenfalls für den Vergleich der drei Altersgruppen. Professoren aus akademischen Elternhäusern sind hingegen deutlich seltener mit ihrer beruflichen Situation zufrieden als Professoren der First Generation. Zwischen Universitätsprofessoren und Fachhochschulprofessoren lassen wie auch bei der CAP-Studie (Höhle und Teichler 2013a). keine Unterschiede bei der beruflichen Zufriedenheit feststellen. Die größten Unterschiede werden zwischen den Besoldungsgruppen sichtbar. W3/C4-Professoren sind mit Abstand am häufigsten zufrieden. Die Zufriedenheit von W2-/C3-/C2-Professoren liegt an Universitäten etwas unter dem Durchschnitt und entspricht an Fachhochschulen in etwa dem Durchschnitt. Mit Abstand am unzufriedensten sind Juniorprofessoren, von denen sich weniger als die Hälfte mit ihrer beruflichen Situation zufrieden zeigt. Hier liegt die Vermutung nahe, dass

Tabelle 6.5 Berufliche Zufriedenheit nach soziodemographischen Merkmalen

\begin{tabular}{|c|c|c|c|}
\hline & Anteil beruflich zufrieden ${ }^{a}$ & $\mathrm{~N}$ & Sig. \\
\hline Gesamt & $74 \%$ & 185 & \\
\hline Early Migrants $<18$ & $70 \%$ & \multirow[t]{3}{*}{185} & \multirow[t]{3}{*}{0.513} \\
\hline Student Migrants 18-31 & $72 \%$ & & \\
\hline Professional Migrants 32+ & $79 \%$ & & \\
\hline Max. 10 Jahre in Deutschland & $74 \%$ & \multirow[t]{4}{*}{183} & \multirow[t]{4}{*}{0.820} \\
\hline Zwischen 11-20 Jahren & $79 \%$ & & \\
\hline Über 20 Jahre in Deutschland & $72 \%$ & & \\
\hline Zweite Generation & $71 \%$ & & \\
\hline Deutsch mit MgH & $78 \%$ & \multirow[t]{3}{*}{185} & \multirow[t]{3}{*}{0.489} \\
\hline Ausländisch & $74 \%$ & & \\
\hline Deutsche und ausländische StA. & $67 \%$ & & \\
\hline Österreich und Schweiz & $67 \%$ & \multirow[t]{5}{*}{185} & \multirow[t]{5}{*}{0.253} \\
\hline Weitere Länder Westeuropas & $71 \%$ & & \\
\hline Osteuropa & $89 \%$ & & \\
\hline Lateinamerika, Asien und Afrika & $68 \%$ & & \\
\hline $\begin{array}{l}\text { Angelsächsiche Länder (USA, } \\
\text { Kanada und Australien) }\end{array}$ & $79 \%$ & & \\
\hline Industrieland & $73 \%$ & 185 & 0.963 \\
\hline
\end{tabular}


Tabelle 6.5 (Fortsetzung)

\begin{tabular}{|c|c|c|c|}
\hline & Anteil beruflich zufrieden ${ }^{a}$ & $\mathrm{~N}$ & Sig. \\
\hline Schwellen-/Entwicklungsland & $74 \%$ & & \\
\hline Nicht muslimisches Herkunftsland & $73 \%$ & \multirow[t]{2}{*}{185} & \multirow[t]{2}{*}{0.922} \\
\hline Muslimisches Herkunftsland & $75 \%$ & & \\
\hline Jüngere Generation $<=44$ & $75 \%$ & \multirow[t]{3}{*}{184} & \multirow[t]{3}{*}{0.875} \\
\hline Mittlere Generation 45-53 & $75 \%$ & & \\
\hline Ältere Generation 54+ & $71 \%$ & & \\
\hline Männer & $78 \%$ & \multirow[t]{2}{*}{184} & \multirow[t]{2}{*}{$0.044 *$} \\
\hline Frauen & $64 \%$ & & \\
\hline Kein Hochulabschluss & $84 \%$ & \multirow[t]{2}{*}{163} & \multirow[t]{2}{*}{$0.089 *$} \\
\hline $\begin{array}{l}\text { Mindestens ein Elternteil } \\
\text { Hochschulabschluss }\end{array}$ & $72 \%$ & & \\
\hline Juniorprofessur (Uni) & $46 \%$ & \multirow[t]{4}{*}{181} & \multirow[t]{4}{*}{$0.001 * * *$} \\
\hline Fachhochschulprofessur & $73 \%$ & & \\
\hline W2/C3/C2-Professur (Uni) & $67 \%$ & & \\
\hline W3/C4-Professur (Uni) & $87 \%$ & & \\
\hline Geisteswissenschaften & $71 \%$ & \multirow[t]{6}{*}{178} & \multirow[t]{6}{*}{0.665} \\
\hline Sozial-/Verhaltenswissenschaften & $68 \%$ & & \\
\hline Kunst/Kunstwissenschaften & $77 \%$ & & \\
\hline Lebenswissenschaften & $78 \%$ & & \\
\hline Naturwissenschaften & $72 \%$ & & \\
\hline Ingenieurwissenschaften & $88 \%$ & & \\
\hline
\end{tabular}

Quelle: MOBIL 2012/2013; Signifikanzlevel: *p $\leq 0,1, * * p \leq 0,01, * * * p \leq 0,001$. $^{\text {a }}$ (Personen, die mit ihrer beruflichen Situation eher zufrieden (4) oder voll und ganz zufrieden (5) sind)

die geringere Vergütung sowie die häufig befristeten Stellen z. T. ohne TenureTrack-Option wichtige Faktoren für die größere Unzufriedenheit darstellen (vgl. Tabelle 6.5).

Im Folgenden soll über eine logistische Regressionsanalyse näher untersucht werden, inwieweit die Unterschiede sich nicht nur in der bivariaten Analyse zeigen, sondern auch im Rahmen einer multivariaten Regressionsanalyse sichtbar werden. Dabei werden average marginal effects (AME) verwendet (vgl. ausführlich Abschnitt 5.3). AME werden in durchschnittlichen Prozentpunkten dargestellt und geben an, in welchem Maße sich die Wahrscheinlichkeit, beruflich zufrieden 
zu sein, zwischen den Gruppen unterscheidet. Über das multivariate Verfahren kann unter anderem untersucht werden, inwieweit bspw. die geringere Zufriedenheit von Frauen auf geschlechtsspezifische Gründe zurückzuführen ist oder ob dies möglicherweise damit zusammenhängt, dass der Frauenanteil in der „unzufriedensten“ Besoldungsgruppe W1 mit Abstand am höchsten ausfällt.

In Anlehnung an die Modellbildung der Regressionsanalyse nach Heeringa, West und Berglund (2010, S. 210) werden ausschließlich unabhängige Variablen berücksichtigt, deren bivariater Zusammenhang ein Signifikanzniveau von $\mathrm{p}<0,25$ erreicht. Auf der Grundlage der bivariaten Analyse (vgl. Tabelle 6.5) wird bei der Regressionsanalyse dementsprechend ausschließlich der Einfluss des Geschlechts, der sozialen Herkunft und der Besoldungsgruppe untersucht. Auf dieser Grundlage werden drei Modelle verglichen. Modell 1 untersucht den Einfluss des Geschlechts auf die berufliche Zufriedenheit, Modell 2 den multivariaten Einfluss von Geschlecht und sozialer Herkunft und Modell 3 den multivariaten Einfluss aller drei Variablen. Die Entwicklung der Modellgüte wird über Pseudo$\mathrm{R}^{2}$ bestimmt, wobei ein Anstieg des Wertes auf eine Verbesserung der Modellgüte hinweist (vgl. ausführlich Long und Freese 2014, S. 122-124).

Modell 1 zeigt einen signifikanten Zusammenhang zwischen Geschlecht und beruflicher Zufriedenheit. Die Wahrscheinlichkeit, dass Professorinnen mit Migrationshintergrund zufrieden sind, fällt durchschnittlich um 14 Prozentpunkte geringer aus als bei ihren männlichen Kollegen. Die Wahrscheinlichkeit bleibt weiterhin signifikant und steigt sogar noch auf 16 Prozentpunkte an, wenn der multivariate Einfluss von Geschlecht und sozialer Herkunft (Modell 2) berücksichtigt wird. Wenn allerdings neben der sozialen Herkunft auch die Besoldungsgruppen berücksichtigt werden (Modell 3), liegt die Wahrscheinlichkeit nur noch bei 9 Prozentpunkten und unterscheidet sich nicht mehr signifikant (vgl. Tabelle 6.6). Der größte Unterschied zeigt sich, wie bei der bivariaten Analyse, zwischen Juniorprofessoren und W3-/C4-Professoren. Die entsprechende Wahrscheinlichkeit, beruflich zufrieden zu sein, liegt bei W3-/C4-Professoren um 27 Prozentpunkte höher als bei Juniorprofessoren. Hinsichtlich der Geschlechterunterschiede bleibt festzuhalten, dass Professorinnen mit Migrationshintergrund häufiger unzufrieden sind als ihre männlichen Kollegen, die Unterschiede aber zu einem großen Teil damit zusammenhängen, dass Frauen deutlich häufiger eine W1-Professur und zugleich deutlich seltener eine W3-/C4-Professur innehaben (vgl. Tabelle 6.6).

Hinsichtlich der W1-Professuren ist zu berücksichtigten, dass diese als alternative Qualifikationsphase zur Habilitation angelegt sind. Dementsprechend legt der hohe Frauenanteil in diesem Bereich durchaus den Schluss nahe, dass sich 
Tabelle 6.6 Binäre logistische Regression über den Einfluss von Geschlecht, sozialer Herkunft und Besoldungsgruppe auf die berufliche Zufriedenheit (average marginal effects)

\begin{tabular}{l|l|l|l}
\hline & Model 1 & Model 2 & Model 3 \\
\hline Professorinnen (Ref.: Professoren) & $-0.14^{*}$ & $-0.16^{*}$ & -0.09 \\
\hline Akademisches Elternhaus (Ref.: First Generation) & & $-0.14^{*}$ & $-0.13^{*}$ \\
\hline Besoldungsgruppe (Ref.: Juniorprofessur) & & & \\
\hline Fachhochschulprofessur & & & 0.13 \\
\hline W2/C3/C2-Professur (Uni) & & & 0.09 \\
\hline W3/C4-Professur & & & $0.27^{*}$ \\
\hline Pseudo R`2 & 0.021 & 0.045 & 0.068 \\
\hline $\mathrm{N}$ & 159 & 159 & 159 \\
\hline
\end{tabular}

Quelle: MOBIL 2012/2013; Signifikanzlevel: *p $\leq 0,1, * * p \leq 0,01 . * * * p \leq 00,001$

der Anteil von Professorinnen mit Migrationshintergrund auf der Ebene der Vollprofessuren weiter erhöhen wird. Kritisch ist indessen der sehr geringe Anteil an W3-/C4-Professorinnen zu sehen. Auch bei allen Professoren bundesweit zeigt sich, dass der Frauenanteil mit steigender Besoldungsgruppe zurückgeht, so liegt der Frauenanteil für das Jahr 2017 unter W1-Professoren bei 44,5 \%, bei W2-Professoren bei 27,2 \% und bei W3-Professoren bei 22,8 \% (Statistisches Bundesamt 2018b). Nur über einen Strukturwandel in den Besoldungsgruppen wird es möglich sein, dass die Geschlechtszugehörigkeit auch hinsichtlich der beruflichen Zufriedenheit weitgehend irrelevant wird. Zusammenfassend bleibt festzuhalten, dass bei der beruflichen Zufriedenheit von Professoren mit Migrationshintergrund grundlegende Unterschiede nach Geschlecht, Bildungsherkunft und Besoldungsgruppe sichtbar werden. Dabei spielt die Besoldungsgruppe mit Abstand die wichtigste Rolle bei der Zufriedenheit. Dennoch lässt sich anhand der kleinen Pseudo- $\mathrm{R}^{2}$ festhalten, dass der Erklärungsbeitrag der demographischen Merkmale begrenzt ist. Aufgrund dessen soll im Folgenden der Zusammenhang zwischen den Arbeitsbedingungen und der beruflichen Zufriedenheit näher betrachtet werden, um wichtige Einflussfaktoren für die berufliche Zufriedenheit näher beschreiben zu können. 


\subsubsection{Zusammenhang zwischen Arbeitsbedingungen und beruflicher Zufriedenheit}

In einem nächsten Schritt werden die Arbeitsbedingungen von Professoren mit Migrationshintergrund näher betrachtet, um auf dieser Grundlage zu analysieren, in welcher Form die Arbeitsbedingungen die berufliche Zufriedenheit beeinflussen. Es wurden insgesamt 26 Items erhoben, die spezifische Aspekte der Arbeitsbedingungen von Professoren zum Gegenstand haben. Die Konstruktion der Items basiert auf einer Studie von Schaeper (1995) zur Arbeitssituation und Faktoren der beruflichen Zufriedenheit von Lehrenden an westdeutschen Universitäten. Die theoretische Konstruktion basiert auf dem Konzept von Weichert (2017), die in ihrem Beitrag „Arbeitsalltag und berufliche Zufriedenheit von Universitätsprofessor/innen mit Migrationshintergrund“ Unterschiede hinsichtlich der Arbeitssituation je nach Hochschulart und Besoldungsgruppe sichtbar macht. Zudem zeigt sie in Anlehnung an die Studie von Schaeper (1995) aus den 1990er-Jahren auf, wie sich die Arbeitssituation in den letzten Jahren tendenziell verändert hat. Abschließend ermittelt sie über eine Faktorenanalyse auf der Grundlage der 26 Items drei zentrale Faktoren hinsichtlich der Arbeitssituation: erstens „Strukturelle Rahmenbedingungen“, zweitens „Kommunikation und Kooperation“ und drittens „Spielräume und Perspektiven“.

Im Rahmen dieser vorliegenden Arbeit sollen darüber hinaus unmittelbare Zusammenhänge zwischen unterschiedlichen Aspekten der Arbeitsbedingungen und der beruflichen Zufriedenheit analysiert werden. In einem ersten Schritt werden die 26 Items in positive und negative Aspekte ${ }^{16}$ der Arbeitsbedingungen unterteilt. In einem zweiten Schritt wird dann die Korrelation zwischen den auf einer Likert-Skala erhobenen Items und der beruflichen Zufriedenheit ${ }^{17}$ berechnet.

Bei den positiven Aspekten der Arbeitsbedingungen wird von den Professoren mit Migrationshintergrund insbesondere die Freiheit in der Forschung, die Selbständigkeit und die Tätigkeitsvielfalt hervorgehoben. Deutlich kritischer beurteilen die Befragten die Rückmeldung über Leistungen in der Forschung, die Möglichkeit, sich beruflich weiterzuentwickeln, und die Bezahlung. Auf Routine im Berufsalltag verweist nur eine geringe Zahl der Professoren. Das Item „Routine“ wurde dabei vom Autor als positiver Aspekt der Arbeitsbedingungen eingeordnet. Interessant ist dabei, dass die Routine das einzige „positive“ Item darstellt, bei dem eine negative Korrelation zur beruflichen Zufriedenheit

\footnotetext{
${ }^{16}$ Jeweils 13 Items wurden auf beide Ebenen verteilt.

${ }^{17}$ Zufriedenheit wird hier binär codiert $(0=$ unentschieden oder eher nicht zufrieden, $1=$ zufrieden, eher zufrieden).
} 
sichtbar wird. Je stärker die Teilnehmer eine Routine in ihrem Arbeitsalltag wahrnehmen, desto seltener sind sie mit ihrer beruflichen Situation zufrieden. Die Beobachtung deutet darauf hin, dass Routine stärker im Sinne einer einseitigen, sich ständig wiederholenden Tätigkeit verstanden wird und weniger als eine Vertrautheit mit den Arbeitsbedingungen im positiven Sinne, was dafür spricht, das Item in zukünftigen Studien eher als ,zufriedenheitsbeeinträchtigende“ Variable $\mathrm{zu}$ verorten.

Die stärkste Korrelation hinsichtlich der beruflichen Zufriedenheit zeigt sich für die Items „Möglichkeit, beruflich weiterzukommen“, ,genügend Rückmeldung über Leistungen in der Forschung“, ,angemessene Bezahlung“ und ,gute Kooperation im Kollegium“ (vgl. Tabelle 6.7).

Bei den negativen Aspekten werden am häufigsten ,Zeitdruck“, ,,zu wenig Zeit für Forschung“ und „Arbeitsüberlastung“ hervorgehoben. Demgegenüber werden Konkurrenz unter Kolleginnen und Kollegen oder auch Isolation deutlich seltener als Probleme genannt. Der Zusammenhang in Form negativer Korrelation mit der beruflichen Zufriedenheit zeigt sich nach der Korrelation nach Spearmann-Rho am stärksten für die Items, ,geringe institutionelle Anerkennung für Leistungen in der Lehre“, „mangelndes Interesse der Kolleginnen und Kollegen am wissenschaftlichen Austausch“, „Isolation“, „Konflikte zwischen Forschungs- und Lehraufgaben“" und „,zu wenig Zeit für Forschung“ (vgl. Tabelle 6.8).

Die Korrelation der hervorgehobenen positiven und negativen Items der Arbeitsbedingungen mit der beruflichen Zufriedenheit deutet auf einen Zusammenhang hin. Um den Zusammenhang noch näher zu untersuchen, wird im Folgenden geprüft, inwieweit auch multivariat ein Zusammenhang zwischen den genannten Items und der beruflichen Zufriedenheit besteht oder ob - wie beispielsweise bei den Geschlechterunterschieden und den Besoldungsgruppen möglicherweise der Einfluss eines Items auf die berufliche Zufriedenheit primär über einen anderen Faktor zu erklären ist. Über die Berechnung soll zudem durch die Berechnung der Modellgüte eine Orientierung gegeben werden, inwieweit bestimmte Aspekte der Arbeitsbedingungen die berufliche Zufriedenheit erklären können.

Um dieser Frage nachzugehen, werden eine logistische Regressionsanalyse durchgeführt und wie bei der Analyse der soziodemographischen Faktoren wiederum average marginal effects (AME) berechnet. Als unabhängige Variablen 


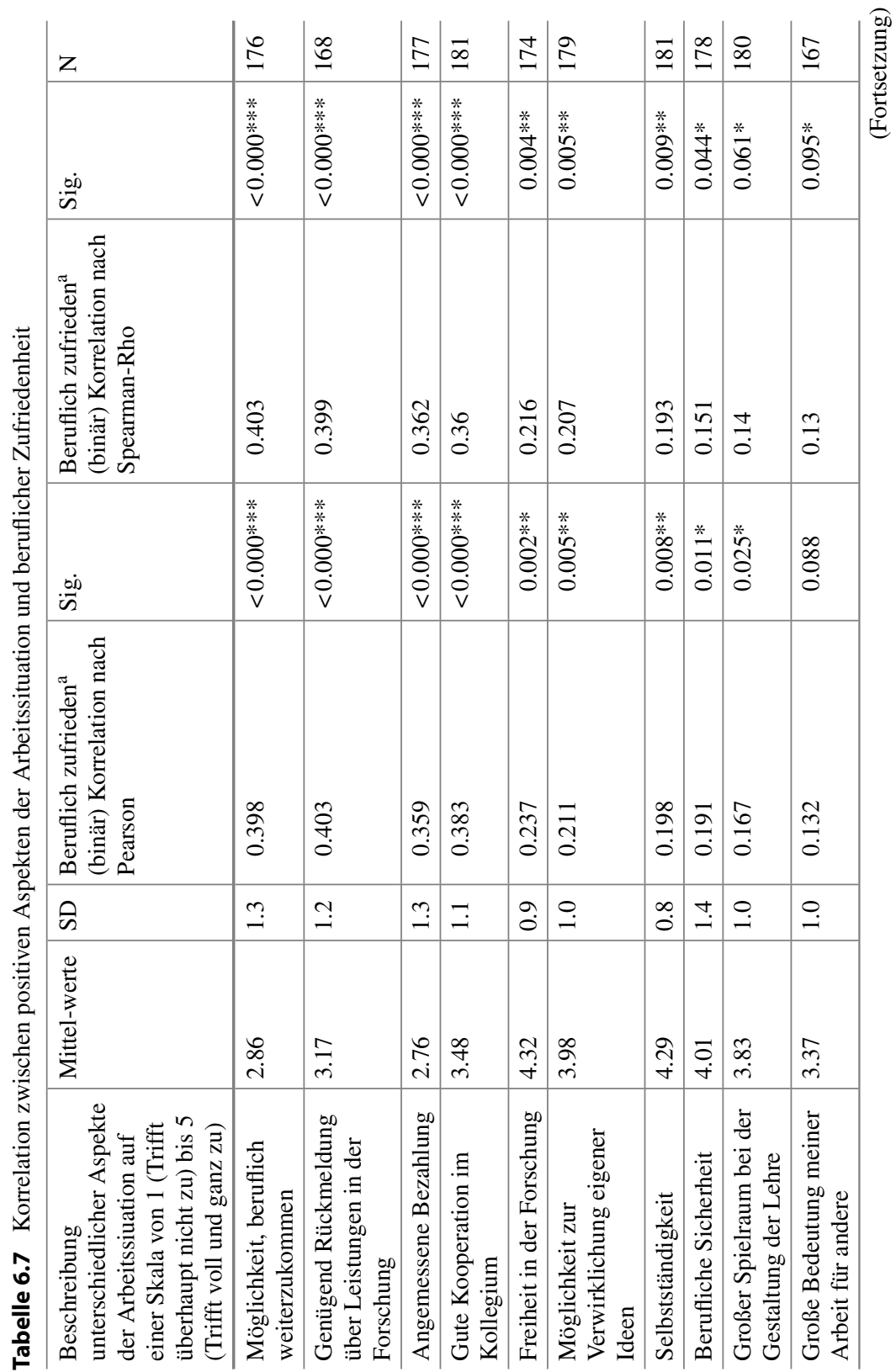




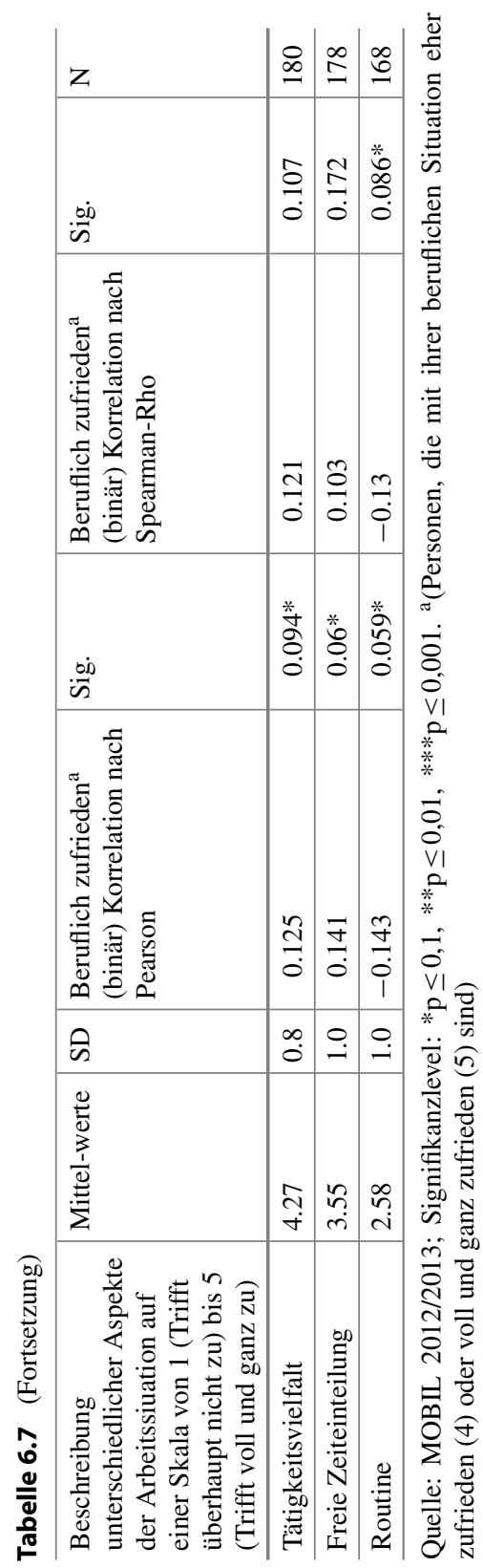




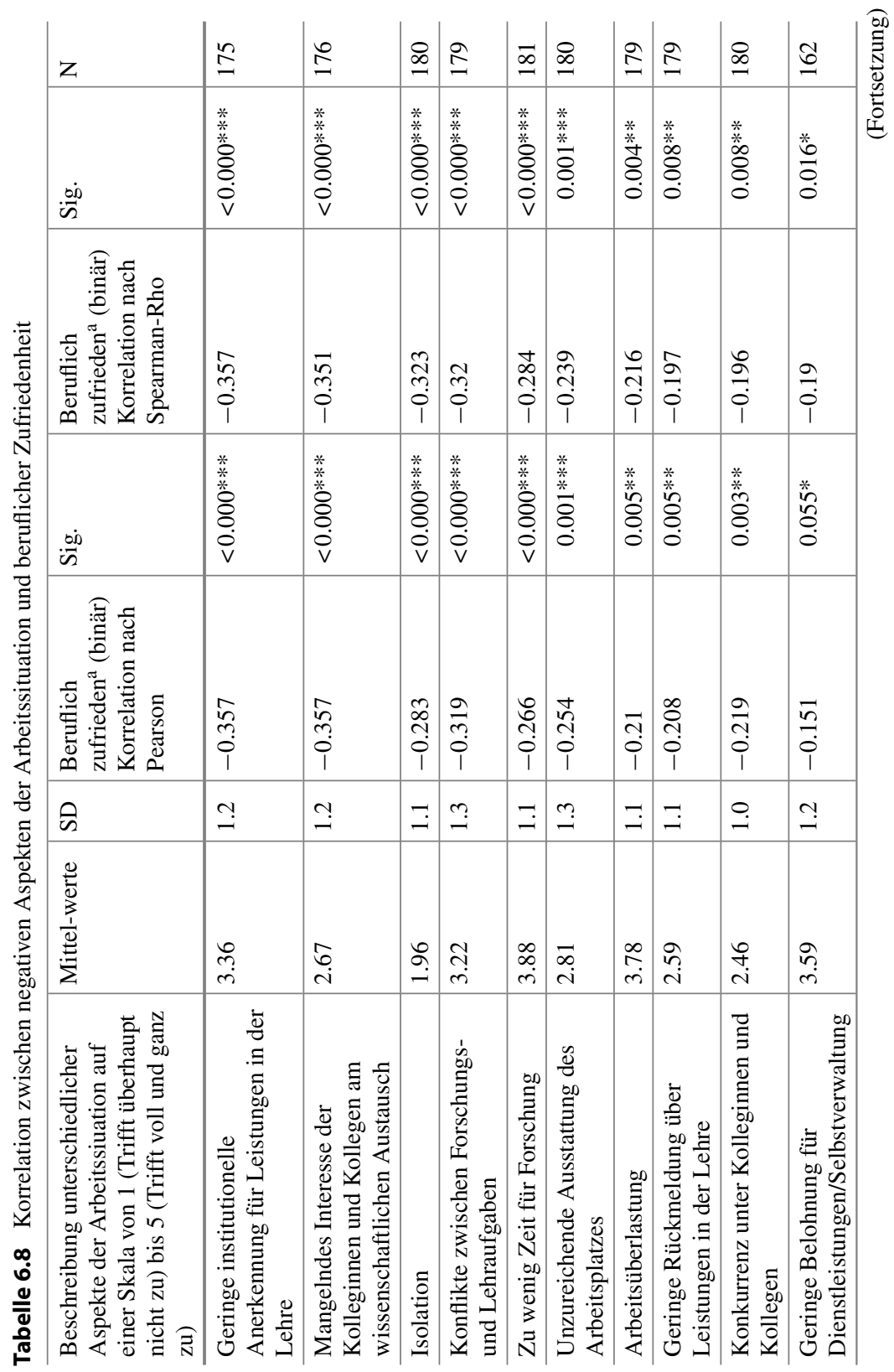




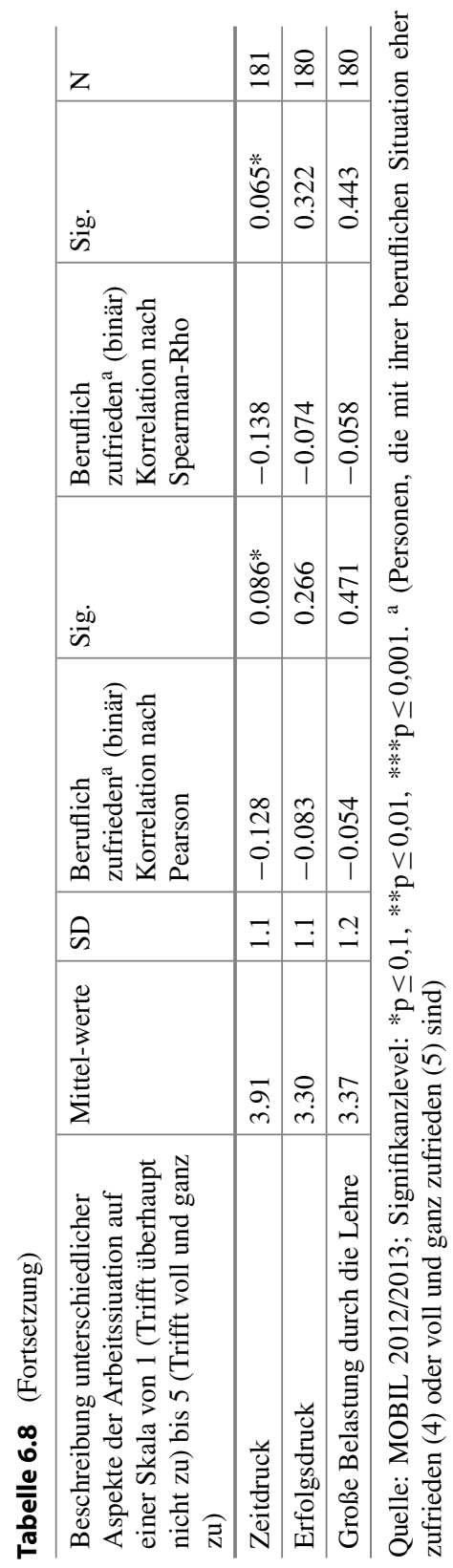


wurden die insgesamt neun Items mit den größten Korrelationskoeffizienten ausgewählt ${ }^{18}$, bei denen sich durchgehend ein hochsignifikanter Zusammenhang mit der beruflichen Zufriedenheit zeigt.

Im Anschluss wird nach dem Konzept von Long und Freese (2014) eine schrittweise Modellbildung vorgenommen. Die neun ausgewählten Items werden zunächst nach der Höhe der Korrelationskoeffizienten ${ }^{19}$ geordnet. Ausgehend vom Item mit dem größten Korrelationskoeffizienten (,Möglichkeit, beruflich weiterzukommen") wird bei jedem darauffolgenden Modell das Item mit dem nächst kleineren Koeffizienten hinzugefügt. Die Entwicklung der Modellgüte wird dabei über das „Akaike's information criterion“ (AIC) und das „Bayesian information criterion" (BIC) sowie Pseudo- $\mathrm{R}^{2}$ bestimmt. Während in Bezug auf AIC und BIC ein kleinerer Wert auf eine bessere Modellgüte hinweist, bedeutet für Pseudo$\mathrm{R}^{2}$ ein Anstieg des Wertes eine Verbesserung der Modellgüte (vgl. ausführlich Long und Freese 2014, S. 122-124). Zudem wurde für die ausgewählten Items über Streudiagramm-Analysen geprüft, inwieweit die Wahrscheinlichkeit beruflicher Zufriedenheit für die vier positiven Items konstant zu- und für die fünf negativen Items konstant abnimmt ${ }^{20}$ (Kohler und Kreuter 2017).

Die höchste Modellgüte wird bei fünf unabhängigen Variablen erreicht. $\mathrm{Ab}$ hier verschlechtern sich die genannten Indikatoren der Modellgüte. Bei den fünf ausgewählten Items (vgl. Tabelle 6.9) liegen die average marginal effects konstant zwischen 5 und 15 Prozentpunkten. Das abschließende Modell 5 zeigt, dass alle fünf Items auch multivariat einen signifikanten Einfluss auf die berufliche Zufriedenheit haben. Sobald sich die Zustimmung zu einem der fünf Items auf der Fünfer-Skala um nur eine Einheit erhöht, steigt die Wahrscheinlichkeit der beruflichen Zufriedenheit um 5 bis 7 Prozentpunkte.

Insgesamt lässt sich auf der Grundlage des Modells festhalten, dass die fünf genannten Items für die berufliche Zufriedenheit von großer Bedeutung sind, worauf der hohe Pseudo- $\mathrm{R}^{2}$-Wert von 0,33 deutlich verweist.

Kritisch lässt sich hinsichtlich des Regressionsmodells einwenden, dass die zuvor ermittelten soziodemographischen Unterschiede nicht im selben Modell berücksichtigt wurden. Der Grund dafür liegt darin, dass aufgrund der geringen Fallzahl unter Berücksichtigung der soziodemographischen Merkmale die

\footnotetext{
${ }^{18}$ Dabei handelt es sich um vier positive und fünf negative Items, die zuvor im Text hervorgehoben wurden.

${ }^{19}$ Grundlage bildet der Korrelationskoeffizient nach Spearman-Rho.

${ }^{20}$ Lediglich bei dem Item Isolation zeigt sich ein leicht U-förmiger Verlauf. Da dieses Item aber den geringsten Korrelationskoeffizienten aufweist, war dieser Befund für die weitere Modellbildung nicht relevant.
} 
Tabelle 6.9 Binäre logistische Regression über den Einfluss der Arbeitsbedingungen auf die berufliche Zufriedenheit (average marginal effects)

\begin{tabular}{l|l|l|l|l|l}
\hline & Model 1 & Model 2 & Model 3 & Model 4 & Model 5 \\
\hline $\begin{array}{l}\text { Möglichkeit, beruflich } \\
\text { weiterzukommen }\end{array}$ & $0.14 * *$ & $0.10^{* * *}$ & $00.8^{* * *}$ & $0.07 * *$ & $0.07 * *$ \\
\hline $\begin{array}{l}\text { Genügend Rückmeldung über } \\
\text { Leistungen in der Forschung }\end{array}$ & & $0.10^{* * *}$ & $0.08^{* *}$ & $0.07 * *$ & $0.06^{*}$ \\
\hline Angemessene Bezahlung & & & $0.07 * *$ & $0.05^{*}$ & $0.05^{*}$ \\
\hline Gute Kooperation im Kollegium & & & & $0.08^{* *}$ & $0.07 *$ \\
\hline $\begin{array}{l}\text { Geringe institutionelle Anerkennung } \\
\text { für Leistungen in der Lehre }\end{array}$ & & & & & $-0.06^{*}$ \\
\hline BIC & 169.1 & 161.1 & 158.8 & 156 & 156.8 \\
\hline AIC & 163 & 152 & 146.6 & 140.7 & 138.5 \\
\hline Pseudo R`2 & 0.16 & 0.22 & 0.26 & 0.3 & 0.33 \\
\hline $\mathrm{N}$ & 157 & 157 & 157 & 157 & 157 \\
\hline
\end{tabular}

Quelle: MOBIL 2012/2013. Signifikanzlevel: $* \mathrm{p} \leq 0,1, * * \mathrm{p} \leq 0,01, * * * \mathrm{p} \leq 0,001$

Modellgüte deutlich zurückgehen würde, so dass keine zuverlässigen Aussagen mehr getroffen werden könnten. Um dennoch den Zusammenhang zwischen soziodemographischen Merkmalen und den zentralen Aspekten der Arbeitsbedingungen herauszuarbeiten, werden im Folgenden zentrale soziodemographische Unterschiede der fünf Items dargestellt.

\subsubsection{Soziodemographische Unterschiede bei der Beurteilung der Arbeitsbedingungen}

Generell zeigen sich die geringsten Zustimmungswerte für die Items ,Möglichkeit, beruflich weitzukommen“, ,,angemessene Bezahlung“ und ,genügend Rückmeldung über Leistungen in der Forschung“" (vgl. Tabelle 6.7). Das heißt, wenn von hochschulpolitischer Seite das Ziel besteht die berufliche Zufriedenheit der Professoren mit Migrationshintergrund grundlegend $\mathrm{zu}$ verbessern, könnten Maßnahmen in diesen drei Feldern besonderes Potential bieten. Dabei stellt sich die Frage, inwieweit es spezifische Gruppen gibt, die die fünf herausgestellten Aspekte der Arbeitsbedingungen, in besonderem Maße positiv oder negativ beurteilen, um auf dieser Grundlage noch spezifischere Hinweise für mögliche Hochschulsteuerung zu erhalten. Um dieser Frage nachzugehen, wird 
abschließend untersucht, in welchem Maße sich bei der Einschätzung der Items soziodemographische Unterschiede zeigen. Ziel der Differenzierung ist es, herauszuarbeiten, welche Gruppen einzelne Items besonders kritisch beurteilen, um auf dieser Grundlage sichtbar zu machen, ob möglicherweise Bedarf im Bereich hochschulpolitischer Maßnahmen besteht.

Die Möglichkeit, beruflich weiterzukommen, besteht nach Ansicht der jüngeren Generation in deutlich höherem Maße als nach Ansicht der älteren Generation. Während knapp 40 \% der Juniorprofessoren und der W3-/C4-Professoren dieser These nicht zustimmen können, zeigt sich mit Abstand die größte Unzufriedenheit bei Fachhochschulprofessoren, von denen fast zwei Drittel kaum berufliche Weiterentwicklungsmöglichkeiten sehen. An dieser Stelle lässt sich nicht im Detail aufklären, worin die Gründe für die kritische Perspektive der Fachhochschulprofessoren liegen und welche beruflichen Perspektiven sie konkret vermissen. Dennoch sollte diese Erkenntnis als wichtiger Hinweis für zukünftige Erhebungen verstanden werden, diesen Aspekt detaillierter zu erforschen (vgl. Abbildung 6.28).

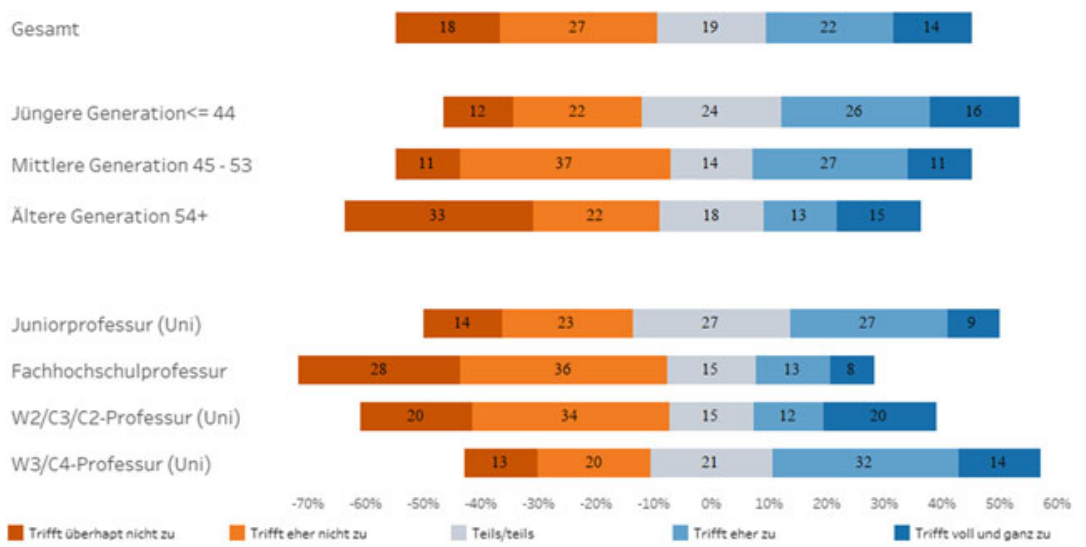

Abbildung 6.28 Soziodemographische Unterschiede hinsichtlich der Beurteilung des Items „Möglichkeit, beruflich weiterzukommen“. (Quelle: MOBIL 2012/2013 (Gesamt: N = 176, Alter: $\mathrm{N}=176(\mathrm{p}=0,062)$, Besoldungsgruppe: $\mathrm{N}=173(\mathrm{p}=0,019)$, $\mathrm{p}$-value basiert auf Kruskal-Wallis-Test)

Hinsichtlich des Feedbacks in der Forschung beurteilen die Professoren mit Migrationshintergrund ihre Situation insgesamt etwas positiver. Besonders unzufrieden zeigen sich allerdings Professoren aus den USA, Kanada und Australien. 
Diesbezüglich wäre interessant herauszufinden, inwieweit die Unzufriedenheit aus einem direkten Vergleich mit stärker ausgeprägten Feedback- und ReviewKulturen in angelsächsischen Ländern resultiert und inwiefern entsprechende Kulturen möglicherweise auch für das Wissenschaftssystem in Deutschland Potentiale bieten. Ferner zeigt sich, dass speziell Juniorprofessoren besonders unzufrieden mit den Rückmeldungen über eigene Forschungsleistungen sind. Hier stellt sich die Frage, ob sich ähnliche Befunde auch für Juniorprofessoren ohne Migrationshintergrund zeigen, um herauszufinden, ob es sich dabei nicht möglicherweise um ein primär strukturell bedingtes Problem des Stellenformates handelt (vgl. Abbildung 6.29).

Gesamt

Angelsachsiche Lander (USA, Kanada,Australien)

Lateinamerika, Asien und Afrika

Osterreich und Schweiz

Osteuropa

Weitere Lander Westeuropas

Juniorprofessur (Uni)

Fachhochschulprofessur

W2/C3/C2-Professur (Uni)

W3/C4-Professur (Uni)
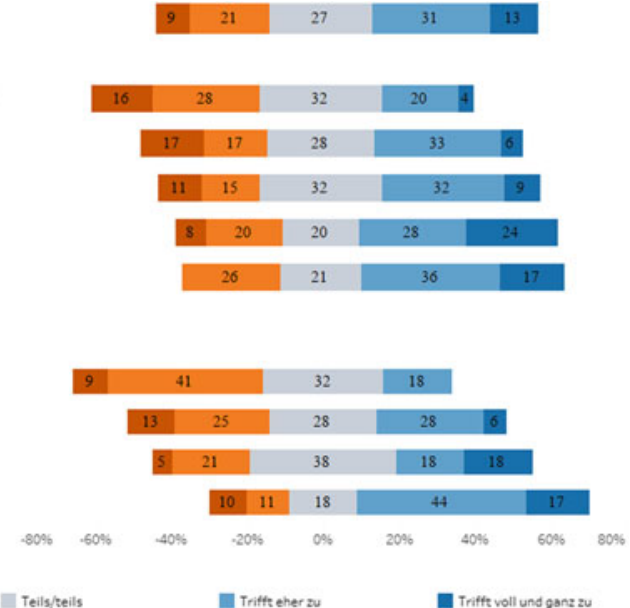

Abbildung 6.29 Soziodemographische Unterschiede hinsichtlich der Beurteilung des Items „genügend Rückmeldung über Leistungen in der Forschung“. (Quelle: MOBIL 2012/2013 (Gesamt: $\mathrm{N}=168$, Herkunftsregion: $\mathrm{N}=168(\mathrm{p}=0,082)$, Besoldungsgruppe: $\mathrm{N}=165$ ( $\mathrm{p}$ $=0,003), \mathrm{p}$-value basiert auf Kruskal-Wallis-Test)

Fast die Hälfte der Professoren beurteilt die Bezahlung als unangemessen (46 \%), während lediglich ca. ein Drittel mit der Bezahlung einverstanden ist. Nach regionaler Herkunft zeigen sich keine signifikanten Unterscheide. Allerdings wird sichtbar, dass über die Hälfte der Professoren aus den USA, Kanada und Australien unzufrieden ist mit der Bezahlung, was vermutlich in Verbindung mit den deutlich höheren Durchschnittsgehältern der Professoren in diesen Ländern 
steht (vgl. Abschnitt 2.3). Auffällig ist auch, dass sich keine signifikanten Unterschiede nach Besoldungs- und Fächergruppen ${ }^{21}$ zeigen. Demgegenüber beurteilen Early Migrants die Bezahlung deutlich kritischer als Professoren, die erst im Studium oder als Berufstätige nach Deutschland gekommen sind. Ein weiterer interessanter Unterschied betrifft die soziale Herkunft. Die Hälfte der Professoren aus nichtakademischen Elternhäusern beurteilt die Bezahlung als angemessen, während unter Professoren, die mindestens einen Elternteil mit Hochschulabschluss haben, weniger als ein Drittel dieser Meinung ist. Die Ergebnisse zeigen, dass anscheinend nicht allein die Höhe des Einkommens (Besoldungsgruppe) und die unterschiedlichen Verdienstmöglichkeiten außerhalb der Hochschule (Fächergruppe) für die Zufriedenheit mit dem Einkommen maßgeblich sind, sondern dass auch dem sozialen und familiären Umfeld als Referenzrahmen eine erhebliche Bedeutung zukommt (vgl. Abbildung 6.30).
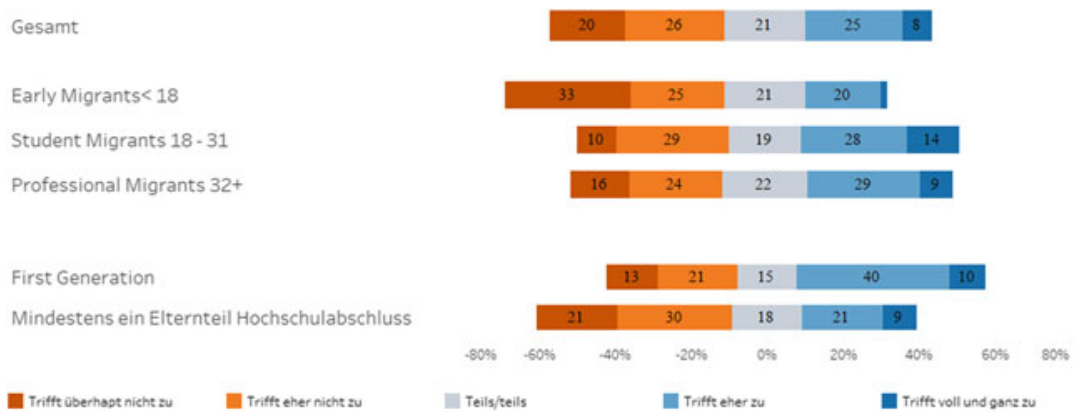

Abbildung 6.30 Soziodemographische Unterschiede hinsichtlich der Beurteilung des Items ,angemessene Bezahlung“. (Quelle: MOBIL 2012/2013 (Gesamt: N = 177, Migrationstypen: $\mathrm{N}=177(\mathrm{p}=0,004)$, Soziale Herkunft: $\mathrm{N}=155(\mathrm{p}=0,033)$, p-value basiert auf KruskalWallis-Test))

Die soziale Herkunft spielt auch bei der Beurteilung der Kooperation im Kollegium eine wichtige Rolle. Insgesamt wird die Kooperation von über der Hälfte der Professoren positiv bewertet. Bei Professoren der First Generation liegt der Anteil sogar bei über zwei Dritteln. Im Hinblick auf die in der Migrationsforschung häufig aufgeworfene Frage der Integration bleibt festzuhalten, dass der Großteil der

\footnotetext{
${ }^{21}$ Aufgrund der unterschiedlichen Gehaltszulagen bestehen hier insbesondere seit der Umstellung auf die W-Besoldung ebenfalls erhebliche Unterschiede.
} 
Professoren mit Migrationshintergrund auf gute Kooperationsstrukturen hinweist (vgl. Abbildung 6.31).

Gesamt

First Generation

Mindestens ein Elternteil Hochschulabschluss

Trifft uberhapt nicht zu

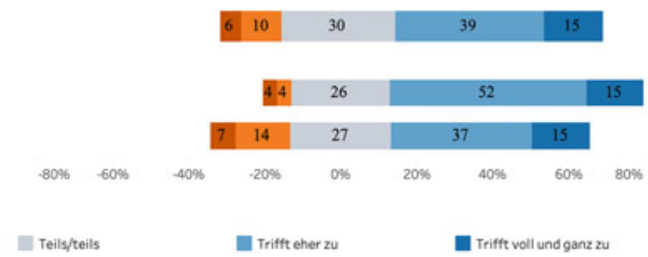

Abbildung 6.31 Soziodemographische Unterschiede hinsichtlich der Beurteilung des Items „gute Kooperation im Kollegium“. (Quelle: MOBIL 2012/2013 Gesamt N = 181, Soziale Herkunft: $\mathrm{N}=159$ ( $\mathrm{p}=0,10)$, p-value basiert auf Mann-Whitney-U-Test)

Die geringe institutionelle Anerkennung der Lehre wird von über der Hälfte der Professoren mit Migrationshintergrund kritisiert. Kritik wird in besonderem Maße von Juniorprofessoren und W2-/C3-/C2-Professoren geäußert. Die vorwiegend negative Beurteilung und der starke Zusammenhang mit der beruflichen Zufriedenheit deuten klar darauf hin, dass in diesem Bereich politischer Handlungsbedarf besteht. Dabei sollte berücksichtigt werden, dass der Großteil der Professoren mit Migrationshintergrund aus dem Ausland zugewandert ist und daher bereits Wissenschaftskulturen anderer Länder kennenlernen konnte. Dementsprechend könnte im Rahmen internationaler Vergleichsforschung untersucht werden, in welchen ausländischen Modellen die Lehre eine stärkere institutionelle Anerkennung erfährt und wie auf dieser Grundlage das bestehende Modell in Deutschland ggf. weiterentwickelt werden kann (vgl. Abbildung 6.32).

Zusammenfassend bleibt festzuhalten, dass sich die Beurteilung der Arbeitsbedingungen insbesondere zwischen den Besoldungsgruppen grundlegend unterscheidet. Insbesondere Juniorprofessoren und Fachschulprofessoren zeigen sich eine sehr kritische Perspektive auf die genannten Aspekte der Arbeitsbedingungen. Interessant wäre, in zukünftigen Studien herauszuarbeiten, inwieweit sich die Aspekte in ähnlicher Form auch für Juniorprofessoren und Fachhochschulprofessoren ohne Migrationshintergrund zeigen, also primär auf die Besoldungsgruppe bzw. Hochschulart zurückzuführen sind oder ob es ein Spezifikum von Professoren mit Migrationshintergrund darstellt. 


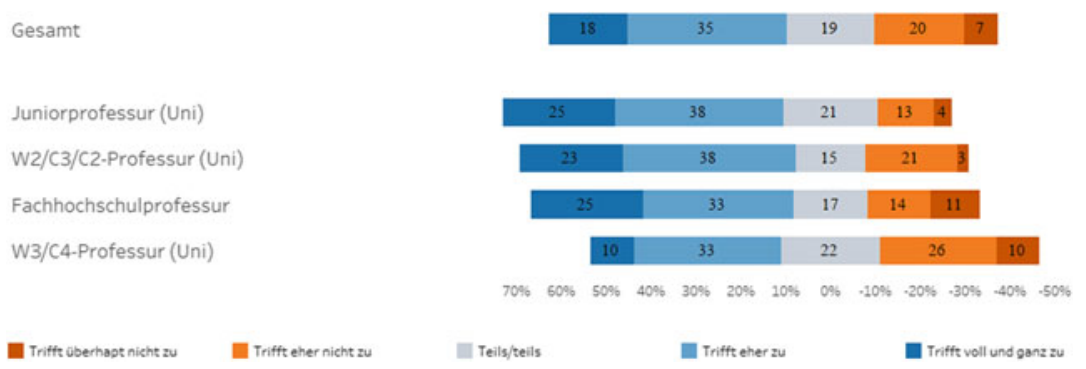

Abbildung 6.32 Soziodemographische Unterschiede hinsichtlich der Beurteilung des Items ,geringe institutionelle Anerkennung für Leistungen in der Lehre“. (Quelle: MOBIL 2012/2013 (Gesamt: $N=175$, Besoldungsgruppen: $N=172(p=0,049)$, p-value basiert auf Kruskal-Wallis-Test)

\subsection{Internationale Mobilität und internationale Aktivitäten}

Eine zentrale Bedeutung kommt der Frage zu, in welchem Maße Professoren mit Migrationshintergrund auf die Internationalisierung der Hochschulen Einfluss nehmen. Zunächst ist zu klären, was unter dem Begriff der Internationalisierung in diesem Zusammenhang genau zu verstehen ist. Wie in Abschnitt 3.3.1 bereits ausführlich dargelegt, wird in Anlehnung an Knight die Internationalisierung der Hochschule als "process of integrating an international, intercultural or global dimension into the purpose, functions or delivery of post-secondary education " (Knight 2004, S. 11) verstanden.

Um die Frage zu untersuchen, inwieweit Professoren mit Migrationshintergrund an Hochschulen eine internationale, interkulturelle oder globale Dimension einbringen, werden im Folgenden fünf Felder unterschieden:

1. Internationalität des Bildungs-/Berufsverlaufs

2. Internationale Publikationen

3. Berufliche Auslandstätigkeiten

4. Lehre und Forschung in nichtdeutschen Sprachen

5. Interkulturelle Aktivitäten

Die fünf Felder schließen unmittelbar an die im theoretischen Teil dargelegten Ausführungen zur Internationalisierung der Hochschulen an (vgl. Abschnitt 3.3 und 3.4). Bei der Internationalität des Bildungs- und Berufsverlauf geht es 
darum, die unterschiedlichen Formen grenzüberschreitender internationaler Mobilität über einen Index sichtbar zu machen. Internationale Publikationen, berufliche Auslandstätigkeiten und Forschung in nichtdeutschen Sprachen beziehen sich auf die international dimension nach Knight, das heißt es geht um internationale Beziehungen, Austausch und Sichtbarkeit, insbesondere im Feld der Forschung. Abschließend geht es bei der Lehre in nichtdeutschen Sprachen und bei interkulturellen Aktivitäten, die sich auf Betreuung und Austausch mit anderen Migranten im Professorenberuf beziehen, um die intercultural dimension. Das heißt es geht bei den beiden Indikatoren insbesondere darum, wie aktiv die Professoren mit Migrationshintergrund im Feld der Internationalization at home sind.

\subsubsection{Internationalität des Bildungs-/Berufsverlaufs}

Hinsichtlich des Bildungsverlaufs werden die zentralen Abschlüsse (Schule, Studium und Promotion) in den Blick genommen. Da weniger als die Hälfte der Professoren mit Migrationshintergrund eine abgeschlossene Habilitation vorweisen kann (vgl. Abschnitt 6.2.1) und die Habilitation international kaum verbreitet ist, wird dieser Abschluss in diesem Zusammenhang nicht berücksichtigt. Grundsätzlich bestehen zwei Möglichkeiten, internationale Erfahrung anhand der Bildungsabschlüsse zu operationalisieren. Zum einen lässt sich der Standpunkt vertreten, dass die Zahl im Ausland erworbener Bildungsabschlüsse einen geeigneten Indikator für internationale Erfahrung darstellt. Zum anderen ist es aber auch möglich, den Aspekt der internationalen Mobilität in den Fokus zu rücken und entsprechend die Zahl der Bildungsabschlüsse, die in unterschiedlichen Staaten erworben wurden, als Indikator heranzuziehen. Für die zweite Form der Operationalisierung spricht, dass hier stärker Berücksichtigung findet, ob Personen umfangreiche Erfahrungen in unterschiedlichen Bildungsoder Wissenschaftssystemen gesammelt haben. Zugleich bedingen sich beide Operationalisierungen bis zu einem gewissen Grad gegenseitig. Personen, die in unterschiedlichen Staaten Bildungsabschlüsse erworben haben, können logischerweise immer mindestens einen im Ausland erworbenen Bildungsabschluss vorweisen. Aus Sicht des Autors sollte der Aspekt der internationalen Mobilität bei der Operationalisierung internationaler Erfahrung stärker gewichtet werden. Nichtsdestotrotz sollte berücksichtigt werden, dass Professoren, die sämtliche Bildungsabschlüsse außerhalb Deutschlands, allerdings in ein und demselben Staat erworben haben, hinsichtlich des Bildungsverlaufs auf eine größere internationale Erfahrung zurückgreifen können als Professoren, die alle Bildungsabschlüsse in 
Deutschland erworben haben. Auf dieser Grundlage wurden anhand der Bildungsabschlüsse drei Gruppen gebildet, anhand derer sich die internationale Erfahrung einteilen lässt (vgl. Tabelle 6.10).

Die Ergebnisse zeigen, dass über ein Viertel der Professoren mit Migrationshintergrund sämtliche Bildungsabschlüsse in Deutschland erworben hat. Die größte Gruppe hat alle Bildungsabschlüsse im selben ausländischen Staat erworben, während knapp ein Drittel Bildungsabschlüsse aus unterschiedlichen Staaten vorweisen kann (vgl. Tabelle 6.10).

Tabelle 6.10 Internationale Erfahrung und Mobilität im Kontext der Bildungsabschlüsse

\begin{tabular}{l|l|l}
\hline & Index & Anteil (in \%) \\
\hline Alle Bildungsabschlüsse in Deutschland & 0 & 28 \\
\hline Alle Bildungsabschlüsse im selben ausländischen Staat & 1 & 42 \\
\hline Bildungsabschlüsse in unterschiedlichen Staaten & 2 & 30 \\
\hline
\end{tabular}

Quelle: MOBIL 2012/2013 N = 158

Während der zuvor genannte Indikator ausschließlich die Zahl der auswärtig erworbenen Bildungsabschlüsse berücksichtigt, soll im Folgenden in den Fokus gerückt werden, in welchem Maße während der Bildungsphasen Zeit im Ausland verbracht wurde. Diesbezüglich wurden die Professoren mit Migrationshintergrund im Rahmen der Erhebung gefragt, ob sie, abgesehen von den Staaten ihrer Bildungsabschlüsse, noch in weiteren Ländern zur Schule gegangen sind oder studiert haben. Knapp die Hälfte der Professoren (48\%) kann entsprechende internationale Mobilitätserfahrungen vorweisen.

In einem nächsten Schritt wurde die internationale berufliche Erfahrung, die vor der Berufung auf eine Professur in Deutschland gesammelt wurde, näher untersucht. Generell war ein Großteil der Professoren mit Migrationshintergrund (82\%) zuvor bereits beruflich im Ausland tätig. Davon waren ca. $8 \%$ in einem, ca. $34 \%$ in zwei, ca. $21 \%$ in drei und ca. $19 \%$ in vier oder mehr ausländischen Staaten beruflich tätig. Auf dieser Grundlage wurden im Hinblick auf die Indexbildung auch hier drei unterschiedliche Gruppen gebildet (vgl. Tabelle 6.11). Dabei ist zu berücksichtigen, dass keine Angaben über die Dauer der beruflichen Tätigkeit vorliegen. Es kann also durchaus sein, dass bspw. kurzzeitige Lehr- oder Forschungsaufenthalte auch als berufliche Tätigkeiten in dem jeweiligen Staat beschrieben wurden.

Auf der Grundlage der drei Indikatoren hinsichtlich der internationalen Erfahrung im Rahmen der Bildungsabschlüsse, der Schul- oder Studienzeiten in 
Tabelle 6.11 Berufliche Tätigkeiten in verschiedenen ausländischen Staaten

\begin{tabular}{l|l|l}
\hline & Additiver Index & Anteil (in \%) \\
\hline Keine berufliche Tätigkeit im Ausland & 0 & 18 \\
\hline $\begin{array}{l}\text { Berufliche Tätigkeit in einem oder zwei ausländischen } \\
\text { Staaten }\end{array}$ & 1 & 42 \\
\hline $\begin{array}{l}\text { Berufliche Tätigkeit in drei oder mehr ausländischen } \\
\text { Staaten }\end{array}$ & 2 & 40 \\
\hline
\end{tabular}

Quelle: MOBIL 2012/2013 N = 184

weiteren Staaten (Index $0=$ keine Zeiten in weiteren Staaten, $1=$ Schuloder Studienzeit in weiteren Staaten) sowie im Hinblick auf berufliche Tätigkeiten in verschiedenen ausländischen Staaten wurde ein additiver Index gebildet, der zwischen 0 (Bildungs-/Berufsverlaufs ausschließlich in Deutschland) und 5 (hochgradig internationaler Bildungs-/Berufsverlauf) variiert.

Im Folgenden werden diese in vier Gruppen zusammengefasst. Die erste Gruppe mit Indexwerten von 0 und 1 gibt an, dass entweder der Bildungs- oder der Berufsverlauf vollständig in Deutschland stattgefunden hat oder lediglich eine Phase zu einem gewissen Grad internationale Bezüge aufweist. Bei knapp einem Drittel der Professoren lässt sich von einem eher internationalen Bildungs- und Berufsverlauf sprechen, da hier entweder der Bildungs- oder der Berufsverlauf stark oder beide bis zu einem gewissen Grad international geprägt sind. Bei der dritten und größten Gruppe, die über ein Drittel der Professoren ausmacht, zeigt sich ein deutliches internationales Profil mit einem Indexwert von 3. Die vierte Gruppe mit einem Anteil von $22 \%$ der Professoren mit Migrationshintergrund verweist auf einen sehr oder hochgradig internationalen Bildungs- und Berufsverlauf mit Bildungsabschlüssen und beruflichen Tätigkeiten in einer Vielzahl von Ländern (Tabelle 6.12).

Professoren, die während oder nach Abschluss des Studiums nach Deutschland gekommen sind, können generell auf eine internationalere Bildungs- und Berufslaufbahn zurückblicken als Early Migrants. Erklären lässt sich dies unter anderem darüber, dass diese Gruppe mit höherer Wahrscheinlichkeit in verschiedenen Staaten Bildungsabschlüsse erworben hat bzw. einer Berufstätigkeit nachgegangen ist. Interessant ist in diesem Zusammenhang, dass sich die Bildungs- und Berufslaufbahnen von Early Migrants und Professoren der zweiten Generation ebenfalls als eher international beschreiben lassen (vgl. Abbildung 6.33). Hier zeigen sich Parallelen zur CEWS-Erhebung (Löther 2012a), in der darauf hingewiesen wird, dass 
Tabelle 6.12 Index zur Internationalität des Bildungs-/Berufsverlaufs

\begin{tabular}{l|l|l}
\hline Internationalität des Bildungs-/Berufsverlaufs & Additiver Index & Anteil (in \%) \\
\hline Ausschließlich in Deutschland & 0 & 3 \\
\hline Kaum international & 1 & 10 \\
\hline Eher international & 2 & 30 \\
\hline International & 3 & 35 \\
\hline Sehr international & 4 & 14 \\
\hline Hochgradig international & 5 & 8
\end{tabular}

Quelle: MOBIL 2012/2013 N = 148

Wissenschaftler der zweiten Zuwanderergeneration häufig international mobiler sind als Professoren ohne Migrationshintergrund (vgl. Abschnitt 4.3.2).

Auffällige Unterschiede sind zudem zwischen den Fächergruppen zu beobachten. So sind Professoren aus den Naturwissenschaften und in den Geisteswissenschaften in besonderem Maße international mobil, während der Grad internationaler Mobilität in den Ingenieur- und Lebenswissenschaften deutlich geringer ausfällt (vgl. Abbildung 6.33). Hier werden Parallelen zu bibliometrischen Analysen sichtbar, die ebenfalls zu dem Ergebnis kommen, dass bei Wissenschaftlern in den Natur- und Geisteswissenschaften in besonderem Maße internationale Mobilitätsbiographien stattfinden (vgl. DAAD 2016 und Abschnitt 4.1).

Deutlich internationalere Biographien können auch Professoren aus akademischen Elternhäusern im Vergleich zu Professoren der First Generation vorweisen (vgl. Abbildung 6.33). Die größten Unterschiede zeigen sich innerhalb der Gruppe der Early Migrants. Hier variiert der Index zwischen 1,5 bei Professoren der First Generation und 2,4 bei Professoren aus akademischen Elternhäusern. Ein möglicher Grund für die geringere internationale Mobilität könnte in diesem Zusammenhang auch auf das Fehlen entsprechender finanzieller Ressourcen zurückzuführen sein. Zugleich zeigt sich, dass der aus der Studierendenforschung bekannte Befund (Lörz et al. 2016), dass ein nichtakademisches Elternhaus die Wahrscheinlichkeit für internationale Mobilität im Studium deutlich verringert (vgl. Abschnitt 4.3.3), ebenfalls hinsichtlich der gesamten Bildungs- und Berufsverläufe der First Generation Professoren mit Migrationshintergrund gilt. 
Gesamt

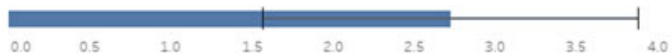

Zweite Generation

Ober 20 Jahre in Deutschl. Zwischen 11-20 Jahren

Max. 10 Jahre in Deutschl.

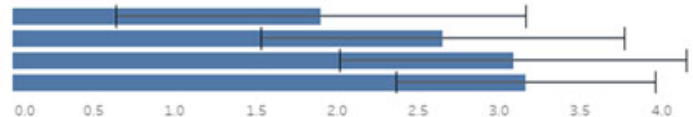

Early Migrants $<18$ Student Migrants $18-31$

Professional Migrants $32+$
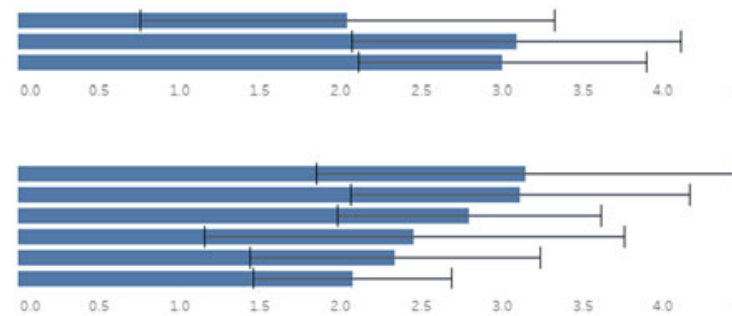

Akademisches Elternhaus Nicht akad. Elternhaus

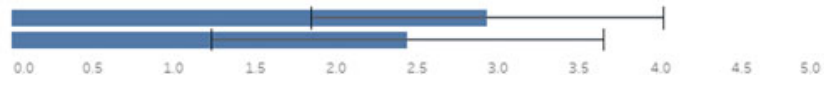

$\longmapsto$ Standardabweichung; $0=$ keine internationale Bildungs-/Berufslaufbahn, $5=$ hochgradig internationale Bildungs/Berufslaufbahn (vgl. Tabelle 15).

Abbildung 6.33 Gruppenunterschiede hinsichtlich der Internationalität der Bildungs/Berufslaufbahn. (Quelle: MOBIL 2012/2013, Gesamt: N = 148 Dauer in Deutschland: N = 148 (p1<0,001; p2<0,001) Migrationstypen: $N=148$ (p1<0,001, p2<0,001) Fächergruppen: $\mathrm{N}=142$ (p1 = 0,01, p2 =0,006) Soziale Herkunft $\mathrm{N}=131(\mathrm{p} 1=0,04, \mathrm{p} 2=0,04)$; p1-value basiert auf einfaktorieller ANOVA; p2-value basiert auf Mann-Whitney-U-Test und Kruskal-Wallis-Test)

\subsubsection{Internationale Publikationen}

Hinsichtlich der Internationalität von Professoren mit Migrationshintergrund sind ferner die internationalen Publikationen der letzten Jahre näher zu betrachten. Publikationen in einer anderen Sprache als Deutsch und Publikationen außerhalb Deutschlands können jeweils fast $90 \%$ der Professoren vorweisen. Gemeinsame Publikationen mit ausländischen Kollegen sind bei knapp zwei Dritteln der Befragten zu verzeichnen (vgl. Abbildung 6.34).

Ausgehend von diesen drei internationalen Publikationsformen lässt sich ein Index bilden, der zwischen 0 (keine internationale Publikation) und 3 (alle drei internationalen Publikationsformen) variiert. 
In einer anderen Sprache als Deutsch

Außerhalb Deutschlands

Mit auslandischen Kollegen

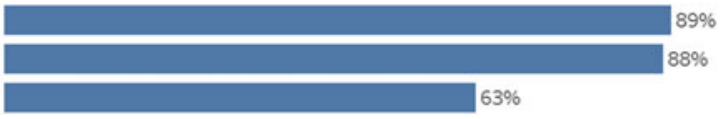

Abbildung 6.34 Formen internationaler Publikationen. (Quelle: MOBIL 2012/2013 N = 188)

Gesamt
Jungere Generation< $=44$
Mittlere Generation $45-53$
Altere Generation $54+$

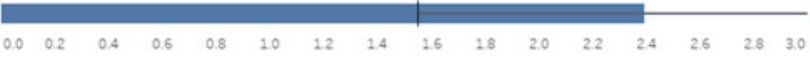
Lebenswiss.

Geisteswiss.

Sozialwissenschaften

Kunstwissenschaften

Ingenieurwiss.

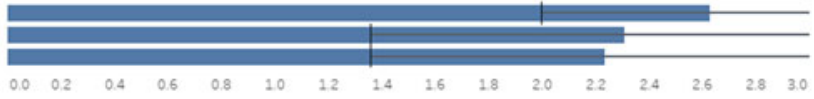

Juniorprofessur (Uni) W3/C4-Professur (Uni) W2/C3/C2-Professur (Uni) Fachhochschulprofessur
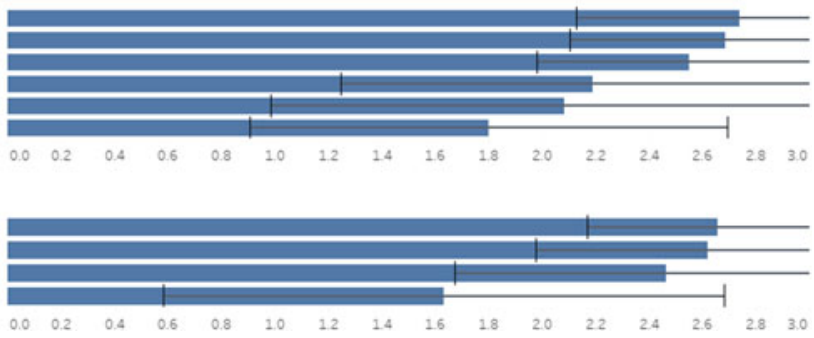

$\longmapsto$ Standardabweichung; $0=$ keine internationale Publikationsform; 3 = alle drei Formen internationaler Publikationen.

Abbildung 6.35 Gruppenunterschiede bei internationalen Publikationen. (Quelle: MOBIL 2012/2013, Gesamt: $N=188$ Alter: $N=188$ (p1 $=0,02, p 2<0,02)$ Fächergruppen: $N$ $=182(\mathrm{p} 1<0,001, \mathrm{p} 2<0,001)$ Besoldungsgruppen $\mathrm{N}=184(\mathrm{p} 1<0,001, \mathrm{p} 2<0,001) ; \mathrm{p} 1-$ value basiert auf einfaktorieller ANOVA; p2-value basiert auf Mann-Whitney-U-Test und Kruskal-Wallis-Test)

Am häufigsten können jüngere Professoren und Juniorprofessoren unterschiedliche Formen internationaler Publikationen vorweisen. Auch Professoren aus den Natur-, Lebens-, und Geisteswissenschaften zeichnen sich durch ein stark international geprägtes Publikationsprofil aus. Demgegenüber fällt der entsprechende Anteil in den Ingenieurwissenschaften und an Fachhochschulen deutlich geringer aus. Erwähnenswert ist zudem, dass der Zeitpunkt der Zuwanderung keinen Einfluss auf die internationale Publikationstätigkeit hat (vgl. Abbildung 6.35). 


\subsubsection{Berufliche Auslandstätigkeiten}

Als dritte Dimension internationaler Mobilität werden berufliche Auslandstätigkeiten der letzten Jahre näher untersucht. Über drei Viertel der Professoren mit Migrationshintergrund waren in den letzten Jahren anlässlich von Konferenzen, Workshops, Gastvorträgen, Ausstellungen oder künstlerischen Auftritten im Ausland. Ein Drittel der Befragten ist dabei Aktivitäten im Rahmen internationaler Hochschulkooperationen nachgegangen. Der entsprechende Anteil in Bezug auf Forschungsaufenthalte fällt noch etwas höher aus, während er in Bezug auf Lehrtätigkeiten etwas darunter liegt. Jeder fünfte Professor mit Migrationshintergrund hatte in den letzten Jahren eine Gastprofessur im Ausland inne (vgl. Abbildung 6.36).
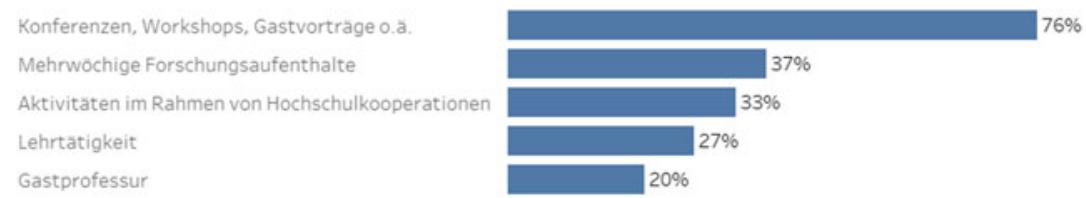

Abbildung 6.36 Berufliche Auslandstätigkeit in den letzten Jahren. (Quelle: MOBIL $2012 / 2013 \mathrm{~N}=183$ )

Hinsichtlich der unterschiedlichen Auslandstätigkeiten wurde ebenfalls ein Index erstellt, der zwischen 0 (keine berufliche Auslandstätigkeit) und 5 (alle fünf Formen beruflicher Auslandstätigkeiten) variiert. Während die jüngere Generation und Juniorprofessoren im Bereich internationaler Publikationen am aktivsten waren, fällt der Index für die beiden Gruppen bei Auslandstätigkeiten am geringsten aus. Auch Professoren aus den Sozial- und Lebenswissenschaften sind in den letzten Jahren deutlich seltener Auslandstätigkeiten nachgegangen. Demgegenüber zeigt sich bei Professoren der Besoldungsgruppe W3/C4, bei Professoren aus den Geisteswissenschaften sowie bei Professoren der älteren Generation ein deutlich überdurchschnittlicher Indexwert. Zudem erreichen männliche Professoren einen höheren Wert $(2,07)$ als ihre Kolleginnen $(1,61)$ (vgl. Abbildung 6.37).

\subsubsection{Nichtdeutsche Sprachen in Forschung und Lehre}

Die Verwendung des Englischen in Lehre und Forschung hat sich unter Professoren mit Migrationshintergrund fest etabliert. So benutzt in der Lehre knapp 


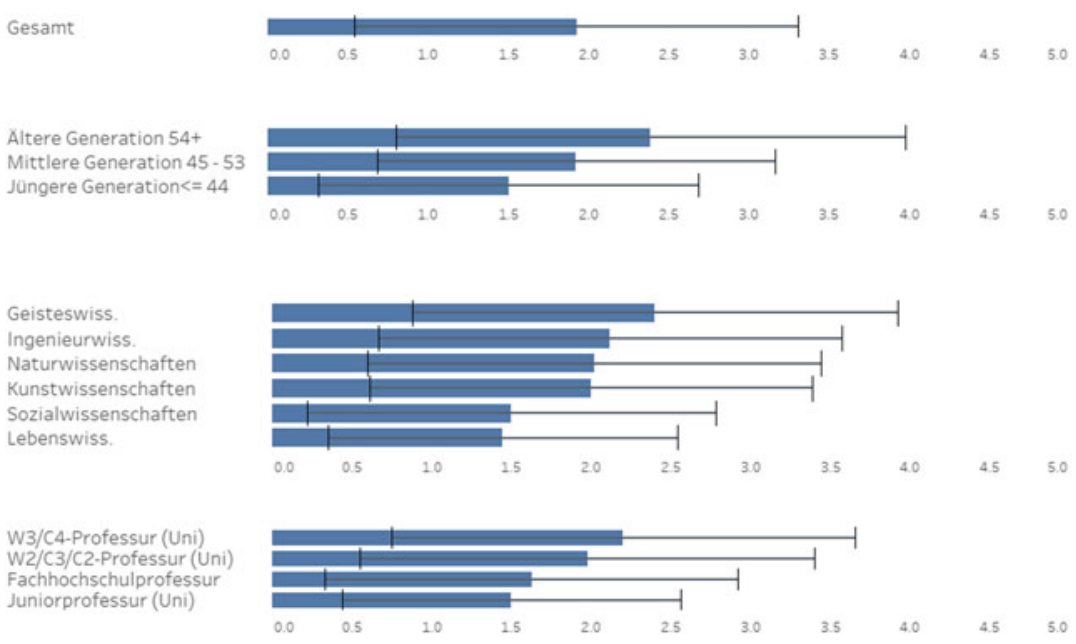

$\longmapsto$ Standardabweichung; $0=$ keine beruflichen Auslandstätigkeiten ; $5=$ alle fünf Formen beruflicher Auslandstätigkeiten.

Abbildung 6.37 Gruppenunterschiede bei beruflichen Auslandstätigkeiten. (Quelle: MOBIL 2012/2013, Gesamt: $\mathrm{N}=183$ Alter: $\mathrm{N}=183$ (p1 = 0,02, $\mathrm{p} 2=0,005)$ Fächergruppen: $\mathrm{N}=177(\mathrm{p} 1=0,076, \mathrm{p} 2=0,073)$ Besoldungsgruppen $\mathrm{N}=181(\mathrm{p} 1=0,065, \mathrm{p} 2=0,095)$; p1-value basiert auf Einfaktorieller ANOVA; p2-value basiert auf Mann-Whitney-U-Test und Kruskal-Wallis-Test)

die Hälfte häufig die englische Sprache, während in der Forschung der entsprechende Anteil sogar bei über $80 \%$ liegt. Die Relevanz des Englischen als lingua franca der Wissenschaft zeigt sich insbesondere im Vergleich zur Bedeutung von weiteren nichtdeutschen Sprachen in Forschung und Lehre. So werden in der Lehre andere nichtdeutsche Sprachen lediglich von ca. jedem achten Professor mit Migrationshintergrund verwendet. Etwas höher fällt dieser Anteil allerdings im Bereich der Forschung aus, wo er mit etwas über einem Viertel aber auch deutlich hinter der Verwendung der englischen Sprache zurückbleibt (vgl. Abbildung 6.38).

Rostan, Finkelstein und Huang (2014) verweisen darauf, dass Professoren in den Soft-Disciplines ${ }^{22}$ im Rahmen der CAP-Erhebung deutlich häufiger auf englischsprachige Lehrveranstaltungen verweisen als Professoren aus den

\footnotetext{
${ }^{22}$ Im Rahmen der Fächergruppeneinteilung der vorliegenden Arbeit fallen hierunter Sozial/Verhaltenswissenschaften, Geisteswissenschaften und Kunst/Kunstwissenschaften.
} 
Hard-Disciplines $^{23}$ (vgl. Abschnitt 4.3.4). Auch bei den Professoren mit Migrationshintergrund zeigt sich, dass über die Hälfte $(55 \%)$ der Professoren aus den Soft-Disciplines häufig die englische Sprache in der Lehre verwendet, während der Anteil in den Hard-Disciplines bei unter $40 \%$ liegt. Zugleich führen Rostan, Finkelstein und Huang (2014) an, dass demgegenüber in den Hard-Disciplines Englisch häufiger in der Forschung verwendet werde. Bei den Professoren mit Migrationshintergrund zeigen sich diesbezüglich jedoch keine Unterschiede.

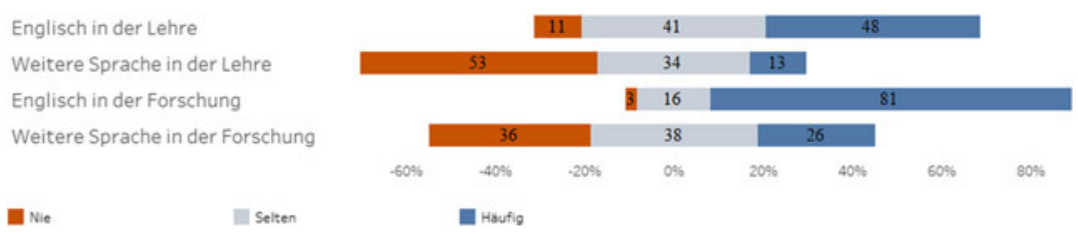

Abbildung 6.38 Nichtdeutsche Sprachen in Forschung und Lehre. (Quelle: MOBIL 2012/2013 (Item 1: $\mathrm{N}=196$, Item 2: $\mathrm{N}=143$, Item 3: 188, Item 4: 141))

Um zu erforschen, inwieweit unterschiedliche Professorengruppen mit Migrationshintergrund an den Hochschulen nichtdeutsche Sprachen einbringen, wird im Folgenden die Verwendung des Englischen sowie weiterer Sprachen für Lehre und Forschung gemeinsam betrachtet. Demnach verwenden Professoren mit Migrationshintergrund zu $50 \%$ häufig nichtdeutsche Sprachen in der Lehre und zu $84 \%$ in der Forschung. Auf der Grundlage dieser beiden Variablen wurde im nächsten Schritt ein Mittelwertindex gebildet, der auf einer Skala von 1 (nie) bis 3 (häufig) die Verwendung nichtdeutscher Sprachen in Forschung und Lehre veranschaulicht.

Unter allen Professoren liegt der Index bei 2,6. Einen der höchsten Indexwerte erreichen Professoren aus angelsächsischen Ländern. Da die zentrale nichtdeutsche Sprache in Forschung und Lehre die englische ist, ergibt sich für diese Gruppe englischer Muttersprachler ein besonderer Vorteil. Kaum einen Einfluss hat hingegen der Zuwanderungszeitpunkt. Der Index liegt für die zweite Einwanderergeneration sogar leicht über dem Durchschnitt. Besonders häufig verwenden zudem die jüngere Generation und insbesondere Juniorprofessoren

\footnotetext{
${ }^{23}$ Im Rahmen der Fächergruppeneinteilung der vorliegenden Arbeit fallen hierunter Naturwissenschaften, Ingenieurwissenschaften und Lebenswissenschaften.
} 
nichtdeutsche Sprachen in Forschung und Lehre. Auch in den Natur- und Sozial/Verhaltenswissenschaften werden besonders häufig nichtdeutsche Sprachen verwendet, während der entsprechende Anteil in den Ingenieurwissenschaften mit Abstand am geringsten ausfällt (vgl. Abbildung 6.39).

Gesamt

Angelsachsiche Lander* Westeuropa*:

Lateinamerika, Asien, Afrika

Osterreich und Schweiz

Osteuropa

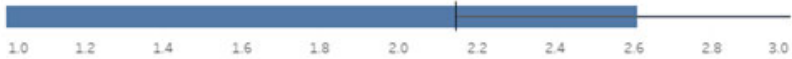

Jünere Generation $<=44$

Mittlere Generation 45 - 53

Altere Generation 54+
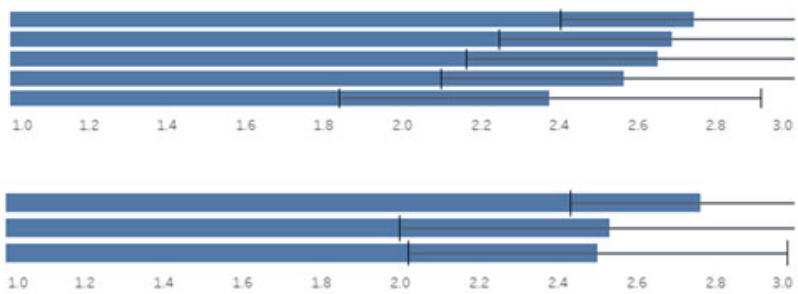

Naturwissenschaften

Sozialwissenschaften

Geisteswiss.

Kunstwissenschaften

Lebenswiss.

Ingenieurwiss.

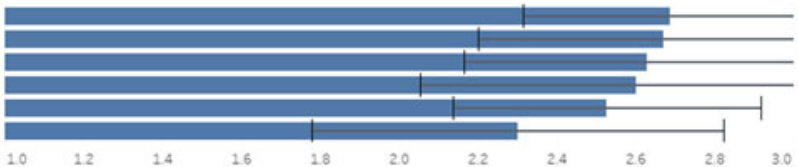

Juniorprofessur (Uni)

W3/C4-Professur (Uni)

W2/C $3 /$ C2-Professur (Uni)

Fachhochschulprofessur

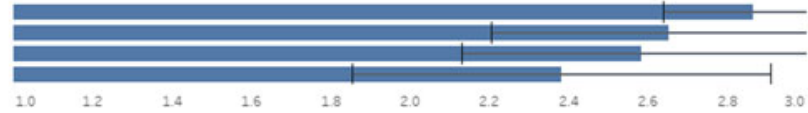

Standardabweichung; 1 = nie, 2 = selten, 3 = häufig. *USA, Kanada, Australien; **ohne Österreich und Schweiz.

Abbildung 6.39 Gruppenunterschiede hinsichtlich der Verwendung nichtdeutscher Sprachen in Forschung/Lehre. (Quelle: MOBIL 2012/2013, Gesamt: N = 198 Regionale Herkunft: $\mathrm{N}=197(\mathrm{p} 1=0,016, \mathrm{p} 2=0,023)$ Alter: $\mathrm{N}=197(\mathrm{p} 1=0,003, \mathrm{p} 2=0,007)$ Fächergruppen: $\mathrm{N}=190(\mathrm{p} 1=0,043, \mathrm{p} 2=0,038)$ Besoldungsgruppen $\mathrm{N}=193(\mathrm{p} 1<0,001, \mathrm{p} 2<0,001)$; p1-value basiert auf einfaktorieller ANOVA; p2-value basiert auf Mann-Whitney-U-Test und Kruskal-Wallis-Test) 


\subsubsection{Interkulturelle Aktivitäten}

Im Folgenden sollen die interkulturellen Aktivitäten der Professoren mit Migrationshintergrund näher untersucht werden. Als interkulturelle Aktivitäten werden dabei Interaktionen mit Menschen mit Migrationshintergrund verstanden. Grundsätzlich ließe sich auch die Interaktion zwischen Professoren mit Migrationshintergrund und Menschen ohne Migrationshintergrund als interkulturell bezeichnen. An dieser Stelle wird aber zunächst ausschließlich die Interaktion zu anderen Menschen mit Migrationshintergrund unter dem Stichwort Interkulturalität untersucht, da ein solches Verständnis auch der gebräuchlichen Verwendung im gesellschaftlichen Diskurs und Wissenschaft entspricht.

Skachkova (2007) zeigt auf der Grundlage der Befragung von Professorinnen mit Migrationshintergrund an großen Forschungsuniversitäten in den USA, dass die Professorinnen für migrant students häufig ein wichtiges Role Model darstellen (vgl. Abschnitt 4.3.8). Auch bei den befragten Professoren mit Migrationshintergrund in Berlin und Hessen zeigt sich eine sehr enge Zusammenarbeit sowohl mit Studierenden als auch mit Mitarbeitern mit Migrationshintergrund. Fast die Hälfte der Professoren betreut regelmäßig Abschlussarbeiten von Studierenden mit Migrationshintergrund. Ein Drittel der Befragten beschäftigt zur Hälfte oder überwiegend Mitarbeiter mit Migrationshintergrund. Über $80 \%$ haben regelmäßig beruflichen Kontakt zu Menschen mit Migrationshintergrund in Deutschland. ${ }^{24}$ Die Ergebnisse zeigen, dass der berufliche Alltag von Professoren mit Migrationshintergrund in hohem Maße durch interkulturelle Kontakte geprägt ist (vgl. Abbildung 6.40).

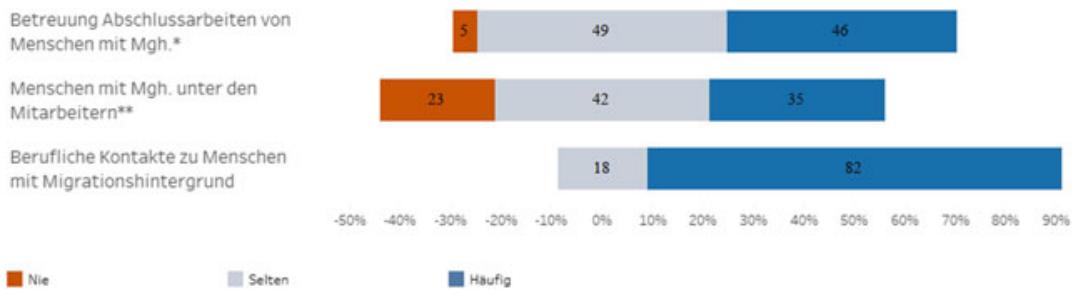

Abbildung 6.40 Interkulturelle Aktivitäten in Deutschland. (Quelle: MOBIL 2012/2013 (Item 1: $\mathrm{N}=182$, Item 2: $\mathrm{N}=175$, Item 3: 175))

\footnotetext{
${ }^{24}$ Variable wurde gebildet auf der Grundlage der zwei Items häufige berufliche Kontakte in Deutschland mit a) Menschen aus dem Herkunftsland und b) Menschen aus weiteren Ländern.
} 
Anhand der drei dargestellten Aspekte wurde wiederum ein Index gebildet, um Gruppenunterschiede systematisch abzubilden. Die berufliche Interaktion zu Menschen mit Migrationshintergrund wird auf einer Skala von 1 (keine Interaktion) bis 3 (häufige Interaktion mit unterschiedlichen Gruppen) dargestellt. Auffällige Unterschiede zeigen sich lediglich hinsichtlich der verschiedenen Fächerund Besoldungsgruppen. Professoren aus den Geistes- und Naturwissenschaften pflegen besonders häufig interkulturelle Kontakte, während der Anteil in den Ingenieurwissenschaften deutlich geringer ausfällt. Auch für Professoren an Universitäten fällt der Index deutlich höher aus als für Fachhochschulprofessoren (vgl. Abbildung 6.41).

Gesamt

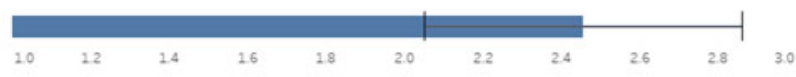

Geisteswiss.

Naturwissenschaften

Kunstwissenschaften

Lebenswiss.

Sozialwissenschaften

Ingenieurwiss.

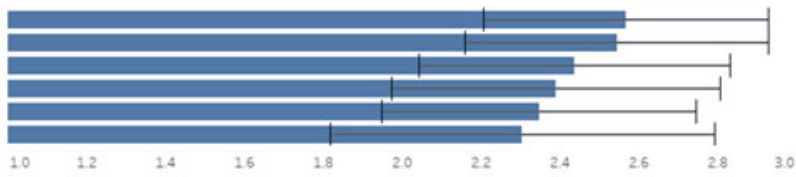

W3/C4-Professur (Uni)
W2/C3/C2-Professur (Uni)
Juniorprofessur (Uni)

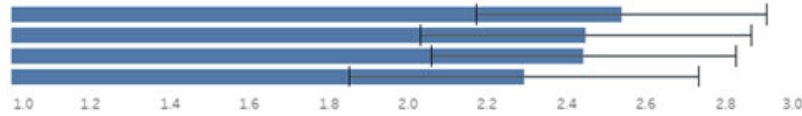

Fachhochschulprofessur

$\longmapsto$ Standardabweichung; $1=$ nie, $2=$ selten, 3 = häufig.

Abbildung 6.41 Unterschiede hinsichtlich interkultureller Aktivitäten in Deutschland. (Quelle: MOBIL 2012/2013 Gesamt: $\mathrm{N}=182$ Fächergruppen: $\mathrm{N}=176$ (p1 = 0,069, p2 = $0,10)$ Besoldungsgruppen $\mathrm{N}=179(\mathrm{p} 1=0,021, \mathrm{p} 2=0,03)$; $\mathrm{p} 1$-value basiert auf einfaktorieller ANOVA; p2-value basiert auf Mann-Whitney-U-Test und Kruskal-Wallis-Test)

\subsubsection{Zusammenhänge internationaler Aktivitäten}

Generell ist zunächst festzuhalten, dass sich in allen fünf untersuchten Dimensionen ein hoher Grad an Internationalität bei Professoren mit Migrationshintergrund zeigt. Abschließend soll untersucht werden, inwieweit sich die unterschiedlichen Dimensionen gegenseitig beeinflussen, das heißt inwiefern bspw. eine 
internationalere Bildungs-/Berufslaufbahn positiv mit internationalen Publikationstätigkeiten korreliert.

Dafür wurden zunächst zwei Korrelationskoeffizienten berechnet. Erstens wurde der parametrische Korrelationskoeffizient nach Pearson (r1) dargestellt. Zweitens wurde aufgrund der nicht durchgehend vorliegenden Normalverteilung der nichtparametrische Korrelationskoeffizient nach Spearman (r2) berechnet. Als Orientierung zur Einordnung der Stärke der Korrelation wird auf die Klassifikation nach Cohen zurückgegriffen $(r=0,1-0,29$ schwacher Zusammenhang; $r=$ 0,3-0,49 mittlerer Zusammenhang; $r=0,5-1$ starker Zusammenhang) (Pallant 2013, S. 138). Das Signifikanzniveau liegt auch hier bei $90 \%$. Nach dieser Systematik ergeben sich drei Gruppen: nichtsignifikante Korrelationen, signifikante Korrelationen mit schwachem Zusammenhang und signifikante Korrelationen mit mittlerem Zusammenhang (vgl. Abbildung 6.42).

Zwischen der Ausübung beruflicher Auslandstätigkeiten und der Verwendung nichtdeutscher Sprachen in Forschung und Lehre besteht kein signifikanter Zusammenhang. Ansonsten sind zwischen allen Indexen signifikante Korrelationen zu verzeichnen. So korreliert beispielsweise eine internationale Bildungs/Berufslaufbahn positiv mit internationalen Publikationen, beruflichen Auslandstätigkeiten, der Verwendung nichtdeutscher Sprachen in Forschung und Lehre sowie mit interkulturellen Aktivitäten (vgl. Abbildung 6.42). Der positive Einfluss der internationalen Bildungs- und Berufsverläufe auf weitere internationale Aktivitäten wurde auch bereits in anderen Hochschullehrerbefragungen nachgewiesen und gilt in ähnlicher Form auch für Professoren ohne Migrationshintergrund (vgl. Abschnitt 4.3.3 und 4.3.4).

Drei spezifische Zusammenhänge erreichen einen mittleren bis hohen Korrelationskoeffizienten. Weniger überraschend ist die hohe Korrelation zwischen internationalen Publikationen und nichtdeutschen Sprachen in Forschung und Lehre. So ist die Wahrscheinlichkeit recht hoch, dass Professoren, die im Ausland und in nichtdeutschen Sprachen publizieren, gleichzeitig auch in besonderem Maße nichtdeutsche Sprachen speziell in der Forschung verwenden. Interessant ist, dass sowohl internationale Publikationen als auch die Verwendung nichtdeutscher Sprachen in Forschung und Lehre eine vergleichsweise hohe Korrelation mit interkulturellen Aktivitäten aufweisen (vgl. Abbildung 6.42). Ein möglicher Erklärungsansatz kann in der Relevanz der Sprache für den interkulturellen Austausch liegen. Möglicherweise fällt es Professoren mit Migrationshintergrund, die häufig in nichtdeutschen Sprachen tätig sind, leichter, beruflichen Kontakt zu nicht-deutschsprachigen Menschen mit Migrationshintergrund in Deutschland aufzubauen. Zugleich kann es sein, dass Menschen mit Migrationshintergrund 


\begin{tabular}{|c|c|c|c|c|c|}
\hline Indexe & $\begin{array}{l}\text { Internationalität } \\
\text { der Bildungs- } \\
\text { /Berufslaufbahn }\end{array}$ & $\begin{array}{l}\text { Internationale } \\
\text { Publikationen }\end{array}$ & $\begin{array}{l}\text { Auslands- } \\
\text { tätigkeiten }\end{array}$ & $\begin{array}{c}\text { Nichtdeutsche } \\
\text { Sprache Lehre } \\
\text { und } \\
\text { Forschung }\end{array}$ & $\begin{array}{c}\text { Interkulturelle } \\
\text { Aktivitäten }\end{array}$ \\
\hline $\begin{array}{l}\text { Internationalität } \\
\text { der Bildungs- } \\
\text { /Berufslaufbahn }\end{array}$ & & $\begin{array}{c}\mathrm{r} 1=0,18 \\
\mathrm{p} 1=0,031 \\
(\mathrm{~N}=145)\end{array}$ & $\begin{array}{c}\mathrm{r} 1=0,169 \\
\mathrm{pl}=0,04 \\
(\mathrm{~N}=148)\end{array}$ & $\begin{array}{r}\mathrm{r} 1=0,209 \\
\mathrm{p} 1=0,011 \\
(\mathrm{~N}=148)\end{array}$ & $\begin{array}{c}\mathrm{r} 1=0,217 \\
\mathrm{p} 1=0,008 \\
(\mathrm{~N}=147)\end{array}$ \\
\hline $\begin{array}{l}\text { Internationale } \\
\text { Publikationen }\end{array}$ & $\begin{array}{r}\mathrm{r} 2=0,165 \\
\mathrm{p} 2=0,047 \\
(\mathrm{~N}=145)\end{array}$ & & $\begin{array}{r}\mathrm{r} 1=0,186 \\
\mathrm{p} 1=0,014 \\
(\mathrm{~N}=175)\end{array}$ & $\begin{array}{l}\mathrm{r} 1=0,366 \\
\mathrm{p} 1<0,001 \\
(\mathrm{~N}=188)\end{array}$ & $\begin{array}{r}\mathrm{r} 1=0,381 \\
\mathrm{p} 1<0,001 \\
(\mathrm{~N}=174)\end{array}$ \\
\hline $\begin{array}{l}\text { Auslands- } \\
\text { tätigkeiten }\end{array}$ & $\begin{array}{c}\mathrm{r} 2=0,151 \\
\mathrm{p} 2=0,067 \\
(\mathrm{~N}=148)\end{array}$ & $\begin{array}{c}\mathrm{r} 2=0,156 \\
\mathrm{p} 2=0,040 \\
(\mathrm{~N}=175)\end{array}$ & & $\begin{array}{l}\mathrm{r} 1=0,112 \\
\mathrm{p} 1=0,13 \\
(\mathrm{~N}=183)\end{array}$ & $\begin{array}{r}\mathrm{r} 1=0,208 \\
\mathrm{p} 1=0,005 \\
(\mathrm{~N}=182)\end{array}$ \\
\hline $\begin{array}{l}\text { Nichtdeutsche } \\
\text { Sprache Lehre } \\
\text { und Forschung }\end{array}$ & $\begin{array}{l}\mathrm{r} 2=0,185 \\
\mathrm{p} 2=0,025 \\
(\mathrm{~N}=148)\end{array}$ & $\begin{array}{l}\mathrm{r} 2=0,278 \\
\mathrm{p} 2<0,001 \\
(\mathrm{~N}=188)\end{array}$ & $\begin{array}{r}\mathrm{r} 2=0,109 \\
\mathrm{p} 2=0,141 \\
(\mathrm{~N}=183)\end{array}$ & & $\begin{array}{c}\mathrm{r} 1=0,412 \\
\mathrm{p} 1<0,001 \\
(\mathrm{~N}=182)\end{array}$ \\
\hline $\begin{array}{l}\text { Interkulturelle } \\
\text { Aktivitäten }\end{array}$ & $\begin{array}{c}\mathrm{r} 2=0,198 \\
\mathrm{p} 2=0,016 \\
(\mathrm{~N}=147)\end{array}$ & $\begin{array}{l}\mathrm{r} 2=0,386 \\
\mathrm{p} 2<0,001 \\
(\mathrm{~N}=174)\end{array}$ & $\begin{array}{r}\mathrm{r} 2=0,187 \\
\mathrm{p} 2=0,011 \\
(\mathrm{~N}=182)\end{array}$ & $\begin{array}{c}\mathrm{r} 2=0,386 \\
\mathrm{p} 2<0,001 \\
(\mathrm{~N}=182)\end{array}$ & \\
\hline $\begin{array}{c}\mathrm{r}=0,3-0,49 \\
\text { (mittlerer } \\
\text { Zusammenhang) }\end{array}$ & & $\begin{array}{l}\mathrm{r}=0,1-0,29 \\
\text { (schwacher } \\
\text { sammenhang) }\end{array}$ & & $\begin{array}{l}\text { a signifikanter } \\
\text { sammenhang }\end{array}$ & \\
\hline
\end{tabular}

Abbildung 6.42 Korrelationen zwischen den Indexen der Internationalität. (Quelle: MOBIL 2012/2013; r1/p1 basiert auf dem Korrelationsverfahren nach Pearson, r2/p2 basiert auf dem nichtparametrischen Verfahren von Spearman-Rho)

gezielt den Kontakt zu der spezifischen Professorengruppe möglicherweise aus dem gleichen Herkunftsland suchen.

\subsection{Zuwanderungsgeschichte, familiäre Situation und Zukunftspläne}

Das siebte Kapitel gliedert sich in fünf Teile. Im ersten Schritt werden Gründe und Kontext der Zuwanderung näher erläutert. Im Anschluss wird die Partnerschaft im Kontext der Migration und hinsichtlich der aktuellen Situation näher betrachtet. Darauf aufbauend wird die Frage der Vereinbarkeit von beruflicher Tätigkeit und Elternschaft für Professoren mit Migrationshintergrund untersucht. Die letzten beide Teile beschäftigen sich mit der Zukunftsplanung der Professoren mit 
Migrationshintergrund. Im vierten Teil werden die beruflichen Bleibeabsichten ausführlich analysiert, bevor im letzten Teil die nachberufliche Zukunftsplanung in den Mittelpunkt rückt.

\subsubsection{Gründe und Kontext der Zuwanderung}

An dieser Stelle ist zunächst der Kontext der Zuwanderung von Professoren mit Migrationshintergrund näher in den Blick zu nehmen. In einem ersten Schritt werden dabei die im Rahmen der Migrationsentscheidung relevanten Pro- und Kontra-Argumente für die Zuwanderung nach Deutschland betrachtet ${ }^{25}$. Das Modell orientiert sich dabei an funktionalistischen Ansätzen in der Migrationsforschung (vgl. Abschnitt 3.2.1.1). Die erhobenen Pro- und Kontraargumente wurden in Anlehnung an den aktuellen Forschungsstand zu Migrationsmotiven von Wissenschaftlern entwickelt (vgl. Abschnitt 4.3.5).

Idealtypisch wird dabei zwischen beruflichen, privaten und gesellschaftlichen Gründen unterschieden (vgl. Abbildung 6.43). Hinsichtlich der Argumente für die Zuwanderung nach Deutschland zeigt sich, dass berufliche Gründe wie die berufliche Perspektive, berufliche Kontakte und gute Forschungsbedingungen am häufigsten genannt werden. Die Befunde entsprechen weitgehend den Erkenntnissen des internationalen Forschungstandes zu Motiven der Migration bei Wissenschaftlern (vgl. Abschnitt 4.3.5). Für knapp ein Drittel der Befragten waren auch familiäre Gründe und Partnerschaft im Rahmen der Migrationsentscheidung von zentraler Bedeutung. Bei Wegner (2016b) verwiesen lediglich ca. $14 \%$ auf familiäre Gründe und Partnerschaft (vgl. Abschnitt 4.3.5). Diesbezüglich wurde geprüft, ob evtl. das Zuwanderungsalter die Unterschiede erklären kann. Allerdings zeigt sich, dass für Student Migrants (31\%) familiäre Motive sogar noch etwas häufiger eine Rolle gespielt haben als für Professional Migrants. Bei der Analyse der Zuwanderungsgründe ist zudem zu berücksichtigen, dass zum Zeitpunkt der Migrationsentscheidung häufig nur ein vorübergehender Aufenthalt geplant ist und der Entschluss, in Deutschland zu bleiben, erst im Laufe der Zeit gefasst wird. Dieses aus der Migrationsforschung bekannte Phänomen zeigt sich auch bei den Professoren mit Migrationshintergrund. Insgesamt $22 \%$ der Befragten hatten ursprünglich geplant, nur temporär in Deutschland zu leben, während für $39 \%$ die Entscheidung zu diesem Zeitpunkt noch offen war. Ebenfalls $39 \%$

\footnotetext{
${ }^{25}$ Dabei werden ausschließlich Student und Professional Migrants berücksichtigt, da davon auszugehen ist, dass bei den Early Migrants die Migrationsentscheidung in der Regel von den Eltern getroffen wurde.
} 
hatten indessen von Anfang an den Plan, dauerhaft in Deutschland zu bleiben. Das Phänomen, dass temporär geplante Aufenthalte in langfristige Migrationsprozesse münden, wird in ähnlicher Form in der Studie von Temme und Otto (2012) sichtbar (vgl. Abschnitt 4.3.6).

Ein Vergleich zwischen der Gruppe derer, die zunächst nur einen temporären Aufenthalt geplant hatten, und den Professoren, die von Anfang an dauerhaft in Deutschland bleiben wollten, zeigt, dass für die erste Gruppe generell deutlich weniger der genannten Gründe eine Rolle gespielt haben. Hinsichtlich der zweiten Gruppe zeigt sich, dass politische/religiöse Gründe, eine gute Gesundheitsversorgung, gute Bildungschancen für die Kinder oder familiäre Gründe/Partnerschaft sehr viel häufiger genannt werden.

Bei den Argumenten, die gegen eine Zuwanderung nach Deutschland sprachen, stehen insbesondere private und gesellschaftliche Gründe im Vordergrund. Am häufigsten wird auf die Trennung von Familie oder Partner hingewiesen, aber auch Bürokratie, die Umstellung auf andere Lebensbedingungen oder Sprachbarrieren stellten wichtige Gegenargumente dar (vgl. Abbildung 6.43).

In einem nächsten Schritt wird untersucht, inwieweit sich die Gründe bei der Zuwanderung nach soziodemographischen Merkmalen unterscheiden. Exemplarisch wurden die wichtigsten beruflichen, privaten und gesellschaftlichen Gründe näher beleuchtet.

Gute berufliche Perspektiven für die eigene Karriere werden als Argument von männlichen Professoren deutlich häufiger vorgebracht als von ihren Kolleginnen. Auch Professoren an Universitäten und Professional Migrants heben häufiger speziell diesen Grund hervor. Auffällig sind auch die Unterschiede zwischen den Generationen. Dabei hat die ältere Generation die Migrationsentscheidung am seltensten aufgrund der Karriereperspektive getroffen. Den größten Einfluss hatte die Karriereperspektive auf die Entscheidung der mittleren Generation, von der fast drei Viertel angeben, dass darin für sie ein wichtiges Motiv lag. Für die jüngere Generation spielt dieses Argument wiederum eine etwas geringere Rolle (vgl. Abbildung 6.44). Ein Grund hierfür könnte darin liegen, dass in dieser Generation über ein Drittel der Professoren eine Juniorprofessur innehat. Aufgrund der Befristung und der geringeren Bezahlung lässt sich vermuten, dass eine „karrieremotivierte“ Entscheidung bei Juniorprofessuren weniger ins Gewicht fällt. Das Argument des gehobenen Lebensstandards in Deutschland war bei den verschiedenen Gruppen von ähnlicher Relevanz.

Der wichtigste private Grund für die Zuwanderungsentscheidung sind familiäre Gründe/Partnerschaft. Besonders häufig verweisen Professoren aus den USA, Kanada und Australien, aus Fachhochschulen und aus der jüngeren Generation 


\section{Gründe für Zuwanderung}

Gute berufliche Perspektiven für die eigene Karriere Berufliche Kontakte

Gute Forschungsbedingungen

Familiäre Gründe/Partnerschaft

Guter Ruf einer bestimmten Hochschule

Lebensstandard in Deutschland

Guter Ruf der deutschen Hochschulen

Private Kontakte

Altersversorgung

Gute Gesundheitsversorgung

Gute Bildungschancen für meine Kinder

Politische/Religiöse Gründe

Keine Studiengebühren

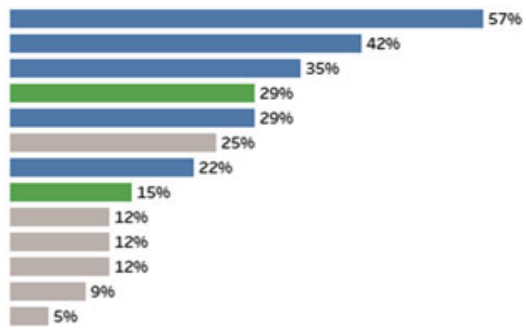

\section{Gründe gegen Zuwanderung}

Trennung von Familie/Partner/in

Bürokratie

Umstellung auf andere Lebensbedingungen

Sprachbarrieren

Aufgabe der bisherigen beruflichen Position

Auslănderfeindlichkeit

Dual-Career Problematik

Kinderbetreuung

Probleme bei der Anerkennung von Abschlüssen

Einwanderungsbestimmungen

Hohe Lebenshaltungskosten

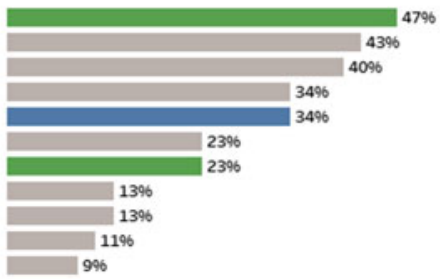

Berufliche Gründe

Private Gründe

Gesellschaftliche Gründe

Abbildung 6.43 Pro- und Kontra-Argumente hinsichtlich der Zuwanderung nach Deutschland. (Quelle: MOBIL 2012/2013, Gründe für Zuwanderung N = 109, Gründe gegen Zuwanderung $\mathrm{N}=49$ )

darauf, von denen jeweils fast die Hälfte dieses private Motiv hervorhebt (vgl. Abbildung 6.44).

Hinsichtlich der Argumente gegen eine Zuwanderung zeigen sich kaum Unterschiede zwischen den Gruppen bei wichtigen Motiven wie der Trennung von Familie oder Partner/in oder der Bürokratie. Interessante Unterschiede werden allerdings in Bezug auf das Gegenargument und zweithäufigste gesellschaftliche Motiv „Umstellung auf andere Lebensbedingungen“ sichtbar. Für deutlich über die Hälfte der Frauen, der Professoren aus nichtakademischen Elternhäusern und der Professoren aus Schwellen- und Entwicklungsländern war dieser Aspekt im Rahmen der Migrationsentscheidung von großer Bedeutung (vgl. Abbildung 6.45). Über die Ursachen dieser Unterschiede lassen sich lediglich Vermutungen aufstellen. Inwieweit diese Unterschiede möglicherweise auf 
Gute berufliche Perspektive für die Karriere

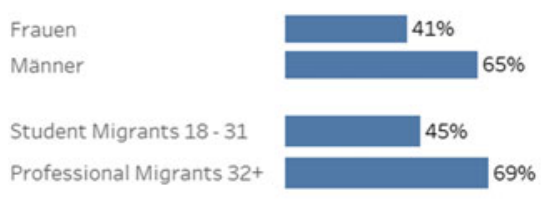

Jüngere Generation $<=44$

Mittlere Generation $45-53$

Altere Generation 54+

Fachhochschule

Universitat
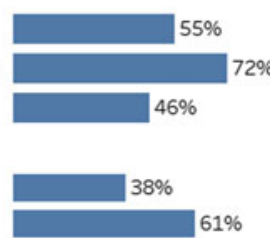

Familäre Gründe/Partnerschaft

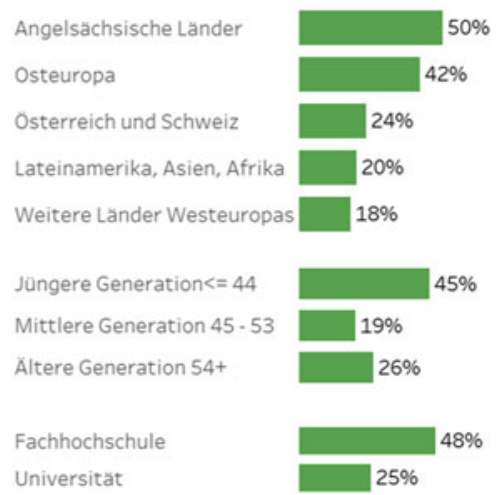

Abbildung 6.44 Soziodemographische Unterschiede hinsichtlich der wichtigsten beruflichen und privaten Gründe für die Zuwanderung nach Deutschland. (Quelle: MOBIL 2012/2013 Item 1: Geschlecht: $N=108$ ( $p=0,021)$, Migrationstypen: $N=109(p=0,015)$, Hochschulart: $\mathrm{N}=109$ ( $\mathrm{p}=0,053)$, Alter: $\mathrm{N}=108(\mathrm{p}=0,068)$; Item 2: Herkunftsregion: $\mathrm{N}$ $=109(\mathrm{p}=0,093)$ Hochschulart $\mathrm{N}=109(\mathrm{p}=0,041)$ Alter: $\mathrm{N}=108(\mathrm{p}=0,049) ; \mathrm{p}$-value basiert auf Chi-Quadrat-Test)

eine sozialisationsbedingt geringere Selbstwirksamkeitserwartung zurückzuführen sind oder ob den höheren Werten möglicherweise eine höhere interkulturelle Sensibilität zugrunde liegt, müsste in weiteren Studien untersucht werden.

Frauen
Männer
Eltern ohne Hochschulabschluss
Akademisches Elternhaus
Schwellen-/Entwicklungsland
Industrieland

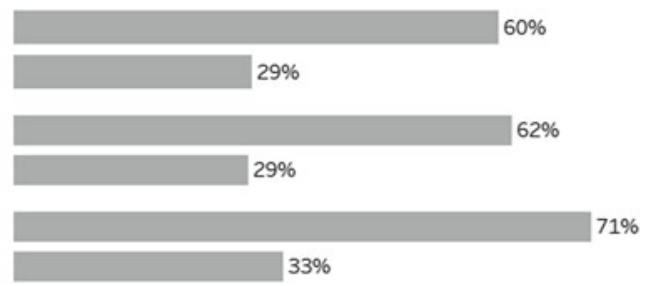

Abbildung 6.45 Umstellung auf andere Lebensbedingungen als Argument gegen eine Zuwanderung nach Deutschland. (Quelle: MOBIL 2012/2013 Geschlecht: N = 49 ( $\mathrm{p}=$ 0,043), Soziale Herkunft: $N=44(\mathrm{p}=0,043)$, Entwicklungsstatus, Herkunftsland: $\mathrm{N}=$ $49(\mathrm{p}=0,055)$; $\mathrm{p}$-value basiert auf Chi-Quadrat-Test) 


\subsubsection{Partnerschaft im Kontext der Migration und aktuell}

Wie die bisherigen Ausführungen zeigen, können private/familiäre Gründe einen großen Einfluss auf Migrationsentscheidungen bzw. -prozesse haben. Von zentraler Bedeutung - als mögliches Argument sowohl für als auch gegen die Migrationsentscheidung - ist dabei die Partnerschaft. Im Folgenden wird daher ausführlicher analysiert, in welchem Zusammenhang Partnerschaft und Migration unter Student und Professional Migrants stehen. Insgesamt befanden sich über die Hälfte der Professoren, die bei der Zuwanderung bereits volljährig waren, zu diesem Zeitpunkt in einer festen Partnerschaft. Student Migrants und Professorinnen waren dabei deutlich seltener in einer festen Partnerschaft als Professional Migrants und männliche Professoren. Etwa zwei Drittel haben in der Partnerschaft Unterstützung erhalten und sind gemeinsam mit dem Partner nach Deutschland migriert. Bei mehr als jedem siebtem Professor war die Migration allerdings auch mit Problemen in der Partnerschaft verbunden und bei jedem zehnten Professor führte sie sogar zur Trennung. Bei ca. jedem neunten Professor lebte der Partner bereits vorher in Deutschland (vgl. Abbildung 6.46).
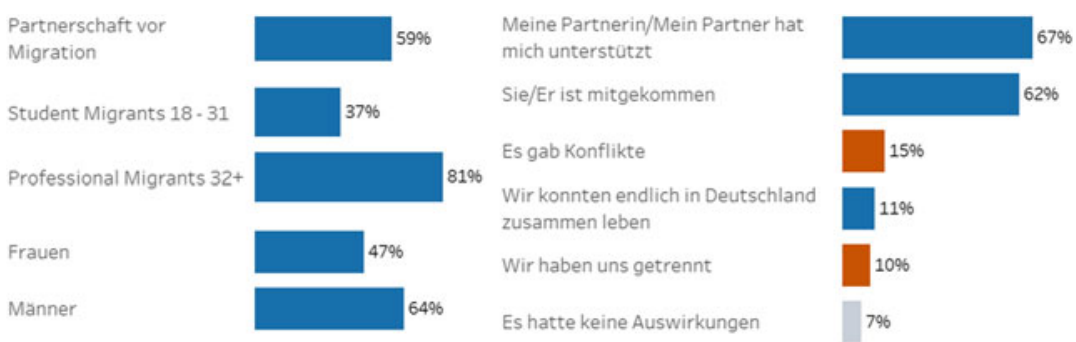

Abbildung 6.46 Partnerschaft vor der Zuwanderung und Auswirkungen der Migration auf die Partnerschaft. (Quelle: MOBIL 2012/2013, Partnerschaft vor Migration N = 109, Migrationstypen $\mathrm{N}=108(\mathrm{p}<0,001)$, Geschlecht $\mathrm{N}=107(\mathrm{p}=0,09)$, p-value basiert auf Chi-Quadrat-Test; Auswirkungen Migration auf Partnerschaft N = 61 [Mehrfachantworten])

Partner von männlichen Professoren sind häufiger mit nach Deutschland gekommen (67\%) als Partner von Professorinnen (47\%). Hier zeigen sich, wenngleich auch aufgrund der begrenzten Fallzahl unter Vorbehalt ${ }^{26}$, deutliche Anzeichen für eine Geschlechterdifferenz, und zwar dergestalt, dass Partner von

${ }^{26}$ Diese Ergebnisse sind statistisch nicht signifikant. 
Professorinnen sich deutlich seltener zu einer gemeinsamen Migration bereit erklären ${ }^{27}$.

Nach dem Mikrozensus 2017 leben $10 \%$ der Frauen mit einem Partner im selben Haushalt, der geringer qualifiziert ist, $63 \%$ der Paare haben Bildungsabschlüsse auf dem gleichen Niveau und in $27 \%$ der Fälle haben Männer einen höheren Bildungsabschluss als ihre Partnerin (vgl. Statistisches Bundesamt 2017a). Wenngleich der Bildungsstand des Partners nicht Teil der Erhebung war, lässt sich für Professoren mit Migrationshintergrund festhalten, dass ca. ein Fünftel der Professorinnen sich nicht in einer festen Partnerschaft befindet, während dies lediglich auf ein Zehntel der Professoren zutrifft.

Diese Differenz kann sowohl auf die genannten Geschlechterdifferenzen zurückzuführen sein, das heißt die Tatsache, dass hochqualifizierte Partnerinnen deutlich seltener geringer qualifizierte Partner haben, als auch auf die geringere Bereitschaft der Partner, gemeinsam das Land zu wechseln. Der deutlich geringere Anteil in fester Partnerschaft unter Professorinnen (80\%) im Vergleich zu ihren männlichen Kollegen (94\%) ist ebenfalls in der gesamten Professorenschaft in Deutschland zu beobachten (Jacob 2013; Teichler et al. 2017). Auffällig ist zudem der Zusammenhang zwischen Partnerschaft und Alter. Während deutlich über $90 \%$ der älteren Generation in einer festen Partnerschaft leben, trifft dies auf über ein Viertel der mittleren Generation nicht zu. In der jüngeren Generation liegt der Anteil der Professoren in einer festen Partnerschaft dann wieder deutlich über $90 \%$ (vgl. Abbildung 6.47).
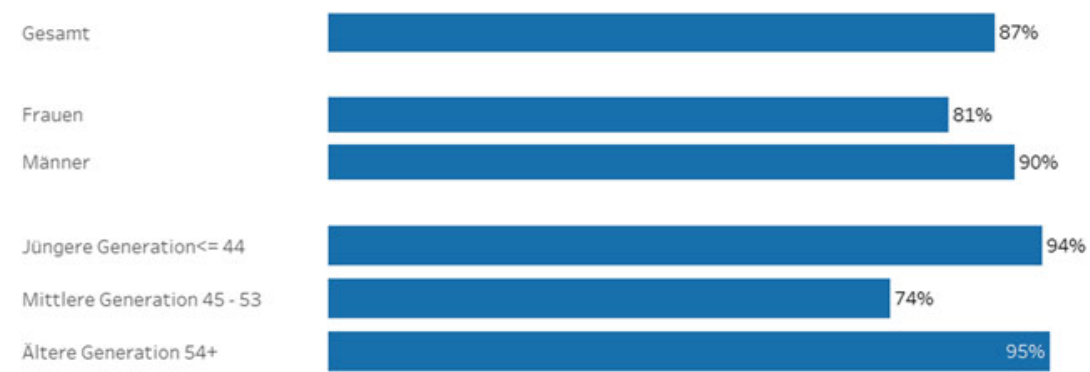

Abbildung 6.47 Feste Partnerschaft zum aktuellen Zeitpunkt nach Alter und Geschlecht. (Quelle: MOBIL 2012/2013, Gesamt $N=182$, Alter $N=180(p=0,001)$, Geschlecht $N=$ $180(\mathrm{p}=0,10)$, $\mathrm{p}$-value basiert auf Chi-Quadrat-Test $)$

${ }^{27}$ Dieser Befund gilt unabhängig von der möglichen Anzahl gleichgeschlechtlicher Partner. 
Im Kontext der Migration stellt sich zudem die Frage, in welchem Maße Professoren Partner aus Deutschland, aus dem Herkunftsland oder möglicherweise aus anderen Ländern haben. Über die Hälfte der Professoren mit Migrationshintergrund lebt in einer festen Partnerschaft mit einem Menschen aus Deutschland, während über ein Viertel einen Partner aus dem Herkunftsland und ca. jeder Sechste einen Partner aus einem anderen Land hat. Erwartungsgemäß haben Early Migrants am häufigsten Partner aus Deutschland, während die Mehrheit der Professional Migrants in einer Partnerschaft mit einem Menschen aus dem Herkunftsland lebt. Auffällig sind dabei auch die Unterschiede zwischen den Geschlechtern. So haben Professorinnen deutlich häufiger einen Partner aus Deutschland, während Professoren zu fast einem Drittel in einer Partnerschaft mit einem Menschen aus dem Herkunftsland leben (vgl. Abbildung 6.48). Wie sich in den folgenden Kapiteln noch zeigen wird (vgl. Abschnitt 6.7.4), hat die Wahl des Partners auch eine große Relevanz für die berufliche Zukunftsplanung.

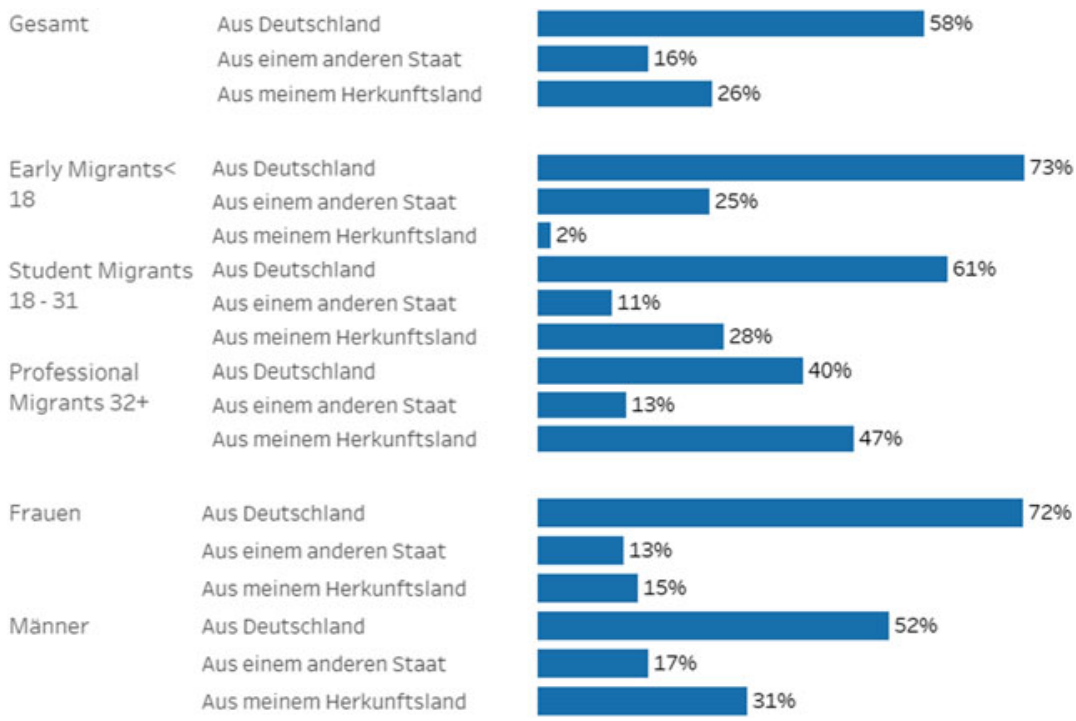

Abbildung 6.48 Herkunft des Partners nach Migrationstyp und Geschlecht. (Quelle: MOBIL 2012/2013, Gesamt $N=158$, Migrationstypen $N=158(\mathrm{p}<0,001)$, Geschlecht $\mathrm{N}=156(\mathrm{p}=0,05), \mathrm{p}$-value basiert auf Chi-Quadrat-Test $)$ 
Die Entscheidung des Partners für oder gegen eine gemeinsame Migration hängt in großem Maße von den Karrieremöglichkeiten am neuen Wohnort ab. Die Erkenntnis, dass es im Rahmen der Rekrutierung hochqualifizierten Personals von großer Bedeutung ist, auch Karriereperspektiven für den Partner zu schaffen, hat sich in den letzten Jahren auch zunehmend im Bereich der Personalrekrutierung durchgesetzt. Das klassische Rollenmodell mit erwerbstätigem Mann und haushaltsführender Frau wird zunehmend verdrängt durch ein Beziehungsmodell, in dem beide Partner berufstätig sind. ${ }^{28}$ Zunehmende Bedeutung erlangt in den letzten Jahren dabei das sogenannte dual-career model (Doppelkarrieremodell) (Abele 2010). Als dual-career couples werden dabei Partnerschaften bezeichnet, in denen beide Partner ,hochqualifiziert sind, eine lebenslange Karriereorientierung haben, ein hohes $\mathrm{Maß}$ an beruflicher Bindung aufweisen und ihren Lebensweg gemeinsam gehen wollen" (Abele 2010, S. 22).

Im Folgenden soll untersucht werden, inwieweit Annahmen des Doppelkarrieremodells auf Professoren mit Migrationshintergrund anwendbar sind. Dabei fällt zunächst auf, dass fast die Hälfte der Partner von Professoren mit Migrationshintergrund ebenfalls in der Wissenschaft tätig ist. Bei den Professional Migrants, also der Gruppe, die erst nach dem 32. Lebensjahr nach Deutschland migriert ist und zuvor fast durchgehend in einer Partnerschaft gelebt hat (vgl. Abbildung 6.46), arbeitet sogar deutlich über die Hälfte der Partner in der Wissenschaft. Zugleich zeigt sich, dass insbesondere Partner, die nicht aus Deutschland kommen, häufig in der Wissenschaft tätig sind. Zudem arbeiten auch Partner von Universitätsprofessoren deutlich häufiger in der Wissenschaft als Partner von Fachhochschulprofessoren (vgl. Abbildung 6.49).

Die Befunde geben klare Hinweise dafür, dass die Förderung des Doppelkarrieremodells bzw. eine zunehmende Unterstützung berufstätiger Partner eine besondere Relevanz für Universitäten bei der Anwerbung internationaler Professoren aus dem Ausland haben sollte. Es sollte sich aber nicht darauf beschränken. In Anbetracht der Zielsetzung, bestehende Genderdisparitäten bei der Vereinbarkeit von wissenschaftlicher Karriere und Familie zu überwinden, ist es von enormer Bedeutung, die Förderung von dual-career couples institutionell im deutschen Hochschulwesen zu verankern, da vor allem Partner von Professorinnen, also größtenteils Männer, nur selten bereit sind, die eigene Karriere zugunsten des Partners zurückzustellen.

Bei über der Hälfte der Professoren hat der Partner einen Arbeitsplatz in derselben Region gefunden. Bei jedem zehnten Professor wohnt der Partner hingegen

\footnotetext{
${ }^{28}$ Wenngleich Frauen deutlich häufiger in Teilzeit beschäftigt sind.
} 


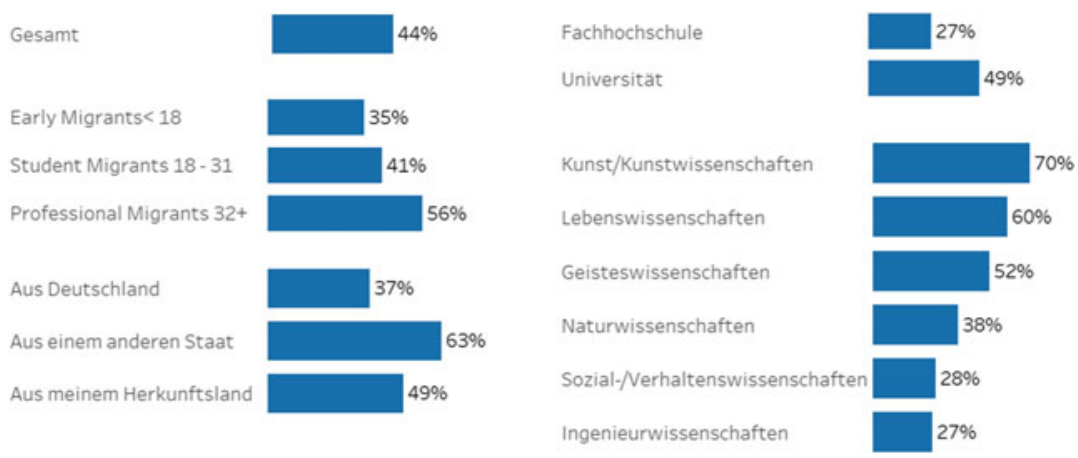

Abbildung 6.49 Berufliche Tätigkeit des Partners in der Wissenschaft nach Migrationstyp, Herkunft des Partners, Hochschulart und Fächergruppe. (Quelle: MOBIL 2012/2013, Gesamt $\mathrm{N}=152$, Migrationstypen $\mathrm{N}=152(\mathrm{p}=0,09)$, Herkunft Partner/in $\mathrm{N}=152(\mathrm{p}=0,06)$, Hochschulart $\mathrm{N}=152(\mathrm{p}<0,03)$, Fächergruppen $\mathrm{N}=146(\mathrm{p}=0,02) \mathrm{p}$-value basiert auf Chi-Quadrat-Test)

in einem anderen Staat und bei $15 \%$ der Befragten ist der Partner nicht erwerbstätig (vgl. Abbildung 6.50). Dabei stellt sich die Frage, inwieweit Erwerbslosigkeit oder „Fernbeziehungen“ möglicherweise auch bewusste Entscheidungen zugrunde liegen oder ob es in Bezug auf den Partner primär an adäquaten Beschäftigungsmöglichkeiten vor Ort fehlt. Daher wurde spezifisch nach dem Erfolg im Rahmen der Arbeitsplatzsuche des Partners gefragt.
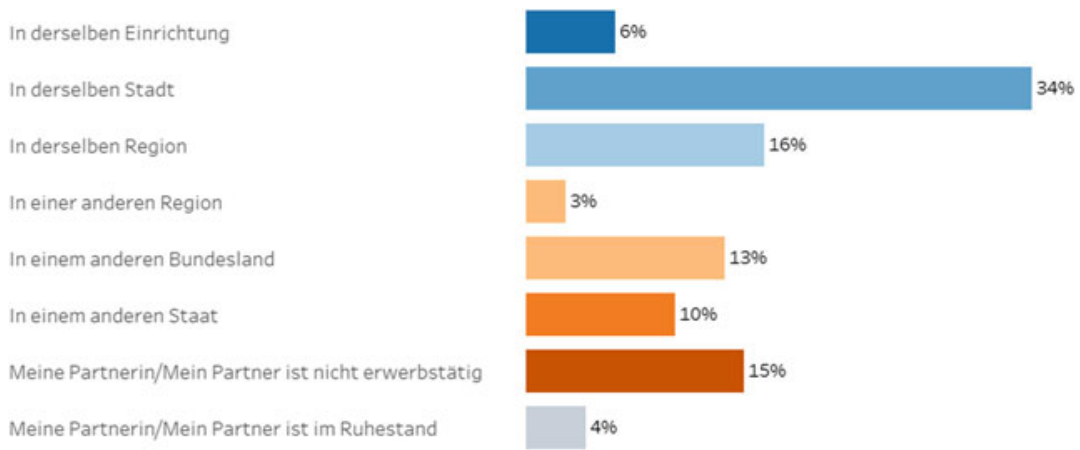

Abbildung 6.50 Arbeitsort Partner. (Quelle: MOBIL 2012/2013, N = 151) 
Zunächst wird differenziert, inwieweit eine Arbeitsplatzsuche des Partners vor Ort überhaupt stattgefunden hat. Bei ca. einem Drittel ist es aus zwei Gründen zu keiner Arbeitsplatzsuche gekommen. Zum einen kann es sein, dass der Partner bereits vor der Migration einen Arbeitsplatz in derselben Region hatte und der Entscheidung, eine Professur in Berlin oder Hessen zu übernehmen, möglicherweise auch die Motivation zugrunde lag, zum Partner zu ziehen. Zum anderen besteht die Möglichkeit, dass der Partner nicht bereit war, gemeinsam zu migrieren, sei es aus familiären, beruflichen oder sonstigen Gründen. Interessante Unterschiede zeigen sich wiederum zwischen den Geschlechtern. Bei lediglich $16 \%$ der männlichen Professoren hatte der Partner zum Zeitpunkt der Migration bereits eine Tätigkeit vor Ort, während ca. ein Drittel der Professorinnen die Migrationsentscheidung möglicherweise auch getroffen hat, um in der Nähe des Partners leben zu können (vgl. Abbildung 6.51).
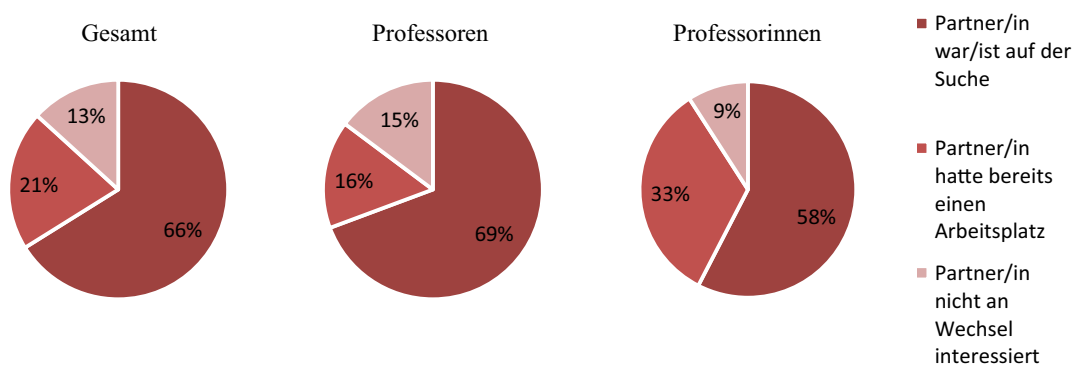

Abbildung 6.51 Unterschiede Beschäftigungssituation des Partners nach Geschlecht. (Quelle: MOBIL 2012/2013, N = 121 ( $\mathrm{p}=0,10$ ), p-value basiert auf Chi-Quadrat-Test)

Für die Partner, die sich auf Arbeitsplatzsuche begeben haben, hat sich diese insgesamt schwierig gestaltet. Über $40 \%$ der Partner haben bisher keinen Arbeitsplatz gefunden. Jeder fünfte Partner hat die Suche nach einer Stelle bereits aufgegeben. Diese Zahlen verdeutlichen den hohen Handlungsbedarf bezüglich dual-career programmes für Partner berufstätiger Migranten. Sowohl Qualifikation und Berufsfeld des Partners als auch die Frage der adäquaten Beschäftigung für die Partner, die einen Arbeitsplatz gefunden haben, sollten in Folgestudien noch expliziter untersucht werden. Insgesamt zeigt sich, dass fast zwei Drittel der Partner von männlichen Professoren einen Arbeitsplatz gefunden haben, während dies nur knapp über $40 \%$ der Partner von Professorinnen gelungen ist (vgl. Abbildung 6.52). Zudem gestaltet sich die Arbeitsplatzsuche für Partner, die 
ebenfalls in der Wissenschaft tätig sind, besonders schwierig. Fast zwei Drittel (65\%) haben keinen Arbeitsplatz in der Nähe gefunden. Unter Berücksichtigung der Tatsache, dass dual-career programmes an Hochschulen sich insbesondere an Wissenschaftlerpaare richten, sollten auch diese Zahlen im Rahmen zukünftiger hochschulpolitischer Maßnahmen in besonderem Maße berücksichtigt werden.

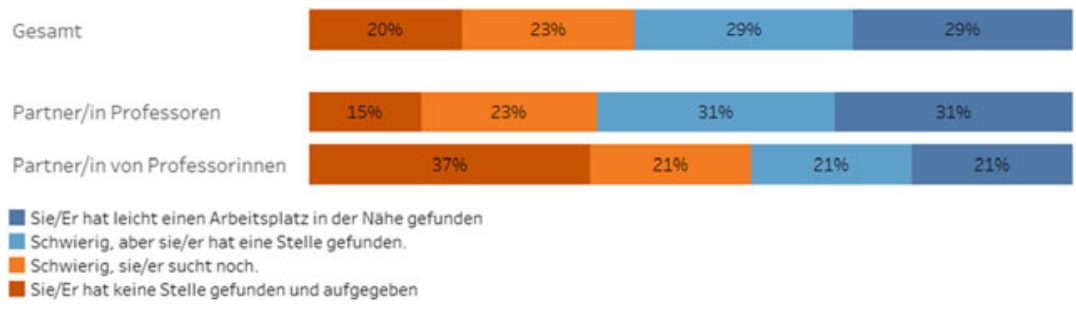

Abbildung 6.52 Erfolg bei der Arbeitsplatzsuche des Partners nach Geschlecht. (Quelle: MOBIL 2012/2013, $\mathrm{N}=80$ ( $\mathrm{p}=0,083)$, $\mathrm{p}$-value basiert auf Mann-Whitney-U-Test)

\subsubsection{Vereinbarkeit von Elternschaft und Karriere}

Im Rahmen der Forschung über wissenschaftliche Karrieren liegt ein besonderes Augenmerk auf der Frage der Vereinbarkeit von Familie und Beruf. Dabei spielt insbesondere die Frage eine zentrale Rolle, inwieweit sich eine erfolgreiche wissenschaftliche Karriere und Elternschaft vereinbaren lassen. Studien belegen, dass sich die Vereinbarkeit in besonderem Maße für Frauen schwierig gestaltet. Das Problem der Vereinbarkeit lässt sich als wichtige Ursache für das bestehende Ungleichverhältnis der Geschlechter im Professorenberuf einordnen. So müssen sich Frauen häufiger zwischen beruflicher Karriere und Elternschaft entscheiden (vgl. Lind und Samskje 2010).

Vor diesem Hintergrund wird im Folgenden für die Professoren mit Migrationshintergrund systematisch untersucht, inwieweit sich strukturelle Unterschiede bei der Elternschaft zwischen männlichen Professoren und Professorinnen zeigen. Zudem stellt sich die Frage, welche Rolle der Migrationsprozess in diesem Zusammenhang spielt. Es ist anzunehmen, dass Elternschaft und Karriere sich für Professoren, die schon in Deutschland geboren und/oder aufgewachsen sind, leichter vereinbaren lassen als für Professoren, die erst im Laufe ihres Studiums 
oder während der Berufstätigkeit nach Deutschland gekommen sind, da diese häufig erst ein neues soziales Umfeld aufbauen müssen und zugleich häufiger Partner haben, die nicht in derselben Region leben.

Zunächst zeigt sich, dass über zwei Drittel der Professoren mit Migrationshintergrund Kinder haben. Bei männlichen Professoren liegt der Anteil sogar bei fast drei Vierteln, während lediglich etwas über die Hälfte der Professorinnen Kinder hat (vgl. Abbildung 6.53). Die Geschlechterdifferenz lässt sich in ähnlicher Form bei der gesamten Professorenschaft in Deutschland beobachten. Hier leben $67 \%$ der männlichen Universitätsprofessoren, aber nur $33 \%$ der Professorinnen mit Kindern in einem Haushalt (Jacob 2013; vgl. auch Teichler et al. 2017).

Hinsichtlich des Zuwanderungszeitpunkts fällt auf, dass Early Migrants mit Abstand am seltensten (56 \%) und Student Migrants mit Abstand am häufigsten Kinder haben (82\%). Dabei unterscheidet sich der Frauenanteil zwischen den beiden Gruppen nicht. Ursachen für diese Unterschiede lassen sich an dieser Stelle nicht klar benennen. Folgestudien sollten der Frage nachgehen, ob Early Migrants sich häufiger bewusst gegen Kinder entscheiden oder inwieweit möglicherweise spezifische Probleme der Vereinbarkeit von Elternschaft und Karriere für diese Gruppe bestehen.

Die durchschnittliche Kinderzahl liegt für alle Professoren mit Migrationshintergrund bei 1,37, für männliche Professoren bei 1,56 und für Professorinnen lediglich bei 0,98 (vgl. Abbildung 6.53). Professoren mit Kindern haben zumeist ein Kind (29\%) oder zwei Kinder (48 \%), während lediglich knapp ein Viertel $(23 \%)$ mehr als zwei Kinder vorweisen kann. Die besonders im Rahmen populistischer Debatten häufig vorgebrachte These, dass alle Migranten überdurchschnittlich viele Kinder zur Welt bringen, lässt sich somit für die Gruppe der Professoren mit Migrationshintergrund definitiv nicht bestätigen.

Aus hochschulpolitischer Sicht ist insbesondere die Frage interessant, inwieweit die Professoren minderjährige Kinder haben, da diese Gruppe hinsichtlich der Vereinbarkeit von Elternschaft und Karriere von zentraler Bedeutung ist. Analysen bezüglich des Alters des jüngsten Kindes zeigen, dass über ein Viertel der Professoren Kinder hat, die sich im Elementarbereich befinden, sodass diese Gruppe in Bezug auf Betreuungsangebote durch Kindertagesstätten berücksichtigt werden sollte (vgl. Abbildung 6.53). Zugleich hat über ein Fünftel der Professoren Kinder im Grundschulalter (Primarbereich). Auch hier stellt sich die Frage, inwieweit bspw. in Form von Ganztagsschulen Möglichkeiten bestehen, die Vereinbarkeit von Elternschaft und Beruf zu erleichtern. In Studien über die Vereinbarkeit von Kind und Berufstätigkeit wird zum Teil vorgebracht, dass sich die Frage der Vereinbarkeit gerade bei Professoren möglicherweise überhaupt nicht stelle, wenn das Kind nicht im eigenen Haushalt lebt. Unabhängig davon, dass 
auch Eltern, die nicht im selben Haushalt wie ihre Kinder leben, in hohem Maße Verantwortung im Rahmen der Kindererziehung übernehmen können, zeigt sich empirisch, dass deutlich über $90 \%$ der minderjährigen Kinder von Professoren mit Migrationshintergrund auch im selben Haushalt wie der befragte Elternteil leben.

In den letzten Jahrzehnten hat sich in Deutschland das Durchschnittsalter bei der Geburt des ersten Kindes kontinuierlich erhöht. Dieser Trend gilt in besonderem Maße für Akademiker, da diese eine Elternschaft häufig erst dann in Erwägung ziehen, wenn sie ein abgeschlossenes Studium, ein festes Einkommen und ein unbefristetes Beschäftigungsverhältnis vorweisen können. Dementsprechend gestaltet sich das Thema Elternschaft im Rahmen wissenschaftlicher Karrieren oftmals schwierig, da unbefristete Beschäftigungsverhältnisse in diesem Bereich nur selten bzw. erst spät erreicht werden können, nämlich primär durch Berufung auf eine Professur mit durchschnittlich ca. 40 Jahren.

Auch bei Professoren mit Migrationshintergrund zeigt sich ein vergleichsweises hohes Durchschnittsalter bei der Geburt des ersten Kindes (31,8 Jahre). Dabei liegt das Alter der Professorinnen (31,0) etwas unter dem Alter der männlichen Professoren (32,1 Jahre) (vgl. Abbildung 6.53). Die Unterschiede erklären sich primär darüber, dass jeder sechste männliche Professor erst mit über 37 Jahren das erste Mal Vater geworden ist, während Professorinnen bei der Geburt ihres jüngsten Kindes maximal 37 Jahre alt waren. Die Tatsache, dass sich die Vereinbarkeit von Kind und Karriere in besonderem Maße für Frauen als problematisch erweist, wird häufig auf spezifische Formen der Arbeitsteilung zwischen Männern und Frauen zurückgeführt. Das heißt, dass die geringere Bereitschaft von Männern, sich gleichberechtigt an der Kindererziehung zu beteiligen, die Ungleichheiten zwischen den Geschlechtern verstärkt. Es ließe sich allerdings auch die Frage stellen, inwieweit der Umstand, dass für Männer eine Elternschaft auch noch in deutlich höherem Alter biologisch möglich ist, die Unterschiede erklären kann. ${ }^{29}$ Um dieser Frage nachzugehen, wurden die Geschlechterunterschiede ausschließlich für Eltern untersucht, die bei der Geburt des ersten Kindes maximal 37 Jahre alt waren. Der Anteil der männlichen Professoren mit Kindern beläuft sich dann nur noch auf $67 \%$, liegt damit aber weiterhin statistisch signifikant über dem Anteil der Professorinnen mit Kindern (53\%). Die Ergebnisse verdeutlichen, wie wichtig es ist, weiterhin auf gesellschaftlicher Ebene für eine stärkere

\footnotetext{
${ }^{29}$ Selbst wenn die Unterschiede ausschließlich darauf zurückzuführen wären, müsste gesellschaftlich die Frage gestellt werden, welche spezifischen Möglichkeiten Frauen eröffnet werden sollten, um eine Vereinbarkeit in dem „kleineren Zeitfenster“ zu ermöglichen.
} 
Gleichberechtigung der Geschlechter hinsichtlich der Frage der Vereinbarkeit von Elternschaft und Berufstätigkeit zu sorgen.

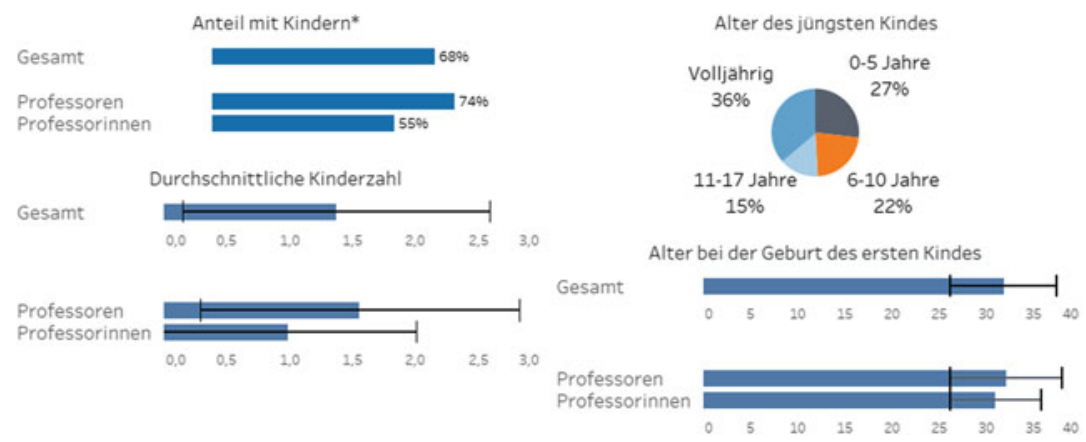

Abbildung 6.53 Geschlechterunterschiede bei der Elternschaft. (Quelle: MOBIL 2012/2013, Anteil mit Kindern $N=176$, Geschlechterunterschiede $N=175,(p=0,015)$ p-value basiert auf Chi-Quadrat-Test; Durchschnittliche Kinderzahl N = 174, Geschlechterunterschiede $\mathrm{N}=173(\mathrm{p}=0,003) \mathrm{p}$-value basiert auf einfaktorieller ANOVA. Alter jüngstes Kind $\mathrm{N}=108$. Alter bei Geburt erstes Kind $\mathrm{N}=108$, Geschlechterunterschiede $\mathrm{N}=107$ (p $=0,35)$, p-value basiert auf einfaktorieller ANOVA; *Bei sogenannten Patchwork-Familien sind alle Kinder gemeint, auch die Kinder des Partners; $\longmapsto$ Standardabweichung)

\subsubsection{Berufliche Zukunftsplanung}

Im Folgenden soll näher untersucht werden, inwieweit Professoren mit Migrationshintergrund im Kontext von Zuwanderung ihre berufliche Zukunft in Deutschland planen. Wie es gelingen kann, hochqualifiziertes Personal aus dem Inund Ausland in Deutschland zu halten, stellt in diesem Zusammenhang eine der Schlüsselfragen im internationalen Wettbewerb um hochqualifizierte Spitzenkräfte dar (vgl. Abschnitt 3.2.3). Im Folgenden werden Analysen vorgenommen, um herauszufinden, welche Einflussfaktoren für eine berufliche Zukunftsplanung in Deutschland eine wichtige Rolle spielen. Insgesamt planen fast zwei Drittel der Professoren (64\%) ihre langfristige berufliche Zukunft in Deutschland. Gleichzeitig können sich $26 \%$ der Professoren ihre berufliche Zukunft sowohl in Deutschland als auch im Ausland vorstellen. Jeder zehnte Professor mit Migrationshintergrund plant seine berufliche Zukunft im Ausland. 
Im Folgenden wird zunächst bivariat untersucht, welchen Einfluss soziodemographische Merkmale, die berufliche Zufriedenheit, das Herkunftsland des Partners, Diskriminierungserfahrungen, private soziale Kontakte und internationale berufliche Aktivitäten auf die Bleibeabsicht haben. Auf dieser Grundlage soll in einem zweiten Schritt über das Verfahren der logistischen Regression der multivariate Einfluss der wichtigsten Variablen untersucht werden. Um eine valide Samplegröße insbesondere für das multivariate Verfahren zu erreichen, wird die abhängige Variable binär codiert in Professoren, die ihre Zukunft eindeutig in Deutschland planen (64\%), und Professoren, deren berufliche Zukunftsplanung noch offen ist oder die planen, ins Ausland zu wechseln (36\%).

Ein auffälliger Zusammenhang zeigt sich zwischen der Aufenthaltsdauer in Deutschland und der beruflichen Zukunftsplanung. Über die Hälfte der Professoren, die erst in den letzten zehn Jahren zugewandert sind, plant ihre Zukunft nicht eindeutig in Deutschland, während Professoren, deren Zuwanderung bereits mehr als zehn Jahre zurückliegt, zu über $70 \%$ ihre berufliche Zukunft eindeutig in Deutschland planen. Dieser Befund lässt sich vermutlich primär darauf zurückführen, dass Kontakte und Kommunikation zum Herkunftsland oder in andere Staaten noch stärker ausgeprägt sind und somit eine erneute Migration leichter möglich ist. Zugleich sind Netzwerkstrukturen und die kulturelle und soziale Teilhabe bei Professoren, die bereits länger in Deutschland leben, stärker ausgeprägt (vgl. Abschnitt 6.8.1), was möglicherweise auch die Bleibeabsicht in Deutschland stärkt. Überraschend ist allerdings, dass fast die Hälfte der Professoren,

Tabelle 6.13 Unterschiede hinsichtlich der Bleibeabsicht nach soziodemographischen Merkmalen

\begin{tabular}{|c|c|c|c|c|}
\hline Kategorien & Variable & $\begin{array}{l}\text { Berufl. Zukunft } \\
\text { Ausland/offen }\end{array}$ & $\begin{array}{l}\text { Sig. } \\
\left(\chi^{2}-\text { Test }\right)\end{array}$ & $\mathrm{N}$ \\
\hline \multirow{9}{*}{$\begin{array}{l}\text { Merkmale } \\
\text { zur Migration }\end{array}$} & Gesamt & $36 \%$ & & 174 \\
\hline & Early Migrants $<18$ & $40 \%$ & \multirow[t]{3}{*}{0,12} & \multirow[t]{3}{*}{174} \\
\hline & Student Migrants 18-31 & $26 \%$ & & \\
\hline & Professional Migrants $32+$ & $43 \%$ & & \\
\hline & Max. 10 Jahre in Deutschland & $54 \%$ & \multirow[t]{4}{*}{$0,04 *$} & \multirow[t]{4}{*}{172} \\
\hline & Zwischen 11-20 Jahren & $22 \%$ & & \\
\hline & Über 20 Jahre in Deutschland & $26 \%$ & & \\
\hline & Zweite Generation & $47 \%$ & & \\
\hline & Deutsch mit $\mathrm{MgH}$ & $30 \%$ & $0,06 *$ & 174 \\
\hline
\end{tabular}


Tabelle 6.13 (Fortsetzung)

\begin{tabular}{|c|c|c|c|c|}
\hline Kategorien & Variable & $\begin{array}{l}\text { Berufl. Zukunft } \\
\text { Ausland/offen }\end{array}$ & $\begin{array}{l}\text { Sig. } \\
\left(\chi^{2}-\text { Test }\right)\end{array}$ & $\mathrm{N}$ \\
\hline & Ausländische StA. & $43 \%$ & & \\
\hline & Österreich und Schweiz & $43 \%$ & \multirow[t]{5}{*}{0,56} & \multirow[t]{5}{*}{173} \\
\hline & Weitere Länder Westeuropas & $38 \%$ & & \\
\hline & Osteuropa & $23 \%$ & & \\
\hline & Lateinamerika, Asien und Afrika & $33 \%$ & & \\
\hline & Angelsächsiche Länder ${ }^{\mathrm{a}}$ & $37 \%$ & & \\
\hline & Industrieland & $39 \%$ & \multirow[t]{2}{*}{$0,04 *$} & \multirow[t]{2}{*}{173} \\
\hline & Schwellen-/Entwicklungsland & $17 \%$ & & \\
\hline \multirow{7}{*}{$\begin{array}{l}\text { Demographische } \\
\text { Merkmale }\end{array}$} & Jüngere Generation $<=44$ & $49 \%$ & \multirow[t]{3}{*}{$0,02 *$} & \multirow[t]{3}{*}{173} \\
\hline & Mittlere Generation 45-53 & $31 \%$ & & \\
\hline & Ältere Generation 54+ & $24 \%$ & & \\
\hline & Männer & $39 \%$ & \multirow[t]{2}{*}{0,20} & \multirow[t]{2}{*}{173} \\
\hline & Frauen & $29 \%$ & & \\
\hline & First Generation & $32 \%$ & \multirow[t]{2}{*}{0,47} & \multirow[t]{2}{*}{153} \\
\hline & $\begin{array}{l}\text { Min. ein Elternteil } \\
\text { Hochschulabschluss }\end{array}$ & $38 \%$ & & \\
\hline \multirow{12}{*}{$\begin{array}{l}\text { Berufliche } \\
\text { Merkmale }\end{array}$} & Juniorprofessur (Uni) & $63 \%$ & \multirow[t]{4}{*}{$0,01 * *$} & \multirow[t]{4}{*}{174} \\
\hline & Fachhochschulprofessur & $20 \%$ & & \\
\hline & W2/C3/C2-Professur (Uni) & $37 \%$ & & \\
\hline & W3/C4-Professur (Uni) & $34 \%$ & & \\
\hline & Geisteswissenschaften & $45 \%$ & \multirow[t]{6}{*}{0,43} & \multirow[t]{6}{*}{167} \\
\hline & Sozial-/Verhaltenswissenschaften & $44 \%$ & & \\
\hline & Kunst/Kunstwissenschaften & $36 \%$ & & \\
\hline & Lebenswissenschaften & $21 \%$ & & \\
\hline & Naturwissenschaften & $33 \%$ & & \\
\hline & Ingenieurwissenschaften & $25 \%$ & & \\
\hline & Humanities & $42 \%$ & \multirow[t]{2}{*}{$0,07 *$} & \multirow[t]{2}{*}{167} \\
\hline & Science & $29 \%$ & & \\
\hline
\end{tabular}

Quelle: MOBIL 2012/2013; a (USA, Kanada, Australien); Signifikanzlevel: $* p \leq 0,1$, $* * \mathrm{p} \leq 0,01$ 
die bereits in zweiter Generation in Deutschland leben, die Zukunft nicht eindeutig in Deutschland plant (vgl. Tabelle 6.13). Über die Gründe ließe sich an dieser Stelle lediglich spekulieren. Dennoch sollte dieser Befund als wichtiger Hinweis verstanden werden, dass zukünftige Forschungsprojekte und politische Maßnahmen auch dieser Gruppe verstärkt Beachtung schenken und nicht nur zugewanderte Spitzenkräfte in den Blick nehmen sollten. Ein wichtiger Faktor in diesem Zusammenhang stellt das Alter dar. Während fast die Hälfte der jüngeren Generation in Erwägung zieht, in Zukunft ins Ausland zu wechseln, trifft dies in der mittleren Generation auf nur knapp ein Drittel und in der älteren Generation auf weniger als ein Viertel zu. Ein Grund könnte in der spezifischen Lebensphase liegen. Aus der Migrationsforschung ist bekannt, dass insbesondere junge Erwachsene in hohem Maße migrieren. Ab Mitte 30 besteht ein weitgehend negativer Zusammenhang zwischen Alter und Migrationsanteil, abgesehen von einem kurzen Anstieg nach dem Ruhestand, da zu dem Zeitpunkt nochmal eine größere Gruppe in das Herkunftsland zurückkehrt. Ein Grund könnte in den stärker konsolidierten Lebensläufen von älteren Menschen liegen, während bei jüngeren Menschen möglicherweise Neugier und Karriereorientierung eine größere Rolle spielen (Zaiceva, 2014).

Ein weiterer Grund für den hohen Anteil in der jüngeren Generation könnte zudem darin liegen, dass hier fast $40 \%$ der Befragten als Juniorprofessoren beschäftigt sind. Unter Juniorprofessoren sind es fast zwei Drittel der Befragten, die einen Wechsel ins Ausland erwägen, was insbesondere mit dem zumeist befristeten Beschäftigungsverhältnis sowie der deutlich geringeren Bezahlung zusammenhängen dürfte. Zugleich kann es sein, dass die Gruppe aufgrund der noch ausstehenden langen Berufstätigkeit sich häufiger Zukunftsperspektiven sowohl in Deutschland als auch im Ausland offenhält. Weitere auffällige Unterschiede zeigen sich nach Entwicklungsstand des Herkunftslandes und nach Fächergruppen. Über 80 \% der Professoren aus Entwicklungs- und Schwellenländern planen definitiv, in Deutschland zu bleiben. Professoren aus dem Bereich Humanities (Sozial-/Verhaltenswissenschaften, Geisteswissenschaften, Kunst/Kunstwissenschaften) ziehen hingegen etwas häufiger einen Wechsel ins Ausland in Betracht, als Professoren im Bereich Science (vgl. Tabelle 6.13).

Von zentraler Bedeutung für die Bleibeabsicht ist die berufliche Zufriedenheit. Über $70 \%$ der unzufriedenen Professoren ziehen in Erwägung, ins Ausland zu wechseln. Der entsprechende Anteil liegt bei eher zufriedenen Professoren bei weniger als einem Drittel, bei voll und ganz zufriedenen Professoren sogar bei weniger als einem Fünftel (vgl. Tabelle 6.14) 
Tabelle 6.14 Unterschiede hinsichtlich der Bleibeabsicht nach beruflicher Zufriedenheit, Herkunftsland Partner/in und Diskriminierungserfahrung

\begin{tabular}{|c|c|c|c|c|}
\hline Kategorien & Variable & $\begin{array}{l}\text { Berufl. Zukunft } \\
\text { Ausland/offen }\end{array}$ & Sig. ( $x$ 2-Test) & $\mathrm{N}$ \\
\hline \multirow[t]{5}{*}{ Zufriedenheit } & Gesamt & $36 \%$ & & 174 \\
\hline & $\begin{array}{l}\text { Stimme überhaupt } \\
\text { oder eher nicht zu }\end{array}$ & $71 \%$ & \multirow[t]{4}{*}{$<0,001 * * *$} & \multirow[t]{4}{*}{173} \\
\hline & Teils teils & $57 \%$ & & \\
\hline & Stimme eher zu & $30 \%$ & & \\
\hline & $\begin{array}{l}\text { Simme voll und ganz } \\
\text { zu }\end{array}$ & $18 \%$ & & \\
\hline \multirow{4}{*}{$\begin{array}{l}\text { Herkunftsland } \\
\text { Partner/in }\end{array}$} & Deutschland & $25 \%$ & \multirow[t]{4}{*}{$0,002 * *$} & \multirow[t]{4}{*}{168} \\
\hline & Herkunftsland & $44 \%$ & & \\
\hline & Anderer Staat & $67 \%$ & & \\
\hline & Keine Partnerschaft & $36 \%$ & & \\
\hline \multirow{2}{*}{$\begin{array}{l}\text { Diskriminierung } \\
\text { aufgrund von } \\
\text { Herkunft oder } \\
\text { Religion }\end{array}$} & Nachteile & $41 \%$ & \multirow[t]{2}{*}{0,42} & \multirow[t]{2}{*}{157} \\
\hline & Keine Nachteile & $34 \%$ & & \\
\hline
\end{tabular}

Quelle: MOBIL 2012/2013; Signifikanzlevel: *p $\leq 0,1, * * \mathrm{p} \leq 0,01, * * * \mathrm{p} \leq 0,001$

Große Relevanz hat in diesem Zusammenhang auch das Herkunftsland des Partners bzw (Tabelle 6.14). der Partnerin. Die Möglichkeit, ins Ausland zu wechseln, wird deutlich häufiger in Betracht gezogen, wenn der Partner aus dem Herkunftsland oder aus einem anderen Staat kommt. Ein wichtiger Faktor ist zudem das Herkunftsland des Partners (vgl. Tabelle 6.13). Die starke Bleibeabsicht in Zusammenhang mit einer Partnerschaft mit einer deutschen Person zeigt sich in ähnlicher Form bei der Studie von Wegner (2016b) über internationale Nachwuchswissenschaftler (vgl. Abschnitt 4.3.6).

Auch zwischen der Bleibeabsicht und der Pflege privater sozialer Kontakte besteht ein Zusammenhang. Professoren, die sehr häufig privaten Kontakt zu Deutschen haben, planen auch deutlich häufiger ihre berufliche Zukunft in Deutschland. So scheint der regelmäßige soziale Kontakt zu Deutschen die Professoren mit Migrationshintergrund oftmals darin $\mathrm{zu}$ bestärken, ihre berufliche Zukunft in Deutschland zu verbringen. Private Teilhabe sollte dementsprechend auch als ein möglicher wichtiger Faktor berücksichtigt werden. Zugleich zeigt 
sich, dass Professoren, die regelmäßigen Kontakt zu Menschen aus anderen Ländern haben, deutlich häufiger einen Wechsel ins Ausland in Betracht ziehen (vgl. Tabelle 6.15). Da der Übergang zwischen beruflichen und privaten Kontakten oftmals fließend verläuft (vgl. Abschnitt 4.3.7), können diese Kontakte ins Ausland durchaus auch Netzwerkstrukturen darstellen, die die Voraussetzungen für einen Wechsel ins Ausland erst schaffen oder zumindest begünstigen.

Tabelle 6.15 Unterschiede hinsichtlich der Bleibeabsicht nach privater, sozialer Interaktion mit verschiedenen Gruppen

\begin{tabular}{l|l|l|l|l|l}
\hline $\begin{array}{l}\text { Index soziale } \\
\text { Interaktion von nie } \\
\text { (1) bis oft (3) }\end{array}$ & $\begin{array}{l}\text { Berufl. Zukunft } \\
\text { Ausland/offen } \\
\text { Korrelation nach } \\
\text { Pearson }\end{array}$ & Sig. & $\begin{array}{l}\text { Berufl. Zukunft } \\
\text { Ausland/offen } \\
\text { Korrelation nach } \\
\text { Spearmann-Rho }\end{array}$ & Sig. & N \\
\hline $\begin{array}{l}\text { Kommunikation und } \\
\text { Kontakt mit } \\
\text { Deutschen }\end{array}$ & -0.158 & $0.04^{*}$ & -0.224 & $0.003^{* *}$ & 174 \\
\hline $\begin{array}{l}\text { Kommunikation und } \\
\text { Kontakt mit } \\
\text { Menschen aus dem } \\
\text { Herkunftsland in D. }\end{array}$ & 0.097 & 0.23 & 0.095 & 0.23 & 170 \\
\hline $\begin{array}{l}\text { Kommunikation und } \\
\text { Kontakt mit } \\
\text { Menschen aus } \\
\text { anderen Staaten in D. }\end{array}$ & 0,169 & $0.04^{*}$ & 0.16 & $0.056^{*}$ & 158 \\
\hline $\begin{array}{l}\text { Kontakte, Reisen und } \\
\text { Projekte im } \\
\text { Herkunftsland }\end{array}$ & 0,064 & 0.41 & 0.058 & & 143 \\
\hline
\end{tabular}

Quelle: MOBIL 2012/2013; Signifikanzlevel: *p $\leq 0,1, * * \mathrm{p} \leq 0,01, * * * \mathrm{p} \leq 0,001$

Abschließend wird der Zusammenhang zwischen der Ausübung internationaler Aktivitäten und der Bleibeabsicht betrachtet. Je stärker die eigene internationale Mobilitätserfahrung ausgeprägt ist, desto häufiger erwägen Professoren, auch in Zukunft wieder aus beruflichen Gründen ins Ausland zu wechseln. Auch im Falle einer ausgeprägten internationalen Publikationstätigkeit erweist sich diese Wahrscheinlichkeit als besonders hoch. Der stärkste Zusammenhang zwischen internationaler Mobilität und Bleibeabsicht zeigt sich bei Professoren, die häufig in nichtdeutscher Sprache Forschung und Lehre betreiben. Eine starke internationale Ausrichtung in diesem Bereich korreliert in hohem Maße mit der Bereitschaft, die berufliche Zukunft im Ausland zu verbringen (vgl. Tabelle 6.16). Der Index zur 
nichtdeutschen Lehre und Forschung basiert primär auf einer englischsprachigen Ausrichtung in beiden Feldern (vgl. Abschnitt 6.6.4). Ein Erklärungsansatz könnte sein, dass der „routinierte“ Umgang in Forschung und Lehre mit der englischen Sprache dazu beiträgt, dass Professoren sich eher zutrauen, Professorenstellen mit englischsprachigem Profil im Ausland nachzugehen.

Tabelle 6.16 Unterschiede hinsichtlich der Bleibeabsicht nach internationalen Aktivitäten

\begin{tabular}{|c|c|c|c|c|c|}
\hline $\begin{array}{l}\text { Index berufl. } \\
\text { Internationale } \\
\text { Aktivitäten von nie } \\
\text { (1) bis oft (3) }\end{array}$ & $\begin{array}{l}\text { Berufl. } \\
\text { Zukunft } \\
\text { Ausland/offen } \\
\text { Korrelation } \\
\text { nach Pearson }\end{array}$ & Sig. & $\begin{array}{l}\text { Berufl. Zukunft } \\
\text { Ausland/offen } \\
\text { Korrelation } \\
\text { nach } \\
\text { Spearman-Rho }\end{array}$ & Sig. & $\mathrm{N}$ \\
\hline $\begin{array}{l}\text { Internationale } \\
\text { Mobilität und } \\
\text { Auslandserfahrung }\end{array}$ & 0.274 & $0.001 * * *$ & 0.267 & $0.002 * *$ & 139 \\
\hline $\begin{array}{l}\text { Internationale } \\
\text { Publikationen }^{\mathrm{a}}\end{array}$ & 0.147 & $0.06^{*}$ & 0.13 & $0.097 *$ & 165 \\
\hline $\begin{array}{l}\text { Tätigkeiten im } \\
\text { Ausland }^{\mathrm{a}}\end{array}$ & 0.188 & 0.13 & 0.106 & 0.17 & 170 \\
\hline $\begin{array}{l}\text { Internationale } \\
\text { Kooperationen }^{\mathrm{a}}\end{array}$ & 0.06 & 0.46 & 0.07 & 0.42 & 140 \\
\hline $\begin{array}{l}\text { Nichtdeutsche } \\
\text { Sprachen in } \\
\text { Forschung und } \\
\text { Lehre }^{c}\end{array}$ & 0.276 & $<0.000 * * *$ & 0.275 & $<0.000 * * *$ & 174 \\
\hline $\begin{array}{l}\text { Interkulturelle } \\
\text { berufl. Tätigkeiten }\end{array}$ & 0.121 & 0.121 & 0 & 0.102 & 169 \\
\hline
\end{tabular}

Quelle: MOBIL 2012/2013; Signifikanzlevel: *p $\leq 0,1, * * p \leq 0,01, * * * p \leq 0,001$. ${ }^{a}$ Anzahl unterschiedlicher Formen ${ }^{\mathrm{b}}$ Index von $0-5^{\mathrm{c}}$ Index von 1 bis 3

Abschließend wird über eine logistische Regressionsanalyse der multivariate Einfluss der wichtigsten unabhängigen Variablen untersucht. Hinsichtlich der soziodemographischen Merkmale zeigen sich die größten Unterschiede je nach Aufenthaltsdauer in Deutschland, Alter und Besoldungsgruppe. Aufgrund der hohen Kollinearität zwischen Alter und Besoldungsgruppe werden hier ausschließlich die Besoldungsgruppen berücksichtigt, da sich für diese Variable noch größere Unterschiede zeigen. Zugleich wurde geprüft, ob die Unterschiede zwischen den Gruppen der Aufenthaltsdauer evtl. auf die unterschiedliche Altersstruktur zurückzuführen ist (vgl. Abschn. 6.1.3). Die Analyse zeigt, dass die 
Unterschiede nach Aufenthaltsdauer zur Zukunftsplanung im Ausland auch unter Kontrolle der Altersstruktur konstant bleiben. Als weitere beeinflussende Variablen werden die berufliche Zufriedenheit und das Herkunftsland des Partners betrachtet. Zudem wurde für die Modellbildung die private Interaktion mit Deutschen als wichtigste Variable für private Interaktionen berücksichtigt und Forschung und Lehre in nichtdeutscher Sprache als wichtigste Variable für internationale berufliche Aktivitäten.

Dabei wird erneut eine schrittweise Modellbildung nach dem Konzept von Long und Freese (2014) vorgenommen (vgl. Abschnitt 6.5). Die Entwicklung der Modellgüte wird dabei über das „Akaike's information criterion“ (AIC) und das „Bayesian information criterion“ (BIC) sowie Pseudo- $\mathrm{R}^{2}$ bestimmt. Während in Bezug auf AIC und BIC ein kleinerer Wert auf eine bessere Modellgüte hinweist, bedeutet für Pseudo- $\mathrm{R}^{2}$ ein Anstieg des Wertes eine Verbesserung der Modellgüte (vgl. ausführlich Long und Freese 2014, S. 122-124).

Bei den ersten vier Variablen zeigt sich eine konstante Verbesserung der Modellgüte. Insbesondere die berufliche Zufriedenheit und das Herkunftsland des Partners führen dabei zu einer deutlichen Verbesserung der Modellgüte (vgl. Tabelle 6.17). Als fünfte Variable wurde die Interaktion mit Deutschen aufgenommen. Da die Modellgüte durch diese Variable verringert wurde, wird sie im abschließenden Regressionsmodell nicht berücksichtigt. Durch die Variable Lehre und Forschung in nichtdeutscher Sprache konnte abschließend eine geringfügige Verbesserung hergestellt werden. ${ }^{30}$

Die Ergebnisse zeigen, dass die Aufenthaltsdauer in Deutschland auch multivariat einen wichtigen Prädikator für die berufliche Zukunftsplanung darstellt, und zwar unabhängig von den anderen Einflussvariablen. Die Besoldungsgruppe hingegen hat zwar bivariat einen signifikanten Einfluss, die Unterschiede verringern sich aber deutlich und sind nicht mehr signifikant, sobald sie in Verbindung mit der beruflichen Zufriedenheit betrachtet werden. Das heißt die Unterschiede zwischen den Besoldungsgruppen hinsichtlich der Zukunftsplanung im Ausland lassen sich zu einem großen Teil damit erklären, dass die Besoldungsgruppen unterschiedlich zufrieden sind (vgl. Tabelle 6.17: Model 3). Die deutlich geringere Wahrscheinlichkeit, dass Fachhochschulprofessoren ins Ausland wechseln, ist auch damit zu erklären, dass der Arbeitsalltag weniger international gestaltet ist. Eine Kontrolle nach dem Indikator nichtdeutsche Sprachen in Lehre und Forschung zeigt, dass die Unterschiede zwischen Fachhochschulprofessoren und den

\footnotetext{
${ }^{30}$ Vergleichend wurde auch die Variable „Internationale Mobilität und Auslandserfahrung“ in das Modell aufgenommen. Unter anderem aufgrund der hohen Zahl an „Missings“ (vgl. Tabelle 6.15) konnte die Modellgüte nicht verbessert werden.
} 
Tabelle 6.17 Binäre logistische Regression über Einflussfaktoren auf eine berufliche Zukunftsplanung im Ausland/offen (average marginal effects)

\begin{tabular}{|c|c|c|c|c|c|}
\hline & Model 1 & $\begin{array}{l}\text { Model } \\
2\end{array}$ & Model 3 & Model 4 & Model 5 \\
\hline \multicolumn{6}{|l|}{$\begin{array}{l}\text { Aufenthaltsdauer in Deutschland } \\
\text { (Ref.: Max. } 10 \text { Jahre in } \\
\text { Deutschland) }\end{array}$} \\
\hline Zwischen 11-20 Jahren & $-0.36 * *$ & $-0.30 *$ & $-0.31 * *$ & $-0.32 * * *$ & $-0.31 * *$ \\
\hline Über 20 Jahre in Deutschland & $-0.32 * *$ & $-0.26^{*}$ & $-0.28 * *$ & $-0.25 * *$ & $-0.23 *$ \\
\hline Zweite Generation & -0.09 & -0.05 & -0.09 & -0.04 & -0.05 \\
\hline \multicolumn{6}{|l|}{$\begin{array}{l}\text { Besoldungsgruppen/Hochschulart } \\
\text { (Ref.: Juniorprofessur) }\end{array}$} \\
\hline Fachhochschulprofessur & & $-0.28 *$ & -0.18 & -0.13 & -0.08 \\
\hline W2-/C3-/C2-Professur (Uni) & & -0.1 & -0.02 & 0.04 & 0.06 \\
\hline W3-/C4-Professur (Uni) & & -0.19 & -0.05 & -0.01 & -0.003 \\
\hline \multicolumn{6}{|l|}{$\begin{array}{l}\text { Berufliche Zufriedenheit (Ref.: } \\
\text { Überhaupt nicht oder eher nicht } \\
\text { zufrieden) }\end{array}$} \\
\hline Teils, teils & & & -0.17 & $-0.24 *$ & -0.22 \\
\hline Eher zufrieden & & & $-0.36^{* * *}$ & $-0.38 * * *$ & $-0.36^{* * *}$ \\
\hline Voll und ganz zufrieden & & & $-0.5^{* * *}$ & $-0.53 * * *$ & $-0.5^{* * *}$ \\
\hline \multicolumn{6}{|l|}{$\begin{array}{l}\text { Herkunft Partner/in (Ref.: } \\
\text { Deutschland) }\end{array}$} \\
\hline Keine Partnerschaft & & & & -0.004 & 0.02 \\
\hline $\begin{array}{l}\text { Partner aus meinem } \\
\text { Herkunftsland }\end{array}$ & & & & $0.21 *$ & $0.2 *$ \\
\hline Partner aus einem anderen Staat & & & & $0.36 * *$ & $0.34 * * *$ \\
\hline $\begin{array}{l}\text { Lehre und Forschung in } \\
\text { nichtdeutscher Sprache (Index) }\end{array}$ & & & & & 0.13 \\
\hline $\mathrm{BIC}$ & 218.9 & 228.9 & 225.2 & 225.2 & 227.8 \\
\hline AIC & 206.5 & 207.2 & 194.3 & 184.9 & 184.5 \\
\hline Pseudo R^2 & 0.08 & 0.1 & 0.19 & 0.26 & 0.27 \\
\hline $\mathrm{N}$ & 163 & 163 & 163 & 163 & 163 \\
\hline
\end{tabular}

Quelle: MOBIL 2012/2013; Signifikanzlevel: *p $\leq 0,1, * * \mathrm{p} \leq 0,01, * * * \mathrm{p} \leq 0,001$

anderen Universitätsprofessoren deutlich zurückgehen (vgl. Tabelle 6.17: Model 5). Die berufliche Zufriedenheit lässt sich als wichtigster Prädikator hervorheben. 
Die Wahrscheinlichkeit, dass Professoren, die eher oder überhaupt nicht zufrieden sind, erwägen, ins Ausland zu wechseln, liegt durchgehend um mindestens 50 Prozentpunkte höher als bei Professoren, die voll und ganz zufrieden sind. Demgegenüber erhöhen Partnerschaften mit Menschen, die nicht aus Deutschland kommen, die Wahrscheinlichkeit, dass die Professoren einen Wechsel ins Ausland erwägen, deutlich.

Die in Abschnitt 6.5 ausführlich dargelegten Befunde über die zentralen Arbeitsbedingungen für die berufliche Zufriedenheit sollten auch dahingehend berücksichtigt werden, dass sie eine hohe Relevanz haben, um Professoren mit Migrationshintergrund langfristig in Deutschland zu halten. Berufliche Zufriedenheit ist zugleich der Aspekt, der am stärksten durch politische Maßnahmen beeinflusst werden kann. Entsprechendes gilt etwa nicht für die Partnerwahl. Allerdings sollte die Erkenntnis hervorgehoben werden, dass sowohl private Kontakte und Kommunikation zu Deutschen als auch die Partnerschaft zu einer Person aus Deutschland unmittelbar mit der beruflichen Zukunftsplanung zusammenhängen. Dies bedeutet, dass auch die private Situation und Teilhabe eine zentrale Rolle für die Zukunftsplanung in Deutschland spielen. Darüber hinaus sollte - wie bereits kurz angedeutet - näher untersucht werden, warum es insbesondere in der zweiten Generation von Professoren mit Migrationshintergrund eine so große Gruppe gibt, die einen Wechsel ins Ausland erwägt, da selbst unter Berücksichtigung der größeren Unzufriedenheit die Bleibeabsichten deutlich geringer ausfallen als bei zugewanderten Professoren, die bereits über zehn Jahre in Deutschland leben.

\subsubsection{Nachberufliche Zukunftsplanung}

Neben den beruflichen Zukunftsplänen wurde auch untersucht, inwieweit Professoren mit Migrationshintergrund ihre nachberufliche Zukunft in Deutschland planen. Die Frage ist natürlich für die jüngere Generation deutlich schwerer zu beantworten als für Professoren, die bereits kurz vor der Pensionierung stehen. Dementsprechend verwundert es wenig, dass fast die Hälfte der jüngeren Generation $(48 \%)$ und der mittleren Generation $(43 \%)$ die Frage offen lässt, während lediglich ein Viertel der älteren Generation sich diesbezüglich noch nicht festlegen will (vgl. Abbildung 6.54).

Insgesamt plant knapp ein Viertel der Professoren die nachberufliche Zukunft im Ausland, während deutlich über ein Drittel definitiv plant, auch nach dem Berufsleben in Deutschland zu bleiben. Einen wichtigen Einflussfaktor stellt dabei 


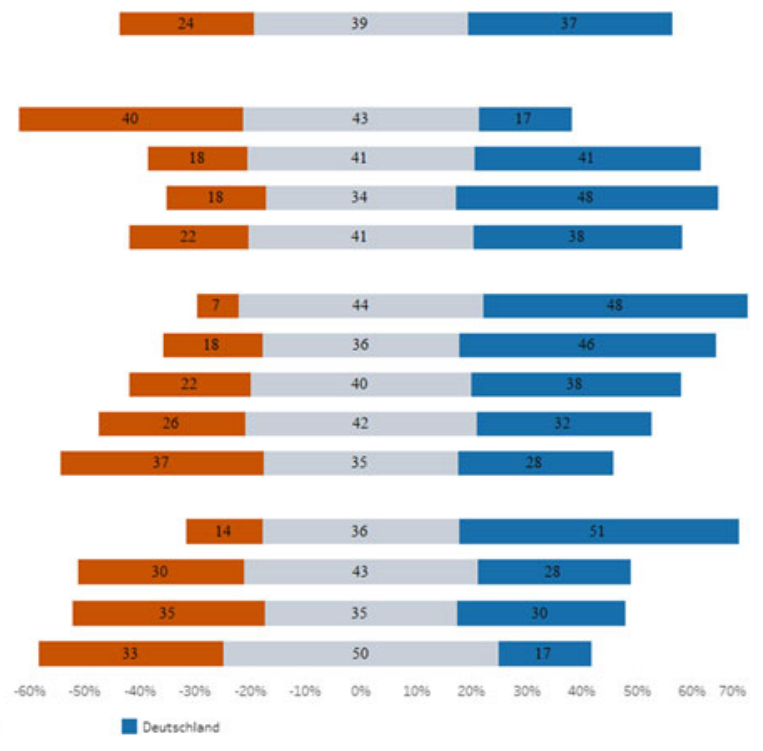

Max. 10 Jahre in Deutschland

Zwischen 11-20 Jahren

Ober 20 Jahre in Deutschland

Zweite Generation

Osteuropa

Angelsachsische Lander*

Weitere Lander Westeuropas

Lateinamerika, Asien und Afrika

Osterreich und Schweiz

Aus Deutschland

Aus meinem Herkunftsland

Keine Partnerschaft

Aus einem anderen Staat

Eusiond

Nexh often

IIt Nochoffen

Abbildung 6.54 Nachberufliche Zukunftspläne nach Aufenthaltsdauer in Deutschland, Herkunftsregion und Herkunft Partner. (Quelle: MOBIL 2012/2013 (Gesamt: N = 182, Dauer in Deutschland: $\mathrm{N}=179(\mathrm{p}=0,004)$, Herkunftsregion: $\mathrm{N}=181(\mathrm{p}=0,062)$, Herkunft Partner: $\mathrm{N}=174(\mathrm{p}=0,015), \mathrm{p}$-value basiert auf Kruskal-Wallis-Test. *Kanada, USA, Australien)

die Aufenthaltsdauer in Deutschland dar. Während ein Großteil der Professoren, die erst seit zehn Jahren in Deutschland leben, plant, Deutschland nach der Berufstätigkeit wieder zu verlassen, wollen über $40 \%$ der Personen, die bereits länger hier sind, auch nach dem Berufsleben in Deutschland bleiben. Eine Sonderstellung nimmt wie auch bei der beruflichen Zukunftsplanung die zweite Generation ein, wobei hier die Emigrationspläne im Vergleich etwas weniger stark ausgeprägt sind als bei den beruflichen Zukunftsplänen und auch in Verbindung mit diesen zu erklären sind. Auffällige Unterschiede zeigen sich ebenfalls hinsichtlich der Herkunftsregionen. Speziell Professoren aus Osteuropa und den angelsächsischen Ländern planen, auch nach der Berufstätigkeit in Deutschland zu bleiben. Demgegenüber planen insbesondere Professoren aus der Schweiz und Österreich, Deutschland nach dem Berufsleben wieder zu verlassen. Dabei ist zu berücksichtigen, dass insbesondere Professoren aus diesen beiden Ländern im Durchschnitt vergleichsweise kurz in Deutschland leben. Als zentraler Faktor 
erweist sich zudem das Herkunftsland des Partners. Über die Hälfte der Professoren, die eine feste Partnerschaft mit einem Menschen aus Deutschland haben, plant ihre nachberufliche Zukunft definitiv in Deutschland (vgl. Abbildung 6.54).

Abschließend soll noch ein Blick auf die möglichen Zielländer der beruflichen oder nachberuflichen Zukunft geworfen werden. Aufgrund der geringen Fallzahlen sollten diese nur als Tendenzen verstanden werden. Zwei Beobachtungen sollten dennoch festgehalten werden. Hinsichtlich der möglichen Zielländer für die berufliche Zukunft spielen die USA, die Schweiz und das Vereinigte Königreich eine zentrale Rolle, wobei die USA das mit Abstand beliebteste Zielland darstellen. Diesbezüglich lässt sich der Schluss ziehen, dass Deutschland insbesondere mit diesen drei Ländern im Wettbewerb um hochqualifiziertes wissenschaftliches Personal steht. Zu berücksichtigen ist dabei aber auch, dass die Befragung vor der Wahl Donald Trumps zum Präsidenten der Vereinigten Staaten und dem Brexit-Referendum durchgeführt wurde. Es wäre interessant, zu untersuchen, inwieweit die damals geäußerten Präferenzen aus heutiger Sicht noch Bestand haben. Ferner zeigt sich bei den Zielländern der nachberuflichen Zukunft eine größere Diversität, die in deutlich stärkerem Maße der Herkunftsländerstruktur der Professoren entspricht (vgl. Abbildung 6.55).

Zielländer berufliche Zukunft

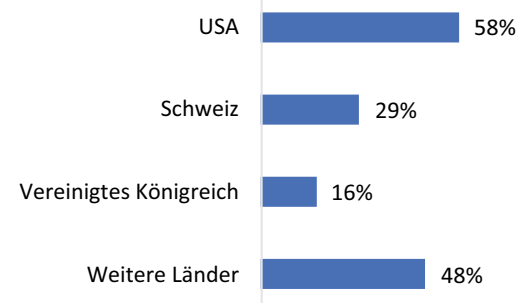

Zielländer nachberufliche Zukunft

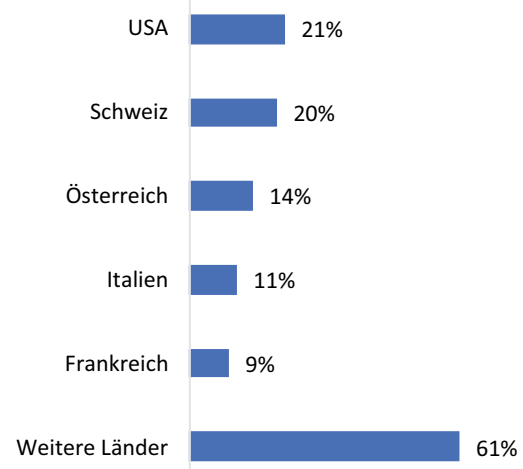

Abbildung 6.55 Mögliche Zielländer der langfristigen beruflichen/nachberuflichen Zukunft. (Quelle: MOBIL 2012/2013; Berufliche Zukunft N = 31; Nachberufliche Zukunft $\mathrm{N}=56$ [Mehrfachantworten]) 


\subsection{Integration, soziale Teilhabe und Transnationalität}

Im folgenden Kapitel wird das Integrationsverständnis nach Pries (2015) zugrunde gelegt. Der Ansatz grenzt sich sowohl von assimilationstheoretischen Vorannahmen ab als auch vom Konzept des ethnischen Pluralismus, sondern versteht Integration als ergebnisoffenen sozialen Prozess, der sich nach ökonomischer, kultureller, politischer und sozialer Ebene differenzieren lässt. Zugleich beschränkt sich das Verständnis nicht ausschließlich auf Nationalgesellschaften, sondern basiert auf einem transnationalen Teilhabeverständnis, das auch Prozesse über nationalstaatliche Grenzen hinaus -beispielsweise im Herkunftsland der Migranten - mit in den Blick nimmt (vgl. ausführlich Abschnitt 3.2.2.4). Im Mittelpunkt der folgenden Analyse steht die soziale Teilhabe von Professoren mit Migrationshintergrund in Deutschland (Abschnitt 8.1-8.2) sowie im Herkunftsland (Abschnitt 8.3). Anhand der privaten Kontakte und der Kommunikationssprachen wird untersucht, wie stark die Kontakte zu Deutschen, zu Menschen aus dem Herkunftsland und zu Menschen aus weiteren Ländern in Deutschland ausgeprägt sind. Auf dieser Grundlage erfolgt eine Clusterbildung, um unterschiedliche Teilhabemuster herauszuarbeiten. Abschließend werden Verbindungen und Teilhabe im Herkunftsland selbst analysiert.

\subsubsection{Kontakte und Kommunikation zu unterschiedlichen Gruppen in Deutschland}

Um soziale Teilhabe von Menschen mit Migrationshintergrund zu untersuchen, lassen sich grundsätzlich eine Vielzahl von Feldern, wie Wohnen, soziale Beziehungen, Familie, Heiratskreise und Identität, in den Blick nehmen (vgl. Abschnitt 3.2.2.4). Die folgenden Ausführungen beziehen sich auf das Feld der sozialen Beziehungen in Deutschland. Dabei wird zwischen privaten Kontakten und Kommunikation zu Deutschen, zu Menschen aus dem Herkunftsland und zu Menschen aus weiteren Ländern unterschieden. Operationalisiert wurde die Teilhabe in Bezug auf die drei Gruppen anhand von drei Items. Auf häufige private Kontakte zu Deutschen und auf die häufige Nutzung des Deutschen als Kommunikationssprache im Freundeskreis verweisen jeweils über $90 \%$ der Professoren mit Migrationshintergrund. Bei etwas unter $90 \%$ liegt der Anteil derer, die Deutsch häufig zur Kommunikation in der Familie verwenden (vgl. Abbildung 6.56).

Während der Kontakt und die Kommunikation mit Deutschen für fast alle Professoren mit Migrationshintergrund integraler Bestandteil des Alltags ist, zeigen sich bei der Interaktion mit Menschen aus dem Herkunftsland deutlich größere 
Unterschiede. Weniger als die Hälfte hat in Deutschland häufig privaten Kontakt zu Menschen aus dem Herkunftsland. In diesem Kontext stellt sich die Frage, inwieweit möglicherweise neben der aktiven Entscheidung, gezielt private Kontakte zu Menschen aus dem Herkunftsland zu pflegen, auch Gelegenheitsstrukturen dahingehend eine Rolle spielen, wie groß die „Herkunftsland-Community“ in Deutschland und in der Wissenschaft ist. Je nach Herkunftsland zeigen sich allerdings nur geringfügige Unterschiede. Die Sprache des Herkunftslandes verwenden knapp $60 \%$ der Befragten häufig im Freundeskreis. Am häufigsten findet die Kommunikation in der Herkunftssprache im Rahmen der Familie statt. Dies gilt für über zwei Drittel der Befragten (vgl. Abbildung 6.56).

\section{Interaktion mit Deutschen}

Nutzung Deutsch im Freundeskreis

Nutzung Sprache Deutsch in der Familie

Private Kontakte in Deutschland mit Deutschen

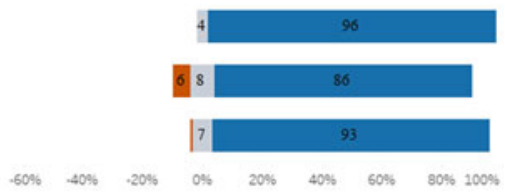

Interaktion mit Bezug zum Herkunftsland

Nutzung Sprache des Herkunftslandes im Freundeskreis

Nutzung Sprache des Herkunftslandes in der Familie

Private Kontakte in Deutschland mit Menschen aus Herkunftsland

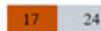

$13 \quad 18$

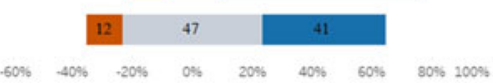

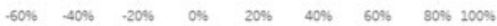

Interaktion mit Bezug zu weiteren Ländern

Nutzung anderer Sprachen in der Familie

Nutzung weiterer Sprachen im Freundeskreis

Private Kontakte in Deutschland mit Menschen aus weiteren Landern
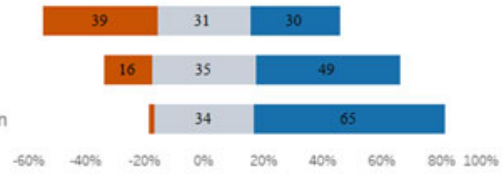

Nie

Welten

Q Haufig

Abbildung 6.56 Private Kontakte und Kommunikation in Deutschland. (Quelle: MOBIL 2012/2013, Item 1a $N=175$, Item 1b: $N=180$, Item 1c $N=181 ; 2 \mathrm{a} \mathrm{N}=173,2 \mathrm{~b} \mathrm{~N}=167$, $2 \mathrm{c}=161 ; 3 \mathrm{a} \mathrm{N}=172,3 \mathrm{~b} \mathrm{~N}=129,3 \mathrm{c} \mathrm{N}=148$ )

Hinsichtlich der Interaktion zu Menschen aus weiteren Ländern zeigt sich im Vergleich zur Interaktion mit Menschen aus dem Herkunftsland eine gegenläufige Verteilung. Am seltensten werden weitere Sprachen in der Familie verwendet. In 
etwa die Hälfte verwendet häufig weitere Sprachen im Freundeskreis und etwa zwei Drittel pflegen häufige private Kontakte zu Menschen aus weiteren Ländern (vgl. Abbildung 6.56).

In einem zweiten Schritt wurde ein Mittelwertindex hinsichtlich der Interaktion mit den drei Gruppen gebildet ${ }^{31}$, über den die Interaktion zwischen 1 (Nie), 2 (Selten) und 3 (Häufig) dargestellt wird. Der Index für die Interaktion mit Deutschen fällt mit Abstand am höchsten aus und liegt damit vor der Interaktion mit Menschen aus dem Herkunftsland sowie der Interaktion mit Menschen aus weiteren Ländern (vgl. Tabelle 6.18).

Tabelle 6.18 Index zur Interaktion mit Deutschen, Menschen aus dem Herkunftsland und Menschen aus weiteren Ländern

\begin{tabular}{l|l|l}
\hline INDEX & Mean & SD \\
\hline Interaktion mit Deutschen & 2,89 & 0,25 \\
\hline Interaktion mit Menschen aus dem Herkunftsland & 2,43 & 0,58 \\
\hline Interaktion mit Menschen aus weiteren Ländern & 2,33 & 0,56 \\
\hline
\end{tabular}

Quelle: MOBIL 2012/2013 (INDEX 1: $\mathrm{N}=182$, INDEX 2: $\mathrm{N}=169$, INDEX 3: $\mathrm{N}=152$ )

Bei der Interaktion mit Deutschen zeigen sich signifikante Unterschiede zwischen Early Migrants (3,0), Student Migrants (2,9) und Professional Migrants $(2,8)$. Zugleich wird ein signifikanter Zusammenhang mit der Aufenthaltsdauer der Professoren in Deutschland sichtbar. Auffällig ist zudem die etwas geringere Interaktion von Professoren aus Entwicklungs- und Schwellenländern $(2,8)$ mit Deutschen. Dennoch bleibt festzuhalten, dass fast alle Professoren mit Migrationshintergrund häufig Kontakt und Kommunikation zu Deutschen haben.

Erwartungsgemäß zeigen sich hinsichtlich der Interaktion zum Herkunftsland gegenläufige Unterschiede zwischen den Migrationstypen. Professional Migrants $(2,6)$ haben am häufigsten Kontakt zu Menschen aus dem Herkunftsland. Damit liegen sie sowohl vor den Student Migrants $(2,4)$ als auch deutlich vor den Early Migrants (2,2). Auch hier besteht vermutlich ein Zusammenhang zur Aufenthaltsdauer, nach der sich ebenfalls Unterschiede zeigen.

Überraschend bei der Interaktion zu Deutschen und zu Menschen aus dem Herkunftsland sind weniger die Unterschiede zwischen den Migrationstypen als vielmehr der Befund, dass einerseits Professional Migrants, die erst seit Kurzem in Deutschland sind, bereits in hohem Maße Kontakte zu Deutschen haben

\footnotetext{
${ }^{31}$ Ein Mittelwert wurde gebildet, wenn mindestens zwei der drei Items beantwortet wurden.
} 
und in deutscher Sprache kommunizieren und andererseits Early Migrants und die zweite Einwanderergeneration weiterhin in besonderem Maße Interaktionen zu Menschen aus dem Herkunftsland aufrechterhalten. Bei den Professional Migrants lassen sich die hohen Anteile relativ einfach über den überdurchschnittlich hohen Anteil von Professoren aus Österreich und der Schweiz erklären. Um diese Interaktionsformen noch grundlegender nachvollziehen zu können, sollten in zukünftigen Studien auch Gelegenheitsstrukturen berücksichtigt werden. Es lässt sich vermuten, dass der Kontakt und die Kommunikation zu Menschen aus dem Herkunftsland in Deutschland auch in hohem Maße damit zusammenhängen, wie stark Menschen aus dem Herkunftsland in der jeweiligen Region sowie im spezifischen Milieu und Berufsfeld vertreten sind. Bei den Kontakten zu Menschen aus weiteren Ländern zeigen sich keine Unterscheide zwischen den Migrationstypen.

Zusammenfassend lässt sich festhalten, dass sowohl Kommunikation und Kontakte mit Deutschen als auch mit Menschen aus dem Herkunftsland und aus weiteren Ländern in sehr hohem Maße bei Professoren mit Migrationshintergrund stattfinden. In zukünftigen Studien sollten die Fragestellungen noch etwas differenzierter erhoben werden. So stellt sich beispielsweise das Problem, dass die Herkunftssprache und die deutsche Sprache für den Großteil der Österreicher und Schweizer vermutlich identisch ist. Bei den weiteren Sprachen ist zu vermuten, dass es sich hier primär um die englische Sprache handelt, womit zu erklären ist, dass weitere Sprachen von Professoren aus angelsächsischen Ländern leicht unterdurchschnittlich verwendet werden. Eine interessante offene Frage ist der Zusammenhang der drei Formen privater Kontakte, der im Folgenden näher untersucht wird.

\subsubsection{Cluster zu interkulturellen Interaktionen}

Eine der großen Kontroversen in der Migrationsforschung liegt in der Frage, inwieweit eine Mehrfachintegration oder -teilhabe möglich ist. So vertritt beispielsweise Esser die These, dass eine Mehrfachintegration sowohl in die Einwanderungsgesellschaft als auch in die ethnische Gemeinschaft aufgrund der hohen Anforderungen unwahrscheinlich sei (vgl. Abschnitt 3.2.2.1). Demgegenüber wird aus der transnationalen Perspektive Mehrfachzugehörigkeit zu mehreren nationalkulturellen Kontexten vielmehr als Normalform migrantischer Lebensweisen verstanden (vgl. 3.2.2.1).

Im Folgenden soll für die Gruppe der Professoren mit Migrationshintergrund untersucht werden, inwieweit im beschriebenen Bereich der sozialen Teilhabe 
Professoren sich primär exklusiv auf Kommunikation und Kontakte mit einzelnen Gruppen beschränken oder ob sich häufige Kommunikation und Kontakte auch gleichzeitig zu mehreren Gruppen zeigen. Um dieser Frage nachzugehen, wird auf Grundlage der dargestellten Indexe über die jeweilige Interaktion mit den drei Gruppen eine Clusteranalyse durchgeführt, um die unterschiedlichen Interaktionstypen zu identifizieren.

Die Clusteranalyse ist eine explorative multivariate Methode, um Personen anhand mehrerer Merkmale (Variablen) in Gruppen (Clustern) zusammenzufassen. Ziel des Verfahrens ist es, dass die Personen innerhalb eines Clusters möglichst homogen sind und die gebildeten Cluster sich zugleich möglichst stark voneinander unterscheiden (Janssen und Laatz 2017).

Dabei wurde eine Two-Step-Clusteranalyse mithilfe von SPSS Statistics 25 durchgeführt. Als Distanzmaß wurde Log-Likelihood und als Clusterkriterium das „Bayesian information criterion“ (BIC) verwendet. Der erste Schritt besteht darin, die Fälle sequentiell abzuarbeiten und sukzessive Sub-Cluster mit jeweils ähnlichen Fällen zu bilden. In einem zweiten Schritt werden die Sub-Cluster über agglomerative hierarchische Clusteranalysen zu End-Clustern zusammengefasst. Die Anzahl der Cluster wird bei der Two-Step-Clusteranalyse nicht vorgegeben, sondern über das Verfahren ,automatisch“ bestimmt. Ferner wird für jede Clusterlösungssequenz das BIC berechnet und auf dieser Grundlage die Obergrenze für eine optimale Clusteranzahl bestimmt. Abschließend wird mithilfe eines zweiten Auswahlkriteriums die Lösung optimiert. Hinsichtlich der Vorannahmen zur Unabhängigkeit der Variablen ${ }^{32}$ und zur Normalverteilung der Variablen lässt sich die Two-Step-Clusteranalyse nach SPSS als ein robustes Verfahren beschreiben, das nicht sehr empfindlich auf Verletzungen der Vorannahmen reagiert (Janssen und Laatz 2017).

Die Ergebnisse der Two-Step-Clusteranalyse basieren auf den gebildeten Indexen „Interaktion mit Deutschen“, „Interaktion mit Menschen aus dem Herkunftsland“ und „Interaktion mit Menschen aus weiteren Ländern“. Die Analyse führt zu einer 4-Clusterlösung (vgl. Abbildung 6.57), deren Qualität auf der Grundlage des Silhouettenkoeffizienten für Kohäsion und Separation $(0,5)$ als zufriedenstellend einzuordnen ist. Das Größenverhältnis zwischen dem kleinsten Cluster $(\mathrm{n}=18)$ und dem größten Cluster $(\mathrm{n}=54)$ lässt sich ebenfalls als akzeptabel beschreiben. Der Prädikator zum Index „Interaktion mit Deutschen“ $(1,0)$ hat den stärksten Einfluss auf die Clusterbildung, vor dem Index „Interaktion mit

\footnotetext{
${ }^{32}$ Lediglich zwischen den Indexen „Interaktion mit Menschen aus dem Herkunftsland“ und „Interaktion mit Menschen aus weiteren Ländern“ zeigt sich eine schwache signifikante Korrelation von $r=0,174$.
} 
Menschen aus weiteren Ländern“ $(0,63)$ und dem Index „Interaktion mit Menschen aus dem Herkunftsland“ (0,56). Die Unterschiede der Indexwerte zwischen den Clustern sind durchgehend hoch signifikant (vgl. Tabelle 6.19).

Die durchgeführte Two-Step-Clusteranalyse führt zu einer 4-Clusterlösung, die im Folgenden interpretiert wird. Dabei wurde geprüft, inwieweit für jedes Cluster der jeweilige Indexwert deutlich über dem Durchschnitt liegt (vgl. Tabelle 6.19).

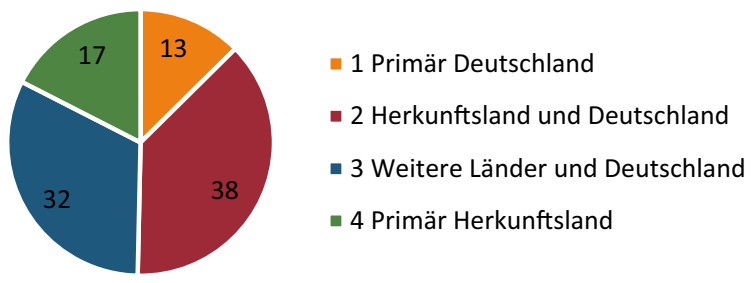

Abbildung 6.57 Cluster zur sozialen Interaktion in Deutschland mit unterschiedlichen Gruppen. (Quelle: MOBIL 2012/2013 N = 143)

Für das erste Cluster fällt der Indexwert für die Interaktion mit Deutschen $(2,96)$ deutlich höher aus als im Durchschnitt $(2,88)$. Demgegenüber liegen die Indexwerte für Interaktion mit Menschen aus dem Herkunftsland und aus weiteren Ländern deutlich unter dem Durchschnitt. Auf der Grundlage dieser Orientierung bildet das erste Cluster Professoren ab, die primär mit Menschen in Deutschland in Interaktion treten (,,primär Deutschland“). Diese Professoren stellen mit einem Anteil von $13 \%$ das kleinste Cluster dar. Das zweite und größte Cluster umfasst indessen $38 \%$ der Professoren mit Migrationshintergrund. Die Indexwerte für Interaktion mit Deutschen (3,0) und Interaktion mit Menschen aus dem Herkunftsland $(2,65)$ liegen deutlich über dem Durchschnitt, während der Indexwert für Interaktion mit Menschen aus weiteren Ländern unter dem Durchschnitt liegt. Dementsprechend lässt sich das größte Cluster als „Herkunftsland und Deutschland" orientiert beschreiben (vgl. Abbildung 6.57). Analog wurden die beiden weiteren Cluster interpretiert.

Das dritte Cluster umfasst etwa ein Drittel (32\%) der Professoren mit Migrationshintergrund. Dieses Cluster orientiert sich primär an weiteren Ländern und Deutschland. Professoren des vierten Clusters (17\%) treten mit Abstand am häufigsten mit Menschen aus dem Herkunftsland in Interaktion. Zugleich zeigt sich hier eine deutlich unterdurchschnittliche Interaktion mit Deutschen und eine leicht 
unterdurchschnittliche Interaktion mit Menschen aus weiteren Ländern. Dementsprechend lässt sich dieses Cluster als „Herkunftsland-orientiert“ beschreiben (vgl. Abbildung 6.57).

Die Ergebnisse weisen deutlich darauf hin, dass bei der Mehrheit der Professoren mit Migrationshintergrund keine eindeutige exklusive Teilhabe in Bezug auf eine der drei Gruppen stattfindet, sondern dass im Gegenteil über zwei Drittel der Befragten in sehr hohem Maße private Kontakte und Kommunikation zu mehreren Gruppen pflegen. Hinsichtlich der eingangs dargestellten Kontroverse zur Mehrfachintegration zeigt sich dementsprechend für die Gruppe der Professoren mit Migrationshintergrund, dass eine Teilhabe in unterschiedlichen Herkunftsgruppen vielmehr den Normalfall als die Ausnahme darstellt. Es spricht also eindeutig gegen die These von Esser, dass Mehrfachintegration nur in Ausnahmefällen möglich sei, und stärkt hingegen den transnationalen Theorienansatz der Mehrfachintegration als Normalform migrantischer Lebensweisen (vgl. Abschnitt 3.2.2). Offen bleibt dabei selbstverständlich, inwieweit sich diese empirische Beobachtung auch auf Migrantengruppen übertragen lässt, die in weniger „privilegierten“ Berufsfeldern arbeiten.

Unterschiede zwischen den Migrationstypen zeigen sich zwischen den Clustern „Primär Deutschland“ und „Primär Herkunftsland“. Während $78 \%$ des Clusters „Primär Deutschland“ bereits hier geboren oder als Kind zugewandert sind, ist über die Hälfte (56 \%) des Clusters „Primär Herkunftsland“ erst als Professional Migrant nach Deutschland migriert. Bei den Herkunftsregionen ist auffällig, dass über $70 \%$ des Clusters „Primär Deutschland“ und des Clusters „Weitere Länder und Deutschland“ aus dem westeuropäischen Ausland kommen. Eine starke Orientierung sowohl hinsichtlich des Herkunftslandes als auch in Bezug auf Deutschland (Cluster 2) findet sich insbesondere bei Professoren aus den USA, Kanada und Australien. Ca. drei Viertel (74 \%) der Professoren aus den angelsächsischen Ländern lassen sich diesem Cluster zuordnen. Die primäre Orientierung auf das Herkunftsland findet sich in besonderem Maße bei Professoren aus Osteuropa, die zu einem Drittel in diesem Cluster zu finden sind.

Bemerkenswert sind auch die Unterschiede zwischen „Humanities“ und „Science“. Professoren aus den „Humanities“ sind deutlich stärker in Cluster 1 „Primär Deutschland“ (78 \%) und Cluster 3 „Deutschland und international“ (64\%) vertreten, während Professoren aus dem Bereich "Science“ in Cluster 2 „Herkunftsland und Deutschland“ (62\%) und Cluster 4 „Primär Herkunftsland“ (56\%) die Mehrheit bilden.

Relevant hinsichtlich der Kontakte zu den unterschiedlichen Gruppen ist zudem die Herkunft des Partners. Der Großteil der Professoren mit Migrationshintergrund $(87 \%)$ lebt in einer festen Partnerschaft. Bei über der Hälfte 


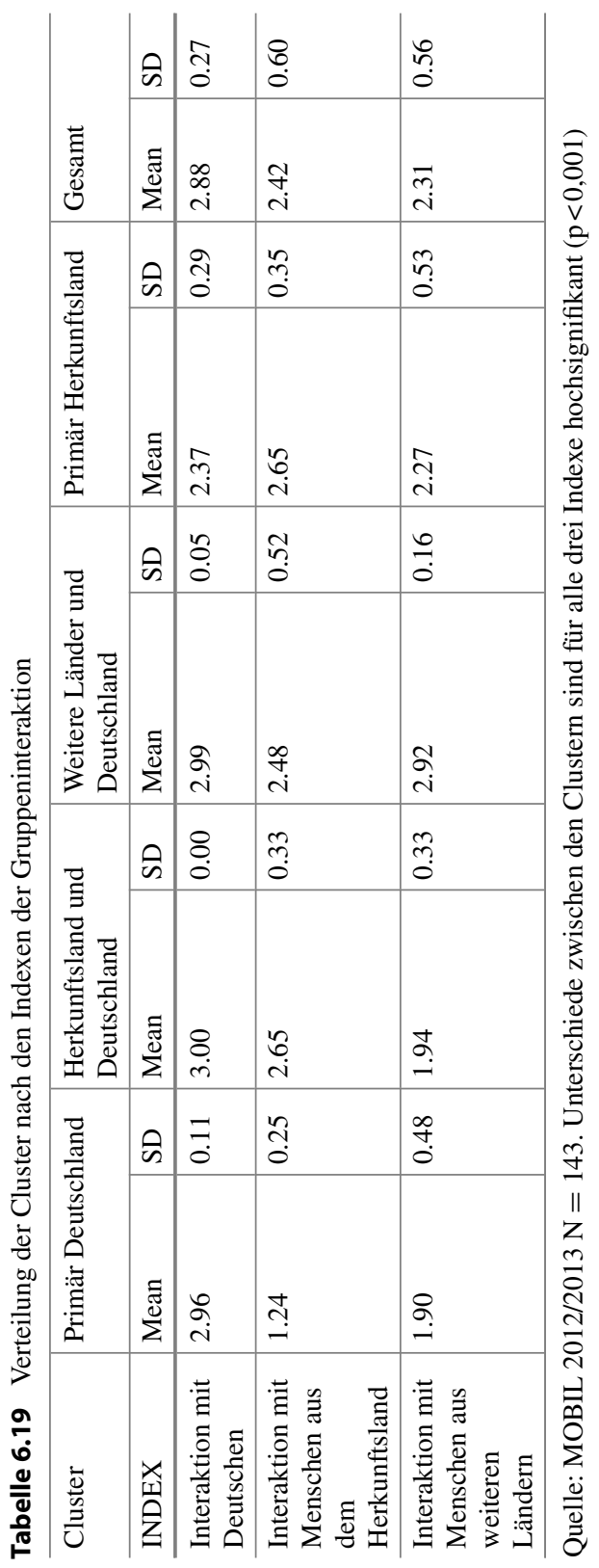


(58 \%) kommt der Partner aus Deutschland, in etwa ein Viertel (26\%) hat einen Partner aus dem Herkunftsland und $16 \%$ der Professoren mit Migrationshintergrund haben einen Partner aus einem anderen Land. Insbesondere bei Cluster 1 und Cluster 4 wird ein starker Zusammenhang sichtbar. Professoren aus dem Cluster „Primär Deutschland“ haben zu 82 \% einen Partner aus Deutschland, während der entsprechende Anteil beim Cluster „Primär Herkunftsland“ bei lediglich $5 \%$ liegt. Professoren in diesem Cluster haben zu $70 \%$ einen Partner aus dem Herkunftsland.

\subsubsection{Verbindungen ins Herkunftsland}

Während der letzte Abschnitt Kontakte und Kommunikation in Deutschland zum Gegenstand hatte, stehen im Folgenden Projekte, Kontakte und Reisen im Herkunftsland im Mittelpunkt.

Knapp die Hälfte der Professoren führt Projekte im Herkunftsland durch. Neben einzelnen unternehmerischen oder sozialen Projekten stehen dabei primär wissenschaftliche Projekte im Vordergrund. Häufigen Kontakt zu Familie und Freunden im Herkunftsland pflegen über zwei Drittel der Professoren mit Migrationshintergrund, während fast die Hälfte häufig in ihr Herkunftsland reist (vgl. Abbildung 6.58).

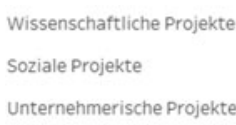

Wissenschaftliche Projekte
Soziale Projekte
Unternehmerische Projekte

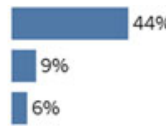

Kontakt zu Familien und Freunden im Herkunftsland Reisen in das Herkunftsland

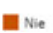

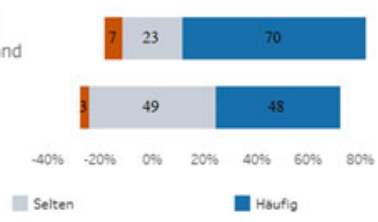

Abbildung 6.58 Projekte, Kontakte und Reisen im Herkunftsland. (Quelle: MOBIL 2012/2013: Projekte $N=154$, Kontakte: $N=174$, Reisen: $N=173$ )

Auf der Grundlage der drei Items wird ein Mittelwertindex gebildet, der zwischen 1 (Keine Projekte, Kontakte oder Reisen) und 3 (Projekte, häufige Kontakte und Reisen) variiert. Die Unterstützung von Projekten wurde dabei so codiert, dass ihr der Wert 3 zugewiesen wird, wenn ein Projekt durchgeführt wird, und der Wert 1, wenn kein Projekt unterstützt wird. Der Indexwert wurde berechnet, wenn mindestens zwei der drei Items beantwortet wurden. 
Der Index liegt für alle Professoren mit Migrationshintergrund bei 2,36. Auffällige Unterschiede zeigen sich auch zwischen Deutschen mit Migrationshintergrund $(2,23)$ und ausländischen Professoren $(2,50)$. Dabei stellt sich die Frage, inwieweit die Unterschiede primär darauf zurückzuführen sind, dass der Ausländeranteil unter Student Migrants und Professional Migrants höher ist als unter Early Migrants. Um dieser Frage nachzugehen, wurde mithilfe von SPSS eine two-way between-groups ANOVA durchgeführt. Durch diese Form der Varianzanalyse zeigt sich ein signifikanter Interaktionseffekt zwischen den Migrationstypen und der Staatsangehörigkeit. Interessant ist dabei, dass der Indexwert bei den Early Migrants für Professoren mit ausländischer Staatsangehörigkeit $(2,66)$ deutlich höher ausfällt als für deutsche Professoren mit Migrationshintergrund (1,98). Demgegenüber liegt der Indexwert für Student Migrants und Professional Migrants bei deutschen Professoren mit Migrationshintergrund sogar etwas höher als bei ausländischen Professoren.

Zudem empfiehlt es sich, die Gruppe der Deutschen mit Migrationshintergrund noch etwas differenzierter zu betrachten. Fast die Hälfte der deutschen Professoren mit Migrationshintergrund (43\%) hat neben der deutschen Staatsangehörigkeit auch die Staatsangehörigkeit des Herkunftslandes. Durch einen Vergleich dieser beiden Gruppen werden große Unterschiede sichtbar. Professoren mit doppelter Staatsangehörigkeit haben sowohl unter Early Migrants (2,34 zu 1,81) als auch unter Student Migrants (2,63 zu 2,32) und Professional Migrants $(2,83 \mathrm{zu} 2,17)$ deutlich stärkere Beziehungen ins Herkunftsland als Professoren, die ausschließlich die deutsche Staatsangehörigkeit haben.

Die Interpretation dieser Ergebnisse hängt stark von den theoretischen Vorannahmen ab. Aus konservativer Sicht ließen sich die Zahlen als Beleg dafür anführen, dass eine doppelte Staatsangehörigkeit dazu führe, dass die Kontakte ins Herkunftsland stärker aufrechterhalten werden und somit die Gefahr einer geringeren Bindung und Identifikation mit Deutschland bestehe. Demgegenüber lässt sich allerdings anführen, dass der Index zur Interaktion mit Deutschen (siehe Abschnitt 6.8.1) sich zwischen Deutschen, Deutschen mit doppelter Staatsangehörigkeit und Ausländern kaum unterscheidet. Insofern lässt sich vielmehr festhalten, dass eine doppelte Staatsangehörigkeit die Chancen verbessert, vielfältige Kontakte und Interaktionen sowohl in Deutschland als auch im Herkunftsland aufzubauen.

Bemerkenswert sind auch die Unterschiede zwischen den drei „Staatsangehörigkeitstypen“ hinsichtlich der Meinung zum Herkunftsland. Knapp zwei Drittel (62\%) bewerten das Herkunftsland eher positiv, gut ein Drittel (34\%) neutral und $4 \%$ eher negativ. Dabei zeigt sich, dass Professoren mit doppelter Staatsangehörigkeit $(77 \%)$ ihr Herkunftsland am positivsten bewerten, vor Professoren 
mit ausländischer Staatsangehörigkeit (66 \%) und Professoren mit ausschließlich deutscher Staatsangehörigkeit (45\%). Die besonders kritische Perspektive auf das Herkunftsland von Menschen mit Migrationshintergrund, die die Staatsangehörigkeit des Herkunftslandes nicht besitzen, könnte unter anderem damit zusammenhängen, dass Personen aus dieser Gruppe möglicherweise zum Teil bewusst die Entscheidung getroffen haben, die Staatsangehörigkeit des jeweiligen Landes nicht zu behalten.

Interessant ist zudem die Frage, inwieweit zwischen den vier erstellten Clustern zur Interaktion in Deutschland und den Kontakten ins Herkunftsland auch ein direkter Zusammenhang bestehen kann. Hier zeigt sich, dass Professoren in Cluster 1 „Primär Deutschland“ $(1,72)$ mit Abstand die geringsten Aktivitäten ins Herkunftsland aufweisen, während Professoren in Cluster $2(2,49)$, Cluster 3 $(2,34)$ und Cluster $4(2,61)$ deutlich aktiver im Herkunftsland sind.

Abschließend sollen zwei unterschiedliche Erklärungsmodelle ${ }^{33}$ von Faist, Fauser und Reisenhauer (2014) zum Verhältnis von Transnationalität und Integration näher untersucht werden (vgl. Abschnitt 3.2.2.4). Der Ansatz „Linearer Transnationalismus" geht davon aus, dass Migranten nach der Zuwanderung soziale Kontakte in das Herkunftsland aufrechterhalten werden, diese aber mit zunehmender Aufenthaltsdauer und Integration in die Mehrheitsgesellschaft abnehmen. Eine Analyse der transnationalen Aktivitäten hinsichtlich der Aufenthaltsdauer in Deutschland zeigt, dass der Index von 2,64 für Migranten, die in den letzten 10 Jahren zugewandert sind, über 2,43 für Migranten, deren Zuwanderung zwischen 10 und 20 Jahren zurückliegt, über 2,26 bei Migranten, die bereits vor über 20 Jahren zugewandert sind, auf 2,12 bei Migranten der zweiten Generation kontinuierlich und signifikant abfällt. Die Ergebnisse deuten darauf hin, dass ein Zusammenhang zwischen Aufenthaltsdauer und transnationaler Aktivität besteht. Dennoch muss auch festgehalten werden, dass die Unterschiede nicht besonders groß ausfallen und dass transnationale Aktivität auch für Migranten, die bereits über 20 Jahre oder gar in der zweiten Generation hier leben, weiterhin eine bedeutende Rolle spielen.

Der Ansatz „Reaktiver Transnationalismus“ vertritt die These, dass Marginalisierungs- und Diskriminierungserfahrungen zur transnationalen Aktivität führen und es um die Suche nach Anerkennung und Prestige im Herkunftsland geht, die den Migranten im Zuwanderungsland verwehrt bleibt. Um diese These zu prüfen wird untersucht, inwieweit Professoren, die Nachteile aufgrund ihrer

\footnotetext{
${ }^{33}$ Der Ansatz des ressourcenabhängigen Transnationalismus (vgl. Abschnitt 3.2.2.3) konnte anhand der Befragung nicht analysiert werden. Die Vermutung liegt allerdings auch nahe, dass er für die Gruppe der Professoren mit Migrationshintergrund keine große Relevanz hat.
} 
Herkunft in Deutschland erlebt haben, in höherem Maße transnational aktiv sind. Insgesamt ein Fünftel der Professoren mit Migrationshintergrund gibt an, dass sie bereits aufgrund ihrer nationalen Herkunft Nachteile in Deutschland erfahren haben. Der Index der transnationalen Aktivität liegt für diese Gruppe bei 2,43 und damit nur geringfügig über dem Anteil von Professoren, die von keiner Benachteiligung sprechen 2,34. Ein Erklärungsansatz dafür, dass der reaktive Transnationalismus bei Professoren mit Migrationshintergrund kaum Erklärungskraft entfaltet, liegt vermutlich auch darin, dass Prestige und Anerkennung des Professorenberufs in Deutschland generell hoch ausfallen, so dass möglicherweise einzelne erlebte Benachteiligungen keine reaktive Reaktion nach sich ziehen.

\subsection{Vor- und Nachteile aufgrund von nationaler/ethnischer Herkunft, Geschlecht, Alter und Religion}

Eine typische Fragestellung bei Forschungen über Menschen mit Migrationshintergrund ist die Frage, inwieweit die Gruppe diskriminiert wird. Wie im Theorieteil (vgl. Abschnitt 3.2.3.2) ausgeführt, ist es wichtig, zwischen struktureller und subjektiver Diskriminierung zu differenzieren. Im Folgenden geht es ausschließlich um subjektive Diskriminierung. Wie im Theorieteil dargelegt, ist es wichtig, bei der Interpretation subjektiv empfundener Diskriminierung zu berücksichtigen, dass auch persönlicher Misserfolg, Unzufriedenheit oder Frustration dazu führen können, dass Diskriminierung als Argumentationsmuster genutzt wird. Generell zeigt die Forschungslage aber dennoch, dass subjektive Diskriminierung einen wichtigen Indikator für eine tatsächlich vorliegende Diskriminierung darstellt.

Häufig wird relativ schnell ein Zusammenhang zwischen bestimmten Negativerfahrungen und der Herkunft hergestellt. Dabei sollten allerdings zwei Aspekte berücksichtigt werden. Erstens kann es sein, dass bestimmte Benachteiligungen weniger auf die Herkunft als vielmehr auf andere Merkmale, wie beispielsweise das Geschlecht oder die soziale Herkunft, zurückzuführen sind. ${ }^{34}$ Zweitens kann die Herkunft zwar zu Benachteiligung und Diskriminierung führen, zugleich kann sie aber auch, beispielsweise im Kontext der Internationalisierung der Hochschulen, einen positiven Einfluss haben. Daher wird im Folgenden nicht nur nach

\footnotetext{
${ }^{34}$ Es geht dabei ausschließlich um die Frage, inwieweit subjektive Diskriminierungserfahrungen möglicherweise mit anderen leistungsunabhängigen Faktoren zu erklären sind. Rückschlüsse über strukturelle Diskriminierung, bspw. systematische Benachteiligungen bei der Anerkennung von Leistungen in Lehre und Forschung, sind nicht möglich.
} 
Negativerfahrungen aufgrund der Herkunft gefragt, sondern deutlich breiter untersucht, welche Vor- und Nachteile Professoren mit Migrationshintergrund aufgrund der nationalen Herkunft, des Geschlechts, des Alters, der ethnischen Herkunft und der Religion in Deutschland erfahren haben ${ }^{35}$.

Im ersten Schritt wird zunächst quantitativ betrachtet, wie häufig die einzelnen Merkmale genannt werden und welche demographischen und migrationsspezifischen Unterschiede bestehen. Anschließend werden im zweiten und dritten Teil dann die qualitativ inhaltsanalytisch ausgewerteten Erfahrungsbeispiele ${ }^{36}$ erst für die Vorteile und anschließend für die Nachteile anhand der Merkmale systematisch beschrieben und dargestellt. Abschließend geht es dann wiederum um eine quantitative Analyse zur Diskriminierung. Die Zustimmungswerte darüber, ob die Herkunft die wissenschaftliche Karriere positiv bzw. negativ beeinflusst habe, werden über zwei Likert Items abgebildet und hinsichtlich möglicher Gruppenunterschiede näher analysiert. Zudem wird untersucht, inwieweit Professoren mit Migrationshintergrund besonders häufig mit Themen betraut ${ }^{37}$ werden, die mit der internationalen Herkunft in Verbindung stehen und inwieweit sie sich mit Themen auseinandersetzen können, mit denen sie sich in ihrem Herkunftsland nicht beschäftigen könnten.

\subsubsection{Ausmaß der Vor- und Nachteile aufgrund verschiedener Merkmale}

Die Ergebnisse bestätigen die unterschiedlichen Wirkungen der genannten Merkmale. Während knapp ein Viertel der Professoren Nachteile aufgrund der nationalen Herkunft erlebt hat, sind es fast genauso viele, die auf Vorteile aufgrund der nationalen Herkunft verweisen (vgl. Abbildung 6.59). Insgesamt $8 \%$ der Befragten verweisen gleichzeitig auf Vor- und Nachteile.

In einem nächsten Schritt wurde betrachtet, ob sich Vor- und Nachteile aufgrund der nationalen Herkunft je nach Herkunftsland unterscheiden. Hier zeigt

\footnotetext{
${ }^{35}$ In Zukunft sollte auch der Aspekt der sozialen Herkunft berücksichtigt werden, da besonders aus der Bildungsforschung bekannt ist, dass das schlechtere Abschneiden von Schülern mit Migrationshintergrund häufig primär auf die soziale Herkunft zurückzuführen ist.

${ }^{36}$ Das methodische Vorgehen wird in Abschnitt 5.3 näher beschrieben.

${ }^{37}$ Die gewählte Formulierung im Rahmen der MOBIL-Studie, dass ,Professoren mit Themen betraut werden“", ist unglücklich gewählt. Es geht bei der Frage darum, inwieweit Professoren mit Migrationshintergrund aufgrund der Herkunft als besonders kompetent angesehen werden, bestimmte Funktionen zu übernehmen wie bspw. Gremientätigkeiten im Feld Diversity oder Internationalisierung. Ziel ist es, mögliche Zuschreibungsprozesse sichtbar zu machen.
} 


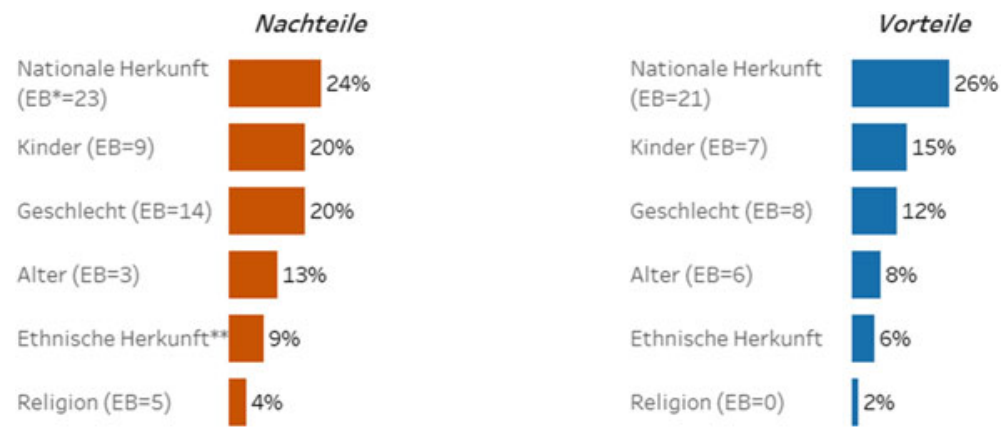

Abbildung 6.59 Vor- und Nachteile in Deutschland aufgrund unterschiedlicher Merkmale. (Quelle: MOBIL 2012/2013: *Anzahl der beschriebenen Erfahrungsbeispiele.**Erfahrungsbeispiele zur ethnischen Herkunft wurden aufgrund der zahlreichen Überschneidungen mit den Erfahrungsbeispielen der nationalen Herkunft zusammen mit diesen ausgewertet. Nationale Herkunft: Nachteile $\mathrm{N}=170$, Vorteile $\mathrm{N}=179$; Geschlecht: Nachteile $\mathrm{N}=167$, Nachteile $\mathrm{N}=175$; Kinder: Nachteile $\mathrm{N}=168$, Vorteile $\mathrm{N}=173$; Alter: Nachteile $\mathrm{N}=165$, Vorteile $\mathrm{N}$ = 171; Ethnische Herkunft: Nachteile $\mathrm{N}=166$, Vorteile 174; Religion: Nachteile $\mathrm{N}=166$, Vorteile $\mathrm{N}=172$ )

sich, dass Professoren aus Osteuropa (38\%) und aus Lateinamerika, Asien und Afrika (31\%) am häufigsten Nachteile aufgrund der Herkunft erlebt haben. Generell erleben Professoren aus Entwicklungs- und Schwellenländern häufiger Nachteile (45\%) als Professoren aus Industriestaaten. Bei den Vorteilen zeigen sich keine signifikanten Unterschiede bezüglich der Herkunftsländer. Allerdings zeigt sich im Rahmen der anschließend dargestellten Erfahrungsbeispiele, dass häufig Länder aus Europa, wozu speziell die skandinavischen Länder und das deutschsprachige Ausland zählen, in Deutschland ein besonders positives Image haben. Eine besondere Relevanz scheint die nationale Herkunft für Student Migrants zu haben. Von Professoren dieser Gruppe wird überdurchschnittlich häufig von Nachteilen (38\%), aber insbesondere auch von Vorteilen (44\%) berichtet. Professoren mit doppelter Staatsangehörigkeit verweisen ebenfalls überdurchschnittlich häufig auf Vorteile (34 \%), was möglicherweise genau darin begründet liegt, dass hier Menschen auch rechtlich Möglichkeiten multipler Identitäten ermöglicht werden und nicht vor die Wahl gestellt werden sich exklusiv auf eine Nationalität festzulegen. Demgegenüber berichten speziell Frauen (34 \%) und Professoren an Fachhochschulen (46\%) besonders häufig von Nachteilen.

Auch auf geschlechtsspezifische Vor- und Nachteile wird hingewiesen, wenngleich hier die Nachteile stärker hervortreten (vgl. Abbildung 6.59). Dabei ist 
auffällig, dass Frauen sowohl deutlich häufiger auf Vorteile (32\%) als auch auf Nachteile (47\%) verweisen. Die Ergebnisse zeigen sehr deutlich, dass die Benachteiligungsstrukturen von Frauen in der Wissenschaft auch subjektiv von Professorinnen mit Migrationshintergrund wahrgenommen werden und zugleich, dass das Geschlecht für Frauen im positiven wie im negativen Sinne eine deutlich größere Rolle spielt.

Auch Vor- und/oder Nachteile aufgrund von Kindern werden angeführt (vgl. Abbildung 6.59). Dabei werden, wie zu erwarten, diese Vor- und Nachteile ausschließlich von Personen mit Kindern genannt. Wenn ausschließlich diese Gruppe berücksichtigt wird, berichten $20 \%$ dieser Gruppe von Nachteilen und $15 \%$ von Vorteilen aufgrund von Kindern. Dabei zeigt sich, wie die Ergebnisse in Abschnitt 6.7.3 bereits vermuten lassen, dass Frauen deutlich häufiger Nachteile aufgrund von Kindern erleben (33\%) als Männer (15\%). Auch das Alter wird etwas häufiger als Nachteil denn als Vorteil wahrgenommen (vgl. Abbildung 6.59). Frauen betrachten ihr Alter deutlich häufiger als Nachteil (21\%) im Vergleich zu Männern (7 \%).

Bei der ethnischen Herkunft stellt sich zunächst die Frage, welche Definition hier zugrunde zu legen ist (vgl. ausführlich Abschnitt 3.1.2). Die in den Erfahrungsbeispielen beschriebenen Nachteile beziehen sich auf Aspekte wie die Benachteiligung aufgrund ,ausländisch klingender" Namen, aufgrund der Hautfarbe oder generell einer von der Mehrheitsgesellschaft abweichenden Physiognomie. Interessant sind dementsprechend die Gruppenunterschiede hinsichtlich der Nachteile aufgrund der ethnischen Herkunft, wenngleich diese aufgrund der geringen Fallzahlen nur unter Vorbehalt betrachtet werden sollten. Während insgesamt lediglich $8 \%$ der Professoren von Nachteilen berichten, verweisen Professoren aus Osteuropa (22\%) sowie aus Lateinamerika, Asien und Afrika $(25 \%)$ deutlich häufiger auf Nachteile. Offensichtlich sind auch die deutlich höheren Anteile für Professoren aus Schwellen- und Entwicklungsländern (36\%). Interessant ist hinsichtlich der Benachteiligung aufgrund der ethnischen Herkunft auch, dass hier ausschließlich deutsche Professoren mit Migrationshintergrund darauf hinweisen, was wiederum zeigt, wie wichtig die Verwendung einer breiten Definition des Migrationshintergrundes ist und nicht ausschließlich die Gruppe der Ausländer zu betrachten, um Diskriminierung und rassistische Zuschreibungen fundiert zu untersuchen (vgl. dazu ausführlich Abschnitt 3.1.3).

Interessant ist, dass die nationale Herkunft sowohl im positiven als auch im negativen Sinne für eine deutlich größere Zahl der Professoren mit Migrationshintergrund eine Rolle spielt als die ethnische Herkunft. Aus meiner Sicht bieten sich hier zwei mögliche Erklärungsmuster an. Erstens lässt sich aufgrund der 
Herkunftsländer der Professoren vermuten, dass die Zahl der sogenannten „visible minorities " vergleichsweise klein ausfällt. Ein solcher Erklärungsansatz würde ethnische Herkunft in erster Linie als phänotypische Abweichung von der Mehrheitsgesellschaft verstehen. Wie im Theorieteil ausgeführt (vgl. Abschnitt 3.1.2) ist allerdings die Definition, was unter Ethnizität verstanden wird, keineswegs eindeutig. Die Frage, ob Professoren auf Vor- oder Nachteile aufgrund der ethnischen Herkunft verweisen, kann stark variieren, je nachdem ob sie eher ein Verständnis der primordialist ethnicity, der situational ethnicity oder der instrumental ethnicity zugrunde legen (vgl. Abschnitt 3.1.2). Das heißt, der deutlich häufigere Verweis auf die nationale Herkunft im Vergleich zur ethnischen Herkunft könnte also auch darin begründet liegen, dass die Professoren eine deutlich klarere Vorstellung darüber haben, was mit dem Konzept der nationalen Herkunft gemeint ist im Vergleich zur ethnischen Herkunft.

$\mathrm{Zu}$ Beginn des Kapitels wurde der Aspekt der Diskriminierung angesprochen. Insgesamt deuten die Zahlen darauf hin, dass etwa ein Viertel der Professoren mit Migrationshintergrund Benachteiligungen aufgrund der Herkunft erlebt. Zugleich gibt es aber auch eine fast genauso große Gruppe, die in diesem Zusammenhang auf Vorteile hinweist. Diese Zahlen verdeutlichen die Relevanz einer differenzierten Betrachtung. Die Analysen machen deutlich, dass Diskriminierung für bestimmte Gruppen insbesondere Professoren aus Entwicklungs/Schwellenländern ein größeres Problem darstellt. Da diese Gruppen unter den Professoren mit Migrationshintergrund aber insgesamt unterrepräsentiert sind, fallen entsprechende Diskriminierungsstrukturen auf den ersten Blick nicht so stark auf. Hier wird deutlich, wie wichtig es wäre, über eine deutschlandweite Befragung statistisch sichere Erkenntnisse zu gewinnen, die darüber Aufschluss geben können, inwieweit Diskriminierung ein strukturelles Problem für bestimmte Gruppen innerhalb der Professoren mit Migrationshintergrund darstellt.

Im Anschluss an die Frage, ob sie aus einem oder mehreren der genannten Gründe bereits Vor- oder Nachteile erlebt haben, wurden die Professoren gebeten, Erfahrungsbeispiele sowohl für positive als auch für negative Erfahrungen zu schildern. Insgesamt haben 35 Professoren Vor- und 45 Professoren Nachteile ausführlich beschrieben. Die Gesamtzahl der Erfahrungsbeispiele liegt noch etwas höher, da einzelne Professoren zum Teil Ausführungen zu mehreren Dimensionen vorgenommen haben. Das inhaltsanalytische Verfahren orientiert sich dabei an dem methodischen Vorgehen von Kuckartz (2016) sowie an dem methodischen Ansatz von Züll und Menold (2014), die sich spezifisch mit der Auswertung offener Fragen im Rahmen standardisierter Befragungen auseinandersetzen.

Die Erfahrungsbeispiele können sich dementsprechend auf alle sechs genannten Gründe beziehen. Vor diesem Hintergrund wurde im ersten Schritt eine 
"Grobcodierung“ vorgenommen, auf welche Gründe sich das beschriebene Erfahrungsbeispiel bezieht, wobei sich einzelne Erfahrungsbeispiele, wie beschrieben, auch auf mehrere Dimensionen beziehen können. Zur Unterstützung der Zuordnung wird zudem betrachtet, welchen Vor- und Nachteilen bezüglich der sechs Dimensionen die Professoren bei der ersten quantitativen Zuordnung zugestimmt haben. Ansonsten orientiert sich diese der Inhaltsanalyse an der deduktiven bzw. A-priori-Kategorienbildung nach Kuckartz (2016).

\subsubsection{Erfahrungsbeispiele Vorteile}

Bei den Vorteilen bezieht sich der Großteil der Erfahrungsbeispiele $(n=21)$ auf die nationale Herkunft. Dabei beziehen sich einzelne Beispiele zugleich auch auf die ethnische Herkunft. Einzelne Beispiele werden auch bezüglich des Geschlechts $(n=8)$, des Alters $(n=6)$ und der Kinder $(n=7)$ geschildert, während zum Thema Religion keine Erfahrungsbeispiele vorliegen. Zudem finden sich drei Kommentare von Professoren, die darauf verweisen, dass sie in keinem der Bereiche Vorteile erfahren haben.

Die Erfahrungsbeispiele zu den Vorteilen der nationalen und ethnischen Herkunft werden anhand induktiver Kategorienbildung analysiert, um auf der Grundlage Argumentationsmuster herauszuarbeiten (Kuckartz 2016, S. 83-86). Dabei lassen sich drei Antwortmuster herausstellen: erstens Professoren, die auf das positive Image ihres Herkunftslandes in Deutschland verweisen $(n=5)$, zweitens Professoren, die darauf hinweisen, dass sie aufgrund ihrer europäischen Herkunft in gewisser Weise privilegiert sind $(n=3)$, und drittens Professoren, die auf spezifische Vorteile der nationalen und ethnischen Herkunft für Forschung und Karriere hinweisen $(n=6)$. In den weiteren Antworten $(n=7)$ werden Aspekte, wie das zweisprachige Aufwachsen der Kinder und der Wille der deutschen Gesellschaft zur Wiedergutmachung der NS-Zeit, thematisiert. Zwei Professoren verweisen darauf, dass Vorteile aufgrund der nationalen Herkunft erst bestehen, seitdem sie die deutsche Staatsangehörigkeit besitzen.

Im Folgenden wird für die ersten beiden Antwortmuster jeweils ein exemplarisches Zitat, ein so genanntes Ankerbeispiel, dargestellt, um die Muster stärker herauszuarbeiten. Bei der dritten Gruppe werden angesprochene Themenfelder kurz benannt.

Auf das positive Image ihres Herkunftslandes in Deutschland $(\mathrm{n}=5)$ verweisen Professoren aus Großbritannien, Österreich und den skandinavischen Ländern. Folgendes Zitat verdeutlicht die Erfahrungsbeispiele dieser Gruppe exemplarisch: 
„Deutsche finden Dänen im Allgemeinen nett, verlässlich, kooperativ, und werden selten enttäuscht. Allerdings gereicht einem dieses kooperative Verhalten regelmäßig selbst zum Nachteil" (Universitätsprofessor aus den Geistes- und Sozialwissenschaften).

Diese etwas ambivalente Beurteilung der sehr positiven Zuschreibungen in Bezug auf das Herkunftsland zeigt sich auch bei anderen Befragten.

Für die zweite Gruppe $(\mathrm{n}=3)$, die auf ihre privilegierte Stellung aufgrund der europäischen Herkunft hinweist, ist exemplarisch folgendes Zitat zu nennen:

„Weißen Männern europäischer Herkunft stehen weltweit alle Türen offen!“ (Universitätsprofessor in den Lebenswissenschaften aus Nordeuropa)

Gemeinsam ist allen drei Zitaten dieser Gruppe, dass darin aus einer weltweiten Ungleichheitsperspektive auf die eigene Herkunft geblickt und vor diesem Hintergrund das Privileg der europäischen Herkunft kritisch reflektiert wird.

In der dritten Gruppe werden unterschiedliche Vorteile der nationalen und ethnischen Herkunft für Forschung und Karriere betont. Vorteile als EnglishNative-Speaker, Vorteile im Hinblick auf international vergleichende Forschung oder auch die Möglichkeit, leichter internationale Kontakte und Netzwerke aufbauen zu können, werden in diesem Zusammenhang angeführt.

Vorteile aufgrund des Geschlechts werden primär von Frauen beschrieben (n =6). Lediglich zwei männliche Professoren geben an, dass sie vermuten, dass das männliche Geschlecht für den wissenschaftlichen Karriereverlauf von Vorteil sei. Professorinnen verweisen primär auf Frauenförderprogramme. Zudem wird davon berichtet, dass die Kombination, eine Professur innezuhaben und gleichzeitig Mutter zu sein, eine besondere Anerkennung findet. Eine Professorin aus den Ingenieurwissenschaften berichtet zudem von einer besonders großen Aufmerksamkeit für ihre Person aufgrund ihres Geschlechts, da es in ihrem Lehrund Forschungsbereich nur wenige Frauen gibt.

Vorteile aufgrund von Kindern werden fast ausschließlich von Männern geschildert. Dabei wird sowohl auf Vorteile bei der Anrechnung im Karriereverlauf verwiesen wie beispielsweise die Möglichkeit sich länger auf Juniorprofessuren zu bewerben oder auf staatliche Unterstützungen wie das Kindergeld. Ein Professor weist darüber hinaus auch auf Möglichkeiten der Integration in die deutsche Gesellschaft über neu entstehende Kontakte in Bildungsinstitutionen und Sportvereinen hin.

Ausschließlich Professoren aus der jüngeren Generation nennen Erfahrungsbeispiele hinsichtlich des Alters. Die Altersgrenze bei der Berufung auf eine 
Juniorprofessur, Vorteile bei der Projektförderung für ,gute junge Wissenschaftler" und bessere Bewerbungschancen für jüngere Wissenschaftler werden als Beispiele genannt. Zudem verweisen zwei Professoren darauf, dass es ein Vorteil war, dass sie im Vergleich zu den deutschen Kollegen zum Zeitpunkt der Promotion deutlich jünger waren.

Zudem gibt es auch drei Kommentare von Professoren, die auf keinerlei Vorteile hinweisen. Darin wird kritisiert, dass die Erfahrungen, die Professoren mit Migrationshintergrund an Hochschulen einbringen, von Kollegen und Hochschulleitungen nicht oder nur selektiv beachtet werden. Ebenfalls wird von Rassismus in Bewerbungsgesprächen, beim Kontakt mit Kollegen, bei Antragsstellungen und in Gremien berichtet. Derartige Kritikpunkte werden im Folgenden über negative Erfahrungsbeispiele bei der nationalen und ethnischen Herkunft noch ausführlicher und differenzierter betrachtet.

\subsubsection{Erfahrungsbeispiele der Nachteile}

Bei den Nachteilen aufgrund der nationalen und ethnischen Herkunft sollte zunächst noch einmal betont werden, dass über $70 \%$ der Professoren mit Migrationshintergrund davon sprechen, bisher in Deutschland keine Nachteile in diesem Kontext erfahren zu haben. Eine differenzierte Analyse der Nachteile zeigt dennoch, dass in den insgesamt über 20 Erfahrungsbeispielen unterschiedliche Formen von Rassismus zum Vorschein kommen. Insgesamt lassen sich dabei drei Antwortmuster identifizieren.

Erstens sind Erfahrungsbeispiele zu nennen, in denen unmittelbar eine minderwertige Behandlung oder Beurteilung durch Kollegen oder Vorgesetzte beschrieben wird $(\mathrm{n}=8)$. Die folgenden zwei Ankerbeispiele verdeutlichen die Erfahrungen dieser Gruppe exemplarisch:

"Man muss immer erst nachweisen, dass man nicht dümmer ist als der durchschnittliche deutsche Akademiker, bevor man akzeptiert wird" (Universitätsprofessor aus einem afrikanischen Land).

„Meine Herkunft hat leider für einige ehemalige Kollegen von mir eine Rolle gespielt. Sie konnten Ihre Vorurteile gegenüber Türken nicht überwinden " (Fachhochschulprofessor aus der Türkei).

Abfällige oder minderwertige Behandlungen stehen bei allen genannten Erfahrungsbeispielen im Vordergrund. Auch eine vermutete schlechtere Beurteilung 
von Studienleistungen, geringere Gehaltszulagen und unmittelbar diskriminierende Äußerungen aufgrund der Herkunft bei der Stellenbesetzung werden in diesem Zusammenhang angeführt.

Bei der zweiten Gruppe von Erfahrungsbeispielen handelt es sich eher um generelle Diskriminierungserfahrungen aufgrund der Herkunft, die sich nicht direkt auf die berufliche Situation beziehen $(n=5)$. Nachteile aufgrund eines „,ausländisch klingenden“ Namens oder aufgrund der dunkleren Hautfarbe werden hier ebenso angeführt wie die Konfrontation mit rassistischen Äußerungen und dem generellen Gefühl, nicht dazuzugehören.

Eine dritte Gruppe berichtet von Problemen mit der Aufenthaltsgenehmigung, bei Visaanträgen für Auslandsreisen und generell bei der Kommunikation mit Behörden $(n=6)$. Belastende Erfahrungen aufgrund befristeter Aufenthaltsgenehmigungen werden dabei ebenso hervorgehoben wie Probleme hinsichtlich der Verbeamtung und generell schwierige und intransparente Verwaltungsprozesse. Hinsichtlich der Aufenthaltsgenehmigungen lässt sich zumindest festhalten, dass alle Erfahrungsbeispiele von Professoren stammen, die bereits in den 1990erJahren oder früher nach Deutschland zugewandert sind. Inwieweit sich die Situation seitdem nicht nur für Professoren aus der Europäischen Union, sondern auch für Professoren aus Drittstaaten verbessert hat, sollte in zukünftigen Studien näher betrachtet werden.

Ein weiteres wichtiges Feld stellen Nachteile aufgrund des Geschlechts dar. Von den insgesamt 15 Erfahrungsbeispielen stammen lediglich drei von Männern, die sich allesamt auf Benachteiligungen aufgrund von Frauenquoten beziehen. Während ein Professor die Benachteiligung aufgrund von Frauenförderung sogar explizit begrüßt, kritisiert ein anderer Professor in diesem Zusammenhang die exklusive Betrachtung des Geschlechts, ohne dabei die familiäre Situation zu betrachten. Er kritisiert speziell, dass kinderlose Frauen bevorzugt wurden, während seine Auszeit zur Kinderbetreuung in keiner Form berücksichtigt worden sei. Alle weiteren zwölf Erfahrungsbeispiele stammen von Frauen. Dabei wird häufig eher allgemein auf die ,üblichen Probleme“ von Frauen verwiesen sowie auf das Phänomen der ,gläsernen Decke“38 . Zwei Professorinnen aus Skandinavien und Osteuropa beschreiben Deutschland auch im Vergleich zu ihren Herkunftsländern als sehr ,karrierefeindlich“ für Frauen.

Zudem gibt es drei Professorinnen, die die Verbindung der Benachteiligung von Geschlecht und Elternschaft explizit machen. Die Professorinnen betonen

\footnotetext{
${ }^{38}$ Gläserne Decke stellt eine Metapher dar für die Schwierigkeiten, mit denen Frauen beim Zugang zu Führungspositionen konfrontiert sind. Die Schwierigkeiten stehen häufig im Zusammenhang mit Stereotypen und Vorurteilen gegenüber Frauen, die allerdings schwer nachweisbar sind.
} 
eine strukturelle Benachteiligung von Frauen mit Kindern in der Wissenschaft unabhängig von der Herkunft und verweisen auf ein geringes Maß an Rücksichtnahme, insbesondere von Seiten der älteren männlichen Kollegen, sowie auf abgelehnte Anträge auf Teilzeitarbeit. Eine Professorin berichtet von der eigenen Stigmatisierung als „Rabenmutter“, als sie kurz nach der Schwangerschaft wieder in den Beruf zurückkehrte. Zugleich gibt es aber auch den Fall eines Vaters, der von massiver Benachteiligung aufgrund seines Geschlechts im Sorgerechtsstreit berichtet, die dabei auch kulturell legitimiert wurde (,,Bei uns gehören Kinder zur Mutter").

Zentrale Bedeutung kommt generell Problemen der Vereinbarkeit von Elternschaft und Karriere zu. Alle weiteren Erfahrungsbeispiele für die Benachteiligung aufgrund von Kindern berichten von Problemen bei der Kitaplatzsuche, bei der Betreuung von Kindern nach der Schule, bei der Vereinbarkeit von beruflichen Terminen und „Öffnungszeiten“ von Bildungseinrichtungen, von geringer Zeit für die Partnerschaft und von der geringen Berücksichtigung von Elternzeiten von Seiten der Berufungskommissionen.

Hinsichtlich der Benachteiligung aufgrund der Religion werden vier Erfahrungsbeispiele angeführt. Zwei Professoren berichten von Diskriminierungserfahrungen an bayerischen Hochschulen, zum einen hinsichtlich der Tätigkeit an einer kirchlichen Hochschule und zum anderen in Bezug auf Konkordats-Professuren in Bayern. Dabei handelt es sich um Lehrstühle, die nicht an theologischen Fakultäten angesiedelt sind, bei deren Besetzung die katholische Kirche allerdings ein Einspruchsrecht hat. Die anderen beiden Beispiele beziehen sich auf Diskriminierungen aufgrund der Herkunft aus einem mehrheitlich muslimischen Land.

Zwei Professoren thematisieren zudem ihre Homosexualität. Während der eine Professor sowohl von privaten als auch von beruflichen Nachteilen berichtet, gibt der zweite Professor an, bisher keine Nachteile aufgrund der sexuellen Orientierung erfahren zu haben.

Generell sind Fragen zur Konfession und zur sexuellen Orientierung aus datenschutzrechtlichen Gründen nur in Ausnahmefällen in Erhebungen zu finden. Hier besteht dementsprechend noch ein deutliches Desiderat, ob und in welcher Form beide Aspekte im beruflichen Alltag von Wissenschaftlern eine Rolle spielen. 


\subsubsection{Einfluss der Herkunft auf die wissenschaftliche Karriere und die berufliche Themenwahl}

Während es bisher um generelle Vor- und Nachteile in Deutschland aufgrund unterschiedlicher Merkmale ging, soll im Folgenden explizit der Einfluss der Herkunft auf die wissenschaftliche Karriere sowie auf mögliche Aspekte in Forschung und Lehre untersucht werden. ${ }^{39}$ Konkret wird untersucht, inwieweit Migranten aufgrund ihrer Herkunft eher Vor- oder Nachteile hinsichtlich ihrer wissenschaftlichen Karriere hatten. Zudem zeigen wissenschaftliche Studien, dass von Migranten, insbesondere bei Berufen im Bildungswesen, häufig erwartet wird, dass sie sich mit bestimmten Themen, die in Verbindung zu ihrer Herkunft stehen, auseinandersetzen. Diesbezüglich wird untersucht, ob sich dieser Befund auch für Professoren mit Migrationshintergrund zeigt. Darüber hinaus wird untersucht, inwiefern die Befragten auch Möglichkeiten sehen, sich mit Themen auseinanderzusetzen, mit denen sie sich im Herkunftsland nicht beschäftigen könnten. Bei allen Themenfeldern wird zugleich analysiert, inwieweit Gruppenunterschiede nach migrationsspezifischen, demographischen und beruflichen Merkmalen sichtbar werden.

Über die Hälfte der Professoren mit Migrationshintergrund berichtet, dass ihre Herkunft einen positiven Einfluss auf die Karriere hatte. Auffällig ist, dass bei über zwei Dritteln der Professoren, die in den letzten zehn Jahren nach Deutschland gekommen sind, von einem positiven Einfluss die Rede ist. Demgegenüber fallen die Unterschiede nach Migrationstypen nur gering aus, was darauf hindeutet, dass es möglicherweise weniger der Migrationsentschluss in einem bestimmten Alter bzw. in einer bestimmten Karrierephase ist, der sich als vorteilhaft erweist, sondern entsprechende Vorteile eher auf verstärkte Internationalisierungsbemühungen der Hochschulen seit Anfang der 2000er-Jahre zurückgehen. Da Internationalisierungsprozesse an Universitäten einen deutlich höheren Stellenwert einnehmen als an Fachhochschulen, könnte dieser Aspekt auch einen Erklärungsansatz dafür bieten, dass Universitätsprofessoren deutlich häufiger auf Vorteile verweisen als ihre Kollegen an Fachhochschulen. Auch hinsichtlich der Fächergruppen sind es insbesondere die am stärksten an Fachhochschulen vertretenen Fächer, die Ingenieurwissenschaften und die Sozial- und Verhaltenswissenschaften, in denen am seltensten von Vorteilen die Rede ist. Es

\footnotetext{
${ }^{39}$ Auch bei den Erfahrungsbeispielen zu den Vor- und Nachteilen wurden Bezüge zur wissenschaftlichen Karriere hergestellt. Die folgenden Ausführungen ermöglichen darüber hinaus aber Rückschlüsse und Gruppenunterschiede für alle befragten Professoren mit Migrationshintergrund.
} 
scheint demnach eine Kombination von Hochschulart, Fächergruppe und Zuwanderungszeitpunkt zu sein, die den Einfluss der Herkunft auf die wissenschaftliche Karriere bedingt (vgl. Abbildung 6.60).

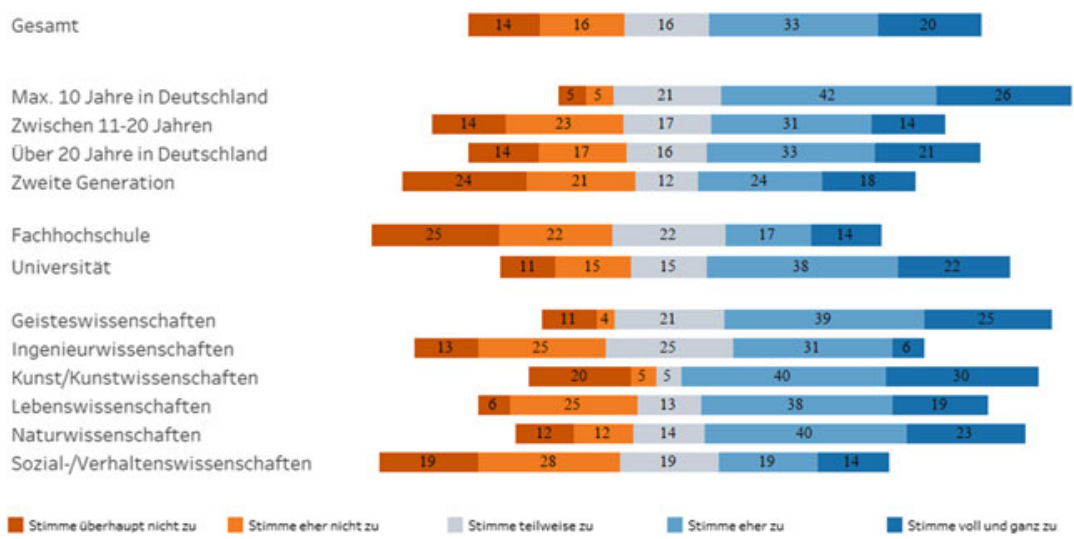

Abbildung 6.60 Positiver Einfluss des internationalen Hintergrundes auf die wissenschaftliche Karriere nach Dauer in Deutschland, Hochschulart und Fächergruppe. (Quelle: MOBIL 2012/2013 (Gesamt: $N=164(p=0,009)$, Dauer in Deutschland 2: $N=164(p=0,048)$, Hochschulart: $\mathrm{N}=164(\mathrm{p}=0,005)$ Fächergruppe $\mathrm{N}=159(\mathrm{p}=0,062))$; $\mathrm{p}$-value basiert auf Mann-Whitney-U-Test und Kruskal-Wallis-Test)

Von herkunftsbedingten Karrierehindernissen wird deutlich seltener berichtet. Lediglich knapp ein Drittel der Professoren spricht von entsprechenden Benachteiligungen. Während bei den Vorteilen keine herkunftsspezifischen Unterschiede sichtbar wurden, spielt der Aspekt bei Hindernissen eine deutlich größere Rolle. Über $40 \%$ der Professoren aus Entwicklungs- und Schwellenländern sprechen von Hindernissen, während nur circa ein Viertel der Professoren aus Industrieländern entsprechende Hindernisse im Rahmen der wissenschaftlichen Karriere wahrgenommen hat. Mit Abstand am seltensten berichten Professoren aus Österreich und der Schweiz von herkunftsbedingten Nachteilen für die eigene wissenschaftliche Karriere. Anhand der Paarvergleiche des Post-hoc-Tests zeigen sich signifikante Unterschiede zwischen Österreich und der Schweiz zu allen vier anderen regionalen Herkunftsregionen (vgl. Abbildung 6.61).

Eine Reihe von Studien gibt Hinweise darauf, dass speziell von Lehrern mit Migrationshintergrund erwartet wird, dass sie sich in besonderem Maße mit interkulturellen und internationalen Themen beschäftigen (vgl. Mantel und Leutwyler 


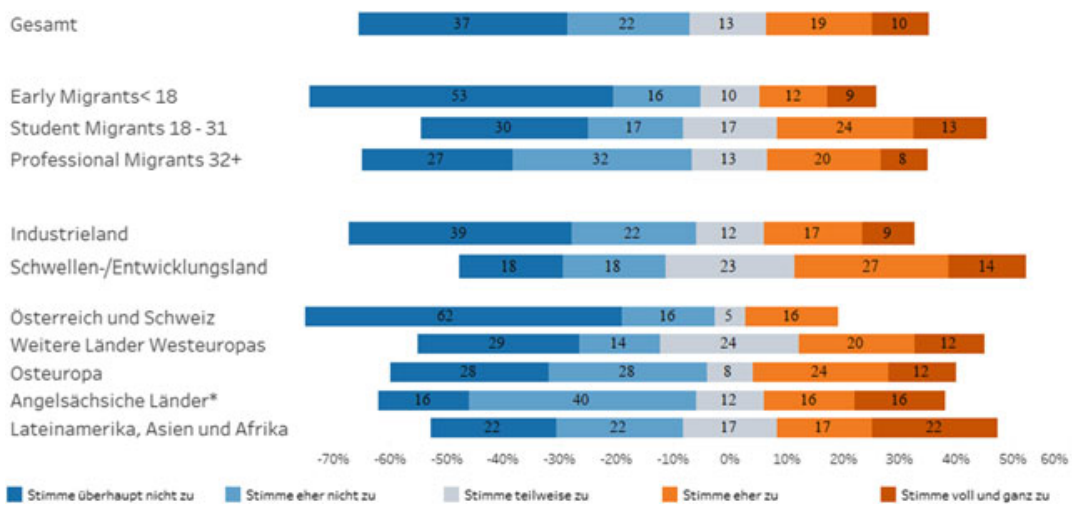

Abbildung 6.61 Karrierehindernisse aufgrund der internationalen Herkunft nach Herkunftsland und Migrationstypen. (*Kanada, USA und Australien. Quelle: MOBIL 2012/2013 (Gesamt: $\mathrm{N}=172$, Migrationstypen: $\mathrm{N}=174$ ( $\mathrm{p}=0,019)$, Entwicklungsstatus Herkunftsland: $\mathrm{N}=172(\mathrm{p}=0,036)$ Herkunftsregion $\mathrm{N}=172(\mathrm{p}<0,001))$; $\mathrm{p}$-value basiert auf Mann-Whitney-U-Test und Kruskal-Wallis-Test)

2013). Die Studie von Skachkova (2007) über Professorinnen mit Migrationsbiographie an Forschungsuniversitäten in den USA und die Untersuchung von Bakshi-Hamm mit wissenschaftlichen Mittelbauvertreterinnen in Deutschland berichten davon, dass Migrantinnen häufig der Status als Diversity-Experte zugeschrieben wird (vgl. Abschnitt 4.3.8).

Auch bei den Professoren mit Migrationshintergrund berichtet über ein Viertel, dass sie häufig mit Themen in Lehre und Forschung betraut werden, die mit ihrer internationalen Herkunft zusammenhängen. Dabei zeigen sich nur geringfügige Unterschiede zwischen Professorinnen und männlichen Professoren. Auffällig ist hingegen, dass die Erwartung speziell an Professoren aus Ländern außerhalb Europas herangetragen wird, von denen deutlich mehr als ein Drittel davon berichtet (vgl. Abbildung 6.62). Bei Professoren aus Entwicklungs- und Schwellenländern liegt der Anteil bei fast $40 \%$.

Eine Auseinandersetzung mit Themen, die im Herkunftsland nicht behandelt werden könnten, erfolgt bei knapp einem Fünftel der Befragten. Hier zeigen sich ähnliche Gruppenunterschiede wie bei der vorherigen Frage. Fast zwei Drittel der Professoren aus Entwicklungs- und Schwellenländern stimmen hier zu. Auch anhand der Paarvergleiche des Post-hoc-Tests zeigt sich, dass die Zustimmung von Professoren aus Lateinamerika, Asien und Afrika signifikant 
ch werde haufig mit Themen in Lehre und Forschung betraut, die mit meiner internationalen Herkunft zusammenhangen.

Gesamt

Osterreich und Schweiz

Weitere Lander Westeuropas

Osteuropa

Angelsachsische Lander*

Lateinamerika, Asien und Afrika
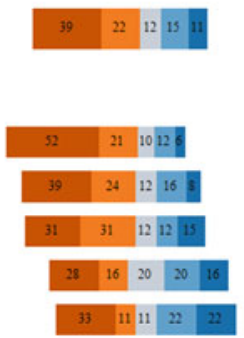

Dtimme teilweise zu ich kann mich mit Themen beschaftigen, mit denen ich mich in meinem Herkunftsland nicht auseinandersetzen könnte.
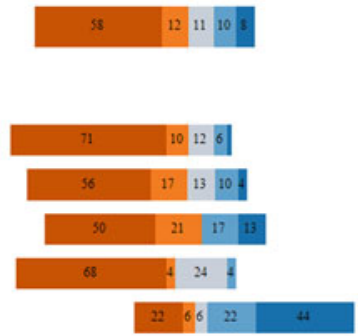

Stimme eher zu

Abbildung 6.62 Auseinandersetzung mit Themen nach Herkunftsregion. (*Kanada, USA und Australien. Quelle: MOBIL 2012/2013 Item 1: Gesamt: N = 170, Herkunftsregion: $N=$ 170 ( $\mathrm{p}=0,074)$, Item 2: Gesamt: $\mathrm{N}=166$, Herkunftsregion: $\mathrm{N}=166(\mathrm{p}<0,001)$ ); $\mathrm{p}$-value basiert auf Mann-Whitney-U-Test und Kruskal-Wallis-Test)

höher ausfällt im Vergleich zu allen vier anderen Herkunftsregionen (vgl. Abbildung 6.62). Hinsichtlich der Interpretation stellt sich die Frage, inwieweit die Zustimmung ausschließlich auf repressive politische Situationen in den Herkunftsländern zurückzuführen ist oder ob möglicherweise speziell im MINT-Bereich die fehlende Möglichkeit, sich mit bestimmten Themen auseinandersetzen zu können, auch in unzureichenden finanziellen Ressourcen und Sachmitteln begründet liegen kann. Im Fächervergleich zeigen sich keine signifikanten Unterschiede. Die Zustimmung liegt im Bereich „Humanities“ (19 \%) zwar etwas höher als im Bereich „Science“ (16\%), generell deuten die geringen Unterschiede aber darauf hin, dass sowohl politische Repression als auch fehlende Ausstattung der Beantwortung zugrunde liegen.

\subsection{Selbstverständnis der Professoren mit Migrationshintergrund}

Zum Abschluss der Befragung wurde allen Professoren mit Migrationshintergrund die offene Frage gestellt, inwieweit sie sich selbst als ,internationaler Professor" verstehen. Wie eingangs erläutert, wurde im Rahmen der MOBIL-Erhebung anstelle des Begriffs „Professor mit Migrationshintergrund“ die stärker positiv konnotierte Terminologie des ,,internationalen Professors“ verwendet. Dabei 
wurde den Befragten allerdings erläutert, dass unter ,internationalen Professoren“ alle Professoren zu fassen sind, die nach der Definition des Mikrozensus einen Migrationshintergrund ${ }^{40}$ besitzen. Bei dem Konzept des Migrationshintergrundes handelt es sich, wie bereits ausführlich dargelegt, um eine Fremdzuschreibung auf der Grundlage zuvor festgelegter Kriterien (vgl. Abschnitt 3.1). Die Frage, ob eine Person einen Migrationshintergrund aufweist, entscheidet sich zum Zeitpunkt der Geburt und dieser Status ist somit unveränderlich. Dementsprechend liegt das Ziel der abschließenden Fragestellung darin, zu untersuchen, inwieweit diese Fremdzuschreibung des ,internationalen Professors“ auch dem Selbstverständnis der Professoren entspricht und mit welcher Begründung sie das Konzept annehmen oder ablehnen. Eine erste Auswertung findet sich in dem Beitrag „Auf dem Weg zur Transnationalität? Eine explorative Studie über Professorinnen und Professoren mit Migrationsbiographie an deutschen Hochschulen" von Neusel und Wolter (2016). Dabei werden fünf typische Antwortmuster herausgearbeitet (Neusel und Wolter 2016, S. 51): Erstens „,die Selbstbeschreibung als internationale/r Wissenschaftler/in“, zweitens „, die Selbstbeschreibung primär als binational aufgrund der Herkunft“, drittens „Distanz gegenüber nationaler Zuordnung, Selbstwahrnehmung eher in transnationalen sozialen Räumen, auch wenn sie als international bezeichnet werden“, viertens „Hybride Identitäten“, fünftens „, Selbstbeschreibung als neue Deutsche".

Die fünf Antwortmuster werden jeweils anhand einer Reihe von Zitaten veranschaulicht. Diese explorativen Analysen bieten erste interessante Erkenntnisse über das Selbstverständnis und die Identität von Professoren mit Migrationshintergrund. Im Rahmen dieser Arbeit soll noch ein Schritt weiter gegangen werden, indem zu allen 134 Antworten eine umfassende inhaltsanalytische Auswertung durchgeführt wird. Das inhaltsanalytische Vorgehen orientiert sich - wie bei der Analyse zu den erlebten Vor- und Nachteilen in Deutschland (vgl. Abschnitt 6.9) an dem methodischen Ansatz von Kuckartz (2016) sowie Züll und Menold (2014). In einem ersten Schritt wird zunächst ,,deduktiv“ geprüft, ob die Professoren eher einem Selbstverständnis als ,internationaler Professor“ zustimmen oder ob sie sich eher nicht als solcher verstehen. Auf dieser Grundlage lassen sich in einem zweiten Schritt über induktive Analysen unterschiedliche Begründungsmuster herausarbeiten.

\footnotetext{
${ }^{40}$ Nach der Definition des Statistischen Bundesamtes haben Personen einen Migrationshintergrund, die selbst oder mindestens ein Elternteil nicht als Deutsche geboren wurden oder die selbst oder mindestens ein Elternteil nach 1949 nach Deutschland zugewandert sind und somit nicht auf dem heutigen Gebiet Deutschlands geboren wurden.
} 
Selbstverständnis als internationaler Professor?

- Ja/ eher ja

- Nein/eher nein

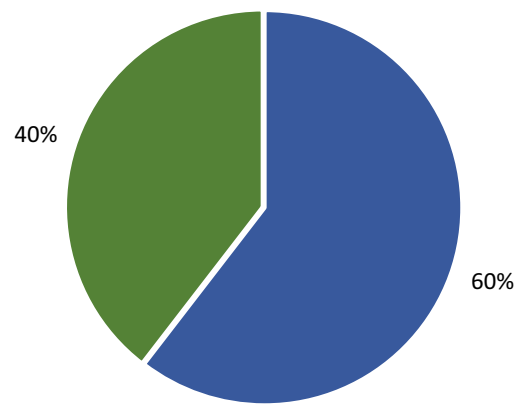

Begründung für Zustimmung

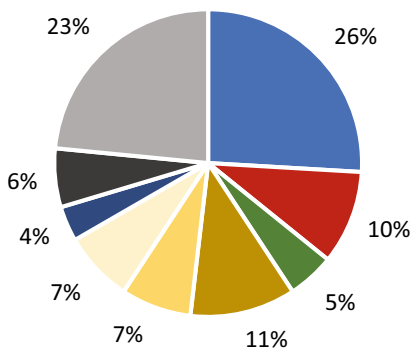

- Profession

- Biographie

- Profession und Biographie

- Herkunft

- Herkunft und Biographie

Herkunft und Profession

- Europäischer Professor

- Andere Begründung

- Ohne Begründung
Begründung für Ablehnung

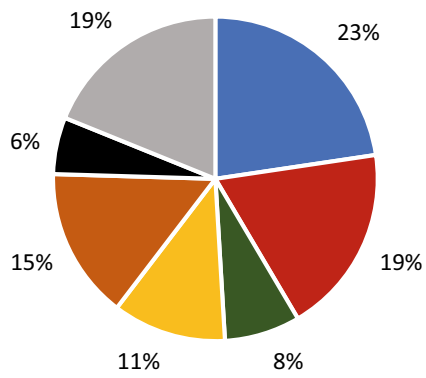

- Deutscher Professor

- Deutschsprachiges Ausland

- Mit Migrationshintergrund

- Professor aus Herkunftsland

- Ablehnung jeglicher Zuschreibung

- Nur bzgl. formaler Definition

- Weitere/Ohne Begründung

Abbildung 6.63 Positionierung und Begründung bzgl. des Selbstverständnisses als internationaler Professor. (Quelle: MOBIL 2012/2013: Selbstverständnis (Gesamt) N = 134; Begründung für Zustimmung $\mathrm{N}=81$; Begründung für Ablehnung $\mathrm{N}=53$ ) 
Im ersten Schritt zeigt sich zunächst, dass die meisten der befragten Professoren - insgesamt drei Fünftel - sich selbst als internationale Professoren verstehen (vgl. Abbildung 6.63).

Early Migrants verstehen sich ebenfalls zu über der Hälfte als internationale Professoren. Generell zeigen sich keine signifikanten Unterschiede nach Zuwanderungsalter, Herkunftsregion oder weiteren soziodemographischen Merkmalen. Lediglich bei der Staatsangehörigkeit werden deutliche Differenzen sichtbar. Unter Professoren, die ausschließlich die deutsche Staatsangehörigkeit haben, verstehen sich lediglich $39 \%$ als internationale Professoren. Interessant ist, dass Professoren, die sowohl die deutsche als auch eine ausländische Staatsangehörigkeit besitzen, sich mit Abstand am häufigsten als internationale Professoren verstehen (76\%) und damit noch deutlich häufiger als Professoren, die ausschließlich eine ausländische Staatsangehörigkeit vorweisen können (64\%).

\subsubsection{Argumentationsmuster für ein Selbstverständnis als internationaler Professor}

Hinsichtlich der Zustimmung zum Selbstverständnis als internationaler Professor lassen sich zentral drei Begründungsmuster für ein Selbstverständnis als identifizieren (vgl. Abbildung 6.63):

1. Begründungen in Bezug auf die Profession und die internationalen beruflichen Aktivitäten

2. Begründungen in Bezug auf die internationale Biographie

3. Begründungen in Bezug auf die internationale Herkunft

Zudem zeigen sich auch Kombinationen aus den drei Begründungsmustern. Eine kleine Gruppe von Professoren hebt ein Selbstverständnis als europäischer Professor hervor. Bei den ,,anderen Begründungen“ finden sich zum Teil auch kritische Begründungsmuster, wonach das eigene Selbstverständnis als internationaler Professor primär darin begründet liege, dass man selbst von der Gesellschaft nicht als Deutscher anerkannt werde.

Über ein Viertel und damit die größte Gruppe bilden Professoren, die ihr Selbstverständnis als internationaler Professor mit ihrer Profession begründen. Folgende zwei Beispiele spiegeln exemplarisch die entsprechenden Begründungsmuster wider: 
„Die Wissenschaft ist sehr international. Ich arbeite sehr international. Ich bin stolz und glücklich, zu dieser internationalen Gemeinschaft zu gehören “ (Universitätsprofessor, Ingenieurwissenschaften, aus Osteuropa).

„Versuche, meine Aufgaben in Forschung und Lehre mit einem Hauch aus niederländischem Flair zu verbinden. Das kommt offensichtlich gut an. Auch versuche ich im Rahmen der universitären Selbstverwaltung die Internationalisierung meiner Universität voran zu treiben “ (Universitätsprofessor aus den Naturwissenschaften).

Zudem wird das Selbstverständnis über Mehrsprachigkeit, mehrsprachige Lehre und Forschung sowie internationale Doktoranden und wissenschaftliche Kontakte begründet. Auch die Betreuung von internationalen Studierenden sowie Studierenden mit Migrationshintergrund spielt eine wichtige Rolle. Zwei Professoren begründen ihr Selbstverständnis als internationaler Professor explizit über ihre interkulturelle Kompetenz:

„Durchaus, ich habe ein erhöhtes Verständnis für Immigranten und Ausländer, bin mehr im Ausland als andere und kann leichter Erkenntnisse aus anderen Kulturkreisen in meine Arbeit integrieren" (Fachhochschulprofessor, Geistes- und Sozialwissenschaften, aus Nordeuropa).

„Insofern, als ich vielleicht öfter über "deutsche" Selbstverständlichkeiten nachdenke als manche deutschen KollegInnen und mich bemühe, Leuten ohne Ansehen ihrer Herkunft hier das Leben angenehm und nützlich zu machen (Studis, KollegInnen, Gästen) “ (Universitätsprofessorin, Geistes- und Sozialwissenschaften, aus Westeuropa).

Ein zweites wichtiges Begründungsmuster für ein internationales Selbstverständnis basiert auf der eigenen Biographie. Ein entsprechendes Begründungsmuster zieht jeder zehnte Professor heran. Dabei werden vor allem Studium und Berufserfahrungen hervorgehoben. Allerdings verweisen auch einige Professoren darauf, dass sie zwar eine internationale Biographie aufweisen, sich zugleich aber in Deutschland heimisch fühlen, wie das nachfolgende Zitat exemplarisch veranschaulicht:

„Da ich knapp die Hälfte meines Lebens im Ausland gelebt habe, trifft das zu. Da ich in Deutschland zur Schule gegangen bin und mein Erststudium in Deutschland gemacht habe, und immer schon die deutsche Nationalität habe, fühle ich mich nicht als Person mit Migrationshintergrund" (Fachhochschulprofessor, Ingenieurwissenschaften, aus Osteuropa).

Auch hier gibt es einen Professor, der explizit die interkulturelle Kompetenz und die Möglichkeit, aus einer Außenperspektive auf Deutschland blicken zu können, hervorhebt: 


\begin{abstract}
„Die meisten meiner Verwandten leben in England, viele leben in Deutschland und einige in den USA und in Südafrika. Der enge, langjährige und regelmäßige persönliche Kontakt mit meinen nichtdeutschen Verwandten erlaubt es mir, die Situation in Deutschland distanziert aber dennoch sehr wohlwollend zu sehen. Ich denke, die meisten Deutschen haben keine Ahnung, wie gut es ihnen eigentlich geht und welche Schwierigkeiten die Bürger vieler anderer Staaten haben. Ich denke, dass ich meine sehr tiefen und persönlichen Kenntnisse anderer Kulturen und Lebensläufe regelmäßig in meinen Alltag und in die Gespräche mit meinen Kollegen einbringe“ (Universitätsprofessor, Lebenswissenschaften, aus Nordeuropa).
\end{abstract}

Darüber hinaus gibt es eine kleine Gruppe, deren internationales Selbstverständnis sowohl über die Profession als auch über die Biographie hergestellt wird. Folgendes Zitat legt exemplarisch das Begründungsmuster dieser Gruppe dar:

„Die Internationalität ist für mich selbstverständlich, wenn ehemals auch eher paneuropäisch. Schule (hoher Ausländeranteil), Studium und Beruf habe ich immer in national gemischten Gemeinschaften verbracht. [Meine Wissenschaftsdisziplin] ist nur international überhaupt möglich“ (Fachhochschulprofessorin aus Westeuropa).

Professoren, die ihre Internationalität über die Profession begründen, betonen dabei häufig, dass das Verständnis als internationaler Professor sich exklusiv auf die Profession und z. T. auf die Biographie beziehe, dass die Herkunft damit aber in keinem Zusammenhang stehe, was sich anhand des folgenden kurzen Zitats prägnant verdeutlichen lässt:

„Ich bin international tätig - daher bin ich internationaler Professor, meine Herkunft hat damit nichts zu tun “ (Universitätsprofessorin, Geistes- und Sozialwissenschaften, aus Westeuropa).

Drei der vier Professoren, die eine Zuschreibung als internationaler Professor aufgrund der Herkunft ablehnen, gehören zur Gruppe der Early Migrants.

Während hier eine klare Distanzierung hinsichtlich der Zuschreibung einer internationalen Herkunft sichtbar wird, begründet über ein Viertel der Professoren das eigene Selbstverständnis als internationaler Professor explizit über die Herkunft, z. T. in Verbindung mit der Biographie oder Profession. In etwa jeder neunte Professor führt seine Internationalität exklusiv auf seine Herkunft zurück. Dabei betonen die meisten Professoren, dass sie zwar ihre Herkunft als international bezeichnen würden, dennoch aber ein starkes Zugehörigkeitsgefühl insbesondere zur Hochschule empfinden, was durch die folgenden beiden Beispiele veranschaulicht wird: 
„Von meiner Herkunft her bin ich einer, im Alltag spielt das aber keine Rolle. Ich bin an der Uni voll integriert, es spielt in unserem Kollegium oder in der Lehre keine Rolle, dass ich kein Deutscher bin “ (Universitätsprofessor, Geistes-/Sozialwissenschaften, aus Osteuropa).

„Ich verstehe mich als internationale Professorin. Ich fühle mich wohl an meiner Uni, aber spüre auch immer, sowohl positiv als negativ, dass ich eine andere Herkunft habe “ (Universitätsprofessorin, Geistes-/Sozialwissenschaften, aus Westeuropa).

Auch in dieser Gruppe gibt es einen Professor, der explizit die Verbindung herstellt zwischen der Herkunft und einer interkulturellen Kompetenz bzw. der Möglichkeit einer Außenperspektive, die er wie folgt darlegt:

„Trotz großer Nähe zwischen Deutschland und der Schweiz ist mir die nichtdeutsche Herkunft häufig bewusst bzw. für mich spürbar. Da ich dies als positiv empfinde - im Sinne einer produktiven Distanz - verstehe ich mich als international “ (Universitätsprofessor, Geistes-/Sozialwissenschaften).

Zudem beziehen sich jeweils $7 \%$ auf Herkunft und Biographie bzw. auf Herkunft und Profession. Bei der Gruppe, die anhand von Herkunft und Biographie argumentiert, gibt es sogar zwei Professoren, die im Ansatz sogar alle drei Aspekte ${ }^{41}$ einbeziehen, was sich anhand des folgenden Beispiels zeigen lässt:

„Aufgrund meiner Herkunft, meinem Aufwachsen, Leben, Studieren und Arbeiten
in verschiedenen Ländern in Asien, Europa und Nordamerika sehe ich mich eher
international als national verankert. Forschung und Lehre in meinem Fachgebiet der
[Wissenschaftsdisziplin] ist generell international, was besonders für meine Forschung
mit Kooperationen und Forschungsaufenthalten sowie Lehre in verschiedenen Ländern
aller Kontinente gilt“ (Universitätsprofessor, Naturwissenschaften, aus Westeuropa).

Beim Argumentationsmuster auf Grundlage von Herkunft und Profession wird eine Verbindung zwischen internationaler Herkunft mit internationalen Arbeitsgruppen, internationalen Aktivitäten im Ausland, internationalen Kooperationen, transkulturellen Themen und der Betreuung von Studierenden mit Migrationshintergrund hergestellt. Das folgende Beispiel gibt einen exemplarischen Einblick in die Begründungsmuster dieser Gruppe:

„Ja, ich bin eindeutig ein internationaler Professor, ich kann keine endgültigen Kriterien nennen, aber meine Herkunft (ich begann mit 23, Deutsch zu lernen, Schul-

\footnotetext{
${ }^{41} \mathrm{Da}$ es insgesamt nur zwei Fälle waren, wurde entschieden, keine gesonderte Gruppe für Herkunft, Biographie und Profession zu erstellen.
} 
und Studienabschluss aus meinem Herkunftsland) sowie mein thematisches Profil und meine Orientierung an internationalen Kongressen ergeben doch ein stimmiges Bild. Und dennoch bin ich ziemlich deutsch geworden" (Universitätsprofessor, Geistes-/Sozialwissenschaften, aus Lateinamerika).

Generell bleibt festzuhalten, dass die idealtypische Unterscheidung in Herkunft, Biographie und Profession an bestimmten Stellen Überschneidungen aufweist: erstens hinsichtlich Herkunft und Biographie, wenn der biographische Hintergrund zum Teil auch als Herkunft verstanden wird, und zweitens zwischen Biographie und Profession, wenn bestimmte internationale berufliche Aktivitäten sich sowohl auf aktuelle Tätigkeiten als auch auf länger zurückliegende Tätigkeiten beziehen.

Interessant ist zudem, dass einzelne Professoren ihre Internationalität über ein europäisches Selbstverständnis begründen. Positiv betrachtet, zeigt sich hier, dass die politischen Prozesse der europäischen Gemeinschaft der vergangenen Jahrzehnte sich auch im Selbstverständnis der Professoren widerspiegeln. Zugleich lässt sich aber auch einwenden, dass die Zahl der Professoren, die ein solches Selbstverständnis zugrunde legen, insbesondere wenn man bedenkt, dass fast drei Viertel der Professoren mit Migrationshintergrund aus dem europäischen Ausland kommen, doch vergleichsweise gering ausfällt.

\subsubsection{Argumentationsmuster gegen ein Selbstverständnis als internationaler Professor}

Insgesamt $40 \%$ der Professoren betonen demgegenüber, dass sie sich selbst nicht als internationaler Professor verstehen (vgl. Abbildung 6.63). Bei knapp einem Viertel und damit der größten Gruppe basiert die Begründung darauf, dass sie sich vielmehr als deutsche denn als internationale Professoren verstehen. Folgende zwei Antworten auf die Frage nach dem Selbstverständnis als internationaler Professor stehen exemplarisch für diese Gruppe:

„Gar nicht, da in Deutschland sozialisiert“ (Fachhochschulprofessor, Geistes- und Sozialwissenschaften, aus Asien).

„Eigentlich verstehe ich mich als deutscher Professor trotz meiner Geburt im Ausland, meiner ausländischen Frau und meines internationalen Werdegangs. Seltsam, wie schnell man in ihrer Definition international wird! " (Universitätsprofessor, Geistesund Sozialwissenschaften, aus Westeuropa). 
Weitere Professoren betonen für ihr Selbstverständnis als deutscher Professor das Aufwachsen in Deutschland sowie die heimische Verbundenheit zu diesem Land. Zugleich gibt es auch Professoren, die sich in ihrer Begründung stärker auf die Abgrenzung zum Herkunftsland oder den geringen Bezug zu zugewanderten Elternteilen beziehen. Insgesamt zehn Professoren - davon sieben aus Österreich und drei aus der Schweiz - begründen ihre Entscheidung damit, dass die Unterschiede zwischen Österreich/Schweiz und Deutschland sehr gering seien. Folgende zwei Zitate veranschaulichen die Begründungsmuster:

„Ich habe zwar nicht die deutsche Staatsangehörigkeit, aber alles andere an mir ist ziemlich deutsch. Ich habe mein Leben lang in Deutschland gelebt und Schweizerdeutsch als Muttersprache ist dem Hochdeutsch auch ziemlich nah " (Fachhochschulprofessorin, Naturwissenschaften).

„Die kulturelle Distanz zwischen Deutschland und Österreich ist nicht allzu groß. Es gibt zwar einige nicht zu verkennende Unterschiede, aber ich fühle mich eher nicht als internationale Professorin, allerdings als Professorin, die einiges an internationaler Erfahrung in Forschung und Lehre aufgrund der Tätigkeit an einer [auslandsprachigen] Business School in die Fakultät einbringt " (Universitätsprofessorin, Geistes- und Sozialwissenschaften).

Insbesondere das Begründungsmuster der zweiten Antwort, in der eine Zuschreibung als internationaler Professor abgelehnt, gleichzeitig aber auf internationale Aktivitäten verwiesen wird, findet sich bei vielen Professoren. Insgesamt vier Professoren, die alle zur Gruppe der Early Migrants gehören, betonen, dass sie sich nicht als internationaler Professor, sondern als Professor mit Migrationshintergrund bzw. als Professor der zweiten Generation verstehen.

Sechs Professoren lehnen das Selbstverständnis ab, da sie sich primär mit ihrem Herkunftsland identifizieren, wie durch die folgenden beiden Beispiele sichtbar wird:

„Ich bin Finnin und werde es in bestimmten Dingen immer bleiben “ (Universitätsprofessorin. Lebenswissenschaften).

„Als Niederländerin in Deutschland tätig“ (Universitätsprofessorin, Geistes- und Sozialwissenschaften).

Über $20 \%$ der nicht zustimmenden Antworten werden damit begründet, dass jegliche nationale Zuschreibung abgelehnt werde. Zudem betonen die Professoren in diesen Fällen, dass sie ausschließlich aufgrund der formalen Kriterien der Befragung als internationaler Professor klassifiziert worden seien, ihr Selbstverständnis 
damit aber in keiner Weise korrespondiere. Folgende drei Statements verbildlichen diese kritische Haltung:

„Ich sehe mich als Dozent, der den jungen Sängern das Singen beibringt. Egal, woher sie kommen "(Universitätsprofessor, Geistes- und Sozialwissenschaften).

„Ich denke international, ohne Personen Nationen zuzuordnen“ (Universitätsprofessorin, Naturwissenschaften).

„Ich denke ungerne in Kästchen. Ich bin ich“ (Universitätsprofessorin, Naturwissenschaften).

Zudem gibt es zwei Professoren, die ihre Ablehnung damit begründen, dass ihr Selbstverständnis weniger auf der Migrationsbiographie basiere, sondern primär darauf, dass sie aus sozial einfachen Verhältnissen kommen.

Zusammenfassend bleibt festzuhalten, dass die Profession und die Biographie eine grundlegende Bedeutung für ein Selbstverständnis als internationaler Professor haben. Demgegenüber gibt es zu der Frage, inwieweit die eigene Herkunft ein internationales Selbstverständnis begründet, unterschiedliche Standpunkte. Während es durchaus eine große Gruppe gibt, die einen unmittelbaren kausalen Zusammenhang herstellt, gibt es auch einzelne Professoren, die explizit darauf hinweisen, dass die Herkunft auf ihr internationales Selbstverständnis keinen Einfluss habe. Die kontroversen Standpunkte verdeutlichen, wie unterschiedlich die eigene Herkunft interpretiert werden kann. Es ist wichtig, diese Erkenntnis herauszustellen. Es lässt sich zwar aufgrund der Definition des Mikrozensus eine eindeutige Unterteilung in Professoren mit und ohne Migrationshintergrund vornehmen. In welcher Form Professoren mit Migrationshintergrund allerdings mit ihrer Herkunft umgehen und in welchem Maße sie ihre Herkunft mit ihrem eigenen Selbstverständnis in Verbindung bringen, liegt in erster Linie in den Händen der Professoren selbst und unterscheidet sich in hohem Maße.

Die Tatsache, dass es wichtig ist die Zuschreibung des Migrationshintergrundes nicht mit einem Selbstverständnis als Migrant gleichzusetzen, zeigt sich auch darin, dass es durchaus eine relevante Zahl von Professoren mit Migrationshintergrund gibt, die sich nicht als ,,internationaler Professor“, sondern als deutscher Professor verstehen. Die Tatsache, dass die Gruppe in erster Linie aus Early Migrants sowie aus Professoren aus Österreich und der Schweiz besteht, zeigt, dass Herkunftsland, Sprache und Aufenthaltsdauer dabei eine wichtige Rolle spielen. Die Heterogenität des Umgangs mit der Herkunft lässt sich darüber hinaus damit belegen, dass es auch eine Gruppe von Professoren gibt, die jegliche Zuschreibung aufgrund der Herkunft ablehnt. 
Zusammenfassend lässt sich festhalten, dass die Frage nach dem Selbstverständnis als internationaler Professor eine große Vielfalt unterschiedlicher Argumentationsmuster zum Vorschein bringt. Dies verdeutlich, wie unterschiedlich die eigene Migrationsbiographie und Internationalität verstanden und interpretiert werden kann. Zugleich ist allerdings auffällig, dass eindeutige Zuordnungen zu Nationalitäten und Ländern die Ausnahme darstellen, sondern dass vielmehr ein internationales Selbstverständnis aufgrund der Biographie und der Profession die Antwortmuster dominiert.

Open Access Dieses Kapitel wird unter der Creative Commons Namensnennung 4.0 International Lizenz (http://creativecommons.org/licenses/by/4.0/deed.de) veröffentlicht, welche die Nutzung, Vervielfältigung, Bearbeitung, Verbreitung und Wiedergabe in jeglichem Medium und Format erlaubt, sofern Sie den/die ursprünglichen Autor(en) und die Quelle ordnungsgemäß nennen, einen Link zur Creative Commons Lizenz beifügen und angeben, ob Änderungen vorgenommen wurden.

Die in diesem Kapitel enthaltenen Bilder und sonstiges Drittmaterial unterliegen ebenfalls der genannten Creative Commons Lizenz, sofern sich aus der Abbildungslegende nichts anderes ergibt. Sofern das betreffende Material nicht unter der genannten Creative Commons Lizenz steht und die betreffende Handlung nicht nach gesetzlichen Vorschriften erlaubt ist, ist für die oben aufgeführten Weiterverwendungen des Materials die Einwilligung des jeweiligen Rechteinhabers einzuholen. 